\title{
Palladium-Catalyzed Tandem Hydrocarbonylative Lactamization and Cycloaddition Reaction for Construction of Bridged Polycyclic Lactams
}

\author{
Pengcheng $\mathrm{Xu},{ }^{\mathrm{a}, \mathrm{c}}$ Bo Qian, ${ }^{* a}$ Bin $\mathrm{Hu}^{* \mathrm{a}}$ and Hanmin Huang*a,b \\ a State Key Laboratory for Oxo Synthesis and Selective Oxidation, Lanzhou Institute of \\ Chemical Physics, Chinese Academy of Sciences, Lanzhou 730000, P. R. China. \\ ${ }^{b}$ Hefei National Laboratory for Physical Sciences at the Microscale and Department of \\ Chemistry, Center for Excellence in Molecular Synthesis, University of Science and \\ Technology of China, Chinese Academy of Sciences, Hefei 230026, P. R. China. \\ 'University of Chinese Academy of Sciences, Beijing 100049, P. R. China. \\ *E-mail: $\underline{\text { boqian@licp.cas.cn, hcom@licp.cas.cn, hanmin@ustc.edu.cn }}$
}

\section{Contents}

1 General experimental details and materials........................................................S2

2 Preparation of the starting materials......................................................................S3

3 General procedure for the synthesis of products..........................................S6

4 Synthetic application for $1 \mathrm{mmol}-\mathrm{scale}$ reaction................................................S7

5 Preliminary mechanistic studies.........................................S8

6 Optimization of the reaction conditions.............................................................S11

7 Experimental characterization data for the starting materials......................S18

8 Experimental characterization data for the products......................................S24

9 X-ray crystal structure determination of the products.....................................S40

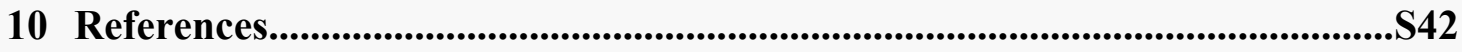

11 Copies of NMR for the staring materials and products................................S43 


\section{General experimental details and materials}

All non-aqueous reactions and manipulations were carried out with standard Schlenk techniques or in the glove box. All solvents before use were dried and degassed by standard methods and stored under nitrogen atmosphere. All reactions were monitored by TLC with silica gel-coated plates. NMR spectra were recorded on BRUKER AvanceIII (400 or $500 \mathrm{MHz}$ ) spectromete. Chemical shifts were reported in parts per million (ppm) down field from TMS with the solvent resonance as the internal standard. Coupling constants $(J)$ were reported in $\mathrm{Hz}$ and refered to apparent peak multiplications. High resolution mass spectra (HRMS) were recorded on Bruker MicroTOF-QII mass instrument (ESI). GC analysis were performed on Agilent 7890B with Hp-5 column. GC/MS were recorded on Agilent 7890A/5975C system. 


\section{Preparation of the starting materials}

Compounds $\mathbf{1 h}-\mathbf{1 i}$ were prepared according to the described procedure. ${ }^{1-3}$ Other starting materials (1a-1g, $\mathbf{1 m}, \mathbf{5 a - 5 f})$ were synthesized as following methods.

\subsection{Method A for the preparation of $1 \mathrm{a}-1 \mathrm{~g}, 1 \mathrm{~m}$}

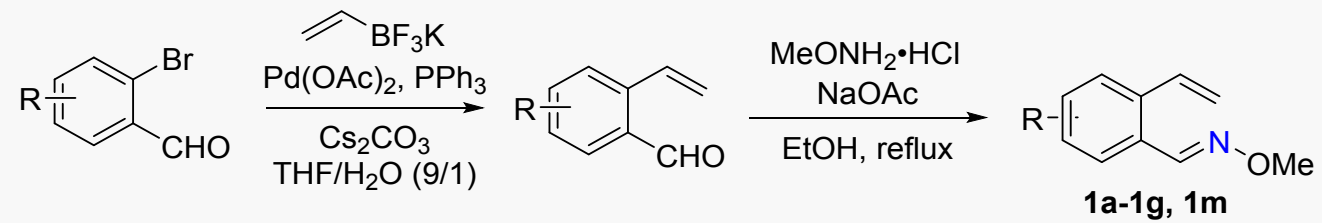

Step 1. To a solution of the substituted $o$-bromobenzaldehyde (10 mmol, 1 equiv) in $\mathrm{THF} / \mathrm{H}_{2} \mathrm{O}(9 / 1 \mathrm{v} / \mathrm{v}, 0.1 \mathrm{M})$, were successively added $\mathrm{Pd}(\mathrm{OAc})_{2}(0.5 \mathrm{mmol}, 0.05$ mol \%), $\mathrm{PPh}_{3}(1.0 \mathrm{mmol}, 0.1 \mathrm{~mol} \%)$, potassium vinyltrifluoroborate (12 mmol, 1.2 equiv) and $\mathrm{Cs}_{2} \mathrm{CO}_{3}$ (30 mmol, 3 equiv). After stirring for 5 minutes at room temperature in a sealed tube, the reaction temperature was elevated to $70{ }^{\circ} \mathrm{C}$ in an oil bath for 4 hours. The mixture was cooled to room temperature, in which $\mathrm{H}_{2} \mathrm{O}$ was added, following by extraction with ethyl acetate. The organic layers were combined, dried over $\mathrm{Na}_{2} \mathrm{SO}_{4}$, filtered and evaporated under reduced pressure. The crude product was purified by flash chromatography, employing mixtures of $n$-hexane/ethyl acetate as eluents to afford the aryl aldehyde compound. The spectra data matched those reported previously. ${ }^{2}$

Step 2. To a $50 \mathrm{~mL}$ round bottom flask with a stir bar was added aldehyde (5.0 mmol, 1 equiv), $\mathrm{MeONH}_{2} \cdot \mathrm{HCl}(7.5 \mathrm{mmol}, 1.5$ equiv), $\mathrm{NaOAc}$ ( $7.5 \mathrm{mmol}, 1.5$ equiv) and $\mathrm{EtOH}(20 \mathrm{~mL})$. The flask was heated under reflux in an oil bath for 2 hours. After cooling to room temperature, the mixture was extracted with EtOAc $(3 \times 10 \mathrm{~mL})$. The organic layers were combined, dried with $\mathrm{Na}_{2} \mathrm{SO}_{4}$, and concentrated to yield the crude product. The crude products was purified by column chromatography on silica gel to provide $1 \mathrm{a}-1 \mathrm{~g}, 1 \mathrm{~m} .{ }^{1}$ 


\subsection{Method B for the preparation of 5a-5f}

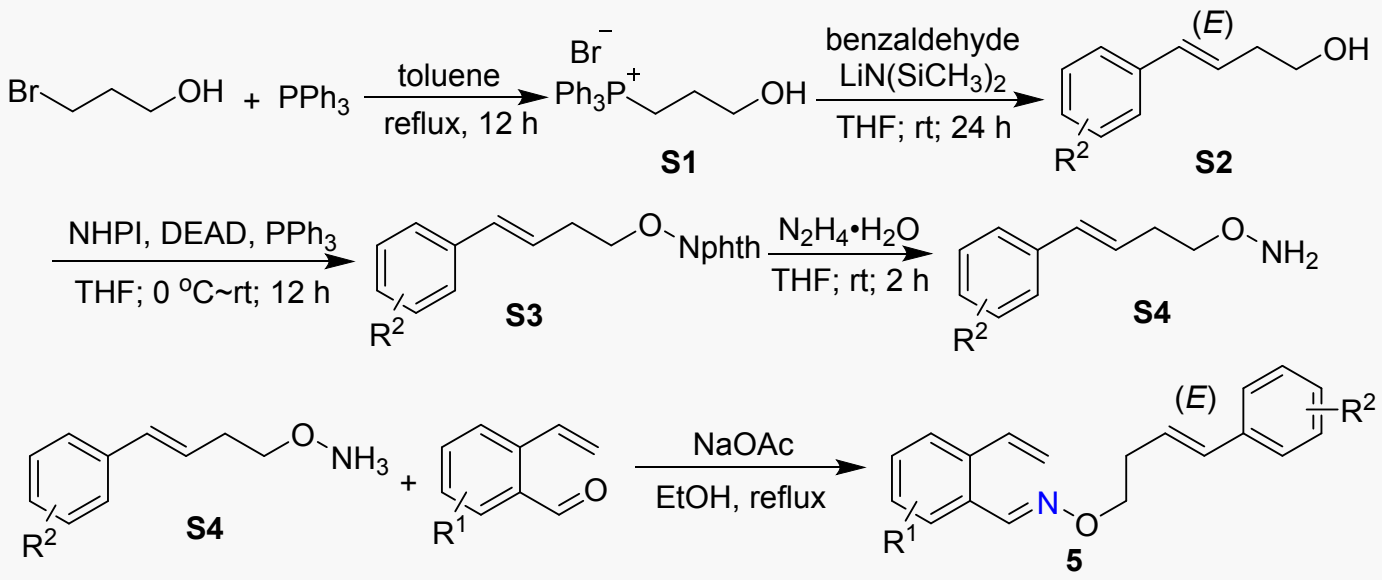

Step 1. A solution of 3-bromo-1-propanol $(2.3 \mathrm{~mL}, 25 \mathrm{mmol})$ and $\mathrm{PPh}_{3}(27.5$ mmol) in toluene $(10 \mathrm{~mL})$ were stirred under reflux in an oil bath for 16 hours. After cooling to room temperature, the reaction mixture was diluted with diethyl ether $(3 \times$ $50 \mathrm{~mL}$ ), and dried under vacuum to give the phosphonium salt $\mathbf{S 1}$ as a white solid. ${ }^{4}$

Step 2. A solution of lithium bis(trimethylsilyl)amide in tetrahydrofuran $(28 \mathrm{~mL}$, $1 \mathrm{~mol} / \mathrm{L}, 28 \mathrm{mmol}, 2.8$ equiv) was added dropwise at room temperature to a suspension of (3-propan-1-ol)triphenylphosphonium bromide $\mathbf{S 1}$ (12 mmol, 1.2 equiv) in dried tetrahydrofuran $(40 \mathrm{~mL})$. The solution was stirred for 0.5 hour, then $10 \mathrm{mmol}$ of benzaldehyde was added. The resulting reaction mixture was continuously stirred at room temperature. After 24 hours, saturated aqueous $\mathrm{NH}_{4} \mathrm{Cl}$ solution was added and the reaction mixture was then concentrated in vacuo. The residue was dissolved in dichloromethane, and washed with brine. The organic layer was separated, dried over $\mathrm{Na}_{2} \mathrm{SO}_{4}$, filtered and concentrated in vacuo. The residue was purified by chromatography on silica gel (10 40\% EtOAc in hexane) to give the desired product S2 ( $E$-isomer) ${ }^{4}$

Step 3: To a solution of the previously synthesized alcohol S2 (1 equiv) in THF (0.1 M) was added $\mathrm{PPh}_{3}$ (1.2 equiv) and $N$-hydroxyphthalimide (1.2 equiv). The resulting mixture was cooled to $0{ }^{\circ} \mathrm{C}$ before DEAD (1.2 equiv) was added dropwise in an ice bath. After 30 minutes at $0{ }^{\circ} \mathrm{C}$, the reaction medium was allowed to reach room temperature. Until the reaction was completed as monitored by TLC analysis, the 
solvent was removed under reduced pressure, and the residue was purified by flash chromatography on silica gel to afford the desired product $\mathbf{S 3} .^{5}$

Step 4: To a solution of the $N$-alkoxyphthalimides S3 (1 equiv) in DCM $(0.1 \mathrm{M})$ were added hydrazine hydrate (40\% in water, 6 equiv). After 2 hours, the mixture was filtered through Celite and concentrated in vacuo, giving the compound $\mathbf{S 4} .^{5}$

Step 5: To a $50 \mathrm{~mL}$ round bottom flask with a stir bar was added aldehyde (5 mmol, 1 equiv), oxyamine $\mathbf{S} 4$ (7.5 mmol, 1.5 equiv), $\mathrm{NaOAc}$ (7.5 mmol, 1.5 equiv) and $\mathrm{EtOH}(20 \mathrm{~mL})$. The reaction was stirred under reflux in an oil bath for 2 hours. After cooling to room temperature, the mixture was extracted with EtOAc $(3 \times 10$ $\mathrm{mL}$ ). The organic layers were combined, dried with $\mathrm{Na}_{2} \mathrm{SO}_{4}$, and concentrated to yield the crude product which was purified by column chromatography on silica gel to provide product 5a-5f. ${ }^{1}$ 


\section{General procedure for the synthesis of products}

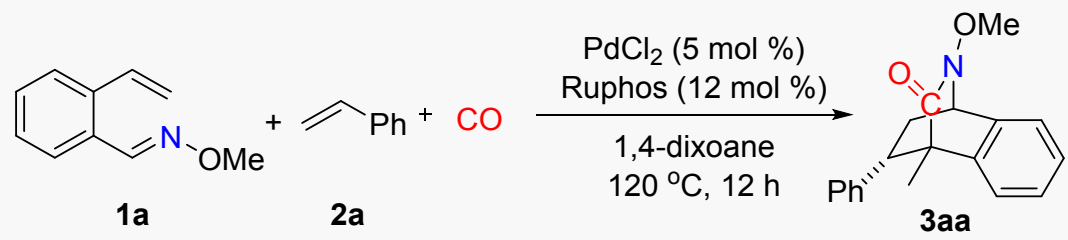

In the glove box, a mixture of $\mathrm{NH}_{4} \mathrm{Cl}(1.3 \mathrm{mg}, 0.025 \mathrm{mmol}, 5 \mathrm{~mol} \%), \mathrm{PdCl}_{2}(4.4$ mg, $0.025 \mathrm{mmol}, 5 \mathrm{~mol} \%)$, RuPhos (28 mg, $0.06 \mathrm{mmol}, 12 \mathrm{~mol} \%)$, alkene 1a (80.5 mg, 0.5 mmol, 1 equiv), styrene $2 \mathrm{a}$ (78 $\mathrm{mg}, 0.75 \mathrm{mmol}, 1.5$ equiv) and 1,4-dioxane (1.5 mL) were added into a dry glass vessel. The glass vessel was put into an autoclave and then taken out from glove box. The autoclave was purged and charged with $\mathrm{CO}(20 \mathrm{~atm})$. The reaction mixture was stirred at $120^{\circ} \mathrm{C}$ in a liquid alloy bath for 12 hours. After the reaction finished, the autoclave was cooled to room temperature, and the pressure was carefully released in the fume hood. The solvent was evaporated under reduced pressure and the resulting residue was purified by column chromatography on silica gel (petroleum ether/ethyl acetate $=75 / 25$ ) to afford the product 3aa. 


\section{Synthetic application for 1 mmol-scale reaction}

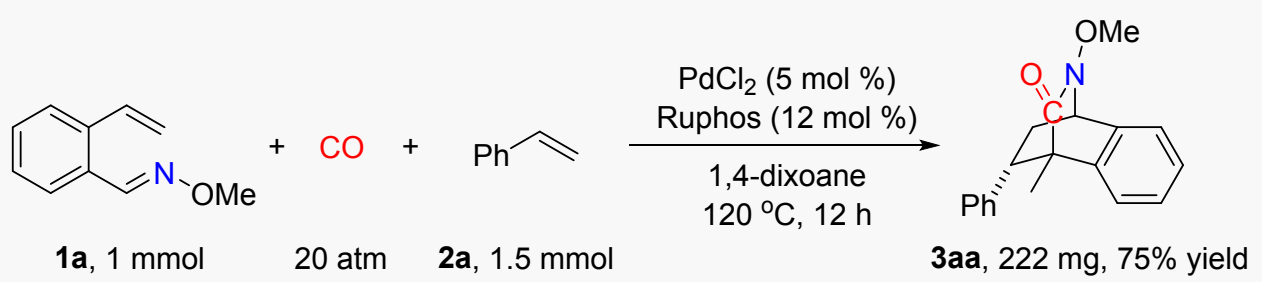

In the glove box, a mixture of $\mathrm{NH}_{4} \mathrm{Cl}(2.6 \mathrm{mg}, 0.05 \mathrm{mmol}, 5 \mathrm{~mol} \%), \mathrm{PdCl}_{2}(8.8$ mg, $0.05 \mathrm{mmol}, 5 \mathrm{~mol} \%)$, RuPhos (56 mg, $0.12 \mathrm{mmol}, 12 \mathrm{~mol} \%$ ), alkene 1a (161 mg, $1 \mathrm{mmol}, 1$ equiv), styrene $2 \mathrm{a}$ (156 mg, $1.5 \mathrm{mmol}, 1.5$ equiv) and 1,4-dioxane (3.0 $\mathrm{mL})$ were added into a dry glass vessel. The glass vessel was put into an autoclave and then taken out from glove box. The autoclave was purged and charged with $\mathrm{CO}$ (20 atm). The reaction mixture was stirred at $120{ }^{\circ} \mathrm{C}$ in a liquid alloy bath for 12 hours. After the reaction finished, the autoclave was cooled to room temperature, and the pressure was carefully released in the fume hood. The solvent was evaporated under reduced pressure and the resulting residue was purified by column chromatography on silica gel (petroleum ether/ethyl acetate $=75 / 25$ ) to afford the product 3aa in 75\% yield $(222 \mathrm{mg}$, endo/exo $=90: 10)$. 


\section{Preliminary mechanistic studies}

\subsection{The detection of intermediate VI}

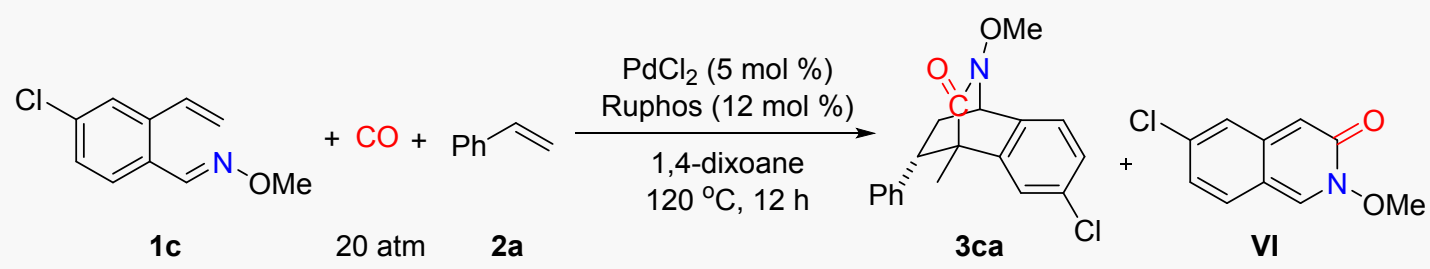

In the glove box, a mixture of $\mathrm{NH}_{4} \mathrm{Cl}(1.3 \mathrm{mg}, 0.025 \mathrm{mmol}, 5 \mathrm{~mol} \%), \mathrm{PdCl}_{2}(4.4$ mg, $0.025 \mathrm{mmol}, 5 \mathrm{~mol} \%)$, RuPhos (28 mg, $0.06 \mathrm{mmol}, 12 \mathrm{~mol} \%)$, alkene $1 \mathrm{c}$ (98.0 mg, $0.5 \mathrm{mmol}, 1$ equiv), styrene $\mathbf{2 a}$ (78 $\mathrm{mg}, 0.75 \mathrm{mmol}, 1.5$ equiv) and 1,4-dioxane (1.5 mL) were added into a dry glass vessel. The glass vessel was put into an autoclave and then taken out from glove box. The autoclave was purged and charged with $\mathrm{CO}(20 \mathrm{~atm})$. The reaction mixture was stirred at $120^{\circ} \mathrm{C}$ in a liquid alloy bath for 4 hours. After the reaction finished, the autoclave was cooled to room temperature, and the pressure was carefully released in the fume hood. The solvent was filtered by column chromatography on silica gel (acetate as the eluent). The liquid was then detected by GC/MS as following spectrum, and the molecular ion peak of $\mathbf{V I}$ has been found $(\mathrm{m} / \mathrm{z} 223)$. This result suggests that the intermediate VI might be involved in the transformation. 


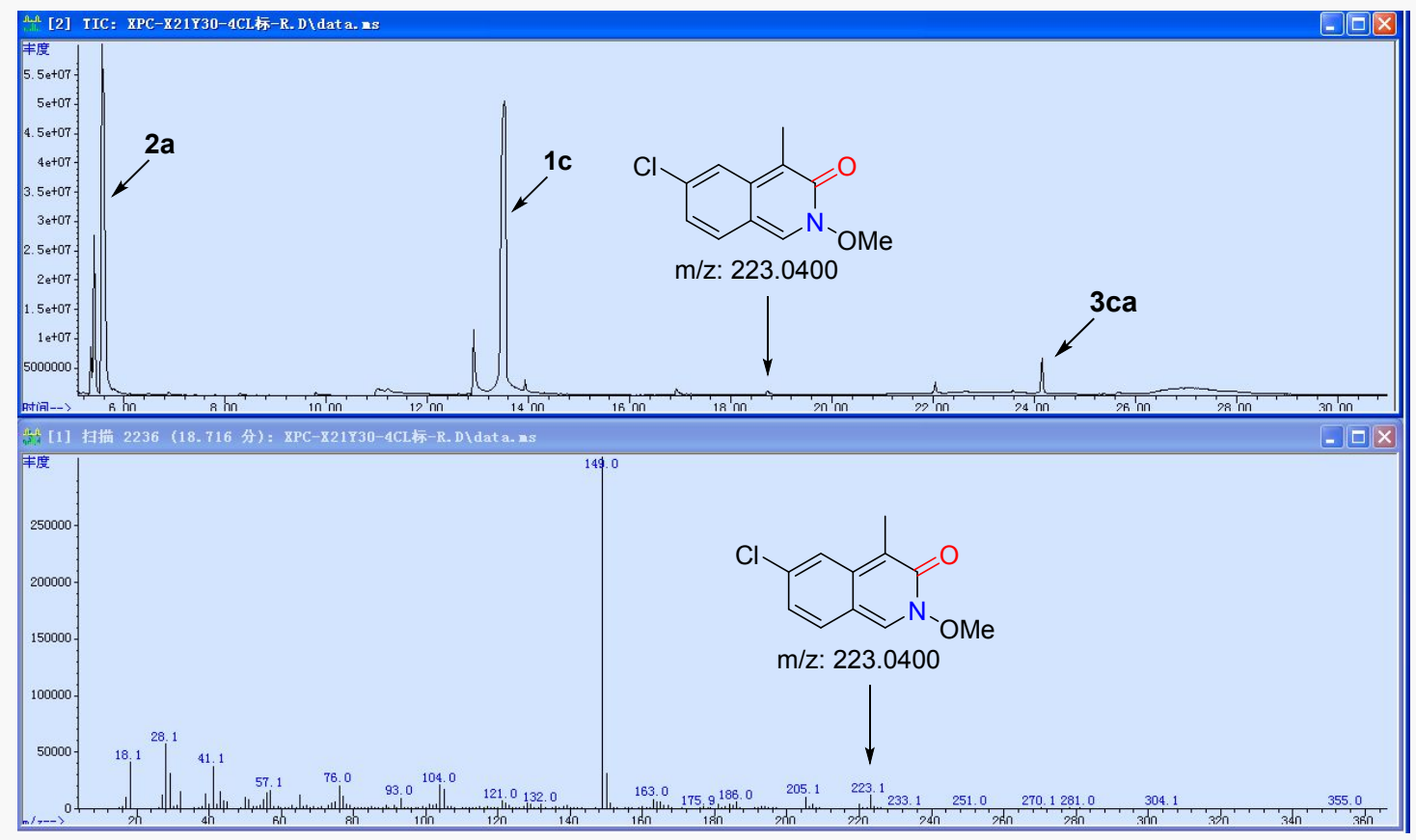

Figure S1. The detection of intermediate VI by GC/MS

\subsection{Experiments for the configuration inversion of isomers}

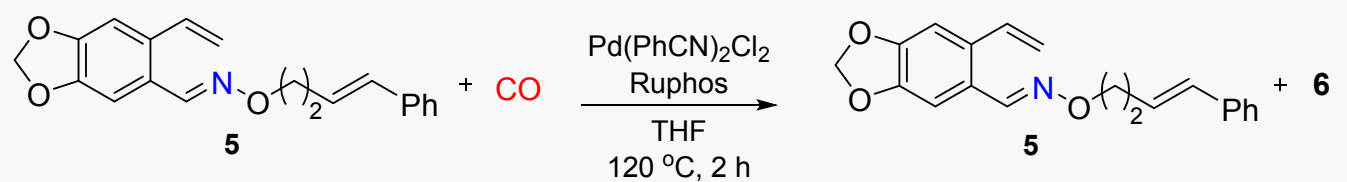

In the glove box, a mixture of $\mathrm{NH}_{4} \mathrm{Cl}(1.3 \mathrm{mg}, 0.025 \mathrm{mmol}, 5 \mathrm{~mol} \%$, $\mathrm{Pd}(\mathrm{PhCN})_{2} \mathrm{Cl}_{2}(9.6 \mathrm{mg}, 0.025 \mathrm{mmol}, 5 \mathrm{~mol} \%)$, RuPhos (28 mg, $0.06 \mathrm{mmol}, 12$ mol \%), alkene 5 ( $0.5 \mathrm{mmol}, 1$ equiv) and THF $(1.5 \mathrm{~mL})$ were added into a dry glass vessel. The glass vessel was put into an autoclave and then taken out from glove box. The autoclave was purged and charged with $\mathrm{CO}(20 \mathrm{~atm})$. The reaction mixture was stirred at $120^{\circ} \mathrm{C}$ in a liquid alloy bath for 2 hours. After the reaction finished, the autoclave was cooled to room temperature, and the pressure was carefully released in the fume hood. The solvent was evaporated under reduced pressure and the resulting residue was purified by column chromatography on silica gel (petroleum ether/ethyl acetate $=100 / 0 \sim 98 / 2)$ to afford the remained $\mathbf{5}$.

Furthermore, the configuration inverse of $\mathbf{5 c}$ and $\mathbf{5 d}$ could be obviously observed in the ${ }^{1} \mathrm{H}$ NMR spectra at $8.2 \sim 8.3 \mathrm{ppm}$. 

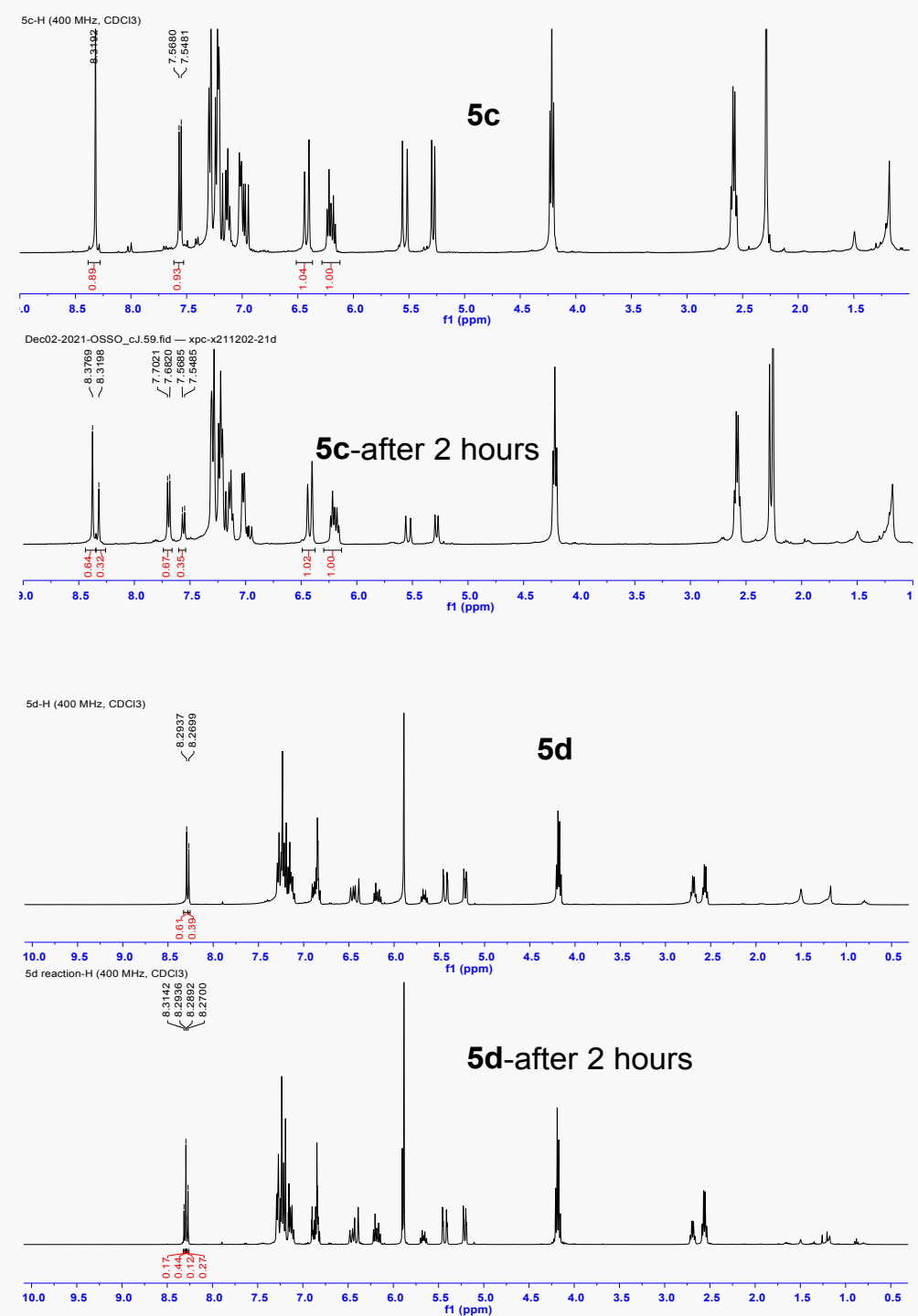

Figure S2. The detection of the configuration inverse of $\mathbf{5 c}$ and $\mathbf{5 d}$ by ${ }^{1} \mathrm{H}$ NMR 


\section{Optimization of the reaction conditions}

Table S1. Screening of catalysts for the synthesis of $3 \mathrm{aa}^{a}$

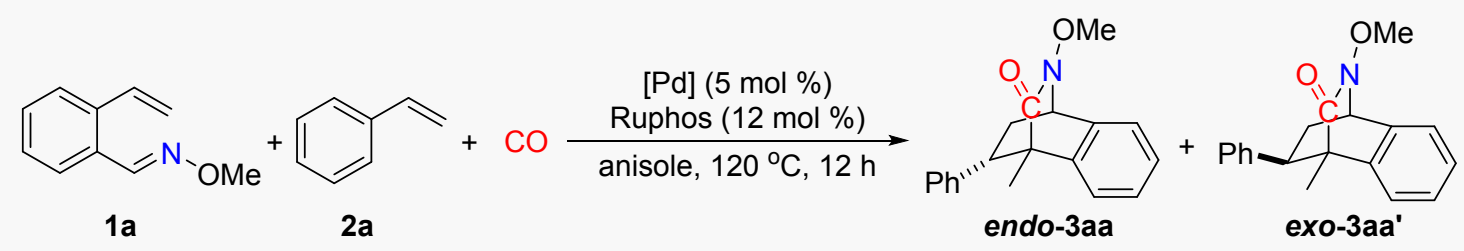

\begin{tabular}{|c|c|c|c|}
\hline Entry & Palladium catalyst & Yield $(\%)^{b}$ & endolexo ${ }^{b}$ \\
\hline 1 & $\mathrm{PdCl}_{2}$ & 59 & $90: 10$ \\
\hline 2 & $\mathrm{PdBr}_{2}$ & 0 & - \\
\hline 3 & $\mathrm{PdI}_{2}$ & 0 & - \\
\hline 4 & $\mathrm{PdCl}_{2}(\operatorname{cod})_{2}$ & 59 & $90: 10$ \\
\hline 5 & $\mathrm{Pd}(\mathrm{MeCN})_{2} \mathrm{Cl}_{2}$ & 50 & $90: 10$ \\
\hline 6 & $\mathrm{Pd}($ Xantphos $)\left(\mathrm{CH}_{3} \mathrm{CN}\right)_{2} \mathrm{OTf}$ & 0 & - \\
\hline 7 & $\operatorname{Pd}\left(t-\mathrm{Bu}_{3} \mathrm{P}\right)_{2}$ (without Ruphos) & 0 & - \\
\hline 8 & $\mathrm{Pd}(\mathrm{OAc})_{2}$ & 0 & - \\
\hline 9 & $\mathrm{Pd}_{2}(\mathrm{dba})_{3}$ & 8 & - \\
\hline 10 & $\mathrm{Pd}(\mathrm{PhCN})_{2} \mathrm{Cl}_{2}$ & 48 & $90: 10$ \\
\hline 11 & {$[\mathrm{Pd}(\text { allyl }) \mathrm{Cl}]_{2}$} & 57 & $90: 10$ \\
\hline 12 & $\mathrm{Pd}\left(\mathrm{PPh}_{3}\right)_{4}$ & 0 & - \\
\hline 13 & $\operatorname{Pd}(\mathrm{TFA})_{2}$ & 0 & - \\
\hline 14 & / & 0 & - \\
\hline
\end{tabular}

${ }^{a}$ Reaction conditions: 1a $(0.5 \mathrm{mmol}), 2 \mathrm{2a}(0.75 \mathrm{mmol})$, palladium catalyst $(5 \mathrm{~mol} \%)$, Ruphos (12 mol \%), $\mathrm{NH}_{4} \mathrm{Cl}(5 \mathrm{~mol} \%), \mathrm{CO}(20 \mathrm{~atm})$, anisole $(1.5 \mathrm{~mL}), 120{ }^{\circ} \mathrm{C}, 12$ hours. ${ }^{b}$ Yields and ratios of endo/exo were determined by $\mathrm{GC}$-analysis using $n$-tetradecane as an internal standard. 
Table S2. Screening of solvents for the synthesis of 3aa ${ }^{a}$

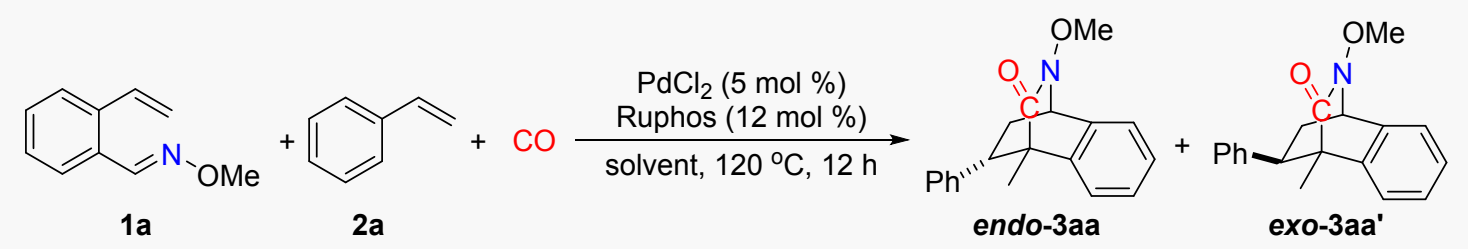

\begin{tabular}{cccc}
\hline Entry & Solvent & Yield $(\%)^{b}$ & endo/exo $^{b}$ \\
\hline 1 & anisole & 59 & $90: 10$ \\
2 & THF & 75 & $90: 10$ \\
3 & DMF & 43 & $89: 11$ \\
4 & DCM & Trace & - \\
5 & toluene & Trace & - \\
6 & NMP & 35 & $89: 11$ \\
7 & $1,4-d i x o a n e$ & $85(83)$ & $90: 10$ \\
8 & MeCN & 48 & $89: 11$ \\
9 & TBME & Trace & - \\
\hline${ }^{a}$ Reaction conditions: $\mathbf{1 a}(0.5 \mathrm{mmol}), \mathbf{2 a}(0.75 \mathrm{mmol}), \mathrm{PdCl}_{2}(5$ mol \%), Ruphos $(12$ mol \%), \\
NH ${ }_{4} \mathrm{Cl}$ (5 mol \%), CO $\left(20\right.$ atm), solvent $(1.5 \mathrm{~mL}), 120{ }^{\circ} \mathrm{C}, 12$ hours. ${ }^{b}$ Yields and ratios of \\
endo/exo were determined by GC-analysis using & $n$-tetradecane as an internal standard. \\
${ }^{c}$ Isolated yields. &
\end{tabular}


Table S3. Screening of ligands for the synthesis of 3aa ${ }^{a}$

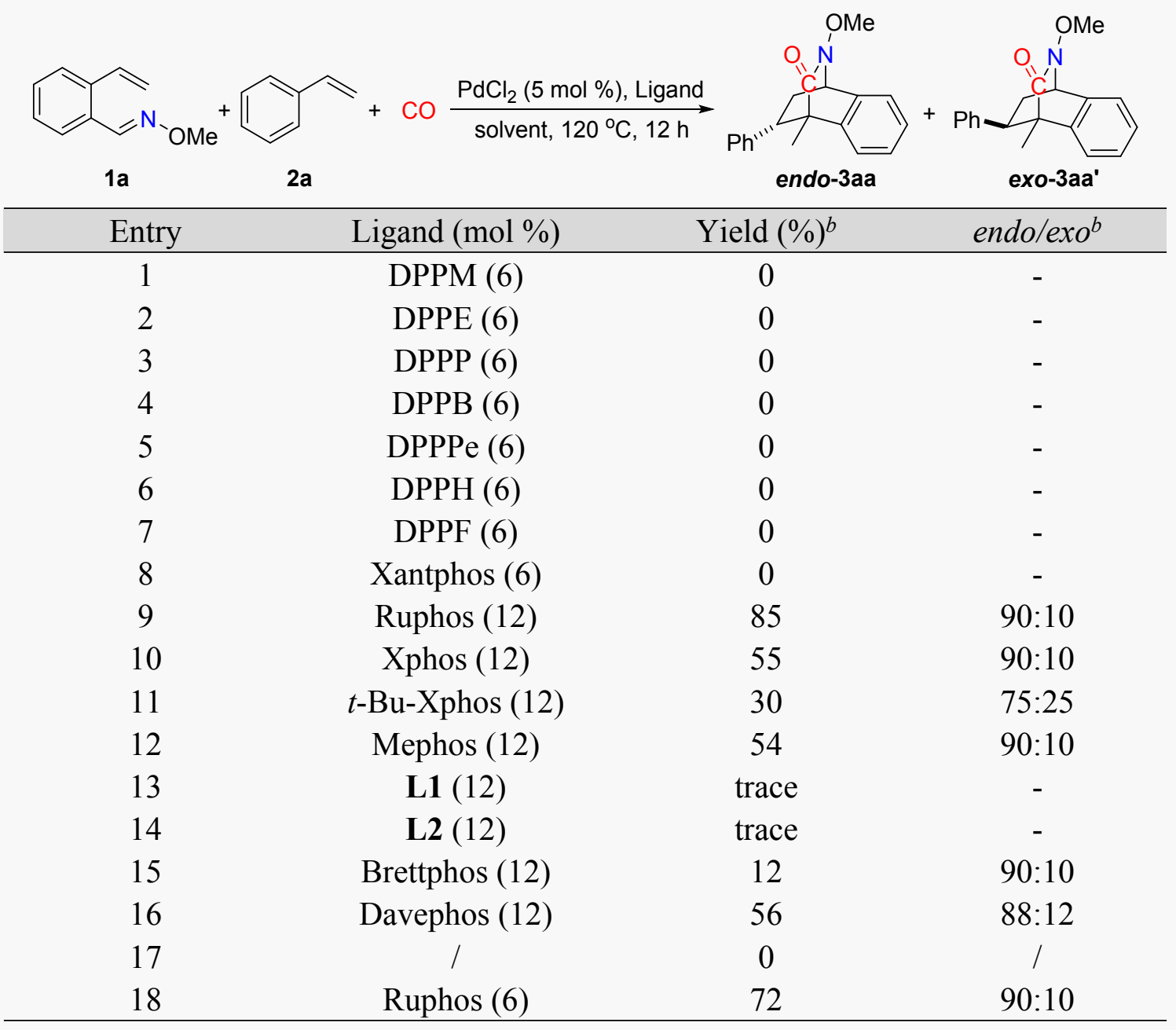

${ }^{a}$ Reaction conditions: 1a $(0.5 \mathrm{mmol}), 2 \mathrm{a}(0.75 \mathrm{mmol}), \mathrm{PdCl}_{2}(5 \mathrm{~mol} \%)$, Ligand (6 or 12 mol \%), $\mathrm{NH}_{4} \mathrm{Cl}(5 \mathrm{~mol} \%), \mathrm{CO}(20 \mathrm{~atm}), 1,4$-dioxane $(1.5 \mathrm{~mL}), 120^{\circ} \mathrm{C}, 12$ hours. ${ }^{b}$ Yields and ratios of endo/exo were determined by $\mathrm{GC}$-analysis using $n$-tetradecane as an internal standard.

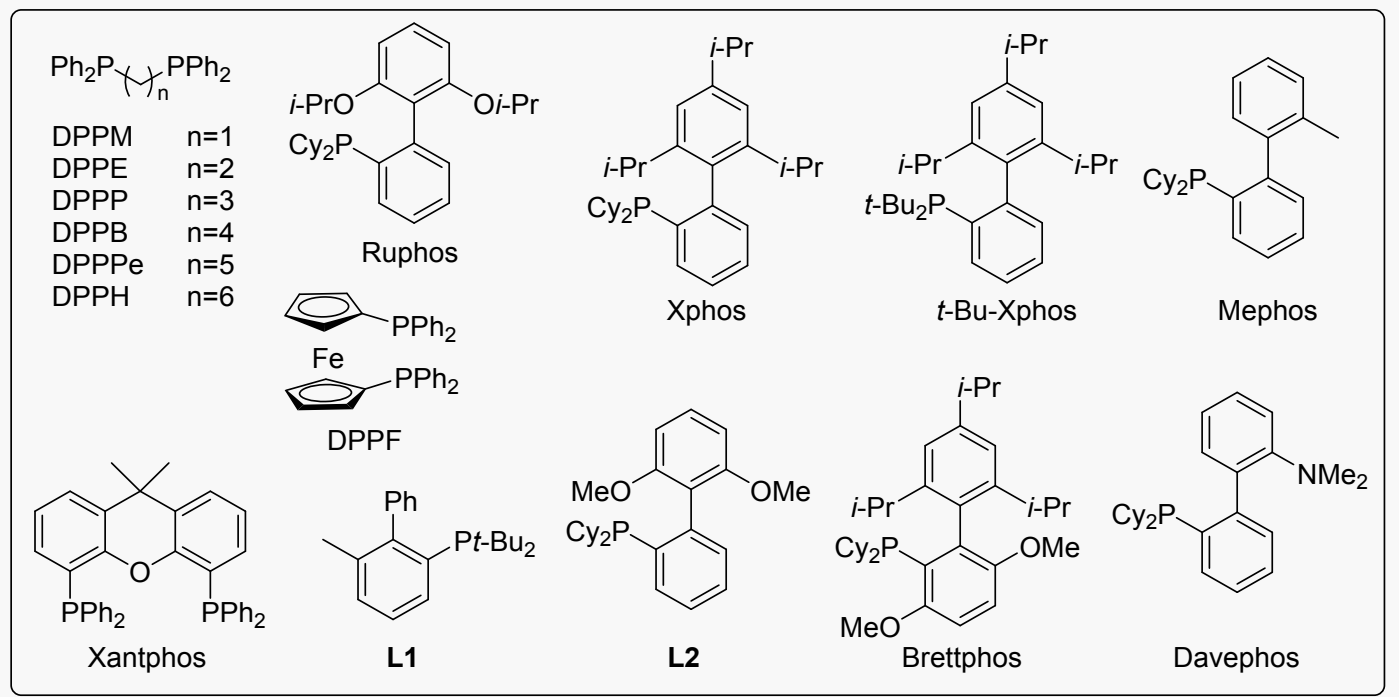


Table S4. Screening of CO pressure for the synthesis of $3 \mathrm{aa}^{a}$

\begin{tabular}{cccc} 
1a & & Yield $(\%)^{b}$ & endo/exo \\
\hline Entry & $\mathrm{CO}(\mathrm{atm})$ & trace & $90: 10$ \\
\hline 1 & 5 & 61 & $90: 10$ \\
3 & 10 & 85 & $90: 10$ \\
4 & 20 & 85 & $90: 10$ \\
\hline
\end{tabular}

${ }^{a}$ Reaction conditions: 1a $(0.5 \mathrm{mmol}), 2 \mathrm{a}(0.75 \mathrm{mmol}), \mathrm{PdCl}_{2}(5 \mathrm{~mol} \%)$, Ruphos (12 mol \%), $\mathrm{NH}_{4} \mathrm{Cl}(5 \mathrm{~mol} \%), \mathrm{CO}, 1,4$-dioxane $(1.5 \mathrm{~mL}), 120{ }^{\circ} \mathrm{C}, 12$ hours. ${ }^{b}$ Yields and ratios of endo/exo were determined by GC-analysis using $n$-tetradecane as an internal standard.

Table S5. Screening of additives for the synthesis of $3 \mathrm{aa}^{a}$

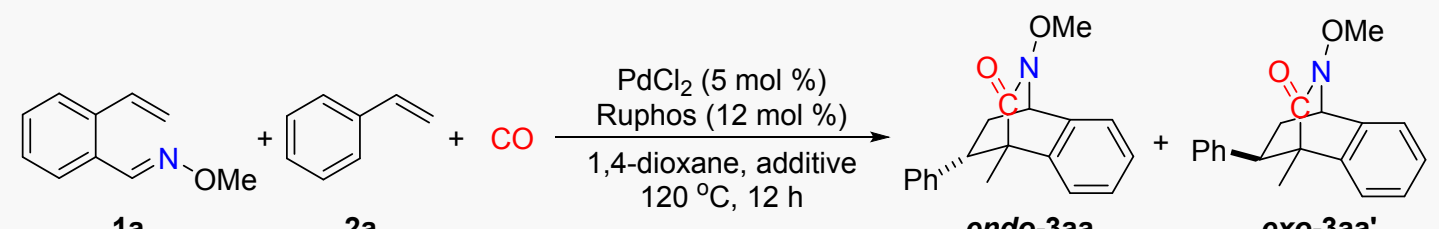

$1 \mathrm{a}$

$2 a$

endo-3aa

exo-3aa'

\begin{tabular}{cccc}
\hline Entry & Additive $(\mathrm{mol} \%)$ & ${\text { Yield }(\%)^{b}}^{b}$ & endo/exo $^{b}$ \\
\hline 1 & $\mathrm{NH}_{2} \mathrm{OH} \cdot \mathrm{HCl}(5)$ & 64 & $90: 10$ \\
2 & $\mathrm{NH}_{4} \mathrm{Cl}(5)$ & $85(83)^{c}$ & $90: 10$ \\
3 & $\mathrm{NH}_{4} \mathrm{Cl}(20)$ & 85 & $90: 10$ \\
4 & $\mathrm{NH}_{4} \mathrm{Cl}(50)$ & 85 & $90: 10$ \\
5 & $/$ & 63 & $90: 10$ \\
\hline
\end{tabular}

${ }^{a}$ Reaction conditions: 1a $(0.5 \mathrm{mmol}), 2 \mathrm{a}(0.75 \mathrm{mmol}), \mathrm{PdCl}_{2}(5 \mathrm{~mol} \%)$, Ruphos (12 mol \%), additive, $\mathrm{CO}(20 \mathrm{~atm}), 1,4$-dioxane $(1.5 \mathrm{~mL}), 120{ }^{\circ} \mathrm{C}, 12$ hours. ${ }^{b}$ Yields and ratios of endo/exo were determined by GC-analysis using $n$-tetradecane as an internal standard. 'Isolated yield. 
Table S6. Screening of catalysts for the synthesis of $6 a^{a}$

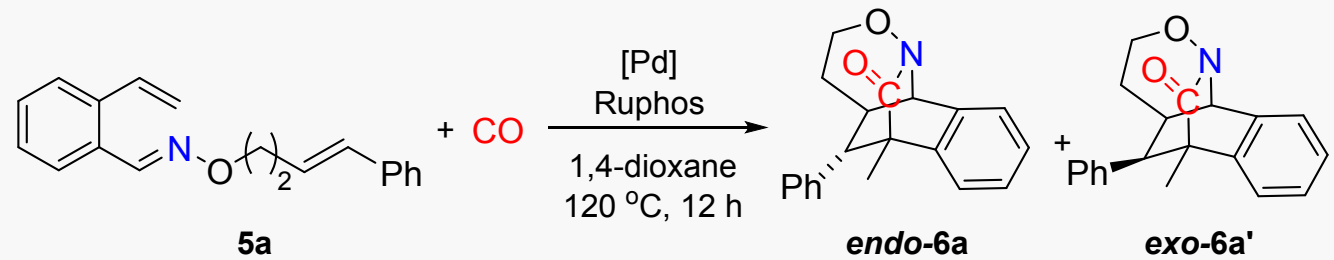

\begin{tabular}{cccc}
\hline Entry & Palladium catalyst & ${\text { Yield }(\%)^{b}}^{b}$ & endo/exo $^{b}$ \\
\hline 1 & $\mathrm{PdCl}_{2}$ & 57 & $91: 9$ \\
2 & {$\left[\mathrm{Pd}(\right.$ allyl $) \mathrm{Cl}_{2}$} & 37 & $91: 9$ \\
3 & $\mathrm{Pd}\left(\mathrm{MeCN}_{2} \mathrm{Cl}_{2}\right.$ & 41 & $91: 9$ \\
4 & $\mathrm{Pd}(\mathrm{PhCN})_{2} \mathrm{Cl}_{2}$ & 65 & $91: 9$ \\
5 & $\mathrm{Pd}(\mathrm{cod}) \mathrm{Cl}_{2}$ & 12 & $91: 9$ \\
\hline
\end{tabular}

${ }^{a}$ Reaction conditions: 5a $(0.5 \mathrm{mmol})$, palladium catalyst $(5 \mathrm{~mol} \%)$, Ruphos (12 mol \%), $\mathrm{NH}_{4} \mathrm{Cl}(5 \mathrm{~mol} \%), \mathrm{CO}$ (20 atm), 1,4-dioxane (1.5 mL), $120{ }^{\circ} \mathrm{C}, 12$ hours. ${ }^{b}$ Yields and ratios of endo/exo were determined by GC-analysis using $n$-tetradecane as an internal standard. 
Table S7. Screening of solvents for the synthesis of $6 a^{a}$

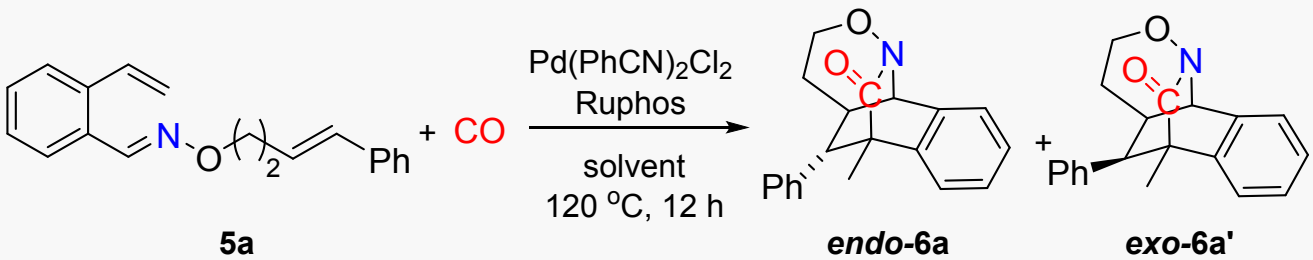

\begin{tabular}{cccc}
\hline Entry & Solvent & ${\text { Yield }(\%)^{b}}^{b}$ & endo/exo $^{b}$ \\
\hline 1 & Anisole & 33 & $90: 10$ \\
2 & THF & $70(70)^{c}$ & $91: 9$ \\
3 & DMF & 21 & $89: 11$ \\
4 & DCM & trace & - \\
5 & Toluene & trace & - \\
6 & NMP & 43 & $88: 12$ \\
7 & $1,4-$ dixoane & 65 & $91: 9$ \\
8 & MeCN & trace & - \\
9 & TBME & trace & - \\
\hline
\end{tabular}

${ }^{a}$ Reaction conditions: 5a $(0.5 \mathrm{mmol}), \mathrm{Pd}(\mathrm{PhCN})_{2} \mathrm{Cl}_{2}(5 \mathrm{~mol} \%)$, Ruphos $(12 \mathrm{~mol} \%), \mathrm{NH}_{4} \mathrm{Cl}$ (5 mol \%), CO (20 atm), solvent $(1.5 \mathrm{~mL}), 120{ }^{\circ} \mathrm{C}, 12$ hours. ${ }^{b}$ Yields and ratios of endo/exo were determined by GC-analysis using $n$-tetradecane as an internal standard. ${ }^{c}$ Isolated yield.

Table S8. Screening of ligands for the synthesis of $6 a^{a}$

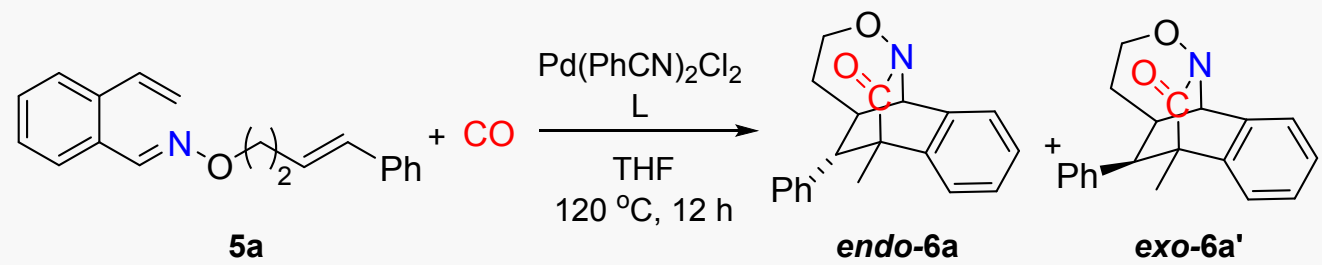

\begin{tabular}{cccc}
\hline Entry & Ligand (mol \%) & ${\text { Yield }(\%)^{b}}^{b}$ & endo/exo $^{b}$ \\
\hline 1 & Xantphos (6) & 0 & - \\
2 & Ruphos (12) & 70 & $91: 9$ \\
3 & Xphos (12) & 40 & $85: 15$ \\
4 & $t$-Bu-Xphos (12) & 28 & $85: 15$ \\
5 & Mephos (12) & trace & - \\
6 & L1 (12) & trace & - \\
7 & L2 (12) & trace & - \\
8 & Davephos (12) & 45 & $88: 12$ \\
9 & Brettphos (12) & 11 & $85: 15$ \\
10 & Ruphos (6) & 70 & $91: 9$ \\
\hline
\end{tabular}

${ }^{a}$ Reaction conditions: 5a $(0.5 \mathrm{mmol}), \mathrm{Pd}(\mathrm{PhCN})_{2} \mathrm{Cl}_{2}(5 \mathrm{~mol} \%)$, Ligand (6 or $\left.12 \mathrm{~mol} \%\right)$, $\mathrm{NH}_{4} \mathrm{Cl}(5 \mathrm{~mol} \%)$, CO $(20 \mathrm{~atm})$, THF $(1.5 \mathrm{~mL}), 120{ }^{\circ} \mathrm{C}, 12$ hours. ${ }^{b}$ Yields and ratios of endo/exo were determined by GC-analysis using $n$-tetradecane as an internal standard. 
Table S9. Screening of CO pressure for the synthesis of $6 \mathrm{a}^{a}$

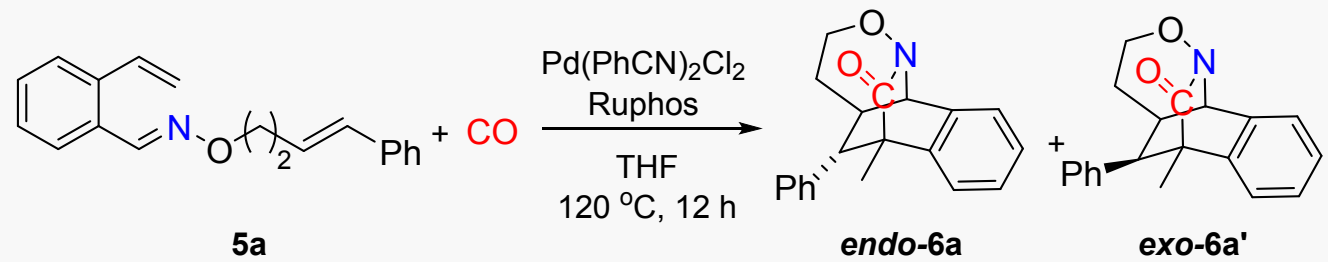

\begin{tabular}{cccc}
\hline Entry & $\mathrm{CO}(\mathrm{atm})$ & ${\text { Yield }(\%)^{b}}^{b}$ & endo/exo $^{b}$ \\
\hline 1 & 5 & trace & - \\
2 & 10 & 46 & $91: 9$ \\
3 & 20 & 70 & $91: 9$ \\
4 & 30 & 70 & $91: 9$ \\
\hline
\end{tabular}

${ }^{a}$ Reaction conditions: 5a $(0.5 \mathrm{mmol}), \mathrm{Pd}(\mathrm{PhCN})_{2} \mathrm{Cl}_{2}(5 \mathrm{~mol} \%)$, Ruphos $(12 \mathrm{~mol} \%), \mathrm{NH}_{4} \mathrm{Cl}$ $(5 \mathrm{~mol} \%)$, THF $(1.5 \mathrm{~mL}), 120{ }^{\circ} \mathrm{C}, 12$ hours. ${ }^{b}$ Yields and ratios of endo/exo were determined by GC-analysis using $n$-tetradecane as an internal standard.

Table S10. Screening of temperature for the synthesis of $6 a^{a}$

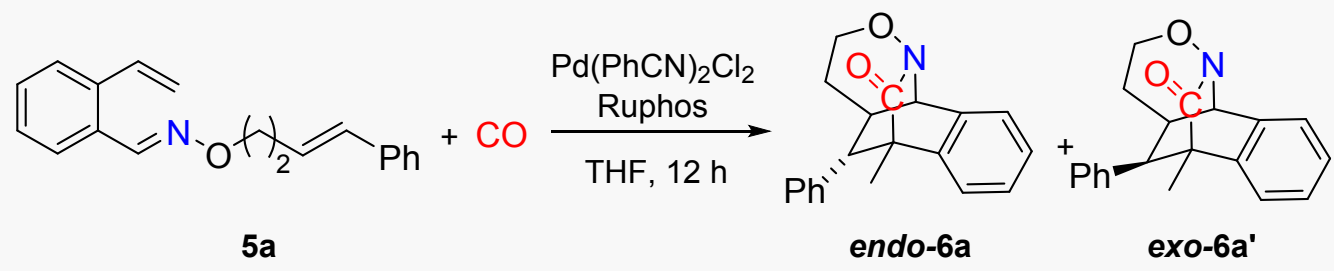

\begin{tabular}{cccc}
\hline Entry & temperature $\left({ }^{\circ} \mathrm{C}\right)$ & Yield $(\%)^{b}$ & endo/exo $^{b}$ \\
\hline 1 & 80 & trace & - \\
2 & 100 & 58 & $91: 9$ \\
3 & 120 & 70 & $91: 9$ \\
4 & 140 & 68 & $91: 9$ \\
\hline
\end{tabular}

${ }^{a}$ Reaction conditions: 5a $(0.5 \mathrm{mmol}), \mathrm{Pd}(\mathrm{PhCN})_{2} \mathrm{Cl}_{2}(5 \mathrm{~mol} \%)$, Ruphos $(12 \mathrm{~mol} \%), \mathrm{NH}_{4} \mathrm{Cl}$ ( $5 \mathrm{~mol} \%)$, CO (20 atm), THF (1.5 mL), 12 hours. ${ }^{b}$ Yields and ratios of endo/exo were determined by GC-analysis using $n$-tetradecane as an internal standard. 


\section{Experimental characterization data for the starting materials}

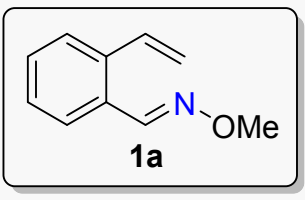

(E)-2-vinylbenzaldehyde $\boldsymbol{O}$-methyl oxime (1a): The title compound was prepared according to the general procedure (Method A) and purified by flash column chromatography (petroleum ether/ethyl acetate $=50 / 1)$ to give a light colorless oil $(1.21 \mathrm{~g}, 75 \%$ yield). ${ }^{1} \mathbf{H}$ NMR $\left(400 \mathrm{MHz}, \mathrm{CDCl}_{3}\right) \delta 3.99(\mathrm{~s}, 3 \mathrm{H}), 5.37\left(\mathrm{dd}, J_{l}=10.8 \mathrm{~Hz}, J_{2}=1.2 \mathrm{~Hz}, 1 \mathrm{H}\right)$, $5.60\left(\mathrm{dd}, J_{1}=17.2 \mathrm{~Hz}, J_{2}=1.2 \mathrm{~Hz}, 1 \mathrm{H}\right), 7.02\left(\mathrm{dd}, J_{l}=17.2 \mathrm{~Hz}, J_{2}=10.8 \mathrm{~Hz}, 1 \mathrm{H}\right)$, 7.25-7.29 (m, 1H), 7.32-7.36 (m, 1H), $7.46\left(\mathrm{dd}, J_{1}=7.6 \mathrm{~Hz}, J_{2}=0.8 \mathrm{~Hz}, 1 \mathrm{H}\right), 7.71$ $\left(\mathrm{dd}, J_{1}=7.6 \mathrm{~Hz}, J_{2}=0.8 \mathrm{~Hz}, 1 \mathrm{H}\right), 8.39(\mathrm{~s}, 1 \mathrm{H}) ;{ }^{13} \mathbf{C}$ NMR $\left(100 \mathrm{MHz}, \mathrm{CDCl}_{3}\right) \delta 62.2$, 117.9, 126.9, 127.1, 127.9, 129.4, 129.8, 134.0, 137.4, 147.3; HRMS (ESI) $m / z$ : $[\mathrm{M}+\mathrm{H}]^{+}$Calcd for $\mathrm{C}_{10} \mathrm{H}_{12} \mathrm{NO} 162.0919$, found 162.0916.

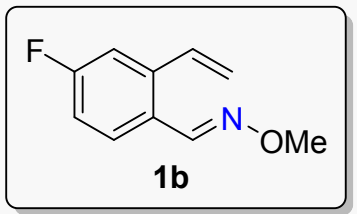

(E)-4-fluoro-2-vinylbenzaldehyde $O$-methyl oxime (1b): The title compound was prepared according to the general procedure (Method A) and purified by flash column chromatography (petroleum ether/ethyl acetate $=50 / 1$ ) to give a light colorless oil (1.40 g, 78\% yield). ${ }^{1} \mathbf{H}$ NMR (400 MHz, $\left.\mathrm{CDCl}_{3}\right) \delta 3.98(\mathrm{~s}, 3 \mathrm{H})$, $5.42(\mathrm{~d}, J=10.8 \mathrm{~Hz}, 1 \mathrm{H}), 5.62\left(\mathrm{dd}, J_{1}=17.2 \mathrm{~Hz}, J_{2}=0.4 \mathrm{~Hz}, 1 \mathrm{H}\right), 6.95-7.05(\mathrm{~m}, 2 \mathrm{H})$, $7.14\left(\mathrm{dd}, J_{1}=10.0 \mathrm{~Hz}, J_{2}=2.8 \mathrm{~Hz}, 1 \mathrm{H}\right), 7.70\left(\mathrm{dd}, J_{1}=8.4 \mathrm{~Hz}, J_{2}=5.6 \mathrm{~Hz}, 1 \mathrm{H}\right), 8.32$ $(\mathrm{s}, 1 \mathrm{H}) ;{ }^{13} \mathrm{C}$ NMR $\left(100 \mathrm{MHz}, \mathrm{CDCl}_{3}\right) \delta 62.2,113.1(\mathrm{~d}, J=20.0 \mathrm{~Hz}), 115.2(\mathrm{~d}, J=$ $20.0 \mathrm{~Hz}), 118.9,125.6(\mathrm{~d}, J=10.0 \mathrm{~Hz}), 129.2(\mathrm{~d}, J=10.0 \mathrm{~Hz}), 132.0(\mathrm{~d}, J=10 \mathrm{~Hz})$, $139.5(\mathrm{~d}, J=10 \mathrm{~Hz}), 146.3,163.7(\mathrm{~d}, J=240 \mathrm{~Hz}) ;{ }^{19} \mathbf{F}$ NMR $\left(376 \mathrm{MHz}, \mathrm{CDCl}_{3}\right) \delta$ -111.2; HRMS (ESI) $m / z$ : $[\mathrm{M}+\mathrm{H}]^{+}$Calcd for $\mathrm{C}_{10} \mathrm{H}_{11} \mathrm{NOF} 180.0825$, found 180.0833.

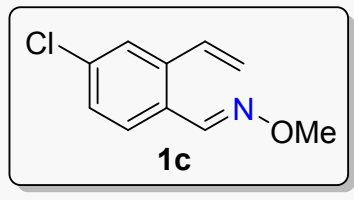

(E)-4-chloro-2-vinylbenzaldehyde $O$-methyl oxime (1c): The title compound was prepared according to the general procedure (Method A) and purified by flash column chromatography (petroleum ether/ethyl acetate $=50 / 1$ ) to give a light colorless oil (1.56 g, 80\% yield). ${ }^{1} \mathbf{H}$ NMR (400 MHz, $\left.\mathrm{CDCl}_{3}\right) \delta 3.98(\mathrm{~s}, 3 \mathrm{H}), 5.42$ (dd, $J_{1}=11.2$ $\left.\mathrm{Hz}, J_{2}=1.2 \mathrm{~Hz}, 1 \mathrm{H}\right), 5.62\left(\mathrm{dd}, J_{l}=17.2 \mathrm{~Hz}, J_{2}=0.8 \mathrm{~Hz}, 1 \mathrm{H}\right), 6.95\left(\mathrm{dd}, J_{l}=17.2 \mathrm{~Hz}\right.$, $\left.J_{2}=10.8 \mathrm{~Hz}, 1 \mathrm{H}\right), 7.22\left(\mathrm{dd}, J_{1}=8.4 \mathrm{~Hz}, J_{2}=2.0 \mathrm{~Hz}, 1 \mathrm{H}\right), 7.44(\mathrm{~d}, J=2.0 \mathrm{~Hz}, 1 \mathrm{H})$, 
$7.67(\mathrm{~d}, J=8.4 \mathrm{~Hz}, 1 \mathrm{H}), 8.31(\mathrm{~s}, 1 \mathrm{H}) ;{ }^{13} \mathrm{C}$ NMR $\left(100 \mathrm{MHz}, \mathrm{CDCl}_{3}\right) \delta$ 62.3, 119.1, $126.8,127.8,128.1,128.3,132.9,135.8,138.8,146.2$; HRMS (ESI) $m / z:[\mathrm{M}+\mathrm{H}]^{+}$ Calcd for $\mathrm{C}_{10} \mathrm{H}_{11} \mathrm{NOCl} 196.0529$, found 196.0526.

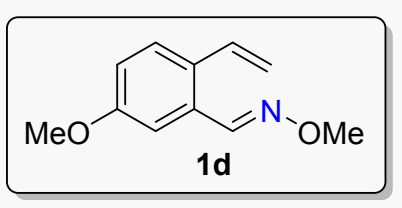

(E)-5-methoxy-2-vinylbenzaldehyde $O$-methyl oxime (1d): The title compound was prepared according to the general procedure (Method A) and purified by flash column chromatography (petroleum ether/ethyl acetate $=50 / 1$ ) to give a light colorless oil (1.05g, 55\% yield). ${ }^{1} \mathbf{H}$ NMR (400 MHz, $\left.\mathrm{CDCl}_{3}\right) \delta 3.76(\mathrm{~s}, 3 \mathrm{H}), 3.92$ (s, 3H), 5.21 (dd, $J=10.9,1.2 \mathrm{~Hz}, 1 \mathrm{H}), 5.45(\mathrm{dd}, J=17.2,1.2 \mathrm{~Hz}, 1 \mathrm{H}), 6.83-6.92(\mathrm{~m}, 2 \mathrm{H}), 7.18(\mathrm{~d}, J=$ $2.8 \mathrm{~Hz}, 1 \mathrm{H}), 7.33(\mathrm{~d}, J=8.7 \mathrm{~Hz}, 1 \mathrm{H}), 8.31(\mathrm{~s}, 1 \mathrm{H}) ;{ }^{13} \mathbf{C ~ N M R}\left(100 \mathrm{MHz}, \mathrm{CDCl}_{3}\right) \delta$ $55.4,62.1,110.0,116.1,117.1,128.0,130.2,130.3,133.1,146.9,159.1$.

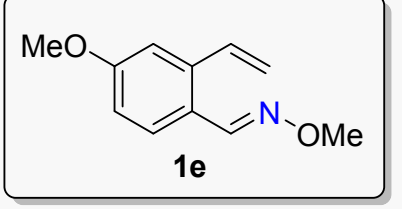

(E)-4-methoxy-2-vinylbenzaldehyde $O$-methyl oxime (1e): The title compound was prepared according to the general procedure (Method A) and purified by flash column chromatography (petroleum ether/ethyl acetate $=50 / 1$ ) to give a light colorless oil (1.65 g, 85\% yield). ${ }^{1} \mathbf{H}$ NMR $\left(400 \mathrm{MHz}, \mathrm{CDCl}_{3}\right) \delta 3.84(\mathrm{~s}$, $3 \mathrm{H}), 3.99(\mathrm{~s}, 3 \mathrm{H}), 5.27\left(\mathrm{dd}, J_{1}=10.8 \mathrm{~Hz}, J_{2}=1.2 \mathrm{~Hz}, 1 \mathrm{H}\right), 5.50\left(\mathrm{dd}, J_{1}=17.2 \mathrm{~Hz}, J_{2}=\right.$ $1.2 \mathrm{~Hz}, 1 \mathrm{H}), 6.90-7.00(\mathrm{~m}, 2 \mathrm{H}), 7.25$ (d, $J=2.8 \mathrm{~Hz}, 1 \mathrm{H}), 7.39$ (d, $J=8.8 \mathrm{~Hz}, 1 \mathrm{H})$, $8.38(\mathrm{~s}, 1 \mathrm{H}) ;{ }^{13} \mathrm{C}$ NMR $\left(100 \mathrm{MHz}, \mathrm{CDCl}_{3}\right) \delta 55.5,62.1,110.1,116.2,117.2,128.1$, 130.3, 130.4, 133.2, 147.0, 159.2; HRMS (ESI) $\mathrm{m} / z$ : $[\mathrm{M}+\mathrm{H}]^{+}$Calcd for $\mathrm{C}_{11} \mathrm{H}_{14} \mathrm{NO}_{2}$ 192.1025, found 192.1020 .

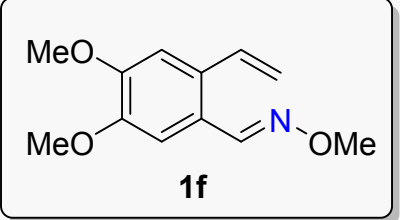

(E)-4,5-dimethoxy-2-vinylbenzaldehyde $O$-methyl oxime (1f): The title compound was prepared according to the general procedure (Method A) and purified by flash column chromatography (petroleum ether/ethyl acetate $=$ 50/1) to give a white solid (1.94 g, 88\% yield), m. p. $61-65^{\circ} \mathrm{C} .{ }^{1} \mathbf{H}$ NMR (400 MHz, $\left.\mathrm{CDCl}_{3}\right) \delta 3.92(\mathrm{~s}, 3 \mathrm{H}), 3.93(\mathrm{~s}, 3 \mathrm{H}), 3.97(\mathrm{~s}, 3 \mathrm{H}), 5.31\left(\mathrm{dd}, J_{1}=11.2 \mathrm{~Hz}, J_{2}=1.2 \mathrm{~Hz}\right.$, $1 \mathrm{H}), 5.52\left(\mathrm{dd}, J_{1}=17.2 \mathrm{~Hz}, J_{2}=1.2 \mathrm{~Hz}, 1 \mathrm{H}\right), 6.93(\mathrm{~s}, 1 \mathrm{H}), 6.95\left(\mathrm{dd}, J_{l}=17.2 \mathrm{~Hz}, J_{2}=\right.$ $10.8 \mathrm{~Hz}, 1 \mathrm{H}), 7.28(\mathrm{~s}, 1 \mathrm{H}), 8.37(\mathrm{~s}, 1 \mathrm{H}) ;{ }^{13} \mathbf{C ~ N M R}\left(100 \mathrm{MHz}, \mathrm{CDCl}_{3}\right) \delta 55.9,56.0$, 
62.0, 108.1, 108.6, 116.4, 122.1, 130.9, 133.1, 146.6, 149.0, 150.6; HRMS (ESI) $\mathrm{m} / z$ : $[\mathrm{M}+\mathrm{H}]^{+}$Calcd for $\mathrm{C}_{12} \mathrm{H}_{16} \mathrm{NO}_{3} 222.1130$, found 222.1127 .

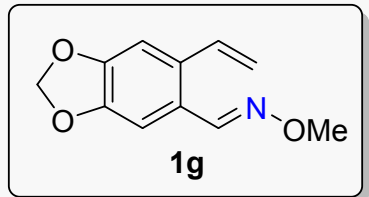

(E)-6-vinylbenzo[d][1,3]dioxole-5-carbaldehyde $O$-methyl oxime (1g): The title compound was prepared according to the general procedure (Method A) and purified by flash column chromatography (petroleum ether/ethyl acetate = $50 / 1)$ to give a light colorless oil (1.82 g, 89\% yield). ${ }^{1} \mathbf{H}$ NMR $\left(400 \mathrm{MHz}, \mathrm{CDCl}_{3}\right) \delta$ $3.88(\mathrm{~s}, 3 \mathrm{H}), 5.21\left(\mathrm{dd}, J_{1}=10.8 \mathrm{~Hz}, J_{2}=1.2 \mathrm{~Hz}, 1 \mathrm{H}\right), 5.42\left(\mathrm{dd}, J_{1}=17.2 \mathrm{~Hz}, J_{2}=0.8\right.$ $\mathrm{Hz}, 1 \mathrm{H}), 5.90$ (s, 2H), 6.83-6.90 (m, 2H), 7.18 (s, 1H), 8.26 (s, 1H); ${ }^{13} \mathrm{C}$ NMR (100 $\left.\mathrm{MHz}, \mathrm{CDCl}_{3}\right) \delta 62.1,101.6,105.7,106.1,116.6,123.7,132.6,133.1,146.5,147.8$, 149.5; HRMS (ESI) $m / z$ : $[\mathrm{M}+\mathrm{H}]^{+}$Calcd for $\mathrm{C}_{11} \mathrm{H}_{12} \mathrm{NO}_{3} 206.0817$, found: 206.0811 .

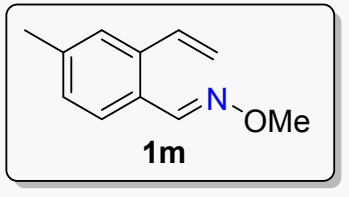

(E)-4-methyl-2-vinylbenzaldehyde $O$-methyl oxime (1m):

The title compound was prepared according to the general procedure (Method A) and purified by flash column chromatography (petroleum ether/ethyl acetate $=50 / 1$ ) to give a light colorless oil (1.22 g, 70\% yield). ${ }^{1} \mathbf{H}$ NMR (400 MHz, $\left.\mathrm{CDCl}_{3}\right) \delta 2.35$ (s, 3H), 3.97 (s, 3H), 5.34 $\left(\mathrm{dd}, J_{1}=11.2 \mathrm{~Hz}, J_{2}=1.2 \mathrm{~Hz}, 1 \mathrm{H}\right), 5.58\left(\mathrm{dd}, J_{1}=17.6 \mathrm{~Hz}, J_{2}=1.2 \mathrm{~Hz}, 1 \mathrm{H}\right), 7.00-7.09$ $(\mathrm{m}, 2 \mathrm{H}), 7.27(\mathrm{~s}, 1 \mathrm{H}), 7.61(\mathrm{~d}, J=8.0 \mathrm{~Hz}, 1 \mathrm{H}), 8.35(\mathrm{~s}, 1 \mathrm{H}) ;{ }^{13} \mathbf{C}$ NMR (100 MHz, $\left.\mathrm{CDCl}_{3}\right) \delta 21.5,62.0,117.6,126.6,127.0,127.4,128.9,134.1,137.3,139.9,147.3$; HRMS (ESI) $m / z$ : $[\mathrm{M}+\mathrm{H}]^{+}$Calcd for $\mathrm{C}_{11} \mathrm{H}_{14} \mathrm{NO} 176.1075$, found 176.1073.

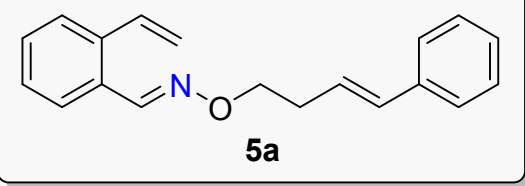

(E)-2-vinylbenzaldehyde

O-((E)-4-phenylbut-3-en-1-yl) oxime (5a): The title compound was prepared according to the general procedure (Method B) and purified by flash column chromatography (petroleum ether/ethyl acetate $=50 / 1$ ) to give a light colorless oil (0.82 g, 32\% yield, E,E/E,Z > 99:1). ${ }^{1} \mathbf{H}$ NMR $\left(400 \mathrm{MHz}, \mathrm{CDCl}_{3}\right) \delta 2.96$ $\left(\mathrm{dt}, J_{1}=7.2 \mathrm{~Hz}, J_{2}=1.2 \mathrm{~Hz}, 2 \mathrm{H}\right), 4.30(\mathrm{t}, J=7.2 \mathrm{~Hz}, 2 \mathrm{H}), 5.17\left(\mathrm{dd}, J_{1}=2.8 \mathrm{~Hz}, J_{2}=\right.$ $1.6 \mathrm{~Hz}, 1 \mathrm{H}), 5.36-5.40(\mathrm{~m}, 2 \mathrm{H}), 5.60\left(\mathrm{dd}, J_{l}=17.2 \mathrm{~Hz}, J_{2}=1.2 \mathrm{~Hz}, 1 \mathrm{H}\right), 7.03\left(\mathrm{dd}, J_{l}\right.$ $\left.=17.2 \mathrm{~Hz}, J_{2}=10.8 \mathrm{~Hz}, 1 \mathrm{H}\right), 7.24-7.29(\mathrm{~m}, 2 \mathrm{H}), 7.32-7.36(\mathrm{~m}, 3 \mathrm{H}), 7.45-7.48(\mathrm{~m}$, 3H), $7.70\left(\mathrm{dd}, J_{1}=7.6 \mathrm{~Hz}, J_{2}=1.6 \mathrm{~Hz}, 1 \mathrm{H}\right), 8.38(\mathrm{~s}, 1 \mathrm{H}) ;{ }^{13} \mathbf{C}$ NMR $(100 \mathrm{MHz}$, 
$\left.\mathrm{CDCl}_{3}\right) \delta 33.1,73.6,117.7,126.1,126.5,126.8,127.0,127.1,127.8,128.5,129.7$ 130.9, 131.9, 134.0, 137.3, 137.5, 147.3; HRMS (ESI) $m / z:[\mathrm{M}+\mathrm{Na}]^{+}$Calcd for $\mathrm{C}_{19} \mathrm{H}_{19} \mathrm{NNaO} 300.1359$, found 300.1360 .

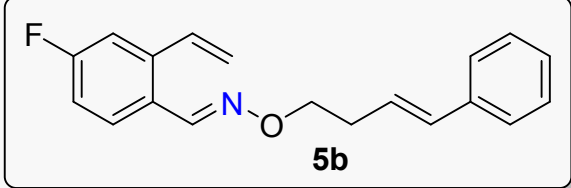

\section{(E)-4-fluoro-2-vinylbenzaldehyde}

$O$-((E)-4-phenylbut-3-en-1-yl) oxime (5b): The title compound was prepared according to the general procedure (Method B) and purified by

flash column chromatography (petroleum ether/ethyl acetate $=50 / 1$ ) to give a light colorless oil $(1.71 \mathrm{~g}, 29 \%$ yield, $E, E / E, Z=90: 10) .{ }^{1} \mathbf{H}$ NMR $\left(400 \mathrm{MHz}, \mathrm{CDCl}_{3}\right) \delta$ 2.55-2.73 (m, 2H), 4.18-4.23 (m, 2H), $5.36\left(\mathrm{dd}, J_{1}=11.2 \mathrm{~Hz}, J_{2}=0.8 \mathrm{~Hz}, 1 \mathrm{H}\right), 5.59$ $\left(\mathrm{dd}, J_{1}=17.6 \mathrm{~Hz}, J_{2}=1.2 \mathrm{~Hz}, 1 \mathrm{H}\right), 6.15-6.23(\mathrm{~m}, 1 \mathrm{H}), 6.39-6.44(\mathrm{~m}, 1 \mathrm{H}), 6.87-6.99$ (m, 2H), 7.07-7.30 (m, 6H), 7.59-7.67 (m, 1H), $8.26(\mathrm{~s}, 0.10 \mathrm{H}), 8.28(\mathrm{~s}, 0.90 \mathrm{H}) ;{ }^{13} \mathrm{C}$ NMR $\left(100 \mathrm{MHz}, \mathrm{CDCl}_{3}\right) \delta 33.0,73.6,113.2(\mathrm{~d}, J=22 \mathrm{~Hz}), 115.3(\mathrm{~d}, J=22 \mathrm{~Hz})$, 118.7, 125.7 (d, $J=3 \mathrm{~Hz}), 126.1,126.4,127.1,128.5,129.1$ (d, $J=8 \mathrm{~Hz}), 132.0$, $133.0(\mathrm{~d}, J=2 \mathrm{~Hz}), 137.5,139.5(\mathrm{~d}, J=8 \mathrm{~Hz}), 146.2,162.3(\mathrm{~d}, J=248 \mathrm{~Hz}) ;{ }^{19} \mathbf{F}$ NMR $\left(376 \mathrm{MHz}, \mathrm{CDCl}_{3}\right) \delta-111.3$; HRMS (ESI) $m / z$ : $[\mathrm{M}+\mathrm{H}]^{+}$Calcd for $\mathrm{C}_{19} \mathrm{H}_{19} \mathrm{FNO}$ 296.1445, found 296.1447.

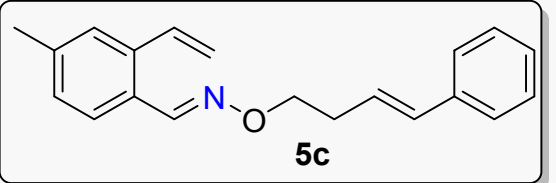

\section{(E)-4-methyl-2-vinylbenzaldehyde}

$O$-((E)-4-phenylbut-3-en-1-yl) oxime (5c): The title compound was prepared according to the general procedure (Method B) and purified by flash column chromatography (petroleum ether/ethyl acetate $=50 / 1$ ) to give a light colorless oil (2.04 g, 35\% yield, E,E/E,Z > 99:1). ${ }^{1} \mathbf{H}$ NMR (400 MHz, $\left.\mathrm{CDCl}_{3}\right) \delta 2.29$ $(\mathrm{s}, 3 \mathrm{H}), 2.58(\mathrm{q}, J=6.6 \mathrm{~Hz}, 2 \mathrm{H}), 4.22(\mathrm{t}, J=6.7 \mathrm{~Hz}, 2 \mathrm{H}), 5.28(\mathrm{dd}, J=10.9,1.2 \mathrm{~Hz}$, $1 \mathrm{H}), 5.54(\mathrm{dd}, J=17.4,1.2 \mathrm{~Hz}, 1 \mathrm{H}), 6.16-6.24(\mathrm{~m}, 1 \mathrm{H}), 6.42(\mathrm{~d}, J=15.9 \mathrm{~Hz}, 1 \mathrm{H})$, 6.94-7.03 (m, 2H), $7.13(\mathrm{t}, J=7.3 \mathrm{~Hz}, 1 \mathrm{H}), 7.20-7.30(\mathrm{~m}, 5 \mathrm{H}), 7.56(\mathrm{~d}, J=7.9 \mathrm{~Hz}$, 1H), $8.32(\mathrm{~s}, 1 \mathrm{H}) ;{ }^{13} \mathrm{C}$ NMR (100 MHz, $\left.\mathrm{CDCl}_{3}\right) \delta$ 21.0, 33.0, 73.5, 117.4, 126.1, $126.6,126.7,127.0,127.1,127.3,128.5,128.8,131.9,134.1,137.1,137.5,139.7$, 147.3; HRMS (ESI) $m / z$ : $[\mathrm{M}+\mathrm{Na}]^{+}$Calcd for $\mathrm{C}_{20} \mathrm{H}_{21} \mathrm{NNaO} 314.1515$, found 314.1511 . 


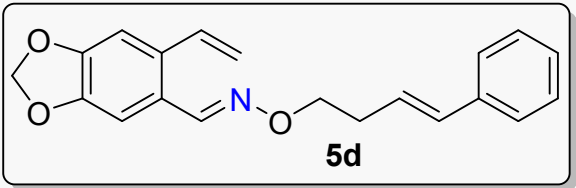

(E)-6-vinylbenzo[d][1,3]dioxole-5-carbaldeh yde $O$-((E)-4-phenylbut-3-en-1-yl) oxime (5d): The title compound was prepared according to the general procedure (Method B) and purified by flash column chromatography (petroleum ether/ethyl acetate $=50 / 1$ ) to give a light colorless oil (1.41 g, 22\% yield, $E, E / E, Z>61: 39) .{ }^{1} \mathbf{H}$ NMR (400 MHz, $\left.\mathrm{CDCl}_{3}\right) \delta 2.54-2.60(\mathrm{~m}, 1 \mathrm{H}), 2.67-2.72(\mathrm{~m}, 1 \mathrm{H}), 4.21\left(\mathrm{dd}, J_{1}=14.0 \mathrm{~Hz}, J_{2}=6.8 \mathrm{~Hz}\right.$, 2H), 5.20-5.24 (m, 1H), 5.41-5.46 (m, 1H), 5.64-5.68 (m, 0.44H), $5.90(\mathrm{~s}, 2 \mathrm{H})$, 6.15-6.23 (m, 0.61H), 6.40-6.49 (m, 1H), 6.82-6.91 (m, 2H), 7.11-7.30 (m, 6H), 8.28 $(\mathrm{s}, 0.39 \mathrm{H}), 8.30(\mathrm{~s}, 0.61 \mathrm{H}) ;{ }^{13} \mathrm{C}$ NMR $\left(100 \mathrm{MHz}, \mathrm{CDCl}_{3}\right) \delta$ 28.8, 33.0, 73.5, 73.6, $101.4,105.6,105.9,106.0,116.4,123.7,126.1,126.5,126.7,127.1,128.2,128.2$, $128.5,128.7,130.9,131.9,132.4,133.0,137.5,146.4,146.4,147.7,149.3$; HRMS (ESI) $m / z:[\mathrm{M}+\mathrm{H}]^{+}$Calcd for $\mathrm{C}_{20} \mathrm{H}_{20} \mathrm{NO}_{3} 322.1438$, found 322.1443 .

(E)-2-vinylbenzaldehyde $O$-(4-((E)-4-fluorophenyl)but-3-en-1-yl) oxime (5e): The

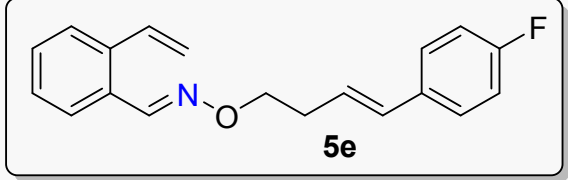
title compound was prepared according to the general procedure (Method B) and purified by flash column chromatography (petroleum ether/ethyl acetate $=50 / 1)$ to give a light colorless oil $(2.00 \mathrm{~g}, 34 \%$ yield, $E, E / E, Z>$ 99:1). ${ }^{1} \mathbf{H}$ NMR (400 MHz, $\left.\mathrm{CDCl}_{3}\right) \delta 2.54-2.60(\mathrm{~m}, 2 \mathrm{H}), 4.22(\mathrm{t}, J=6.4 \mathrm{~Hz}, 2 \mathrm{H}), 5.32$ $\left(\mathrm{dd}, J_{1}=10.8 \mathrm{~Hz}, J_{2}=1.2 \mathrm{~Hz}, 1 \mathrm{H}\right), 5.57\left(\mathrm{dd}, J_{1}=17.6 \mathrm{~Hz}, J_{2}=1.2 \mathrm{~Hz}, 1 \mathrm{H}\right), 6.07-6.15$ (m, 1H), 6.36-6.40 (m, 1H), 6.87-7.03 (m, 3H), 7.18-7.29 (m, 4H), $7.41\left(\mathrm{dd}, J_{l}=8.0\right.$ $\left.\mathrm{Hz}, J_{2}=1.6 \mathrm{~Hz}, 1 \mathrm{H}\right), 7.67\left(\mathrm{dd}, J_{1}=8.0 \mathrm{~Hz}, J_{2}=1.6 \mathrm{~Hz}, 1 \mathrm{H}\right), 8.35(\mathrm{~s}, 1 \mathrm{H}) ;{ }^{13} \mathbf{C} \mathbf{~ N M R}$ $\left(100 \mathrm{MHz}, \mathrm{CDCl}_{3}\right) \delta 33.0,73.5,115.4(\mathrm{~d}, J=21 \mathrm{~Hz}), 117.7,126.3(\mathrm{~d}, J=2 \mathrm{~Hz})$, 126.8, 127.0, 127.5 (d, $J=8 \mathrm{~Hz}), 127.8,129.4,129.7,130.7,133.7$ (d, $J=3 \mathrm{~Hz})$, 134.0, 137.3, 147.3, $160.8(\mathrm{~d}, J=244 \mathrm{~Hz}) ;{ }^{19} \mathbf{F}$ NMR $\left(376 \mathrm{MHz}, \mathrm{CDCl}_{3}\right) \delta-115.4$; HRMS (ESI) $m / z$ : [M+H] $]^{+}$Calcd for $\mathrm{C}_{19} \mathrm{H}_{19} \mathrm{FNO} 296.1445$, found 296.1441.

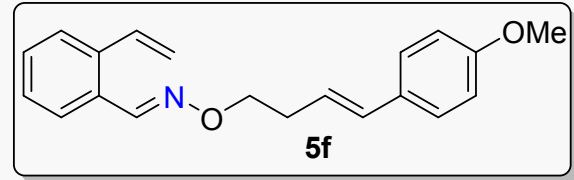

(E)-2-vinylbenzaldehyde

Methoxy)but-3-en-1-yl) oxime (5f): The title compound was prepared according to the general procedure (Method B) and purified by flash column chromatography (petroleum 
ether/ethyl acetate $=50 / 1)$ to give a light colorless oil $(2.02 \mathrm{~g}, 33 \%$ yield, $E, E / E, Z>$ 99:1). ${ }^{1} \mathbf{H}$ NMR $\left(400 \mathrm{MHz}, \mathrm{CDCl}_{3}\right) \delta 2.52-2.57(\mathrm{~m}, 2 \mathrm{H}), 3.70(\mathrm{~s}, 3 \mathrm{H}), 4.21(\mathrm{t}, J=6.8$ $\mathrm{Hz}, 2 \mathrm{H}), 5.31\left(\mathrm{dd}, J_{l}=10.8 \mathrm{~Hz}, J_{2}=1.2 \mathrm{~Hz}, 1 \mathrm{H}\right), 5.56\left(\mathrm{dd}, J_{l}=17.2 \mathrm{~Hz}, J_{2}=1.2 \mathrm{~Hz}\right.$, $1 \mathrm{H}), 6.00-6.07(\mathrm{~m}, 1 \mathrm{H}), 6.33-6.38(\mathrm{~m}, 1 \mathrm{H}), 6.74-6.76(\mathrm{~m}, 2 \mathrm{H}), 7.66\left(\mathrm{dd}, J_{1}=17.2 \mathrm{~Hz}\right.$, $\left.J_{2}=10.8 \mathrm{~Hz}, 1 \mathrm{H}\right), 7.15-7.27(\mathrm{~m}, 4 \mathrm{H}), 7.37-7.40(\mathrm{~m}, 1 \mathrm{H}), 7.02\left(\mathrm{dd}, J_{1}=7.6 \mathrm{~Hz}, J_{2}=\right.$ $1.6 \mathrm{~Hz}, 1 \mathrm{H}), 8.34(\mathrm{~s}, 1 \mathrm{H}) ;{ }^{13} \mathbf{C}$ NMR (100 MHz, $\left.\mathrm{CDCl}_{3}\right) \delta$ 33.0, 55.3, 73.8, 113.9, $117.7,124.2,126.8,127.0,127.2,127.8,129.5,129.7,130.4,131.3,134.0,137.3$, 147.2, 158.9; HRMS (ESI) $m / z$ : $[\mathrm{M}+\mathrm{Na}]^{+}$Calcd for $\mathrm{C}_{20} \mathrm{H}_{21} \mathrm{NNaO}_{2} 330.1464$, found 330.1457 .

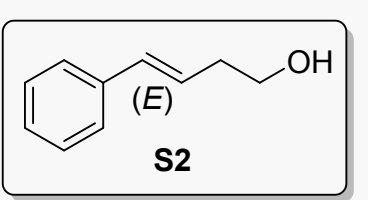

5H); ${ }^{13} \mathrm{C}$ NMR $\left(100 \mathrm{MHz}, \mathrm{CDCl}_{3}\right) \delta 36.4,62.0,126.1,126.3,127.3,128.6,132.9$, 137.2.

(E)-4-phenylbut-3-en-1-ol (S2) ${ }^{6}$ : ${ }^{1} \mathrm{H} \quad$ NMR $(400 \mathrm{MHz}$, $\left.\mathrm{CDCl}_{3}\right) \delta 2.42(\mathrm{q}, J=6.6 \mathrm{~Hz}, 2 \mathrm{H}), 3.69(\mathrm{t}, J=6.3 \mathrm{~Hz}, 2 \mathrm{H})$, 6.10-6.17 (m, 1H), $6.43(\mathrm{~d}, J=15.8 \mathrm{~Hz}, 1 \mathrm{H}), 7.13-7.30(\mathrm{~m}$, 


\section{Experimental characterization data for the products}

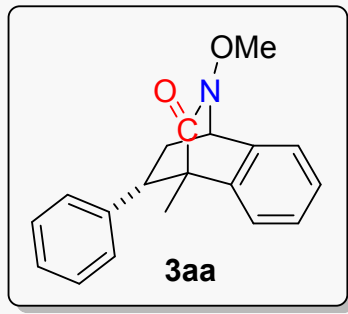

10-Methoxy-4-methyl-3-phenyl-1,2,3,4-tetrahydro-1,4-(epi minomethano)naphthalen-9-one (3aa): the title compound was prepared according to general procedure and purified by column chromatography (petroleum ether/ethyl acetate $=5 / 1$ to $3 / 1)$ to give a white solid (122 $\mathrm{mg}, 83 \%$ yield, m.p.

126-127 $\left.{ }^{\circ} \mathrm{C}\right) .{ }^{1} \mathbf{H}$ NMR $\left(400 \mathrm{MHz}, \mathrm{CDCl}_{3}\right) \delta 1.31(\mathrm{~s}, 3 \mathrm{H}), 1.77-1.82(\mathrm{~m}, 1 \mathrm{H})$, 2.93-3.00 (m, 1H), $3.17\left(\mathrm{dd}, J_{l}=10.0 \mathrm{~Hz}, J_{2}=4.8 \mathrm{~Hz}, 1 \mathrm{H}\right), 3.82(\mathrm{~s}, 3 \mathrm{H}), 4.95\left(\mathrm{dd}, J_{l}\right.$ $\left.=4.0 \mathrm{~Hz}, J_{2}=2.0 \mathrm{~Hz}, 1 \mathrm{H}\right), 6.28(\mathrm{~d}, J=7.2 \mathrm{~Hz}, 2 \mathrm{H}), 6.95(\mathrm{~d}, J=7.6 \mathrm{~Hz}, 1 \mathrm{H})$, 7.04-7.08 (m, 2H), 7.10-7.14 (m, 1H), 7.25-7.29 (m, 1H), 7.33-7.39 (m, 2H); ${ }^{13} \mathrm{C}$ NMR $\left(100 \mathrm{MHz}, \mathrm{CDCl}_{3}\right) \delta 14.2,38.2,46.2,52.7,60.7,63.4,121.5,125.0,127.0$, $127.4,127.9,128.0,128.5,137.7,140.1,140.6,173.4$; HRMS (ESI) $m / z:[\mathrm{M}+\mathrm{H}]^{+}$ Calcd for $\mathrm{C}_{19} \mathrm{H}_{20} \mathrm{NO}_{2} 294.1494$, found 294.1494.

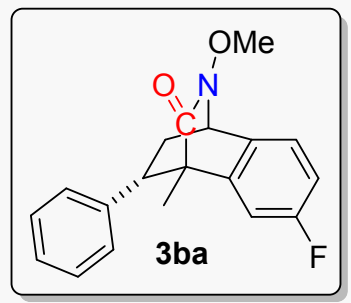

6-Fluoro-10-methoxy-4-methyl-3-phenyl-1,2,3,4-tetrahydr o-1,4-(epiminomethano)naphthalen-9-one (3ba): the title compound was prepared according to general procedure and purified by column chromatography (petroleum ether/ethyl acetate $=5 / 1$ to $3 / 1)$ to give a white solid $(106 \mathrm{mg}, 68 \%$ yield, m.p. $\left.123-124{ }^{\circ} \mathrm{C}\right) .{ }^{1} \mathbf{H}$ NMR $\left(400 \mathrm{MHz}, \mathrm{CDCl}_{3}\right) \delta 1.29$ (s, 3H), 1.79-1.84 (m, 1H), 2.93-3.00 (m, 1H), $3.18\left(\mathrm{dd}, J_{1}=10.4 \mathrm{~Hz}, J_{2}=4.8 \mathrm{~Hz}, 1 \mathrm{H}\right), 3.82(\mathrm{~s}, 3 \mathrm{H}), 4.94\left(\mathrm{dd}, J_{1}\right.$ $\left.=3.6 \mathrm{~Hz}, J_{2}=1.6 \mathrm{~Hz}, 1 \mathrm{H}\right), 6.32(\mathrm{~d}, J=7.2 \mathrm{~Hz}, 2 \mathrm{H}), 6.67\left(\mathrm{dd}, J_{1}=9.2 \mathrm{~Hz}, J_{2}=2.8 \mathrm{~Hz}\right.$, 1H), 7.01-7.06 (m, 1H), 7.08-7.12 (m, 2H), 7.13-7.18 (m, 1H), $7.35\left(\mathrm{dd}, J_{1}=8.0 \mathrm{~Hz}\right.$, $\left.J_{2}=4.8 \mathrm{~Hz}, 1 \mathrm{H}\right) ;{ }^{13} \mathrm{C}$ NMR $\left(100 \mathrm{MHz}, \mathrm{CDCl}_{3}\right) \delta 14.2,38.2,46.0,53.0(\mathrm{~d}, J=10 \mathrm{~Hz})$, 60.2, 63.6, 113.0 (d, $J=20 \mathrm{~Hz}), 113.9$ (d, $J=20 \mathrm{~Hz}), 122.9$ (d, $J=10 \mathrm{~Hz}), 127.3$, 128.2, 128.5, $136.0(\mathrm{~d}, J=10 \mathrm{~Hz}), 140.2,140.5(\mathrm{~d}, J=10 \mathrm{~Hz}), 161.6(\mathrm{~d}, J=244 \mathrm{~Hz})$, 173.0; ${ }^{19}$ F NMR $\left(376 \mathrm{MHz}, \mathrm{CDCl}_{3}\right) \delta-113.3$; HRMS (ESI) $m / z:[\mathrm{M}+\mathrm{H}]^{+}$Calcd for $\mathrm{C}_{19} \mathrm{H}_{19} \mathrm{NO}_{2} \mathrm{~F}$ 312.1400, found 312.1397.

6-Chloro-10-methoxy-4-methyl-3-phenyl-1,2,3,4-tetrahydro-1,4-(epiminomethan o)naphthalen-9-one (3ca): the title compound was prepared according to general 


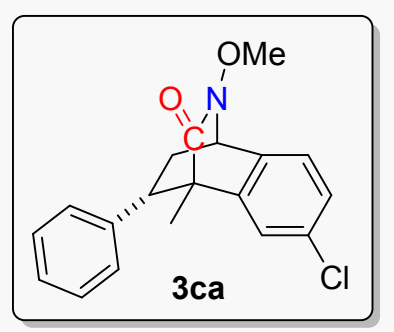

procedure and purified by column chromatography (petroleum ether/ethyl acetate $=5 / 1$ to $3 / 1$ ) to give a white solid (103 mg, 63\% yield, m.p. $\left.189-193{ }^{\circ} \mathrm{C}\right) .{ }^{1} \mathbf{H}$ NMR (400 $\left.\mathrm{MHz}, \mathrm{CDCl}_{3}\right) \delta 1.29(\mathrm{~s}, 3 \mathrm{H}), 1.79-1.84(\mathrm{~m}, 1 \mathrm{H}), 2.94-3.00$ $(\mathrm{m}, 1 \mathrm{H}), 3.18\left(\mathrm{dd}, J_{1}=10.0 \mathrm{~Hz}, J_{2}=4.8 \mathrm{~Hz}, 1 \mathrm{H}\right), 3.82(\mathrm{~s}$, $3 \mathrm{H}), 4.93\left(\mathrm{dd}, J_{1}=4.0 \mathrm{~Hz}, J_{2}=2.0 \mathrm{~Hz}, 1 \mathrm{H}\right), 6.33(\mathrm{~d}, J=7.2 \mathrm{~Hz}, 2 \mathrm{H}), 6.94(\mathrm{~d}, J=1.6$ $\mathrm{Hz}, 1 \mathrm{H}), 7.09-7.18(\mathrm{~m}, 3 \mathrm{H}), 7.31-7.36(\mathrm{~m}, 2 \mathrm{H}) ;{ }^{13} \mathbf{C} \mathbf{N M R}\left(100 \mathrm{MHz}, \mathrm{CDCl}_{3}\right) \delta 14.2$, $38.0,46.0,52.9,60.2,63.6,122.8,125.5,127.3,127.4,128.2,128.5,134.1,138.6$, 140.0, 140.2, 172.8; HRMS (ESI) $m / z$ : $[\mathrm{M}+\mathrm{H}]^{+}$Calcd for $\mathrm{C}_{19} \mathrm{H}_{19} \mathrm{NO}_{2} \mathrm{Cl} 328.1104$, found 328.1104 .

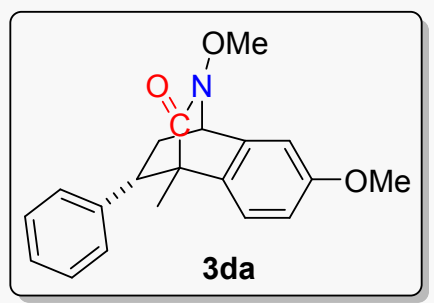

7,10-dimethoxy-4-methyl-3-phenyl-1,2,3,4-tetrahydro1,4-(epiminomethano)naphthalen-9-one (3da): the title compound was prepared according to general procedure and purified by column chromatography (petroleum ether/ethyl acetate $=5 / 1$ to $3 / 1)$ to give a white solid (111 mg, $68 \%$ yield, m.p. $\left.126-130{ }^{\circ} \mathrm{C}\right) .{ }^{1} \mathbf{H}$ NMR $\left(400 \mathrm{MHz}, \mathrm{CDCl}_{3}\right) \delta 1.27(\mathrm{~s}, 3 \mathrm{H})$, $1.75-1.80(\mathrm{~m}, 1 \mathrm{H}), 2.91-2.98(\mathrm{~m}, 1 \mathrm{H}), 3.14\left(\mathrm{dd}, J_{I}=10.4 \mathrm{~Hz}, J_{2}=4.8 \mathrm{~Hz}, 1 \mathrm{H}\right), 3.81(\mathrm{~s}$, $3 \mathrm{H}), 3.87(\mathrm{~s}, 3 \mathrm{H}), 4.87\left(\mathrm{dd}, J_{l}=3.6 \mathrm{~Hz}, J_{2}=1.6 \mathrm{~Hz}, 1 \mathrm{H}\right), 6.32-6.4(\mathrm{~m}, 2 \mathrm{H}), 6.77(\mathrm{dd}$, $\left.J_{1}=8.4 \mathrm{~Hz}, J_{2}=2.4 \mathrm{~Hz}, 1 \mathrm{H}\right), 6.84(\mathrm{~d}, J=8.4 \mathrm{~Hz}, 1 \mathrm{H}), 6.96(\mathrm{~d}, J=2.4 \mathrm{~Hz}, 1 \mathrm{H})$, 7.06-7.13 (m, 3H); ${ }^{13} \mathbf{C}$ NMR (100 MHz, $\left.\mathrm{CDCl}_{3}\right) \delta 14.2,38.2,46.2,51.9,55.5,60.8$, 63.4, 108.2, 112.2, 126.0, 126.9, 127.9, 128.6, 129.5, 140.8, 141.2, 159.1, 173.7; HRMS (ESI) $m / z$ : $[\mathrm{M}+\mathrm{Na}]^{+}$Calcd for $\mathrm{C}_{20} \mathrm{H}_{21} \mathrm{NNaO}_{3} 346.1414$, found 346.1409 .

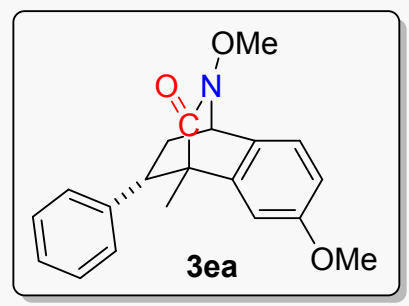

6,10-Dimethoxy-4-methyl-3-phenyl-1,2,3,4-tetrahydro1,4-(epiminomethano)naphthalen-9-one (3ea): the title compound was prepared according to general procedure and purified by column chromatography (petroleum ether/ethyl acetate $=5 / 1$ to $3 / 1)$ to give a white solid (124 mg, 77\% yield, m.p. $\left.177-180{ }^{\circ} \mathrm{C}\right) .{ }^{1} \mathbf{H}$ NMR $\left(400 \mathrm{MHz}, \mathrm{CDCl}_{3}\right) \delta 1.27(\mathrm{~s}, 3 \mathrm{H})$, $1.75-1.80(\mathrm{~m}, 1 \mathrm{H}), 2.91-2.98(\mathrm{~m}, 1 \mathrm{H}), 3.14\left(\mathrm{dd}, J_{I}=10.4 \mathrm{~Hz}, J_{2}=5.2 \mathrm{~Hz}, 1 \mathrm{H}\right), 3.82(\mathrm{~s}$, $3 \mathrm{H}), 3.87$ (s, 3H), 4.87 (dd, $\left.J_{1}=4.0 \mathrm{~Hz}, J_{2}=2.0 \mathrm{~Hz}, 1 \mathrm{H}\right), 6.33$ (d, $\left.J=7.2 \mathrm{~Hz} 2 \mathrm{H}\right)$, 
$6.77\left(\mathrm{dd}, J_{1}=8.0 \mathrm{~Hz}, J_{2}=2.4 \mathrm{~Hz}, 1 \mathrm{H}\right), 6.84(\mathrm{~d}, J=8.0 \mathrm{~Hz}, 1 \mathrm{H}), 6.96(\mathrm{~d}, J=2.4 \mathrm{~Hz}$, 1H), 7.06-7.10 (m, 2H), 7.11-7.16 (m, 1H); $\left.{ }^{13} \mathbf{C ~ N M R ~ ( 1 0 0 ~ M H z , ~} \mathrm{CDCl}_{3}\right) \delta 14.3,38.3$, $46.4,52.0,55.6,61.0,63.5,108.3,112.3,126.1,127.0,128.0,128.6,129.6,140.8$, 141.4, 159.2, 173.8; HRMS (ESI) $m / z$ : $[\mathrm{M}+\mathrm{Na}]^{+}$Calcd for $\mathrm{C}_{20} \mathrm{H}_{21} \mathrm{NNaO}_{3} 346.1414$, found 346.1411 .

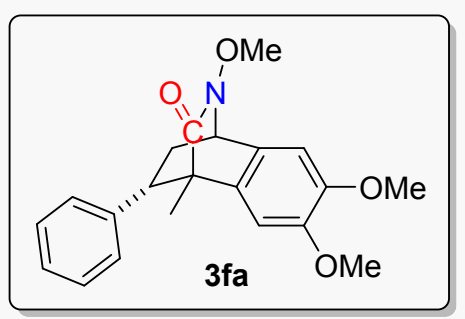

\section{6,7,10-Trimethoxy-4-methyl-3-phenyl-1,2,3,4-tetrahy} dro-1,4-(epiminomethano)naphthalen-9-one (3fa): the title compound was prepared according to general procedure and purified by column chromatography (petroleum ether/ethyl acetate $=5 / 1$ to $3 / 1$ ) to give a white solid (125 mg, 71\% yield, m.p. $\left.174-177^{\circ} \mathrm{C}\right) .{ }^{1} \mathbf{H}$ NMR $\left(400 \mathrm{MHz}, \mathrm{CDCl}_{3}\right) \delta$ $1.29(\mathrm{~s}, 3 \mathrm{H}), 1.76-1.81(\mathrm{~m}, 1 \mathrm{H}), 2.91-2.98(\mathrm{~m}, 1 \mathrm{H}), 3.14\left(\mathrm{dd}, J_{1}=10.4 \mathrm{~Hz}, J_{2}=4.8 \mathrm{~Hz}\right.$, $1 \mathrm{H}), 3.78(\mathrm{~s}, 3 \mathrm{H}), 3.83(\mathrm{~s}, 3 \mathrm{H}), 3.98(\mathrm{~s}, 3 \mathrm{H}), 4.86\left(\mathrm{dd}, J_{l}=3.6 \mathrm{~Hz}, J_{2}=1.6 \mathrm{~Hz}, 1 \mathrm{H}\right)$, $6.33(\mathrm{~d}, J=7.2 \mathrm{~Hz}, 2 \mathrm{H}), 6.49(\mathrm{~s}, 1 \mathrm{H}), 6.97(\mathrm{~s}, 1 \mathrm{H}), 7.06-7.16(\mathrm{~m}, 3 \mathrm{H}) ;{ }^{13} \mathbf{C}$ NMR $(100$ $\left.\mathrm{MHz}, \mathrm{CDCl}_{3}\right) \delta 14.5,38.6,46.3,52.4,56.3,56.4,60.6,63.5,105.8,109.2,127.0$, 128.0, 128.6, 129.9, 132.6, 140.8, 148.3, 148.7, 174.1; HRMS (ESI) $m / z:[\mathrm{M}+\mathrm{Na}]^{+}$ Calcd for $\mathrm{C}_{21} \mathrm{H}_{23} \mathrm{NNaO}_{4} 376.1519$, found 376.1501.

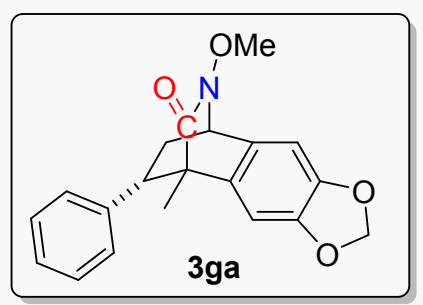

11-Methoxy-8-methyl-7-phenyl-5,6,7,8-tetrahydro-5,8-( epiminomethano)naphtho[2,3-d][1,3]dioxol-10-one

(3ga): the title compound was prepared according to general procedure and purified by column chromatography (petroleum ether/ethyl acetate $=5 / 1$ to $3 / 1$ ) to give a white solid (117 mg, 70\% yield, m.p. 126-165 $\left.{ }^{\circ} \mathrm{C}\right) .{ }^{1} \mathbf{H}$ NMR $\left(400 \mathrm{MHz}, \mathrm{CDCl}_{3}\right) \delta 1.18(\mathrm{~s}$, $3 \mathrm{H}), 1.69-1.74(\mathrm{~m}, 1 \mathrm{H}), 2.83-2.89(\mathrm{~m}, 1 \mathrm{H}), 3.04\left(\mathrm{dd}, J_{l}=10.0 \mathrm{~Hz}, J_{2}=4.8 \mathrm{~Hz}, 1 \mathrm{H}\right)$, $3.74(\mathrm{~s}, 3 \mathrm{H}), 4.76\left(\mathrm{dd}, J_{1}=3.6 \mathrm{~Hz}, J_{2}=2.0 \mathrm{~Hz}, 1 \mathrm{H}\right), 5.89(\mathrm{~d}, J=1.2 \mathrm{~Hz}, 1 \mathrm{H}), 5.95(\mathrm{~d}$, $J=1.2 \mathrm{~Hz}, 1 \mathrm{H}), 6.32-6.34(\mathrm{~m}, 2 \mathrm{H}), 6.40(\mathrm{~s}, 1 \mathrm{H}), 6.83(\mathrm{~s}, 1 \mathrm{H}), 7.02-7.11(\mathrm{~m}, 3 \mathrm{H}) ;{ }^{13} \mathrm{C}$ NMR $\left(100 \mathrm{MHz}, \mathrm{CDCl}_{3}\right) \delta 14.6,38.3,46.0,52.4,60.6,63.4,101.2,103.3,106.6$, 127.0, 128.0, 128.5, 131.4, 133.7, 140.6, 146.6, 147.3, 173.7; HRMS (ESI) $m / z$ : $[\mathrm{M}+\mathrm{Na}]^{+}$Calcd for $\mathrm{C}_{20} \mathrm{H}_{19} \mathrm{NNaO}_{4} 360.1206$, found 360.1209.

4-Methyl-3,10-diphenyl-1,2,3,4-tetrahydro-1,4-(epiminomethano)naphthalen-9-o 


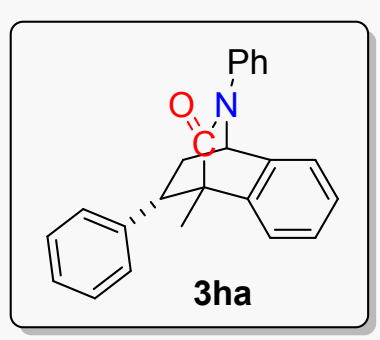

ne (3ha) : the title compound was prepared according to general procedure and purified by column chromatography (petroleum ether/ethyl acetate $=5 / 1$ to $3 / 1$ ) to give a white solid $\left(92.8 \mathrm{mg}, 61 \%\right.$ yield, m.p. $\left.150-152{ }^{\circ} \mathrm{C}\right)$. ${ }^{1} \mathbf{H}$ NMR $\left(400 \mathrm{MHz}, \mathrm{CDCl}_{3}\right) \delta 1.30(\mathrm{~s}, 3 \mathrm{H}), 1.79-1.84(\mathrm{~m}$, $1 \mathrm{H}), 2.91-2.98(\mathrm{~m}, 1 \mathrm{H}), 3.23\left(\mathrm{dd}, J_{1}=10.0 \mathrm{~Hz}, J_{2}=5.2 \mathrm{~Hz}, 1 \mathrm{H}\right), 5.14\left(\mathrm{dd}, J_{1}=4.0 \mathrm{~Hz}\right.$, $\left.J_{2}=2.0 \mathrm{~Hz}, 1 \mathrm{H}\right), 6.28(\mathrm{~d}, J=7.6 \mathrm{~Hz}, 2 \mathrm{H}), 6.97-7.16(\mathrm{~m}, 5 \mathrm{H}), 7.22-7.35(\mathrm{~m}, 7 \mathrm{H}) ;{ }^{13} \mathrm{C}$ NMR $\left(100 \mathrm{MHz}, \mathrm{CDCl}_{3}\right) \delta 14.6,38.8,45.7,52.0,60.7,121.3,123.8,125.1,125.8$, $126.9,127.1,127.8,127.9,128.5,128.9,138.3,140.4,140.9,141.1,173.7$; HRMS (ESI) $m / z$ : $[\mathrm{M}+\mathrm{Na}]^{+}$Calcd for $\mathrm{C}_{24} \mathrm{H}_{21} \mathrm{NNaO} 362.1515$, found 362.1512 .

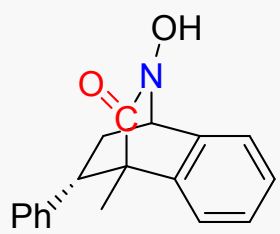

3 ia, $0 \%$ yield

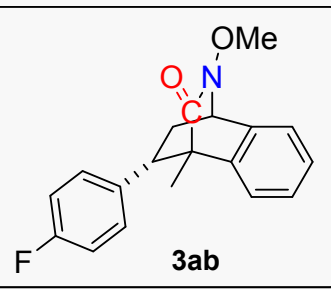

$\mathrm{Ph}$

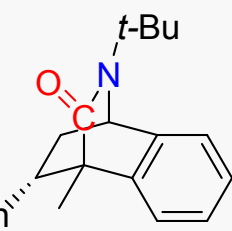

$3 \mathbf{j a}, 0 \%$ yield

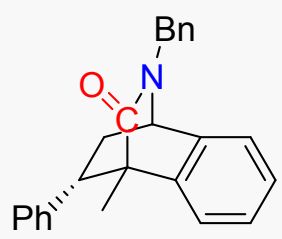

$3 \mathbf{k a}, 0 \%$ yield

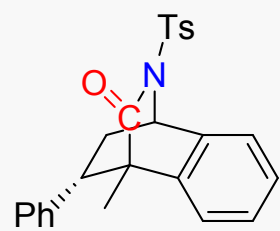

$31 a, 0 \%$ yield

3-(4-Fluorophenyl)-10-methoxy-4-methyl-1,2,3,4-tetrahyd ro-1,4-(epiminomethano)naphthalen-9-one (3ab): the title compound was prepared according to general procedure and purified by column chromatography (petroleum ether/ethyl acetate $=5 / 1$ to $3 / 1)$ to give a white solid $(107 \mathrm{mg}, 69 \%$ yield, m.p. 205-209 $\left.{ }^{\circ} \mathrm{C}\right) .{ }^{1} \mathbf{H}$ NMR $\left(400 \mathrm{MHz}, \mathrm{CDCl}_{3}\right) \delta 1.30$ (s, 3H), 1.72-1.77 (m, 1H), 2.93-3.00 (m, 1H), $3.17\left(\mathrm{dd}, J_{1}=10.0 \mathrm{~Hz}, J_{2}=4.4 \mathrm{~Hz}, 1 \mathrm{H}\right), 3.82(\mathrm{~s}, 3 \mathrm{H}), 4.95-4.96(\mathrm{~m}$, 1H), $6.23(\mathrm{~s}, 2 \mathrm{H}), 6.75$ (t, $J=8.4 \mathrm{~Hz}, 2 \mathrm{H}), 6.94(\mathrm{~d}, J=7.4 \mathrm{~Hz}, 1 \mathrm{H}), 7.27-7.31(\mathrm{~m}, 1 \mathrm{H})$, 7.34-7.39 (m, 2H); ${ }^{13} \mathbf{C}$ NMR $\left(100 \mathrm{MHz}, \mathrm{CDCl}_{3}\right) \delta 14.2,38.4,45.6,52.7,60.7,63.5$, $114.8(\mathrm{~d}, J=20 \mathrm{~Hz}), 121.6,125.1,127.5,128.1,129.9$ (d, $J=10 \mathrm{~Hz}), 136.4(\mathrm{~d}, J=10$ $\mathrm{Hz}), 137.6,140.0,160.7(\mathrm{~d}, J=244 \mathrm{~Hz}), 173.2 ;{ }^{19} \mathbf{F}$ NMR $\left(376 \mathrm{MHz}, \mathrm{CDCl}_{3}\right) \delta$ -115.9; HRMS (ESI) $m / z$ : $[\mathrm{M}+\mathrm{H}]^{+}$Calcd for $\mathrm{C}_{19} \mathrm{H}_{19} \mathrm{NO}_{2} \mathrm{~F} 312.1400$, found 312.1400. 


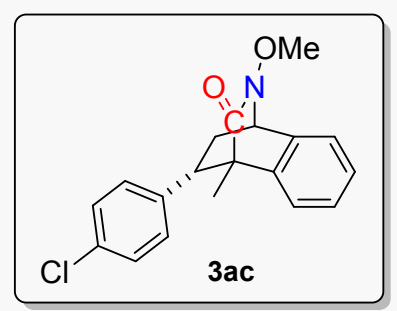

3-(4-Chlorophenyl)-10-methoxy-4-methyl-1,2,3,4-tetrahy dro-1,4-(epiminomethano)naphthalen-9-one (3ac): the title compound was prepared according to general procedure and purified by column chromatography (petroleum ether/ethyl acetate $=5 / 1$ to $3 / 1)$ to give a white solid (121 mg, 74\% yield, m.p. 206-207 $\left.{ }^{\circ} \mathrm{C}\right) .{ }^{1} \mathbf{H}$ NMR (400 MHz, $\left.\mathrm{CDCl}_{3}\right) \delta 1.30(\mathrm{~s}, 3 \mathrm{H})$, 1.70-1.75 (m, 1H), 2.92-2.99 (m, 1H), $3.15\left(\mathrm{dd}, J_{1}=10.0 \mathrm{~Hz}, J_{2}=4.8 \mathrm{~Hz}, 1 \mathrm{H}\right), 3.81(\mathrm{~s}$, $3 \mathrm{H}), 4.95\left(\mathrm{dd}, J_{1}=4.0 \mathrm{~Hz}, J_{2}=2.0 \mathrm{~Hz}, 1 \mathrm{H}\right), 6.18(\mathrm{~d}, J=7.6 \mathrm{~Hz}, 2 \mathrm{H}), 6.94(\mathrm{~d}, J=7.6$ $\mathrm{Hz}, 1 \mathrm{H}), 7.01-7.03(\mathrm{~m}, 2 \mathrm{H}), 7.26-7.39(\mathrm{~m}, 3 \mathrm{H}) ;{ }^{13} \mathbf{C ~ N M R}\left(100 \mathrm{MHz}, \mathrm{CDCl}_{3}\right) \delta 14.1$, $38.1,45.6,52.5,60.5,63.4,121.5,124.9,127.5,128.0,128.1,130.0,132.8,137.3$, 139.1, 139.9, 173.0; HRMS (ESI) $m / z$ : $[\mathrm{M}+\mathrm{Na}]^{+}$Calcd for $\mathrm{C}_{19} \mathrm{H}_{18} \mathrm{NNaO}_{2} \mathrm{Cl} 350.0918$, found:350.0919.

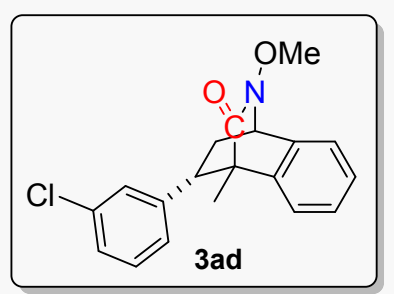

3-(3-Chlorophenyl)-10-methoxy-4-methyl-1,2,3,4-tetrahy dro-1,4-(epiminomethano)naphthalen-9-one (3ad): the title compound was prepared according to general procedure and purified by column chromatography (petroleum ether/ethyl acetate $=5 / 1$ to $3 / 1)$ to give a white solid $(121$ mg, 74\% yield, m.p. 167-172 $\left.{ }^{\circ} \mathrm{C}\right) .{ }^{1} \mathbf{H}$ NMR (400 MHz, $\left.\mathrm{CDCl}_{3}\right) \delta 1.32(\mathrm{~s}, 3 \mathrm{H})$, 1.72-1.77 (m, 1H), 2.93-2.99 (m, 1H), $3.14\left(\mathrm{dd}, J_{1}=10.4 \mathrm{~Hz}, J_{2}=4.8 \mathrm{~Hz}, 1 \mathrm{H}\right), 3.81(\mathrm{~s}$, $3 \mathrm{H}), 4.95\left(\mathrm{dd}, J_{1}=4.0 \mathrm{~Hz}, J_{2}=1.6 \mathrm{~Hz}, 1 \mathrm{H}\right), 6.17-6.20(\mathrm{~m}, 2 \mathrm{H}), 6.96-7.01(\mathrm{~m}, 2 \mathrm{H})$, $7.09\left(\mathrm{dd}, J_{1}=6.0 \mathrm{~Hz}, J_{2}=2.0 \mathrm{~Hz}, 1 \mathrm{H}\right), 7.28-7.41(\mathrm{~m}, 3 \mathrm{H}) ;{ }^{13} \mathbf{C}$ NMR $(100 \mathrm{MHz}$, $\left.\mathrm{CDCl}_{3}\right) \delta 14.1,38.1,45.9,52.5,60.5,63.4,121.5,124.9,126.7,127.1,127.5,128.0$, 128.4, 129.1, 133.7, 137.3, 139.8, 142.7, 172.9; HRMS (ESI) $m / z:[\mathrm{M}+\mathrm{Na}]^{+}$Calcd for $\mathrm{C}_{19} \mathrm{H}_{18} \mathrm{ClNNaO}_{2}$ 350.0918, found 350.0916.

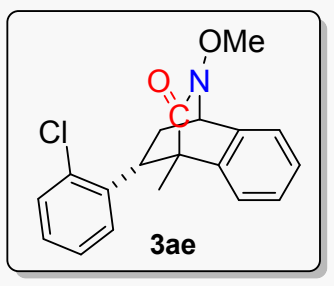

\section{3-(2-Chlorophenyl)-10-methoxy-4-methyl-1,2,3,4-tetrahydro} -1,4-(epiminomethano)naphthalen-9-one (3ae): the title compound was prepared according to general procedure and purified by column chromatography (petroleum ether/ethyl acetate $=5 / 1$ to $3 / 1)$ to give a white solid $(18.0 \mathrm{mg}, 11 \%$ yield, m.p. $\left.207-210^{\circ} \mathrm{C}\right) .{ }^{1} \mathbf{H}$ NMR $\left(400 \mathrm{MHz}, \mathrm{CDCl}_{3}\right) \delta 1.40(\mathrm{~s}, 3 \mathrm{H}), 1.64-1.69(\mathrm{~m}, 1 \mathrm{H})$, 
2.96-3.03 (m, 1H), $3.83(\mathrm{~s}, 3 \mathrm{H}), 4.06\left(\mathrm{dd}, J_{1}=10.4 \mathrm{~Hz}, J_{2}=5.2 \mathrm{~Hz}, 1 \mathrm{H}\right), 4.95\left(\mathrm{dd}, J_{1}\right.$ $\left.=3.6 \mathrm{~Hz}, J_{2}=1.6 \mathrm{~Hz}, 1 \mathrm{H}\right), 5.40\left(\mathrm{dd}, J_{1}=8.0 \mathrm{~Hz}, J_{2}=1.6 \mathrm{~Hz}, 1 \mathrm{H}\right), 6.77-6.81(\mathrm{~m}, 1 \mathrm{H})$, 6.99-7.06 (m, 2H), 7.27-7.32 (m, 2H), 7.34-7.40 (m, 2H); ${ }^{13} \mathbf{C}$ NMR (100 MHz, $\left.\mathrm{CDCl}_{3}\right) \delta 12.9,38.5,39.6,53.1,60.6,63.5,121.6,125.2,126.6,127.6,127.9,128.1$, 128.1, 129.3, 135.4, 137.6, 138.5, 140.2, 173.1; HRMS (ESI) $m / z:[\mathrm{M}+\mathrm{H}]^{+}$Calcd for $\mathrm{C}_{19} \mathrm{H}_{19} \mathrm{NO}_{2} \mathrm{Cl} \quad 328.1104$, found 328.1104.

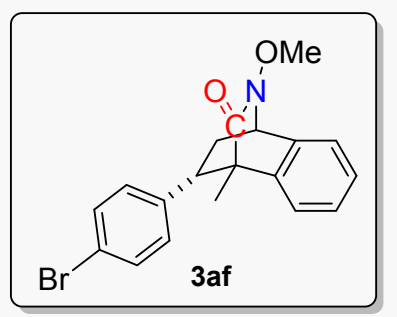

\section{3-(4-Bromophenyl)-10-methoxy-4-methyl-1,2,3,4-tetrahy} dro-1,4-(epiminomethano)naphthalen-9-one (3af): the title compound was prepared according to general procedure and purified by column chromatography (petroleum ether/ethyl acetate $=5 / 1$ to $3 / 1)$ to give a white solid $(128 \mathrm{mg}$, $69 \%$ yield, m.p. $\left.198-200{ }^{\circ} \mathrm{C}\right) .{ }^{1} \mathbf{H}$ NMR $\left(400 \mathrm{MHz}, \mathrm{CDCl}_{3}\right) \delta 1.30(\mathrm{~s}, 3 \mathrm{H}), 1.70-1.75$ $(\mathrm{m}, 1 \mathrm{H}), 2.93-3.00(\mathrm{~m}, 1 \mathrm{H}), 3.14\left(\mathrm{dd}, J_{l}=10.0 \mathrm{~Hz}, J_{2}=4.8 \mathrm{~Hz}, 1 \mathrm{H}\right), 3.81(\mathrm{~s}, 3 \mathrm{H})$, $4.94\left(\mathrm{dd}, J_{1}=3.6 \mathrm{~Hz}, J_{2}=1.6 \mathrm{~Hz}, 1 \mathrm{H}\right), 6.13(\mathrm{~d}, J=8.0 \mathrm{~Hz}, 2 \mathrm{H}), 6.95(\mathrm{~d}, J=7.5 \mathrm{~Hz}$, $1 \mathrm{H}), 7.17\left(\mathrm{dd}, J_{l}=7.4 \mathrm{~Hz}, J_{2}=1.3 \mathrm{~Hz}, 2 \mathrm{H}\right), 7.27-7.30(\mathrm{~m}, 1 \mathrm{H}), 7.34-7.39(\mathrm{~m}, 2 \mathrm{H})$; ${ }^{13} \mathrm{C}$ NMR $\left(100 \mathrm{MHz}, \mathrm{CDCl}_{3}\right) \delta$ 14.2, 38.2, 45.8, 52.6, 60.7, 63.5, 121.1, 121.6, 125.1, 127.6, 128.1, 130.2, 131.2, 137.5, 139.7, 140.0, 173.1; HRMS (ESI) $m / z:[\mathrm{M}+\mathrm{H}]^{+}$ Calcd for $\mathrm{C}_{19} \mathrm{H}_{19} \mathrm{NO}_{2} \mathrm{Br} 372.0599$, found 372.0591.

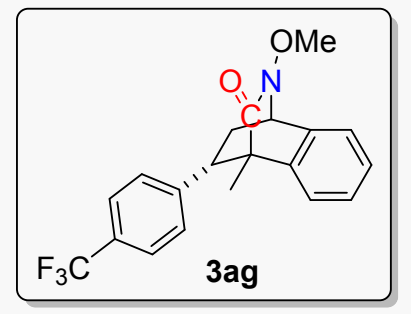

\section{3-(4-Trifluoromethyl)-10-methoxy-4-methyl-1,2,3,4-tetra} hydro-1,4-(epiminomethano)naphthalen-9-one (3ag): the title compound was prepared according to general procedure and purified by column chromatography (petroleum ether/ethyl acetate $=5 / 1$ to $3 / 1)$ to give a white solid $(57.8$ mg, 32\% yield, m.p. 182-184 $\left.{ }^{\circ} \mathrm{C}\right) .{ }^{1} \mathbf{H}$ NMR (400 MHz, $\left.\mathrm{CDCl}_{3}\right) \delta 1.31$ (s, 3H), $1.75-1.80(\mathrm{~m}, 1 \mathrm{H}), 2.96-3.03(\mathrm{~m}, 1 \mathrm{H}), 3.24\left(\mathrm{dd}, J_{1}=10.0 \mathrm{~Hz}, J_{2}=4.8 \mathrm{~Hz}, 1 \mathrm{H}\right), 3.83(\mathrm{~s}$, $3 \mathrm{H}), 4.98\left(\mathrm{dd}, J_{l}=3.6 \mathrm{~Hz}, J_{2}=1.6 \mathrm{~Hz}, 1 \mathrm{H}\right), 6.38(\mathrm{~d}, J=8.0 \mathrm{~Hz}, 2 \mathrm{H}), 6.96(\mathrm{~d}, J=7.6$ $\mathrm{Hz}, 1 \mathrm{H}), 7.29-7.33$ (m, 3H), 7.36-7.42 (m, 2H); $\left.{ }^{13} \mathbf{C ~ N M R ~ ( 1 0 0 ~ M H z , ~} \mathrm{CDCl}_{3}\right) \delta 14.1$, 38.1, 46.0, 52.4, 60.5, 63.4, 121.5, 122.7 (d, $J=270 \mathrm{~Hz}$ ), 124.8 (q, $J=4 \mathrm{~Hz}), 125.0$, 127.6, 128.1, 128.7, 129.1 (d, $J=32 \mathrm{~Hz}), 137.2,139.9,144.7,172.8 ;{ }^{19} \mathbf{F}$ NMR (376 $\mathrm{MHz}, \mathrm{CDCl}_{3}$ ) $\delta$-62.5; HRMS (ESI) $m / z$ : $[\mathrm{M}+\mathrm{H}]^{+}$Calcd for $\mathrm{C}_{20} \mathrm{H}_{19} \mathrm{NO}_{2} \mathrm{~F}_{3} 362.1368$, 
found 362.1368 .

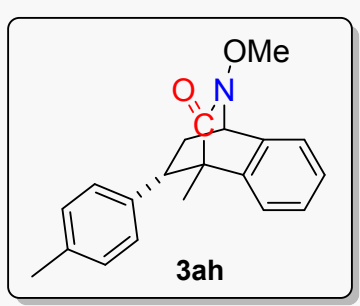

10-Methoxy-4-methyl-3-(p-tolyl)-1,2,3,4-tetrahydro-1,4-(e piminomethano)naphthalen-9-one (3ah): the title compound was prepared according to general procedure and purified by column chromatography (petroleum ether/ethyl acetate $=5 / 1$ to $3 / 1)$ to give a white solid $(121 \mathrm{mg}, 79 \%$ yield, m.p. $\left.152-155{ }^{\circ} \mathrm{C}\right) .{ }^{1} \mathbf{H}$ NMR (400 MHz, $\left.\mathrm{CDCl}_{3}\right) \delta 1.31(\mathrm{~s}, 3 \mathrm{H}), 1.74-1.79(\mathrm{~m}$, 1H), $2.23(\mathrm{~s}, 3 \mathrm{H}), 2.91-2.98(\mathrm{~m}, 1 \mathrm{H}), 3.14\left(\mathrm{dd}, J_{1}=10.4 \mathrm{~Hz}, J_{2}=4.8 \mathrm{~Hz}, 1 \mathrm{H}\right), 3.81(\mathrm{~s}$, $3 \mathrm{H}), 4.94\left(\mathrm{dd}, J_{1}=3.6 \mathrm{~Hz}, J_{2}=1.6 \mathrm{~Hz}, 1 \mathrm{H}\right), 6.17(\mathrm{~d}, J=7.6 \mathrm{~Hz}, 2 \mathrm{H}), 6.86(\mathrm{~d}, J=8.4$ Hz, 2H), $6.95(\mathrm{~d}, J=7.6 \mathrm{~Hz}, 1 \mathrm{H}), 7.25-7.29(\mathrm{~m}, 1 \mathrm{H}), 7.33-7.39(\mathrm{~m}, 2 \mathrm{H}) ;{ }^{13} \mathbf{C} \mathbf{N M R}$ $\left(100 \mathrm{MHz}, \mathrm{CDCl}_{3}\right) \delta 14.2,21.0,38.3,45.8,52.7,60.7,63.4,121.5,125.0,127.3$, 127.9, 128.4, 128.7, 136.6, 137.6, 137.8, 140.1, 173.5; HRMS (ESI) $m / z:[\mathrm{M}+\mathrm{H}]^{+}$ Calcd for $\mathrm{C}_{20} \mathrm{H}_{22} \mathrm{NO}_{2}$ 308.1651, found 308.1649.

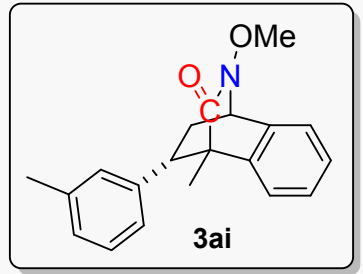

10-Methoxy-4-methyl-3-(m-tolyl)-1,2,3,4-tetrahydro-1,4-(e piminomethano)naphthalen-9-one (3ai): the title compound was prepared according to general procedure and purified by column chromatography (petroleum ether/ethyl acetate $=5 / 1$ to $3 / 1)$ to give a white solid $(117 \mathrm{mg}, 76 \%$ yield, m.p. $\left.182-186{ }^{\circ} \mathrm{C}\right) .{ }^{1} \mathbf{H}$ NMR $\left(400 \mathrm{MHz}, \mathrm{CDCl}_{3}\right) \delta 1.31(\mathrm{~s}, 3 \mathrm{H}), 1.76-1.81(\mathrm{~m}, 1 \mathrm{H})$, $2.13(\mathrm{~s}, 3 \mathrm{H}), 2.91-2.98(\mathrm{~m}, 1 \mathrm{H}), 3.13\left(\mathrm{dd}, J_{1}=10.4 \mathrm{~Hz}, J_{2}=4.8 \mathrm{~Hz}, 1 \mathrm{H}\right), 3.82(\mathrm{~s}, 3 \mathrm{H})$, $4.94\left(\mathrm{dd}, J_{1}=3.6 \mathrm{~Hz}, J_{2}=1.6 \mathrm{~Hz}, 1 \mathrm{H}\right), 6.05-6.06(\mathrm{~m}, 2 \mathrm{H}), 6.93-6.97(\mathrm{~m}, 3 \mathrm{H})$, 7.28-7.30 (m, 1H), 7.33-7.39 (m, 2H); $\left.{ }^{13} \mathbf{C ~ N M R ~ ( 1 0 0 ~ M H z , ~} \mathrm{CDCl}_{3}\right) \delta$ 14.1, 21.3, $38.2,46.1,52.6,60.7,63.3,121.4,125.0,125.4,127.2,127.6,127.7,129.4,137.4$, 137.8, 140.0, 140.5, 173.4; HRMS (ESI) $m / z$ : $[\mathrm{M}+\mathrm{H}]^{+}$Calcd for $\mathrm{C}_{20} \mathrm{H}_{22} \mathrm{NO}_{2}$ 308.1651, found 308.1648 .

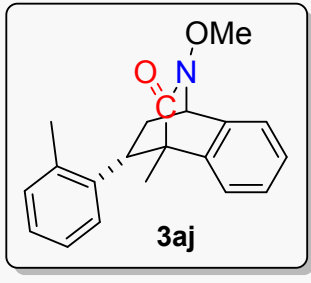

10-Methoxy-4-methyl-3-(o-tolyl)-1,2,3,4-tetrahydro-1,4-(epim inomethano)naphthalen-9-one (3aj): the title compound was prepared according to general procedure and purified by column chromatography (petroleum ether/ethyl acetate $=5 / 1$ to $3 / 1$ ) to give a white solid (84.4 mg, 55\% yield, m.p. $\left.168-171{ }^{\circ} \mathrm{C}\right) .{ }^{1} \mathbf{H}$ NMR (400 MHz, 
$\left.\mathrm{CDCl}_{3}\right) \delta 1.34(\mathrm{~s}, 3 \mathrm{H}), 1.66-1.71(\mathrm{~m}, 1 \mathrm{H}), 2.39(\mathrm{~s}, 3 \mathrm{H}), 2.93-3.00(\mathrm{~m}, 1 \mathrm{H}), 3.62(\mathrm{dd}$, $\left.J_{1}=9.6 \mathrm{~Hz}, J_{2}=5.6 \mathrm{~Hz}, 1 \mathrm{H}\right), 3.82(\mathrm{~s}, 3 \mathrm{H}), 4.94-4.95(\mathrm{~m}, 1 \mathrm{H}), 5.36(\mathrm{~d}, J=8.0 \mathrm{~Hz}, 1 \mathrm{H})$, $6.73(\mathrm{t}, J=7.8 \mathrm{~Hz}, 1 \mathrm{H}), 6.97-7.10(\mathrm{~m}, 3 \mathrm{H}), 7.28-7.39$ (m, 3H); ${ }^{13} \mathbf{C}$ NMR (100 MHz, $\left.\mathrm{CDCl}_{3}\right) \delta 13.0,20.7,38.7,39.3,53.2,60.7,63.4,121.4,125.2,125.9,126.4,126.5$, $127.3,127.9,130.1,137.0,137.8,139.3,140.2,173.6$; HRMS (ESI) $m / z:[\mathrm{M}+\mathrm{H}]^{+}$ Calcd for $\mathrm{C}_{20} \mathrm{H}_{22} \mathrm{NO}_{2} \quad 308.1645$, found 308.1646 .

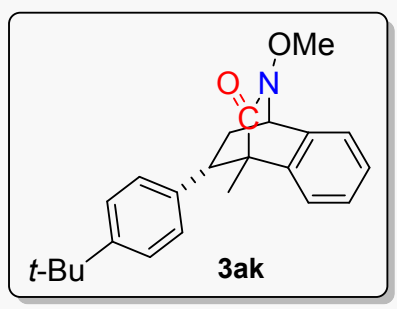

\section{3-(4-(tert-Butyl)phenyl)-10-methoxy-4-methyl-1,2,3,4-tetr} ahydro-1,4-(epiminomethano)naphthalen-9-one (3ak): the title compound was prepared according to general procedure and purified by column chromatography (petroleum ether/ethyl acetate $=5 / 1$ to $3 / 1$ ) to give a white solid (55.8 mg, 32\% yield, m.p. $\left.125-127{ }^{\circ} \mathrm{C}\right) .{ }^{1} \mathbf{H}$ NMR $\left(400 \mathrm{MHz}, \mathrm{CDCl}_{3}\right) \delta 1.23(\mathrm{~s}$, $9 \mathrm{H}), 1.32(\mathrm{~s}, 3 \mathrm{H}), 1.75-1.80(\mathrm{~m}, 1 \mathrm{H}), 2.91-2.98(\mathrm{~m}, 1 \mathrm{H}), 3.15\left(\mathrm{dd}, J_{1}=10.4 \mathrm{~Hz}, J_{2}=\right.$ $5.2 \mathrm{~Hz}, 1 \mathrm{H}), 3.82(\mathrm{~s}, 3 \mathrm{H}), 4.93\left(\mathrm{dd}, J_{1}=3.6 \mathrm{~Hz}, J_{2}=1.2 \mathrm{~Hz}, 1 \mathrm{H}\right), 6.22(\mathrm{~d}, J=7.6 \mathrm{~Hz}$, 2H), $7.00(\mathrm{~d}, J=7.2 \mathrm{~Hz}, 1 \mathrm{H}), 7.07\left(\mathrm{dd}, J_{1}=7.2 \mathrm{~Hz}, J_{2}=1.2 \mathrm{~Hz}, 2 \mathrm{H}\right), 7.27-7.31(\mathrm{~m}$, 1H), 7.33-7.38 (m, 2H); ${ }^{13} \mathbf{C}$ NMR (100 MHz, $\left.\mathrm{CDCl}_{3}\right) \delta 14.3,31.4,34.5,38.4,45.7$, $52.7,60.8,63.4,121.4,124.9,125.1,127.3,127.9,128.2,137.6,137.9,140.2,149.8$, 173.5; HRMS (ESI) $m / z$ : $[\mathrm{M}+\mathrm{H}]^{+}$Calcd for $\mathrm{C}_{23} \mathrm{H}_{28} \mathrm{NO}_{2}$ 350.2120, found 350.2119.

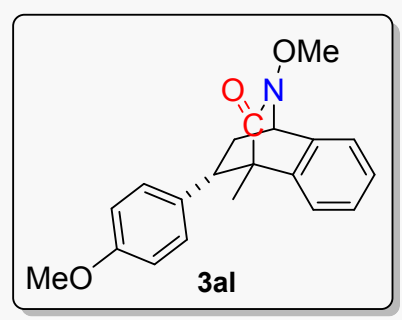

10-Methoxy-3-(4-methoxyphenyl)-4-methyl-1,2,3,4-tetra hydro-1,4-(epiminomethano)naphthalen-9-one (3al): the title compound was prepared according to general procedure and purified by column chromatography (petroleum ether/ethyl acetate $=5 / 1$ to $3 / 1$ ) to give a white solid (131 mg, 81\% yield, m.p. 165-170 $\left.{ }^{\circ} \mathrm{C}\right) .{ }^{1} \mathbf{H}$ NMR $\left(400 \mathrm{MHz}, \mathrm{CDCl}_{3}\right) \delta 1.31(\mathrm{~s}$, $3 \mathrm{H}), 1.72-1.77(\mathrm{~m}, 1 \mathrm{H}), 2.91-2.98(\mathrm{~m}, 1 \mathrm{H}), 3.13\left(\mathrm{dd}, J_{1}=10.4 \mathrm{~Hz}, J_{2}=4.8 \mathrm{~Hz}, 1 \mathrm{H}\right)$, $3.71(\mathrm{~s}, 3 \mathrm{H}), 3.81(\mathrm{~s}, 3 \mathrm{H}), 4.93\left(\mathrm{dd}, J_{1}=4.0 \mathrm{~Hz}, J_{2}=2.0 \mathrm{~Hz}, 1 \mathrm{H}\right), 6.19(\mathrm{~d}, J=8.0 \mathrm{~Hz}$, 2H), 6.59 (d, $J=9.2 \mathrm{~Hz}, 2 \mathrm{H}), 6.95$ (d, $J=7.2 \mathrm{~Hz}, 1 \mathrm{H}), 7.25-7.30$ (m, 1H), 7.32-7.39 $(\mathrm{m}, 2 \mathrm{H}) ;{ }^{13} \mathrm{C}$ NMR $\left(100 \mathrm{MHz}, \mathrm{CDCl}_{3}\right) \delta 14.2,38.3,45.5,52.8,55.2,60.7,63.4,113.3$, 121.4, 125.0, 127.3, 127.9, 129.5, 132.6, 137.8, 140.1, 158.6, 173.5; HRMS (ESI) $m / z$ : $[\mathrm{M}+\mathrm{H}]^{+}$Calcd for $\mathrm{C}_{20} \mathrm{H}_{22} \mathrm{NO}_{3} 324.1600$, found 324.1598 . 


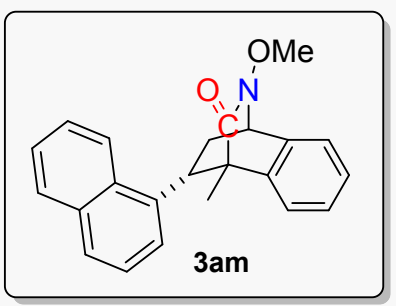

10-Methoxy-4-methyl-3-(naphthalen-1-yl)-1,2,3,4-tetrah ydro-1,4-(epiminomethano)naphthalen-9-one (3am): the title compound was prepared according to general procedure and purified by column chromatography (petroleum ether/ethyl acetate $=5 / 1$ to $3 / 1$ ) to give a yellow solid (75.5 mg, 44\% yield, m.p. $\left.151-155{ }^{\circ} \mathrm{C}\right) .{ }^{1} \mathbf{H}$ NMR $\left(400 \mathrm{MHz}, \mathrm{CDCl}_{3}\right) \delta 1.34(\mathrm{~s}$, $3 \mathrm{H}), 1.88-1.93(\mathrm{~m}, 1 \mathrm{H}), 2.99-3.06(\mathrm{~m}, 1 \mathrm{H}), 3.15\left(\mathrm{dd}, J_{l}=10.0 \mathrm{~Hz}, J_{2}=4.8 \mathrm{~Hz}, 1 \mathrm{H}\right)$, $3.84(\mathrm{~s}, 3 \mathrm{H}), 4.99\left(\mathrm{dd}, J_{1}=3.6 \mathrm{~Hz}, J_{2}=1.6 \mathrm{~Hz}, 1 \mathrm{H}\right), 6.24-6.25(\mathrm{~m}, 1 \mathrm{H}), 6.85-6.86(\mathrm{~m}$, 1H), $6.93(\mathrm{~d}, J=7.2 \mathrm{~Hz}, 1 \mathrm{H}), 7.26-7.30(\mathrm{~m}, 1 \mathrm{H}), 7.37-7.44(\mathrm{~m}, 4 \mathrm{H}), 7.50$ (d, $J=8.4$ $\mathrm{Hz}, 1 \mathrm{H}), 7.53-7.56(\mathrm{~m}, 1 \mathrm{H}), 7.69-7.73(\mathrm{~m}, 1 \mathrm{H}) ;{ }^{13} \mathbf{C ~ N M R}\left(100 \mathrm{MHz}, \mathrm{CDCl}_{3}\right) \delta 14.3$, $38.3,46.5,52.9,60.8,63.5,121.6,125.1,125.8,126.1,126.3,127.5,127.5,127.6$, 127.7, 127.7, 128.0, 132.5, 133.0, 137.9, 138.2, 140.1, 173.3; HRMS (ESI) $m / z$ : $[\mathrm{M}+\mathrm{H}]^{+}$Calcd for $\mathrm{C}_{23} \mathrm{H}_{22} \mathrm{NO}_{2}$ 344.1645, found 344.1646.

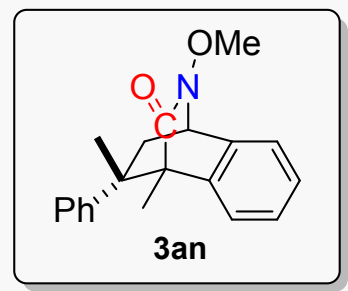

10-Methoxy-3,4-dimethyl-3-phenyl-1,2,3,4-tetrahydro-1,4-(e piminomethano)naphthalen-9-one (3an): the title compound was prepared according to general procedure and purified by column chromatography (petroleum ether/ethyl acetate $=5 / 1$ to 3/1) to give a white solid $(49.1 \mathrm{mg}, 32 \%$ yield, m.p. 125-130 $\left.{ }^{\circ} \mathrm{C}\right) .{ }^{1} \mathbf{H}$ NMR $\left(400 \mathrm{MHz}, \mathrm{CDCl}_{3}\right) \delta 1.26(\mathrm{~s}, 3 \mathrm{H}), 1.67(\mathrm{~s}, 3 \mathrm{H}), 2.33\left(\mathrm{dd}, J_{l}=\right.$ $\left.13.6 \mathrm{~Hz}, J_{2}=2.0 \mathrm{~Hz}, 1 \mathrm{H}\right), 2.51\left(\mathrm{dd}, J_{1}=13.6 \mathrm{~Hz}, J_{2}=3.6 \mathrm{~Hz}, 1 \mathrm{H}\right), 3.84(\mathrm{~s}, 3 \mathrm{H}), 4.94$ $\left(\mathrm{dd}, J_{1}=3.6 \mathrm{~Hz}, J_{2}=2.0 \mathrm{~Hz}, 1 \mathrm{H}\right), 6.35-6.38(\mathrm{~m}, 2 \mathrm{H}), 6.78(\mathrm{~d}, J=7.6 \mathrm{~Hz}, 1 \mathrm{H})$, 6.99-7.03 (m, 2H), 7.06-7.10 (m, 1H), $7.18\left(\mathrm{dt}, J_{1}=7.6 \mathrm{~Hz}, J_{2}=1.6 \mathrm{~Hz}, 1 \mathrm{H}\right)$, 7.29-7.36 (m, 2H); ${ }^{13} \mathbf{C}$ NMR (100 MHz, $\left.\mathrm{CDCl}_{3}\right) \delta 10.9,26.0,43.7,46.1,56.7,60.5$, $63.3,121.1,125.3,126.3,127.1,127.2,127.3,127.7,139.1,140.2,143.8,172.5$; HRMS (ESI) $m / z$ : $[\mathrm{M}+\mathrm{H}]^{+}$Calcd for $\mathrm{C}_{20} \mathrm{H}_{22} \mathrm{NO}_{2}$ 308.1651, found 308.1647.

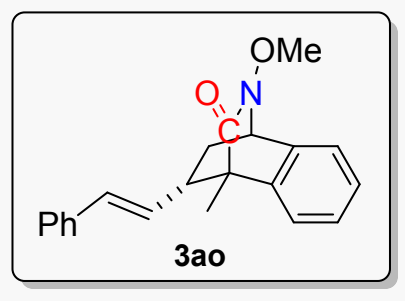

10-Methoxy-4-methyl-3-styryl-1,2,3,4-tetrahydro-1,4-(ep iminomethano)naphthalen-9-one (3ao): the title compound was prepared according to general procedure and purified by column chromatography (petroleum ether/ethyl acetate $=5 / 1$ to $3 / 1)$ to give a white solid $\left(81.3 \mathrm{mg}, 51 \%\right.$ yield, m.p. $\left.116-120^{\circ} \mathrm{C}\right) .{ }^{1} \mathbf{H}$ 
NMR $\left(400 \mathrm{MHz}, \mathrm{CDCl}_{3}\right) \delta 1.50-1.53(\mathrm{~m}, 1 \mathrm{H}), 1.62(\mathrm{~s}, 3 \mathrm{H}), 2.75-2.82(\mathrm{~m}, 2 \mathrm{H}), 3.81$ (s, 3H), 4.86-4.87 (m, 1H), 5.15-5.21 (m, 1H), 6.34 (s, J=15.6 Hz, 1H), 7.18-7.28 (m, 6H), 7.32-7.38 (m, 3H); ${ }^{13} \mathrm{C}$ NMR (100 MHz, $\left.\mathrm{CDCl}_{3}\right) \delta 14.1,38.1,44.4,51.6,60.3$, 63.3, 121.6, 123.9, 126.2, 127.1, 127.5, 127.9, 128.5, 129.5, 132.3, 136.7, 138.0, 139.5, 173.1; HRMS (ESI) $m / z$ : $[\mathrm{M}+\mathrm{Na}]^{+}$Calcd for $\mathrm{C}_{21} \mathrm{H}_{21} \mathrm{NNaO}_{2} 342.1464$, found 342.1462 .

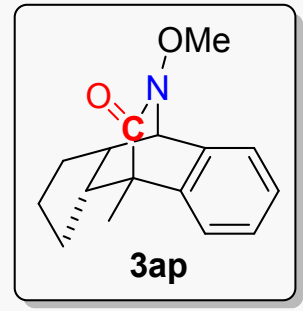

\section{1-Methoxy-9-methyl-2,3,3a,4,9,9a-hexahydro-1H-4,9-(epimi} nomethano)cyclopenta[b]naphthalen-10-one (3ap): The title compound was prepared according to general procedure and purified by column chromatography (petroleum ether/ethyl acetate $=5 / 1$ to $3 / 1)$ to give a white solid $(87.4 \mathrm{mg}, 68 \%$ yield, m.p. $\left.118-120^{\circ} \mathrm{C}\right) .{ }^{1} \mathbf{H}$ NMR $\left(400 \mathrm{MHz}, \mathrm{CDCl}_{3}\right) \delta$ 0.59-0.74 (m, 2H), 0.98-1.06 (m, 1H), 1.25-1.34 (m, 1H), $1.58(\mathrm{~s}, 3 \mathrm{H}), 1.60-1.76(\mathrm{~m}, 2 \mathrm{H}), 2.43-2.49(\mathrm{~m}, 1 \mathrm{H}), 3.01-3.08$ $(\mathrm{m}, 1 \mathrm{H}), 3.75(\mathrm{~s}, 3 \mathrm{H}), 4.62(\mathrm{~d}, J=3.8 \mathrm{~Hz}, 1 \mathrm{H}), 7.21-7.33(\mathrm{~m}, 4 \mathrm{H}) ;{ }^{13} \mathrm{C}$ NMR $(100$ $\left.\mathrm{MHz}, \mathrm{CDCl}_{3}\right) \delta 14.1,27.3,28.2,29.0,46.0,47.1,51.3,63.2,64.0,124.0,124.3,126.6$, 127.7, 137.9, 139.1, 174.2; HRMS (ESI) $m / z$ : $[\mathrm{M}+\mathrm{H}]^{+}$Calcd for $\mathrm{C}_{16} \mathrm{H}_{20} \mathrm{NO}_{2}$ 258.1489, found 258.1491 .

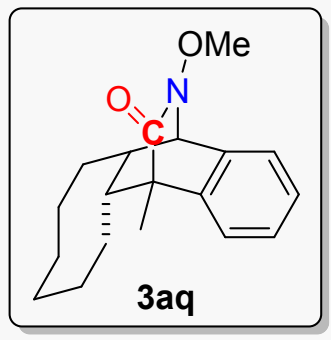

\section{4-Methoxy-12-methyl-5,5a,6,7,8,9,10,11,11a,12-decahydro-}

5,12-(epiminomethano)cycloocta[b]naphthalen-13-one (3aq): the title compound was prepared according to general procedure and purified by column chromatography (petroleum ether/ethyl acetate $=5 / 1$ to $3 / 1)$ to give a white solid $(102 \mathrm{mg}, 68 \%$ yield, m.p. $\left.130-133{ }^{\circ} \mathrm{C}\right) .{ }^{1} \mathbf{H}$ NMR $\left(400 \mathrm{MHz}, \mathrm{CDCl}_{3}\right) \delta$ 0.48-0.54 (m, 1H), 0.86-0.96 (m, $1 \mathrm{H}), 1.12-1.23(\mathrm{~m}, 2 \mathrm{H}), 1.28-1.50(\mathrm{~m}, 7 \mathrm{H}), 1.56-1.63(\mathrm{~m}, 1 \mathrm{H}), 1.66(\mathrm{~s}, 3 \mathrm{H}), 1.86(\mathrm{t}, J$ $=9.6 \mathrm{~Hz}, 1 \mathrm{H}), 2.49-2.56(\mathrm{~m}, 1 \mathrm{H}), 3.76(\mathrm{~s}, 3 \mathrm{H}), 4.44(\mathrm{~d}, J=2.8 \mathrm{~Hz}, 1 \mathrm{H}), 7.18-7.24(\mathrm{~m}$, 3H), 7.27-7.32 (m, 1H); ${ }^{13} \mathbf{C}$ NMR (100 MHz, $\left.\mathrm{CDCl}_{3}\right) \delta 14.2,23.3,26.0,26.2,29.1$, 30.9, 31.1, 44.4, 45.7, 52.9, 63.4, 67.9, 123.3, 123.3, 126.4, 127.6, 137.0, 139.2, 174.2; HRMS (ESI) $m / z$ : $[\mathrm{M}+\mathrm{H}]^{+}$Calcd for $\mathrm{C}_{19} \mathrm{H}_{26} \mathrm{NO}_{2} 300.1964$, found 300.1960.

2-(10-Methoxy-4-methyl-9-oxo-1,2,3,4-tetrahydro-1,4-(epiminomethano)naphtha len-3-yl)benzaldehyde $\boldsymbol{O}$-methyl oxime (4a): the title compound was prepared 


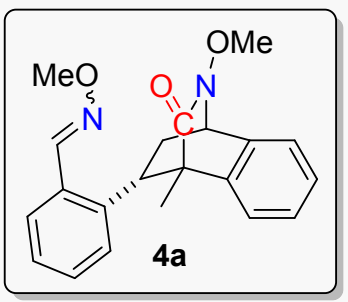

according to general procedure and purified by column chromatography (petroleum ether/ethyl acetate $=5 / 1$ to $3 / 1$ ) to give a white solid $\left(64.8 \mathrm{mg}, 74 \%\right.$ yield, m.p. $\left.144-146{ }^{\circ} \mathrm{C}\right)$. ${ }^{1} \mathbf{H}$ NMR $\left(400 \mathrm{MHz}, \mathrm{CDCl}_{3}\right) \delta 1.31(\mathrm{~s}, 3 \mathrm{H}), 1.72-1.77(\mathrm{~m}$, $1 \mathrm{H}), 2.94-3.01(\mathrm{~m}, 1 \mathrm{H}), 3.79-3.81(\mathrm{~m}, 1 \mathrm{H}), 3.83(\mathrm{~s}, 3 \mathrm{H}), 4.00$ (s, $3 \mathrm{H}), 4.95\left(\mathrm{dd}, J_{1}=3.6 \mathrm{~Hz}, J_{2}=1.6 \mathrm{~Hz}, 1 \mathrm{H}\right), 5.47\left(\mathrm{dd}, J_{1}=8.0 \mathrm{~Hz}, J_{2}=1.2 \mathrm{~Hz}, 1 \mathrm{H}\right)$, 6.89-6.93 (m, 1H), $6.98(\mathrm{~d}, J=7.2 \mathrm{~Hz}, 1 \mathrm{H}), 7.12\left(\mathrm{dt}, J_{l}=7.6 \mathrm{~Hz}, J_{2}=1.2 \mathrm{~Hz}, 1 \mathrm{H}\right)$, 7.28-7.40 (m, 3H), $7.61\left(\mathrm{dd}, J_{1}=8.0 \mathrm{~Hz}, J_{2}=1.6 \mathrm{~Hz}, 1 \mathrm{H}\right), 8.50(\mathrm{~s}, 1 \mathrm{H}) ;{ }^{13} \mathbf{C}$ NMR $\left(100 \mathrm{MHz}, \mathrm{CDCl}_{3}\right) \delta 13.5,38.7,38.8,53.1,60.6,62.1,63.4,121.5,125.1,126.9$, 127.0, 127.3, 127.5, 128.0, 129.3, 131.7, 137.6, 139.5, 140.1, 147.4, 173.1; HRMS (ESI) $m / z$ : $[\mathrm{M}+\mathrm{H}]^{+}$Calcd for $\mathrm{C}_{21} \mathrm{H}_{23} \mathrm{~N}_{2} \mathrm{O}_{3} 351.1709$, found 351.1702 .

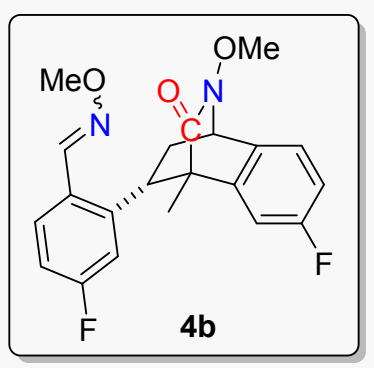

4-Fluoro-2-(6-fluoro-10-methoxy-4-methyl-9-oxo-1,2,3,4-t etrahydro-1,4-(epiminomethano)naphthalen-3-yl)benzald ehyde $O$-methyl oxime (4b): the title compound was prepared according to general procedure and purified by column chromatography (petroleum ether/ethyl acetate $=5 / 1$ to $3 / 1)$ to give a white solid (61.5 mg, $62 \%$ yield, m.p. $\left.123-129^{\circ} \mathrm{C}\right) .{ }^{1} \mathbf{H}$ NMR (400 $\left.\mathrm{MHz}, \mathrm{CDCl}_{3}\right) \delta 1.23(\mathrm{~s}, 3 \mathrm{H}), 1.61-1.66(\mathrm{~m}, 1 \mathrm{H}), 2.88-2.95(\mathrm{~m}, 1 \mathrm{H}), 3.75-3.79(\mathrm{~m}, 4 \mathrm{H})$, $3.92(\mathrm{~s}, 3 \mathrm{H}), 4.88\left(\mathrm{dd}, J_{1}=4.0 \mathrm{~Hz}, J_{2}=1.6 \mathrm{~Hz}, 1 \mathrm{H}\right), 5.10\left(\mathrm{dd}, J_{1}=10.7 \mathrm{~Hz}, J_{2}=2.7\right.$ $\mathrm{Hz}, 1 \mathrm{H}), 6.67\left(\mathrm{dd}, J_{1}=8.8 \mathrm{~Hz}, J_{2}=2.4 \mathrm{~Hz}, 1 \mathrm{H}\right), 6.77-6.82(\mathrm{~m}, 1 \mathrm{H}), 6.99-7.03(\mathrm{~m}, 1 \mathrm{H})$, $7.28\left(\mathrm{dd}, J_{1}=8.4 \mathrm{~Hz}, J_{2}=5.2 \mathrm{~Hz}, 1 \mathrm{H}\right), 7.54\left(\mathrm{dd}, J_{1}=8.8 \mathrm{~Hz}, J_{2}=6.0 \mathrm{~Hz}, 1 \mathrm{H}\right), 8.34(\mathrm{~s}$, $1 \mathrm{H}) ;{ }^{13} \mathrm{C}$ NMR $\left(100 \mathrm{MHz}, \mathrm{CDCl}_{3}\right) \delta 13.4,38.6,38.7,53.2,59.9,62.2,63.5,113.0(\mathrm{~d}$, $J=20 \mathrm{~Hz}), 113.6(\mathrm{~d}, J=20 \mathrm{~Hz}), 114.2(\mathrm{~d}, J=20 \mathrm{~Hz}), 114.5(\mathrm{~d}, J=20 \mathrm{~Hz}), 123.1(\mathrm{~d}$, $J=10 \mathrm{~Hz}), 128.0,129.5(\mathrm{~d}, J=10 \mathrm{~Hz}), 135.7,139.9(\mathrm{~d}, J=10 \mathrm{~Hz}), 141.8(\mathrm{~d}, J=10$ Hz), 146.3, 161.6 (d, $J=246 \mathrm{~Hz}), 161.8$ (d, $J=248 \mathrm{~Hz}), 172.3 ;{ }^{19}$ F NMR $(376 \mathrm{MHz}$, $\left.\mathrm{CDCl}_{3}\right) \delta-109.8,-112.5$; HRMS (ESI) $m / z$ : $[\mathrm{M}+\mathrm{Na}]^{+}$Calcd for $\mathrm{C}_{21} \mathrm{H}_{20} \mathrm{~N}_{2} \mathrm{NaO}_{3} \mathrm{~F}_{2}$ 409.1334, found 409.1334 .

\section{4-Chloro-2-(6-chloro-10-methoxy-4-methyl-9-oxo-1,2,3,4-tetrahydro-1,4-(epimin} omethano)naphthalen-3-yl)benzaldehyde $O$-methyl oxime $(4 c):$ the title compound was prepared according to general procedure and purified by column 


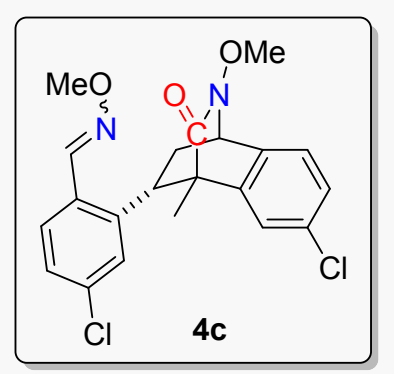

chromatography (petroleum ether/ethyl acetate $=5 / 1$ to $3 / 1$ ) to give a white solid $\left(60.7 \mathrm{mg}, 58 \%\right.$ yield, m.p. $\left.155-161^{\circ} \mathrm{C}\right)$. ${ }^{1} \mathbf{H}$ NMR $\left(400 \mathrm{MHz}, \mathrm{CDCl}_{3}\right) \delta 1.19(\mathrm{~s}, 3 \mathrm{H}), 1.58-1.64(\mathrm{~m}$, $1 \mathrm{H}), 2.86-2.93(\mathrm{~m}, 1 \mathrm{H}), 3.64\left(\mathrm{dd}, J_{1}=10.4 \mathrm{~Hz}, J_{2}=5.2 \mathrm{~Hz}\right.$, $1 \mathrm{H}), 3.74(\mathrm{~s}, 3 \mathrm{H}), 3.93(\mathrm{~s}, 3 \mathrm{H}), 4.83\left(\mathrm{dd}, J_{1}=4.0 \mathrm{~Hz}, J_{2}=1.6\right.$ $\mathrm{Hz}, 1 \mathrm{H}), 5.37$ (d, $J=8.0 \mathrm{~Hz}, 1 \mathrm{H}), 6.83-6.87(\mathrm{~m}, 2 \mathrm{H})$, 7.20-7.23 (m, 1H), $7.33(\mathrm{~d}, J=2.4 \mathrm{~Hz}, 1 \mathrm{H}), 7.58(\mathrm{~d}, J=2.4 \mathrm{~Hz}, 1 \mathrm{H}), 8.34(\mathrm{~s}, 1 \mathrm{H}) ;{ }^{13} \mathrm{C}$ NMR $\left(100 \mathrm{MHz}, \mathrm{CDCl}_{3}\right) \delta 13.5,38.2,38.3,52.7,60.1,62.3,63.6,122.2,126.5$, $127.0,128.1,128.2,129.4,133.1,133.4,133.5,135.9,137.4,141.5,145.7,172.4$; HRMS (ESI) $m / z$ : $[\mathrm{M}+\mathrm{Na}]^{+}$Calcd for $\mathrm{C}_{21} \mathrm{H}_{20} \mathrm{~N}_{2} \mathrm{NaO}_{3} \mathrm{Cl}_{2}$ 441.0743, found 441.0743 .

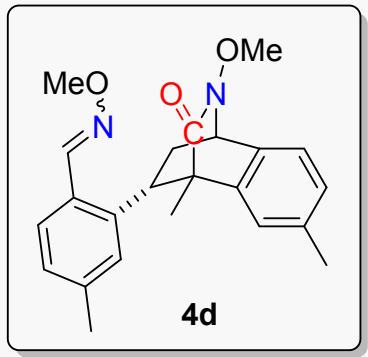

2-(10-Methoxy-4,6-dimethyl-9-oxo-1,2,3,4-tetrahydro-1,4(epiminomethano)naphthalen-3-yl)-4-methylbenzaldehyd e $\boldsymbol{O}$-methyl oxime (4d): the title compound was prepared according to general procedure and purified by column chromatography (petroleum ether/ethyl acetate $=5 / 1$ to $3 / 1$ ) to give a white solid $\left(72.0 \mathrm{mg}, 76 \%\right.$ yield, m.p. $\left.168-173{ }^{\circ} \mathrm{C}\right)$. ${ }^{1} \mathbf{H}$ NMR $\left(400 \mathrm{MHz}, \mathrm{CDCl}_{3}\right) \delta 1.26(\mathrm{~s}, 3 \mathrm{H}), 1.67-1.72(\mathrm{~m}, 1 \mathrm{H}), 1.19(\mathrm{~s}, 3 \mathrm{H}), 2.33(\mathrm{~s}$, $3 \mathrm{H}), 2.89-2.96(\mathrm{~m}, 1 \mathrm{H}), 3.75\left(\mathrm{dd}, J_{1}=10.0 \mathrm{~Hz}, J_{2}=4.8 \mathrm{~Hz}, 1 \mathrm{H}\right), 3.82(\mathrm{~s}, 3 \mathrm{H}), 3.98(\mathrm{~s}$, $3 \mathrm{H}), 4.91\left(\mathrm{dd}, J_{1}=3.6 \mathrm{~Hz}, J_{2}=2.0 \mathrm{~Hz}, 1 \mathrm{H}\right), 5.11-5.12(\mathrm{~m}, 1 \mathrm{H}), 6.77-6.78(\mathrm{~m}, 1 \mathrm{H})$, 6.91-6.94 (m, 1H), 7.16-7.19 (m, 1H), $7.26(\mathrm{~d}, J=7.2 \mathrm{~Hz}, 1 \mathrm{H}), 7.50(\mathrm{~d}, J=8.0 \mathrm{~Hz}$, 1H), $8.45(\mathrm{~s}, 1 \mathrm{H}) ;{ }^{13} \mathbf{C}$ NMR $\left(100 \mathrm{MHz}, \mathrm{CDCl}_{3}\right) \delta 13.5,21.3,21.5,38.8,39.0,52.3$, $60.5,62.1,63.4,121.2,126.1,127.1,127.7,127.8,128.1,129.0,13.4,137.7,137.9$, 139.2, 139.5, 147.5, 173.3. HRMS (ESI) $m / z$ : $[\mathrm{M}+\mathrm{Na}]^{+}$Calcd for $\mathrm{C}_{23} \mathrm{H}_{26} \mathrm{~N}_{2} \mathrm{NaO}_{3}$ 401.1836, found 401.1835 . 


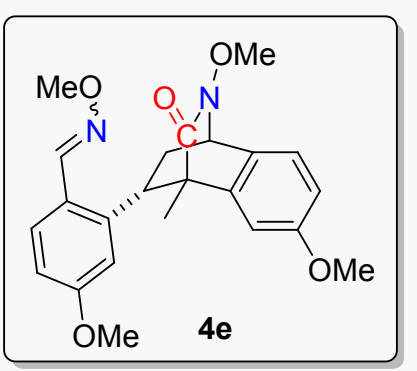

2-(6,10-Dimethoxy-4-methyl-9-oxo-1,2,3,4-tetrahydro1,4-(epiminomethano)naphthalen-3-yl)-4-methoxybenz aldehyde $O$-methyl oxime (4e) : the title compound was prepared according to general procedure and purified by column chromatography (petroleum ether/ethyl acetate $=5 / 1$ to $3 / 1)$ to give a white solid $(83.3 \mathrm{mg}, 81 \%$ yield, m.p. 168-171 $\left.{ }^{\circ} \mathrm{C}\right) .{ }^{1} \mathbf{H}$ NMR (400 MHz, $\left.\mathrm{CDCl}_{3}\right) \delta 1.18(\mathrm{~s}, 3 \mathrm{H}), 1.59-1.64(\mathrm{~m}, 1 \mathrm{H})$, 2.82-2.88 (m, 1H), $3.55\left(\mathrm{dd}, J_{1}=10.0 \mathrm{~Hz}, J_{2}=5.2 \mathrm{~Hz}, 1 \mathrm{H}\right), 3.69(\mathrm{~s}, 3 \mathrm{H}), 3.75(\mathrm{~s}, 3 \mathrm{H})$, $3.80(\mathrm{~s}, 3 \mathrm{H}), 3.92(\mathrm{~s}, 3 \mathrm{H}), 4.79\left(\mathrm{dd}, J_{l}=4.0 \mathrm{~Hz}, J_{2}=2.0 \mathrm{~Hz}, 1 \mathrm{H}\right), 5.35(\mathrm{~d}, J=8.8 \mathrm{~Hz}$, $1 \mathrm{H}), 6.44\left(\mathrm{dd}, J_{1}=8.8 \mathrm{~Hz}, J_{2}=2.8 \mathrm{~Hz}, 1 \mathrm{H}\right), 6.71\left(\mathrm{dd}, J_{1}=8.4 \mathrm{~Hz}, J_{2}=2.4 \mathrm{~Hz}, 1 \mathrm{H}\right)$, $6.79(\mathrm{~d}, J=8.4 \mathrm{~Hz}, 1 \mathrm{H}), 6.88(\mathrm{~d}, J=2.8 \mathrm{~Hz}, 1 \mathrm{H}), 7.10(\mathrm{~d}, J=2.8 \mathrm{~Hz}, 1 \mathrm{H}), 8.41(\mathrm{~s}$, $1 \mathrm{H}) ;{ }^{13} \mathrm{C}$ NMR $\left(100 \mathrm{MHz}, \mathrm{CDCl}_{3}\right) \delta 13.6,38.4,38.6,52.5,55.3,55.5,60.8,62.1$, $63.4,108.3,110.4,112.3,116.5,126.1,128.3,129.5,131.8,132.6,141.3,147.1$, 158.0, 159.2, 173.6; HRMS (ESI) $m / z$ : $[\mathrm{M}+\mathrm{Na}]^{+}$Calcd for $\mathrm{C}_{23} \mathrm{H}_{26} \mathrm{~N}_{2} \mathrm{NaO}_{5}$ 433.1734, found 433.1724 .

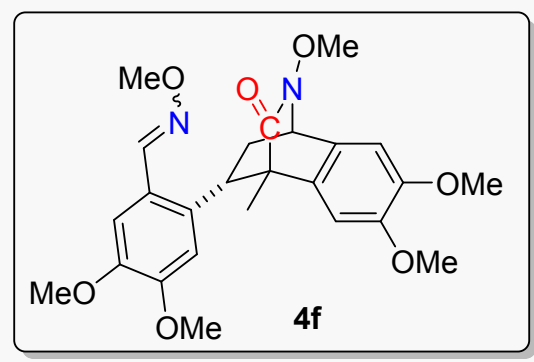

4,5-Dimethoxy-2-(6,7,10-trimethoxy-4-methyl-9oxo-1,2,3,4-tetrahydro-1,4-(epiminomethano)na phthalen-3-yl)benzaldehyde $O$-methyl oxime (4f): the title compound was prepared according to general procedure and purified by column chromatography (petroleum ether/ethyl acetate $=5 / 1$ to $3 / 1$ ) to give a white solid (88.3 mg, 75\% yield, m.p. 171-174 ${ }^{\circ} \mathrm{C}$ ). ${ }^{1} \mathbf{H}$ NMR (400 MHz, $\left.\mathrm{CDCl}_{3}\right) \delta 1.21$ (s, 3H), $1.62-1.66(\mathrm{~m}, 1 \mathrm{H}), 2.84-2.90(\mathrm{~m}, 1 \mathrm{H}), 3.19(\mathrm{~s}, 3 \mathrm{H}), 3.55\left(\mathrm{dd}, J_{l}=10.0 \mathrm{~Hz}, J_{2}=4.8 \mathrm{~Hz}\right.$, $1 \mathrm{H}), 3.72(\mathrm{~s}, 3 \mathrm{H}), 3.77(\mathrm{~s}, 3 \mathrm{H}), 3.80(\mathrm{~s}, 3 \mathrm{H}), 3.86(\mathrm{~s}, 3 \mathrm{H}), 3.91(\mathrm{~s}, 3 \mathrm{H}), 4.80\left(\mathrm{dd}, J_{l}=\right.$ $\left.3.6 \mathrm{~Hz}, J_{2}=1.6 \mathrm{~Hz}, 1 \mathrm{H}\right), 4.93(\mathrm{~s}, 1 \mathrm{H}), 6.50(\mathrm{~s}, 1 \mathrm{H}), 6.92(\mathrm{~s}, 1 \mathrm{H}), 7.13(\mathrm{~s}, 1 \mathrm{H}), 8.40(\mathrm{~s}$, $1 \mathrm{H}) ;{ }^{13} \mathrm{C}$ NMR $\left(100 \mathrm{MHz}, \mathrm{CDCl}_{3}\right) \delta 13.8,38.6,38.8,52.8,55.0,55.8,56.3,56.4$, 60.4, 62.0, 63.5, 106.0, 108.2, 109.2, 109.3, 124.1, 130.1, 132.6, 132.7, 146.5, 147.7, 148.4, 148.9, 149.8, 173.7; HRMS (ESI) $m / z$ : $[\mathrm{M}+\mathrm{Na}]^{+}$Calcd for $\mathrm{C}_{25} \mathrm{H}_{30} \mathrm{~N}_{2} \mathrm{NaO}_{7}$ 493.1945, found 493.1939. 


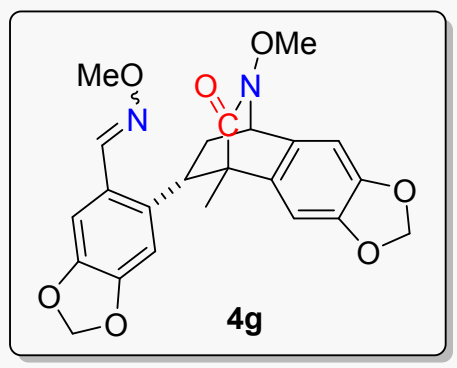

6-(11-Methoxy-8-methyl-10-oxo-5,6,7,8-tetrahydro-5,8 -(epiminomethano)naphtho[2,3-d][1,3]dioxol-7-yl)be nzo[d][1,3]dioxole-5-carbaldehyde $O$-methyl oxime (4g): the title compound was prepared according to general procedure and purified by column chromatography (petroleum ether/ethyl acetate $=5 / 1$ to $3 / 1$ ) to give a white solid (76.6 mg, 70\% yield, m.p. 209-215 ${ }^{\circ} \mathrm{C}$ ). ${ }^{1} \mathbf{H}$ NMR (400 MHz, $\left.\mathrm{CDCl}_{3}\right) \delta 1.19(\mathrm{~s}, 3 \mathrm{H})$, $1.50-1.57(\mathrm{~m}, 1 \mathrm{H}), 2.78-2.85(\mathrm{~m}, 1 \mathrm{H}), 3.50\left(\mathrm{dd}, J_{1}=10.0 \mathrm{~Hz}, J_{2}=5.2 \mathrm{~Hz}, 1 \mathrm{H}\right), 3.74(\mathrm{~s}$ 3H), 3.89 (s, 3H), 4.73 (d, $J=3.6 \mathrm{~Hz}, 1 \mathrm{H}), 5.04$ (s, 1H), 5.79 (d, $J=14.4 \mathrm{~Hz}, 2 \mathrm{H})$, $5.91(\mathrm{~d}, J=16.0 \mathrm{~Hz}, 2 \mathrm{H}), 6.49(\mathrm{~s}, 1 \mathrm{H}), 6.82(\mathrm{~s}, 1 \mathrm{H}), 7.12(\mathrm{~s}, 1 \mathrm{H}), 8.34(\mathrm{~s}, 1 \mathrm{H}) ;{ }^{13} \mathrm{C}$ NMR $\left(100 \mathrm{MHz}, \mathrm{CDCl}_{3}\right) \delta 13.9,38.6,38.6,52.8,60.4,62.0,63.4,101.3,101.3$, $103.5,106.0,106.6,125.5,131.1,133.8,134.3,146.5,146.7,146.8,147.5,148.9$, 173.5; HRMS (ESI) $m / z$ : $[\mathrm{M}+\mathrm{Na}]^{+}$Calcd for $\mathrm{C}_{23} \mathrm{H}_{22} \mathrm{~N}_{2} \mathrm{NaO}_{7}$ 461.1319, found 461.1321.

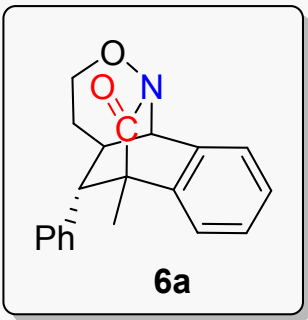

${ }^{1} \mathbf{H}$ NMR $\left(400 \mathrm{MHz}, \mathrm{CDCl}_{3}\right) \delta 1.30(\mathrm{~s}, 3 \mathrm{H}), 1.69-1.74(\mathrm{~m}, 1 \mathrm{H}), 2.36-2.45(\mathrm{~m}, 2 \mathrm{H})$, $2.65(\mathrm{~d}, J=3.2 \mathrm{~Hz}, 1 \mathrm{H}), 3.79-3.84(\mathrm{~m}, 1 \mathrm{H}), 4.35-4.43(\mathrm{~m}, 1 \mathrm{H}), 4.68(\mathrm{~d}, J=1.6 \mathrm{~Hz}$, $1 \mathrm{H}), 6.14-6.17(\mathrm{~m}, 2 \mathrm{H}), 6.83\left(\mathrm{dd}, J_{1}=7.6 \mathrm{~Hz}, J_{2}=1.2 \mathrm{~Hz}, 1 \mathrm{H}\right), 6.95-6.99(\mathrm{~m}, 2 \mathrm{H})$, 7.04-7.08 (m, 1H), $7.23\left(\mathrm{dt}, J_{1}=7.6 \mathrm{~Hz}, J_{2}=1.6 \mathrm{~Hz}, 1 \mathrm{H}\right), 7.30\left(\mathrm{dt}, J_{1}=7.6 \mathrm{~Hz}, J_{2}=\right.$ $1.2 \mathrm{~Hz}, 1 \mathrm{H}), 7.35\left(\mathrm{dd}, J_{l}=7.2 \mathrm{~Hz}, J_{2}=1.6 \mathrm{~Hz}, 1 \mathrm{H}\right) ;{ }^{13} \mathbf{C ~ N M R}\left(100 \mathrm{MHz}, \mathrm{CDCl}_{3}\right) \delta$ $13.8,29.3,42.6,53.6,54.1,63.2,65.4,123.7,124.7,127.2,127.5,127.9,128.7,136.2$, 136.9, 139.3, 180.4; HRMS (ESI) $m / z$ : $[\mathrm{M}+\mathrm{Na}]^{+}$Calcd for $\mathrm{C}_{20} \mathrm{H}_{19} \mathrm{NNaO}_{2} 328.1308$, found 328.1310 .
7-Methyl-12-phenyl-2,3,7,11b-tetrahydro-1,7-methano[1,2]0 xazino[3,2-a]isoquinolin-6(1H)-one $(6 \mathbf{6})$ : the title compound was prepared according to general procedure and purified by column chromatography (petroleum ether/ethyl acetate $=5 / 1$ to 3/1) to give a white solid (107 mg, 70\% yield, m.p. $268-271{ }^{\circ} \mathrm{C}$ ). 


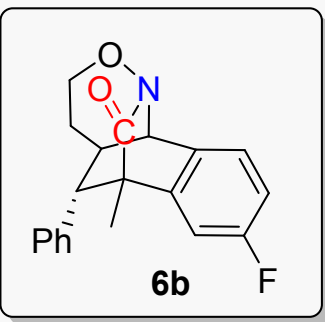

9-Fluoro-7-methyl-12-phenyl-2,3,7,11b-tetrahydro-1,7-met hano[1,2]oxazino[3,2-a]isoquinolin-6(1H)-one $(6 \mathrm{~b})$ : the title compound was prepared according to general procedure and purified by column chromatography (petroleum ether/ethyl acetate $=5 / 1$ to $3 / 1)$ to give a white solid $(90.7 \mathrm{mg}, 56 \%$ yield, m.p. $\left.247-250{ }^{\circ} \mathrm{C}\right) .{ }^{1} \mathbf{H}$ NMR $\left(400 \mathrm{MHz}, \mathrm{CDCl}_{3}\right) \delta 1.38(\mathrm{~s}, 3 \mathrm{H}), 1.79-1.84(\mathrm{~m}, 1 \mathrm{H})$, 2.45-2.57 (m, 2H), $2.65(\mathrm{~d}, J=3.2 \mathrm{~Hz}, 1 \mathrm{H}), 3.90\left(\mathrm{dd}, J_{l}=12.4 \mathrm{~Hz}, J_{2}=6.0 \mathrm{~Hz}, 1 \mathrm{H}\right)$, $4.47\left(\mathrm{dt}, J_{1}=12.4 \mathrm{~Hz}, J_{2}=6.0 \mathrm{~Hz}, 1 \mathrm{H}\right), 4.96(\mathrm{~d}, J=1.6 \mathrm{~Hz}, 1 \mathrm{H}), 6.29-6.31(\mathrm{~m}, 2 \mathrm{H})$, $6.66\left(\mathrm{dd}, J_{1}=8.8 \mathrm{~Hz}, J_{2}=2.4 \mathrm{~Hz}, 1 \mathrm{H}\right), 7.06-7.21(\mathrm{~m}, 4 \mathrm{H}), 7.43\left(\mathrm{dd}, J_{1}=8.0 \mathrm{~Hz}, J_{2}=\right.$ $5.2 \mathrm{~Hz}, 1 \mathrm{H}) ;{ }^{13} \mathbf{C}$ NMR $\left(100 \mathrm{MHz}, \mathrm{CDCl}_{3}\right) \delta$ 13.8, 29.3, 42.6, 53.4, 54.3, 63.2, 64.7, $112.4(\mathrm{~d}, J=23 \mathrm{~Hz}), 114.1(\mathrm{~d}, J=22 \mathrm{~Hz}), 125.4(\mathrm{~d}, J=8 \mathrm{~Hz}), 127.5,128.1,128.6$, $132.8(\mathrm{~d}, J=3 \mathrm{~Hz}), 138.8,138.9,162.0(\mathrm{~d}, J=246 \mathrm{~Hz}), 179.6 ;{ }^{19}$ F NMR $(376 \mathrm{MHz}$, $\left.\mathrm{CDCl}_{3}\right) \delta$-111.8; HRMS (ESI) $m / z$ : $[\mathrm{M}+\mathrm{Na}]^{+}$Calcd for $\mathrm{C}_{20} \mathrm{H}_{18} \mathrm{FNNaO}_{2}$ 346.1214, found 346.1208 .

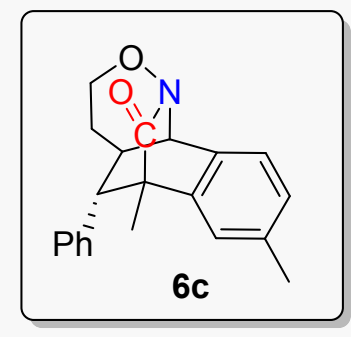

\section{9-Methyl-7-methyl-12-phenyl-2,3,7,11b-tetrahydro-1,7-met} hano[1,2] oxazino[3,2-a]isoquinolin-6(1H)-one $(6 \mathrm{c})$ : the title compound was prepared according to general procedure and purified by column chromatography (petroleum ether/ethyl acetate $=5 / 1$ to $3 / 1)$ to give a white solid $(100 \mathrm{mg}, 63 \%$ yield, m.p. $\left.228-231{ }^{\circ} \mathrm{C}\right) .{ }^{1} \mathbf{H}$ NMR $\left(400 \mathrm{MHz}, \mathrm{CDCl}_{3}\right) \delta 1.38(\mathrm{~s}, 3 \mathrm{H}), 1.77-1.83(\mathrm{~m}, 1 \mathrm{H})$, $2.33(\mathrm{~s}, 3 \mathrm{H}), 2.44-2.53(\mathrm{~m}, 2 \mathrm{H}), 2.72(\mathrm{~d}, J=3.2 \mathrm{~Hz}, 1 \mathrm{H}), 3.89\left(\mathrm{dd}, J_{1}=12.4 \mathrm{~Hz}, J_{2}=\right.$ $6.0 \mathrm{~Hz}, 1 \mathrm{H}), 4.44-4.51(\mathrm{~m}, 1 \mathrm{H}), 4.91(\mathrm{~d}, J=1.6 \mathrm{~Hz}, 1 \mathrm{H}), 6.27-6.29(\mathrm{~m}, 2 \mathrm{H}), 6.75$ (d, $J$ $=1.2 \mathrm{~Hz}, 1 \mathrm{H}), 7.06-7.21(\mathrm{~m}, 4 \mathrm{H}), 7.34(\mathrm{~d}, J=7.6 \mathrm{~Hz}, 1 \mathrm{H}) ;{ }^{13} \mathbf{C}$ NMR $(100 \mathrm{MHz}$, $\left.\mathrm{CDCl}_{3}\right) \delta 13.8,21.6,29.4,42.8,53.7,54.0,63.1,65.1,123.5,125.4,127.2,127.9$, 128.0, 128.8, 134.1, 136.0, 138.5, 139.5, 180.5; HRMS (ESI) $m / z:[\mathrm{M}+\mathrm{Na}]^{+}$Calcd for $\mathrm{C}_{21} \mathrm{H}_{21} \mathrm{NNaO}_{2}$ 342.1464, found 342.1469. 


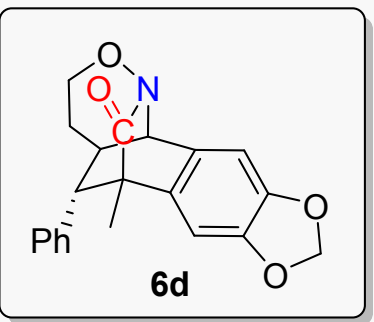

7-Methyl-13-phenyl-2,3,7,12b-tetrahydro-1,7-methano[1,3 ]dioxolo[4,5-g][1,2]oxazino[3,2-a]isoquinolin-6(1H)-one

(6d): the title compound was prepared according to general procedure and purified by column chromatography (petroleum ether/ethyl acetate $=5 / 1$ to $3 / 1$ ) to give a white

solid (59.3 mg, 34\% yield, m.p. 169-172 $\left.{ }^{\circ} \mathrm{C}\right) .{ }^{1} \mathbf{H}$ NMR $\left(400 \mathrm{MHz}, \mathrm{CDCl}_{3}\right) \delta 1.24$ (s, $3 \mathrm{H}), 1.68-1.73(\mathrm{~m}, 1 \mathrm{H}), 2.32-2.45(\mathrm{~m}, 2 \mathrm{H}), 2.60(\mathrm{~d}, J=2.8 \mathrm{~Hz}, 1 \mathrm{H}), 3.79\left(\mathrm{dd}, J_{l}=\right.$ $\left.12.4 \mathrm{~Hz}, J_{2}=5.6 \mathrm{~Hz}, 1 \mathrm{H}\right), 4.30-4.38(\mathrm{~m}, 1 \mathrm{H}), 4.74(\mathrm{~d}, J=1.6 \mathrm{~Hz}, 1 \mathrm{H}), 5.89(\mathrm{~d}, J=1.6$ $\mathrm{Hz}, 1 \mathrm{H}), 5.95$ (d, $J=1.6 \mathrm{~Hz}, 1 \mathrm{H}), 6.28-6.30(\mathrm{~m}, 2 \mathrm{H}), 6.35(\mathrm{~s}, 1 \mathrm{H}), 6.86(\mathrm{~s}, 1 \mathrm{H})$, 7.01-7.30 (m, 3H); ${ }^{13} \mathbf{C}$ NMR (100 MHz, $\left.\mathrm{CDCl}_{3}\right) \delta 14.3,29.4,42.8,53.5,53.7,63.0$, $65.3,101.3,105.2,106.2,127.3,128.1,128.7,130.1,130.5,139.4,146.8,148.1$, 180.5; HRMS (ESI) m/z: $[\mathrm{M}+\mathrm{Na}]^{+}$Calcd for $\mathrm{C}_{21} \mathrm{H}_{19} \mathrm{NNaO}_{4}$ 372.1206, found 372.1199 .

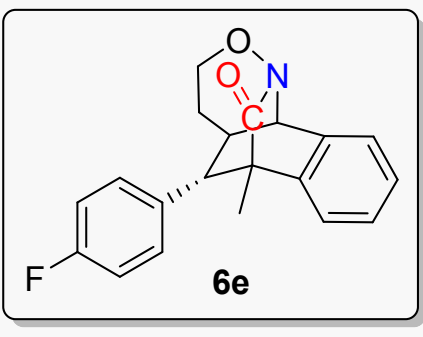

12-(4-Fluorophenyl)-7-methyl-2,3,7,11b-tetrahydro-1, 7-methano[1,2]oxazino[3,2-a]isoquinolin-6(1H)-one (6e): the title compound was prepared according to general procedure and purified by column chromatography (petroleum ether/ethyl acetate $=5 / 1$ to

3/1) to give a white solid (84.0 mg, 52\% yield, m.p. $\left.210-213{ }^{\circ} \mathrm{C}\right) .{ }^{\mathbf{1}} \mathbf{H}$ NMR $(400 \mathrm{MHz}$, $\left.\mathrm{CDCl}_{3}\right) \delta 1.38(\mathrm{~s}, 3 \mathrm{H}), 1.76-1.83(\mathrm{~m}, 1 \mathrm{H}), 2.45-2.55(\mathrm{~m}, 2 \mathrm{H}), 2.74(\mathrm{~d}, J=3.2 \mathrm{~Hz}, 1 \mathrm{H})$, $3.92\left(\mathrm{dd}, J_{1}=12.0 \mathrm{~Hz}, J_{2}=6.0 \mathrm{~Hz}, 1 \mathrm{H}\right), 4.43-4.51(\mathrm{~m}, 1 \mathrm{H}), 4.94(\mathrm{~d}, J=1.6 \mathrm{~Hz}, 1 \mathrm{H})$, 6.19 (br, 2H), 6.73-6.77 (m, 2H), $6.94\left(\mathrm{dd}, J_{1}=7.2 \mathrm{~Hz}, J_{2}=0.8 \mathrm{~Hz}, 1 \mathrm{H}\right), 7.28-7.46(\mathrm{~m}$, 3H); ${ }^{13} \mathrm{C}$ NMR $\left(100 \mathrm{MHz}, \mathrm{CDCl}_{3}\right) \delta 13.8,29.3,42.8,52.9,54.0,63.1,65.2,114.9(\mathrm{~d}$, $J=21 \mathrm{~Hz}), 123.7,124.7,127.6,128.8,130.0(\mathrm{~d}, J=8 \mathrm{~Hz}), 135.0(\mathrm{~d}, J=4 \mathrm{~Hz}), 135.9$, 136.8, $160.8(\mathrm{~d}, J=245 \mathrm{~Hz}), 180.2 ;{ }^{19} \mathbf{F}$ NMR $\left(376 \mathrm{MHz}, \mathrm{CDCl}_{3}\right) \delta-115.3$; HRMS (ESI) $m / z$ : $[\mathrm{M}+\mathrm{Na}]^{+}$Calcd for $\mathrm{C}_{20} \mathrm{H}_{18} \mathrm{FNNaO}_{2} 346.1214$, found 346.1211 . 


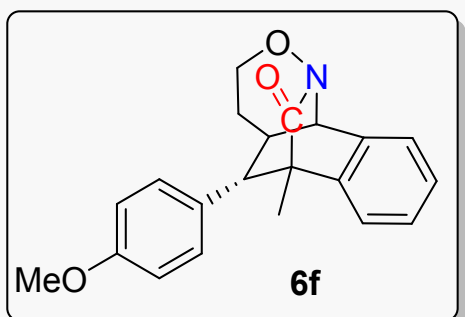

12-(4-Methoxyphenyl)-7-methyl-2,3,7,11b-tetrahyd ro-1,7-methano[1,2]oxazino[3,2-a]isoquinolin-6(1H) -one (6f): the title compound was prepared according to general procedure and purified by column chromatography (petroleum ether/ethyl acetate $=5 / 1$

to $3 / 1)$ to give a yellow solid $\left(98.8 \mathrm{mg}, 59 \%\right.$ yield, m.p. $\left.198-202{ }^{\circ} \mathrm{C}\right) .{ }^{1} \mathbf{H}$ NMR (400 $\left.\mathrm{MHz}, \mathrm{CDCl}_{3}\right) \delta 1.39(\mathrm{~s}, 3 \mathrm{H}), 1.77-1.82(\mathrm{~m}, 1 \mathrm{H}), 2.45-2.53(\mathrm{~m}, 2 \mathrm{H}), 2.71(\mathrm{~d}, J=2.8$ $\mathrm{Hz}, 1 \mathrm{H}), 3.71(\mathrm{~s}, 3 \mathrm{H}), 3.87-3.92(\mathrm{~m}, 1 \mathrm{H}), 4.44-4.51(\mathrm{~m}, 1 \mathrm{H}), 4.94(\mathrm{~d}, J=1.6 \mathrm{~Hz}, 1 \mathrm{H})$, 6.15-6.17 (m, 2H), $6.61\left(\mathrm{dd}, J_{1}=7.6 \mathrm{~Hz}, J_{2}=1.6 \mathrm{~Hz}, 2 \mathrm{H}\right), 6.95\left(\mathrm{dd}, J_{1}=7.2 \mathrm{~Hz}, J_{2}=\right.$ $1.2 \mathrm{~Hz}, 1 \mathrm{H}), 7.28-7.45(\mathrm{~m}, 3 \mathrm{H}) ;{ }^{13} \mathbf{C}$ NMR $\left(100 \mathrm{MHz}, \mathrm{CDCl}_{3}\right) \delta 13.8,29.3,42.7,52.9$, $54.2,55.1,63.2,65.4,113.3,123.6,124.7,127.4,128.6,129.6,131.3,136.3,136.9$, 158.7, 180.5; HRMS (ESI) $m / z$ : $[\mathrm{M}+\mathrm{Na}]^{+}$Calcd for $\mathrm{C}_{21} \mathrm{H}_{21} \mathrm{NNaO}_{3} 358.1414$, found 358.1402 . 


\section{X-ray crystal structure determination of the products}

Diffraction data for the complexes were collected with a Bruker SMART APEX II area detector at low temperature $(100 \mathrm{~K})$ with graphite-monochromated Mo $\mathrm{K} \alpha$ radiation $(\lambda=0.71073 \AA)$. An empirical absorption correction using SADABS $^{7}$ was applied for all data. The structure was solved by direct methods using the SHELXS program. All non-hydrogen atoms were refined anisotropically by full-matrix least-squares on $F^{2}$ by the use of the program SHELXL ${ }^{8}$. Crystallographic data for the complexes are summarized in Table S12. CCDC 2089533 (3aa) 2089534 (4a) and 2089532 (6a) contain the supplementary crystallographic data for the structures reported in this paper. Copies of the data can be obtained free of charge from The Cambridge Crystallographic Data Centre via www.ccdc.cam.ac.uk/data_request/cif.

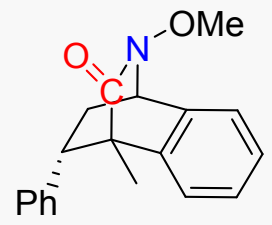

3aa

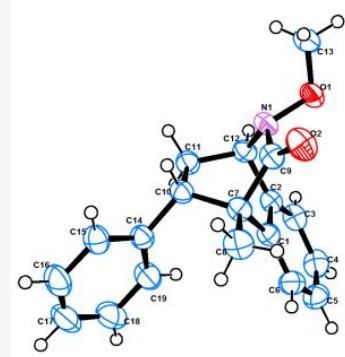

3aa

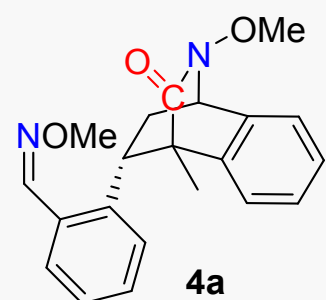

4a

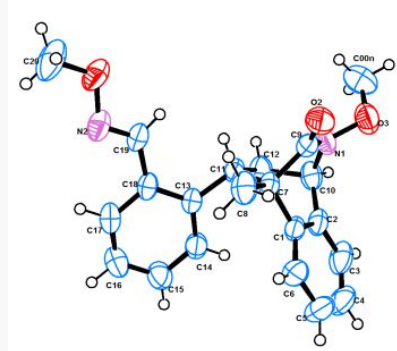

$4 \mathbf{a}$

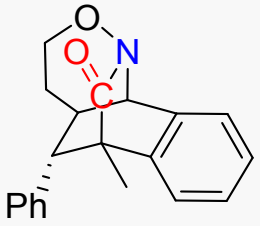

$6 a$

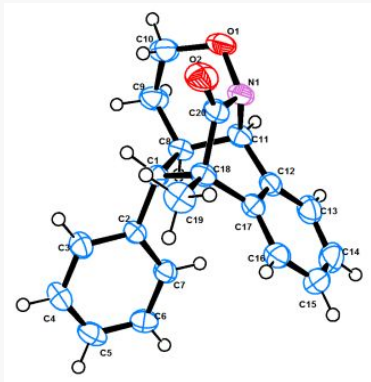

6a

Figure S3. The X-ray Diffraction Configuration of 3aa, 4a and 6a. Thermal ellipsoids are set at $50 \%$ probability. Crystal of compound 3aa, $4 \mathbf{a}$ and $6 \mathbf{a}$ was grown by slow evaporation of its solution with petroleum ether/ethyl acetate under $-20{ }^{\circ} \mathrm{C}$. 
Table S11. Crystallographic data for compounds 3aa, 4a and 6a.

\begin{tabular}{|c|c|c|c|}
\hline Compound & $3 \mathbf{a a}$ & $4 a$ & $6 \mathbf{a}$ \\
\hline formula & $\mathrm{C}_{19} \mathrm{H}_{19} \mathrm{NO}_{2}$ & $\mathrm{C}_{21} \mathrm{H}_{22} \mathrm{~N}_{2} \mathrm{O}_{3}$ & $\mathrm{C}_{20} \mathrm{H}_{19} \mathrm{NO}_{2}$ \\
\hline$F_{\mathrm{W}}$ & 293.14 & 350.40 & 305.36 \\
\hline crystal system & orthorhombic & orthorhombic & orthorhombic \\
\hline space group & $P 2(1) / c$ & $P 2(1) / c$ & $P 2(1) / c$ \\
\hline$a / \AA$ & $27.3660(4)$ & $13.2407(2)$ & $19.904(5)$ \\
\hline$b / \AA$ & $15.6473(2)$ & $7.61120(10)$ & $11.336(3)$ \\
\hline$c / \AA$ & $7.17560(10)$ & $18.5177(3)$ & $6.9814(17)$ \\
\hline$\alpha /{ }^{\circ}$ & 90 & 90 & 90 \\
\hline$\beta /{ }^{\circ}$ & 90 & $96.3760(10)$ & 90 \\
\hline$\gamma /{ }^{\circ}$ & 90 & 90 & 90 \\
\hline$V / \AA^{3}$ & $3072.62(7)$ & $1854.63(5)$ & $1575.3(7)$ \\
\hline$Z$ & 13 & 4 & 4 \\
\hline$D_{\text {calc }} / \mathrm{g} \mathrm{cm}^{-3}$ & 1.268 & 1.255 & 1.288 \\
\hline$F(000)$ & 1248 & 744 & 648 \\
\hline$\mu / \mathrm{mm}^{-1}$ & 0.651 & 0.682 & 0.083 \\
\hline$\theta$ range ${ }^{\circ}$ & $5.614 \sim 73.767$ & $3.907 \sim 73.686$ & $2.046 \sim 31.041$ \\
\hline $\begin{array}{l}\text { reflns collected } \\
\qquad\left(R_{\text {int }}\right)\end{array}$ & 7301 & 6830 & 10347 \\
\hline independent reflns & 3027 & 3634 & 4023 \\
\hline $\begin{array}{c}\text { observed reflns }[I> \\
2 \sigma(I)]\end{array}$ & 2687 & 3154 & 3415 \\
\hline$R_{1} ; w R_{2}[I>2 \sigma(I)]$ & $\begin{array}{l}0.0435 \\
0.1658\end{array}$ & $\begin{array}{l}0.0714, \\
0.1945\end{array}$ & $\begin{array}{l}0.0414, \\
0.1110\end{array}$ \\
\hline$R_{1} ; \mathrm{w} R_{2}$ (all data) & $\begin{array}{l}0.0479 \\
0.1786\end{array}$ & $\begin{array}{l}0.0768, \\
0.2041\end{array}$ & $\begin{array}{l}0.0513 \\
0.1193\end{array}$ \\
\hline $\operatorname{GOF}\left(F^{2}\right)$ & 0.802 & 1.059 & 0.997 \\
\hline
\end{tabular}




\section{References}

1. Shrestha, B.; Basnet, P.; Dhungana, R. K.; KC, S.; Thapa, S.; Sears, J. M.; Giri, R. J. Am. Chem. Soc. 2017, 139, 10653-10656.

2. Jarrige, L.; Carboni, A.; Dagousset, G.; Levitre, G.; Magnier, E.; Masson, G. Org. Lett. 2016, 18, 2906-2909.

3. Hibino, S.; Sugino, E.; Adachi, Y.; Nomi, K.; Sato, K. Heterocycles 1989, 28, 275-282.

4. Mou, X.-Q.; Rong, F.-M.; Zhang, H.; Chen, G.; He, G. Org. Lett. 2019, 21, 4657-4661.

5. Chen, J.; Guo, H.-M.; Zhao, Q.-Q.; Chen, J.-R.; Xiao, W.-J. Chem. Commun. 2018, 54, 6780-6783.

6. Cornella, J.; Martin, R. Org. Lett. 2013, 15, 6298-6301.

7. Sheldrick, G. M. Program SADABS: Area-Detector Absorption Correction, University of Gottingen, Germany, 1996.

8. Sheldrick, G. M. SHELXS-97, SHELXL-97, Programs for Crystal Structure Analysis, University of Gottingen, Germany, 1997. 
11. Copies of NMR for the staring materials and products
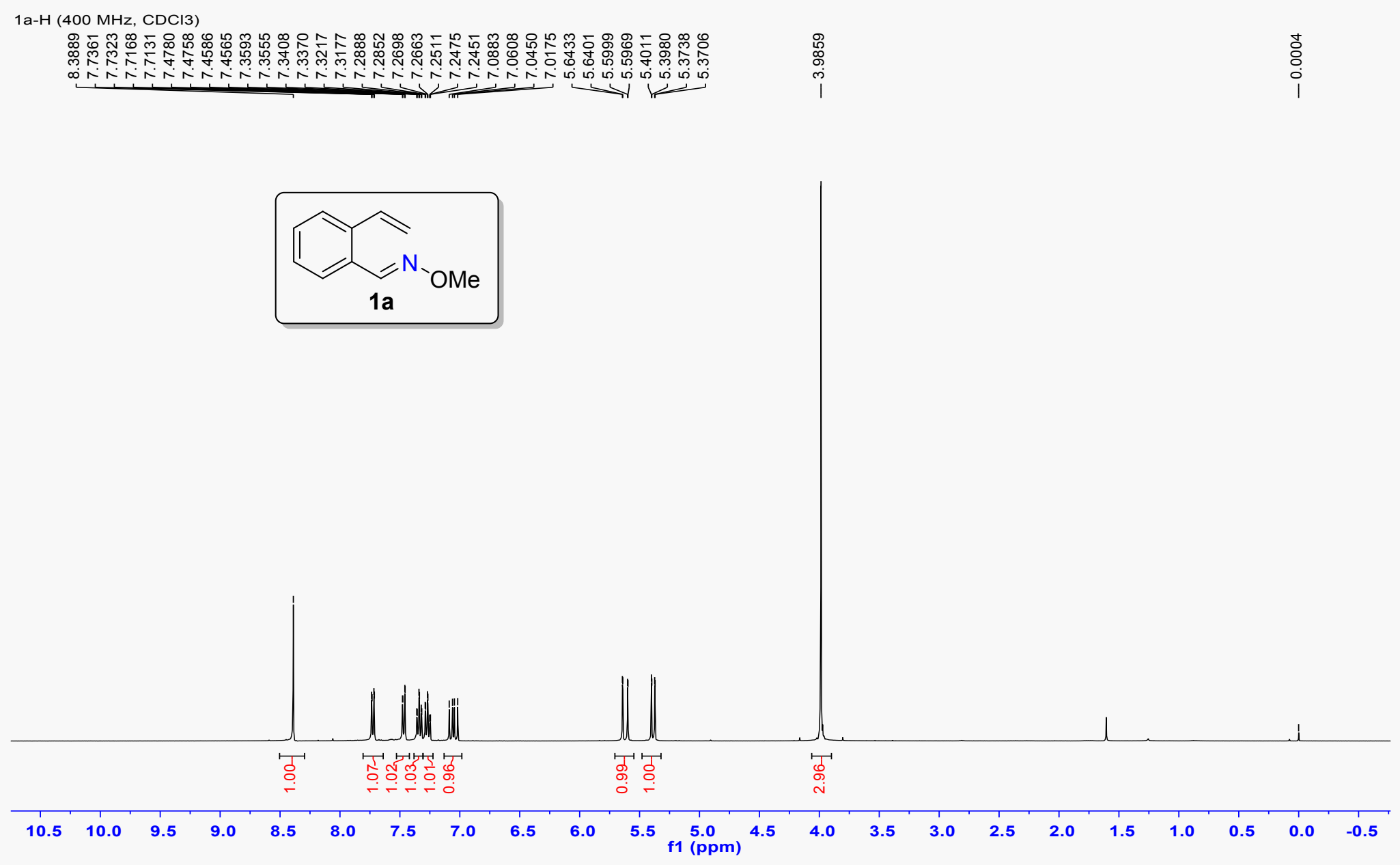
1a-C (100 MHz, CDCl3)

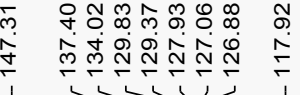

可供

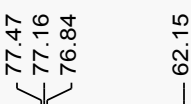
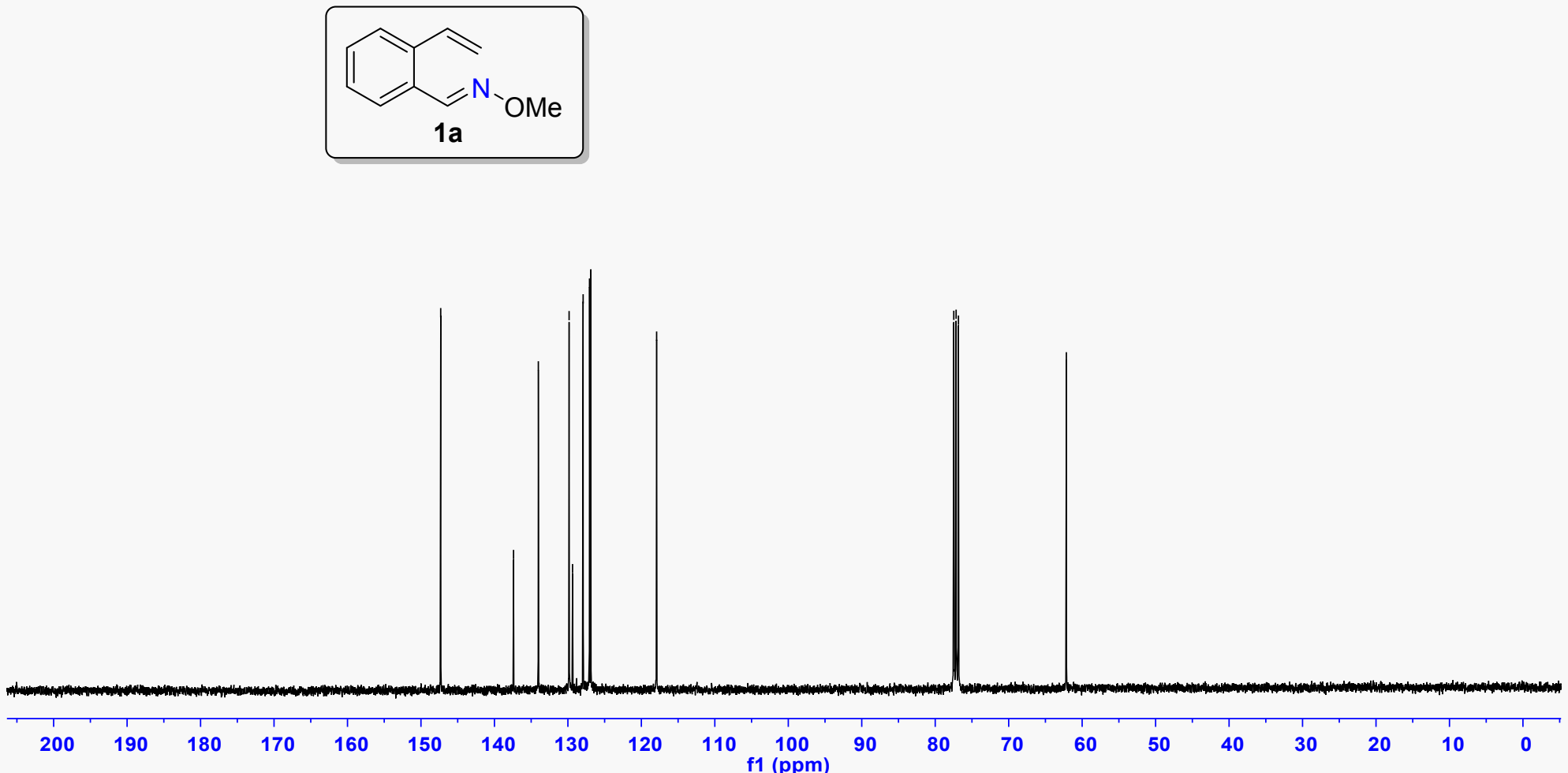
1b-H (400 MHz, CDCl3)

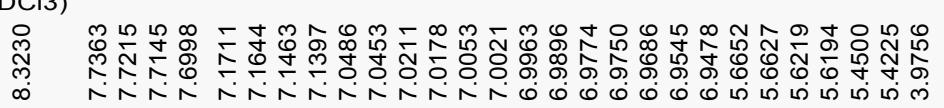

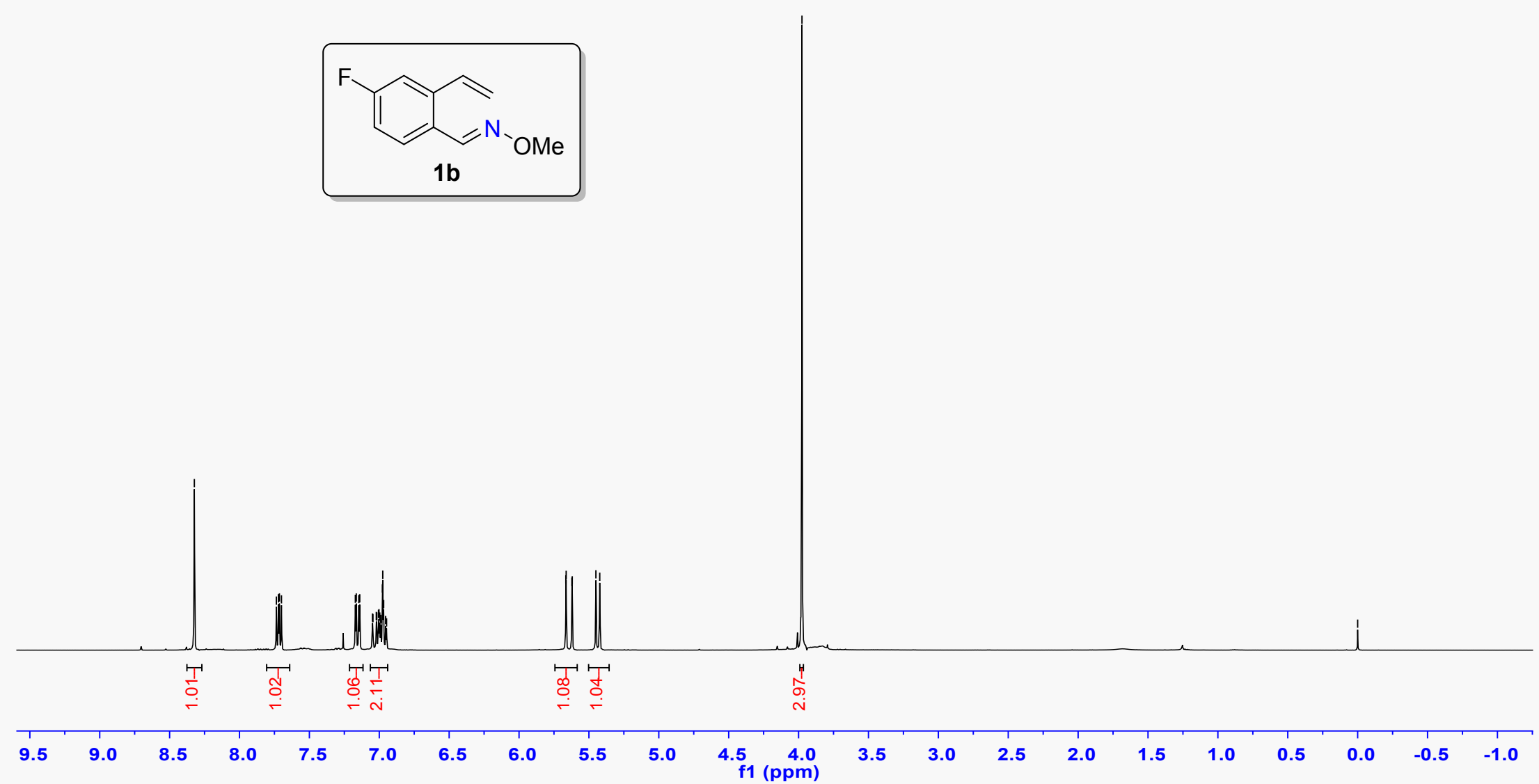


1b-C (100 MHz, CDCl3)

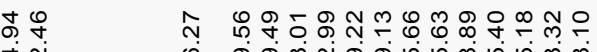

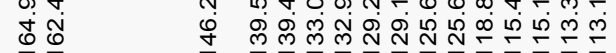

11 (1)

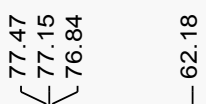
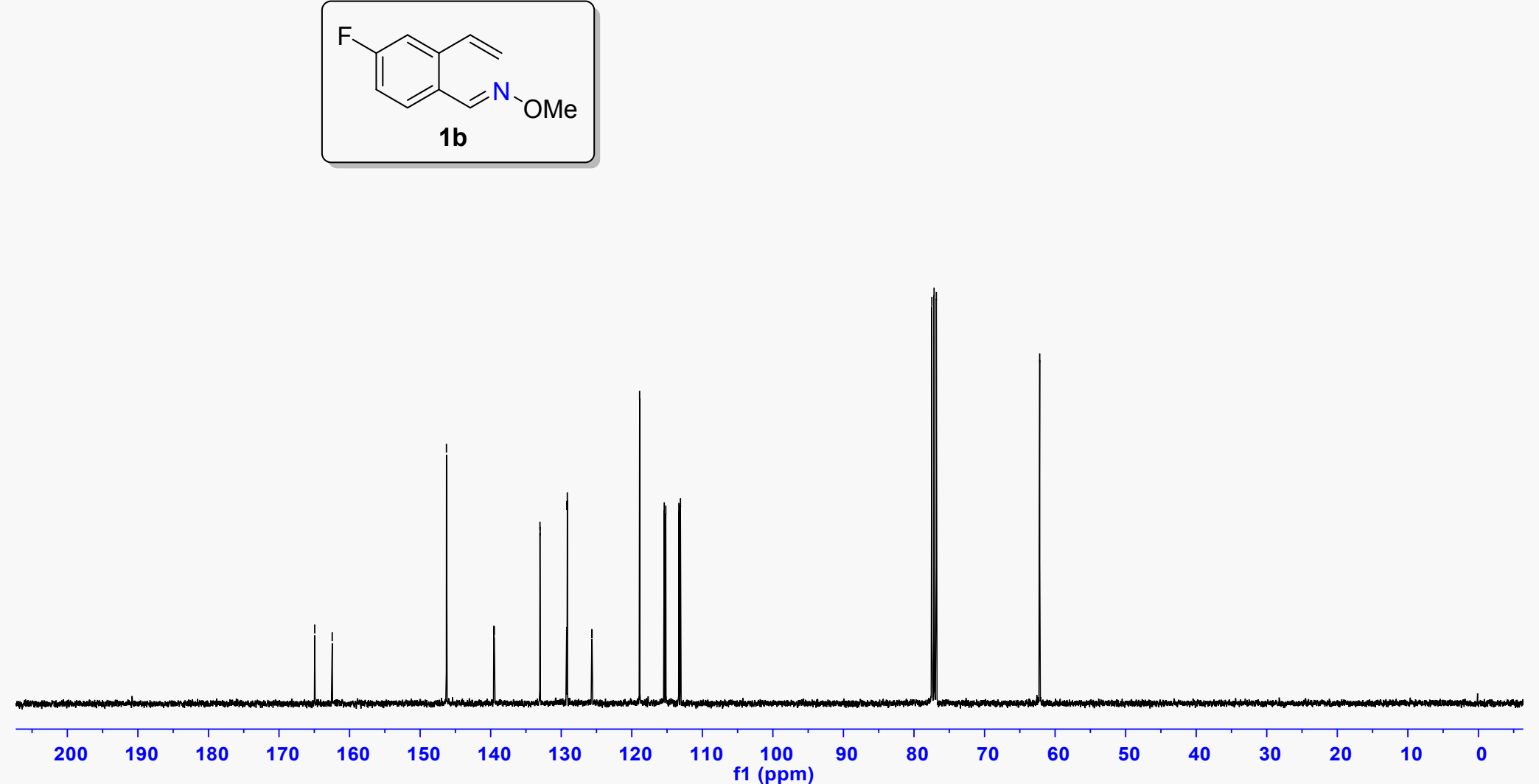
1d-F (376 MHz, CDCl3)
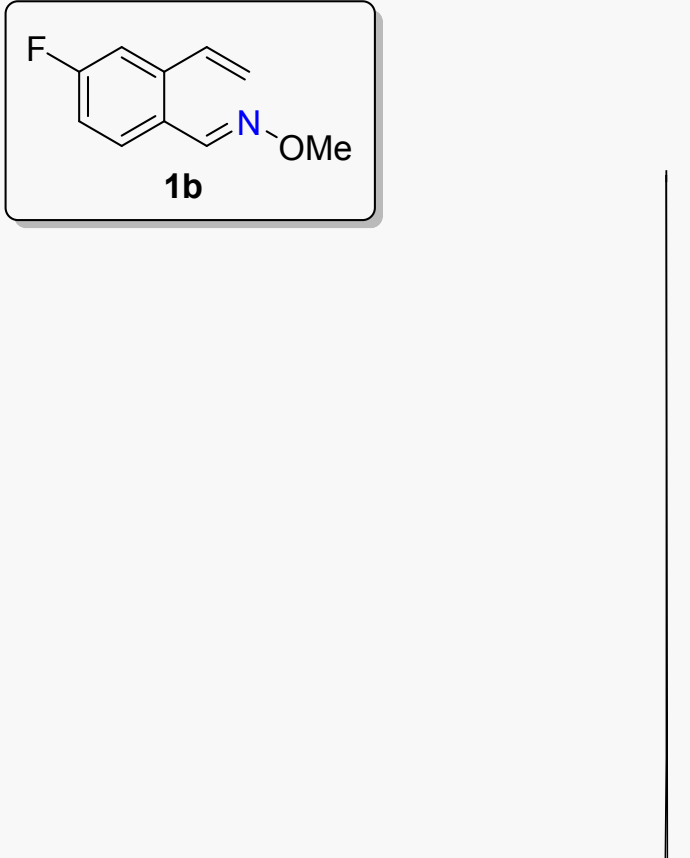
1C-H (400 MHz, CDCl3)
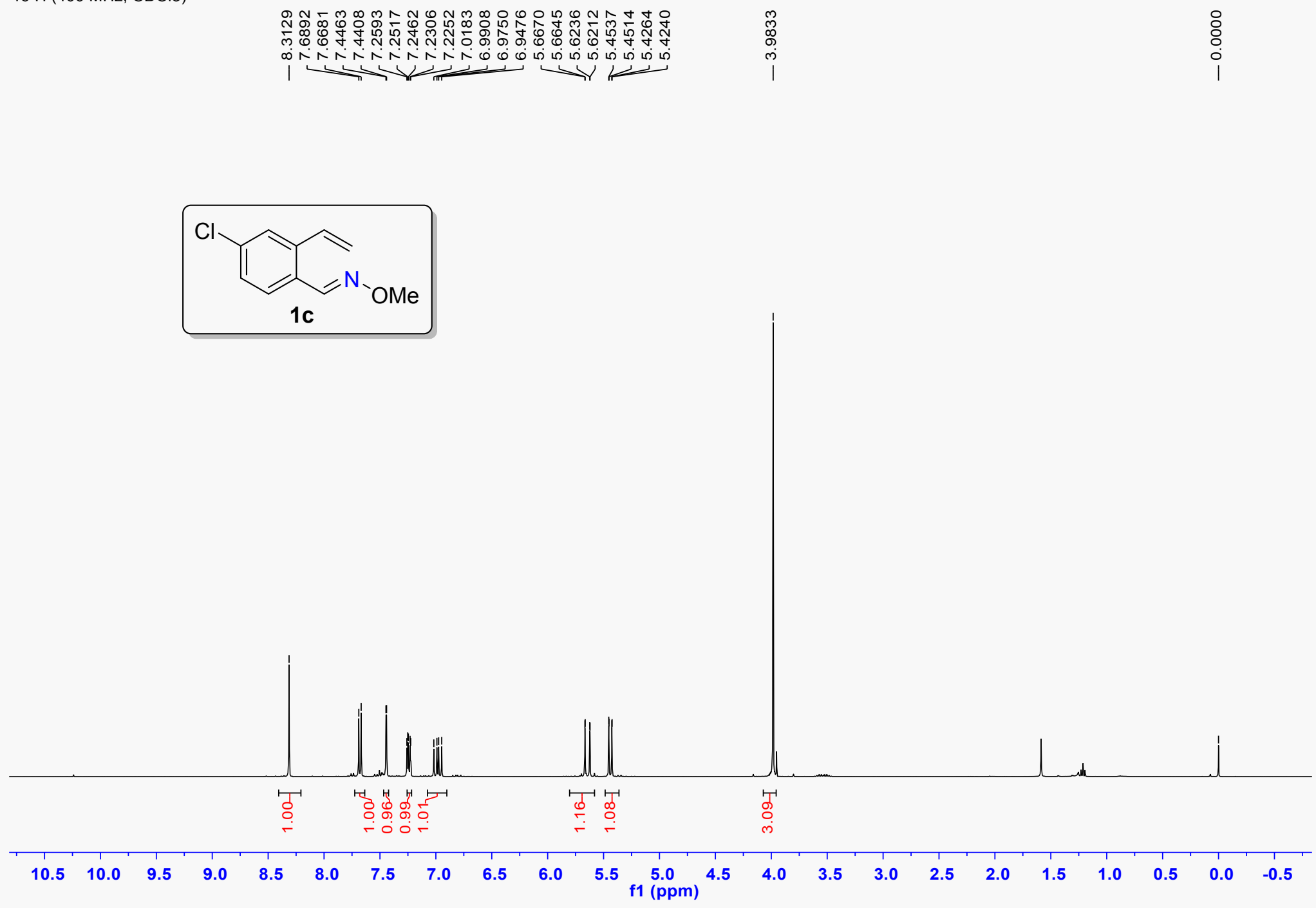
1c-C (100 MHz, CDCl3)

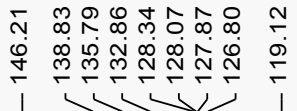

ริ

令是

กิ

। >
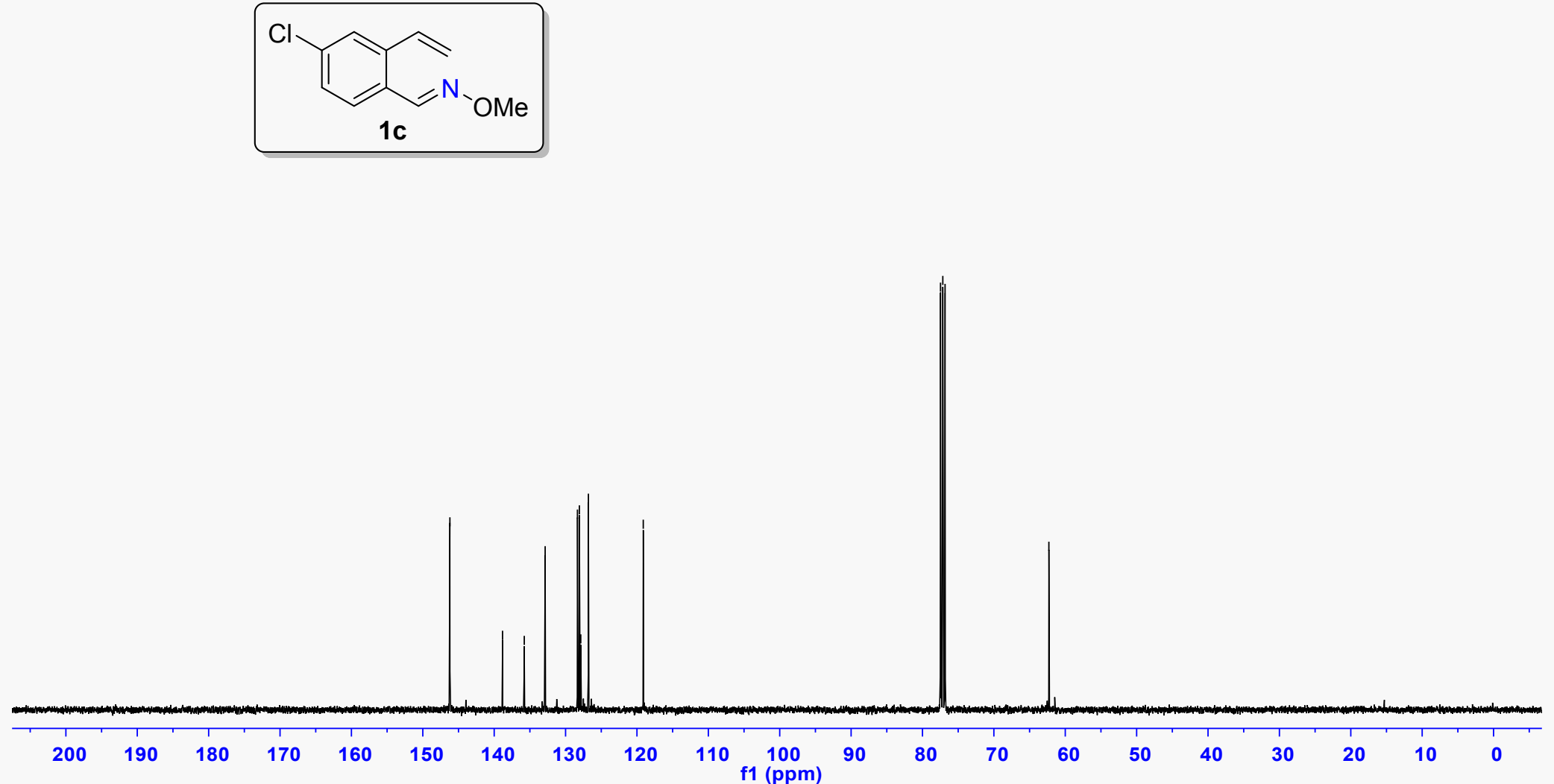
1d-H (400 MHz, CDCl3)
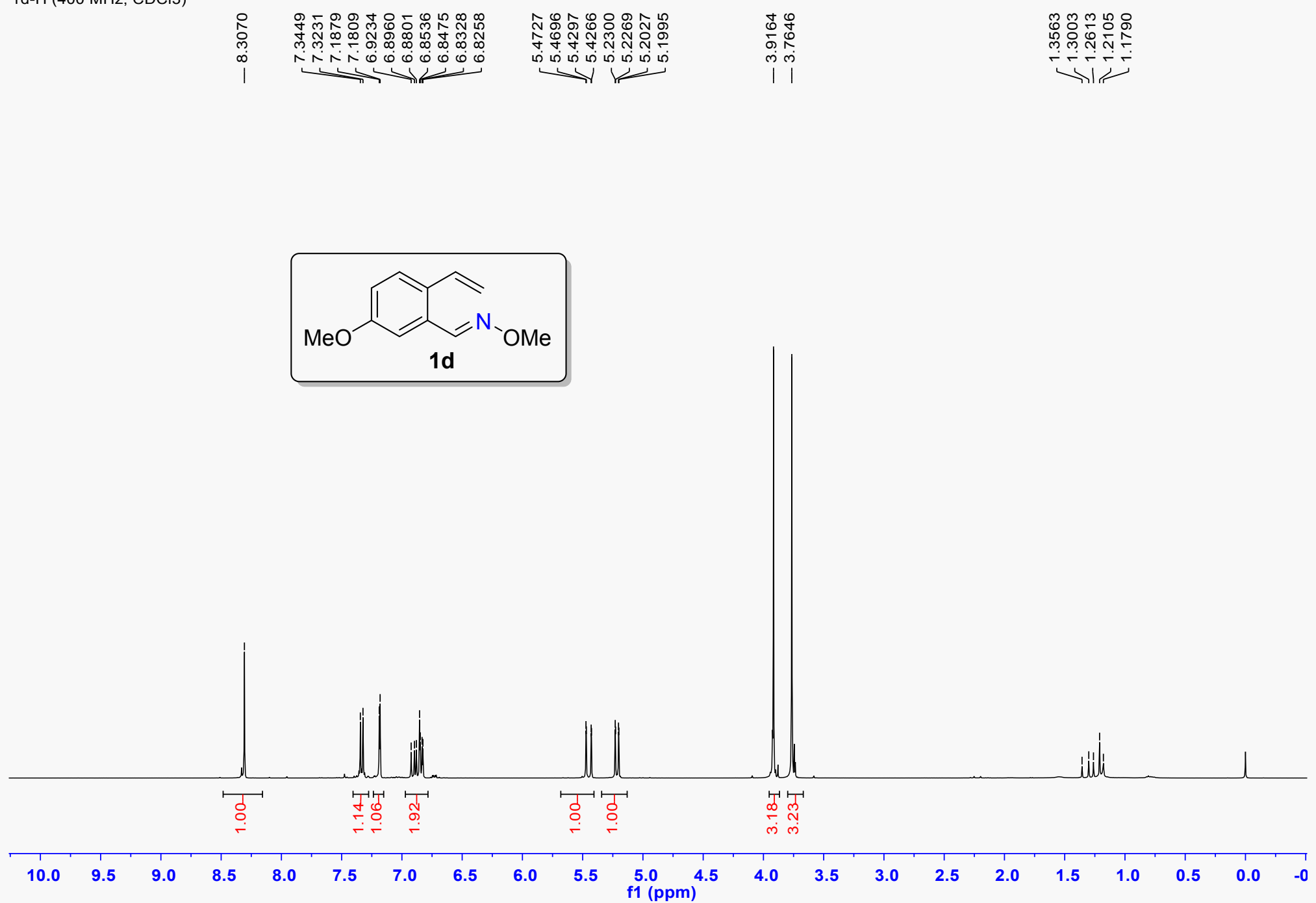
1d-C(100 MHz, CDCl3)

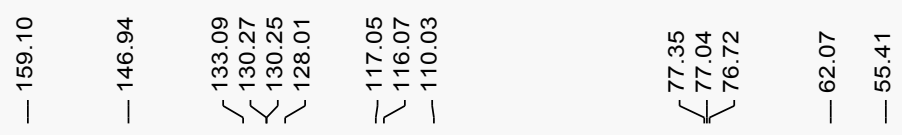
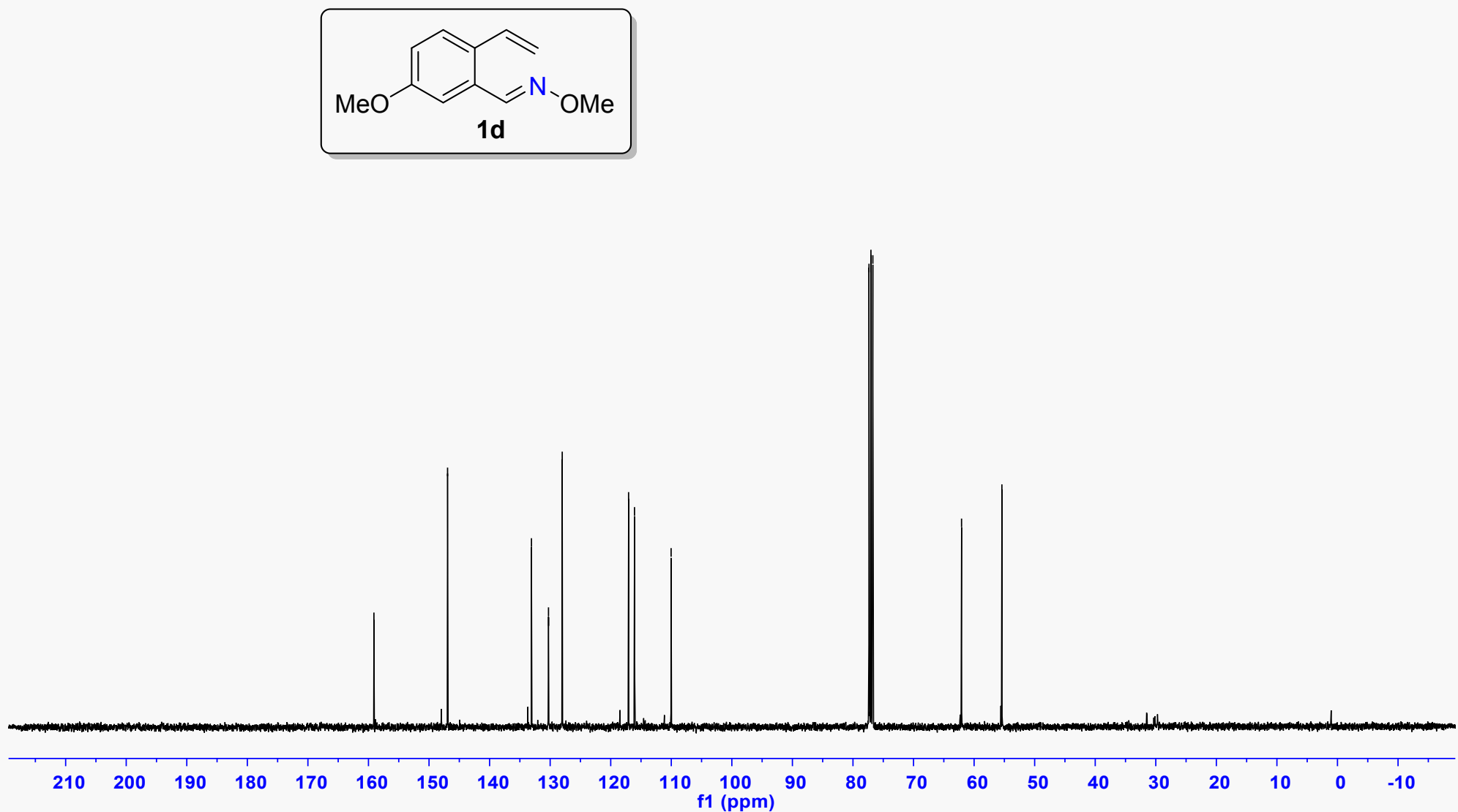
1e-H (400 MHz, CDCl3)
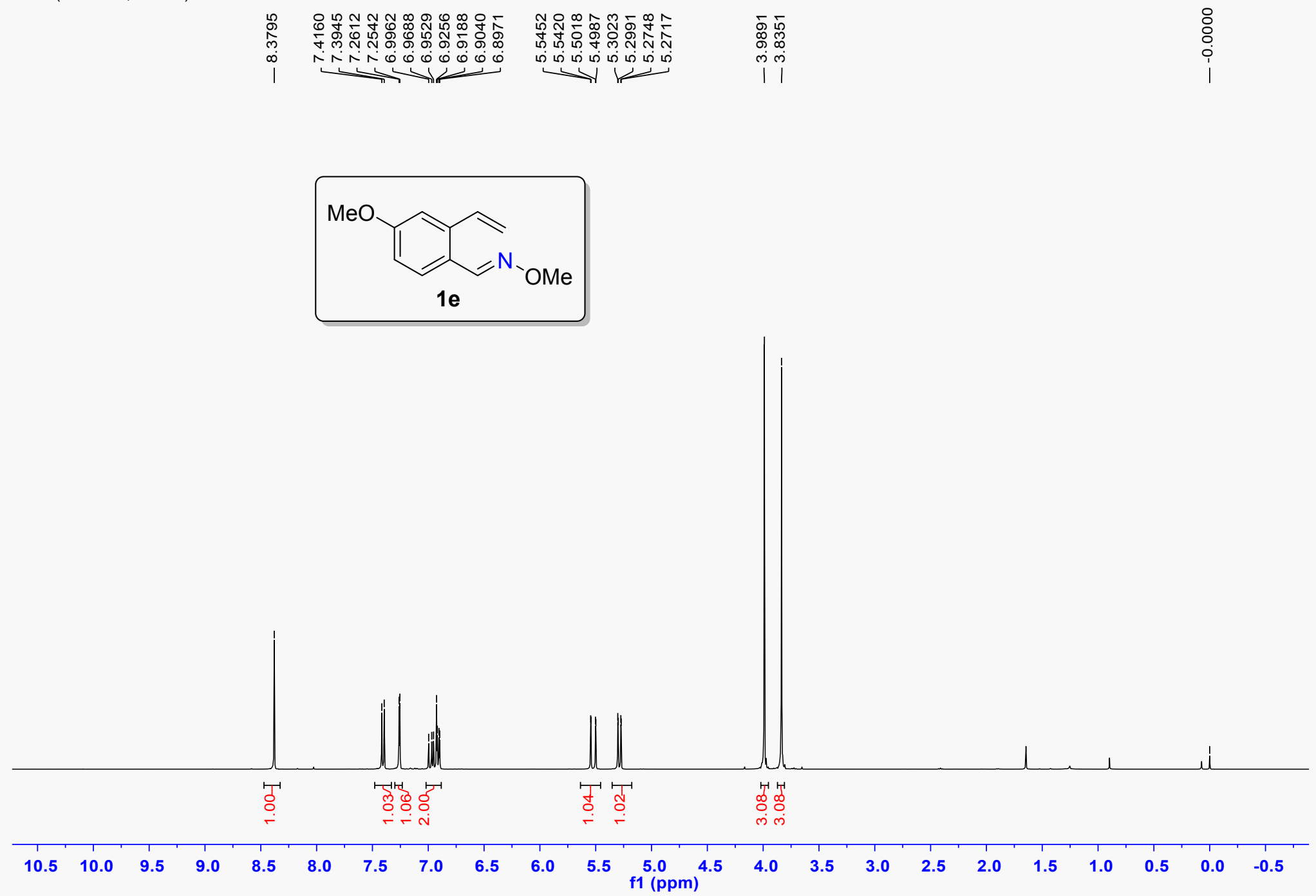
1e-C (100 MHz, CDCI3)

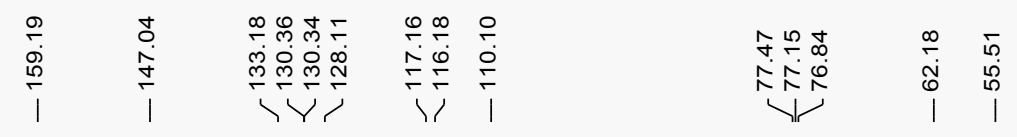
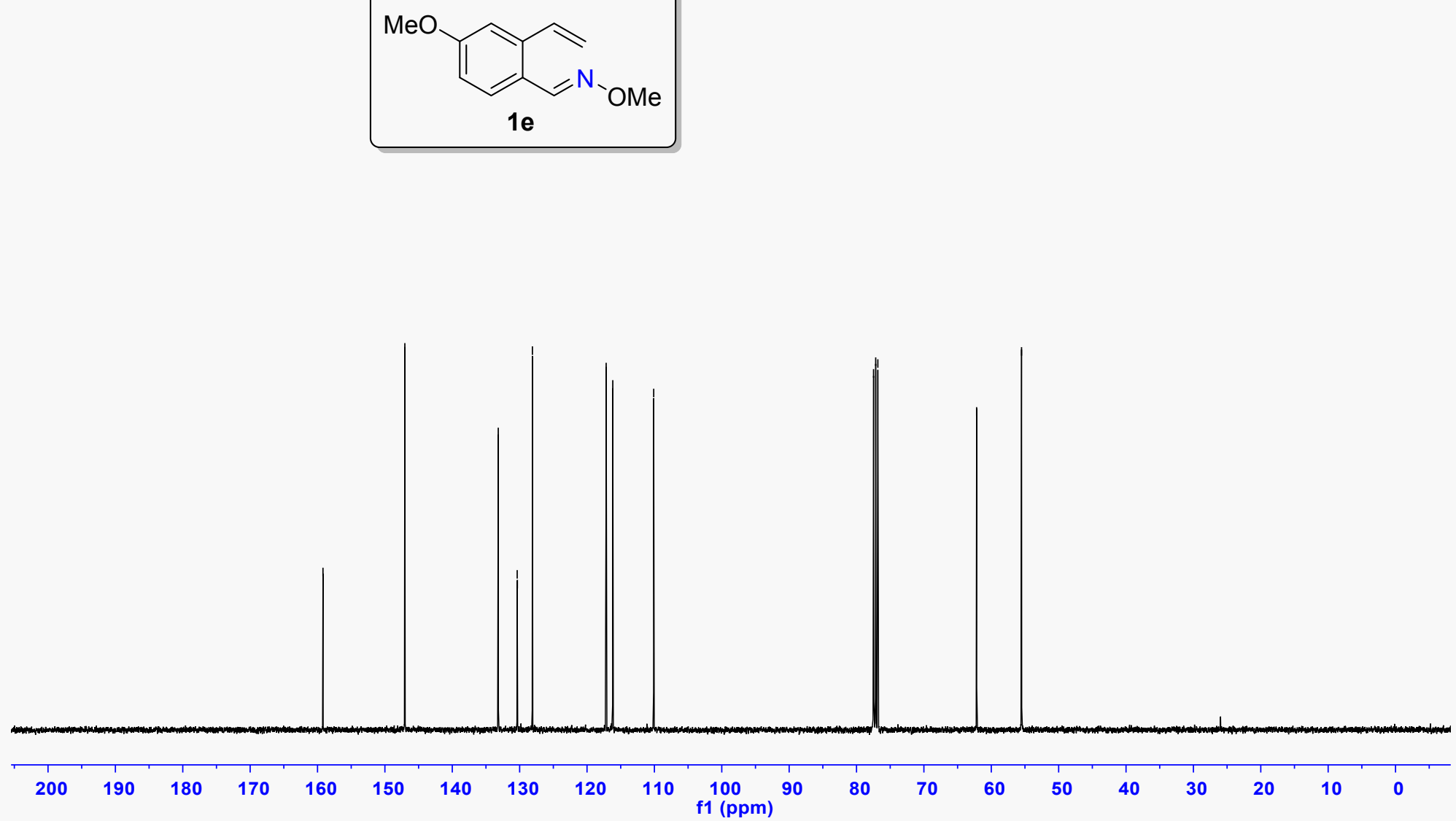
1f-H (400 MHz, CDCl3)
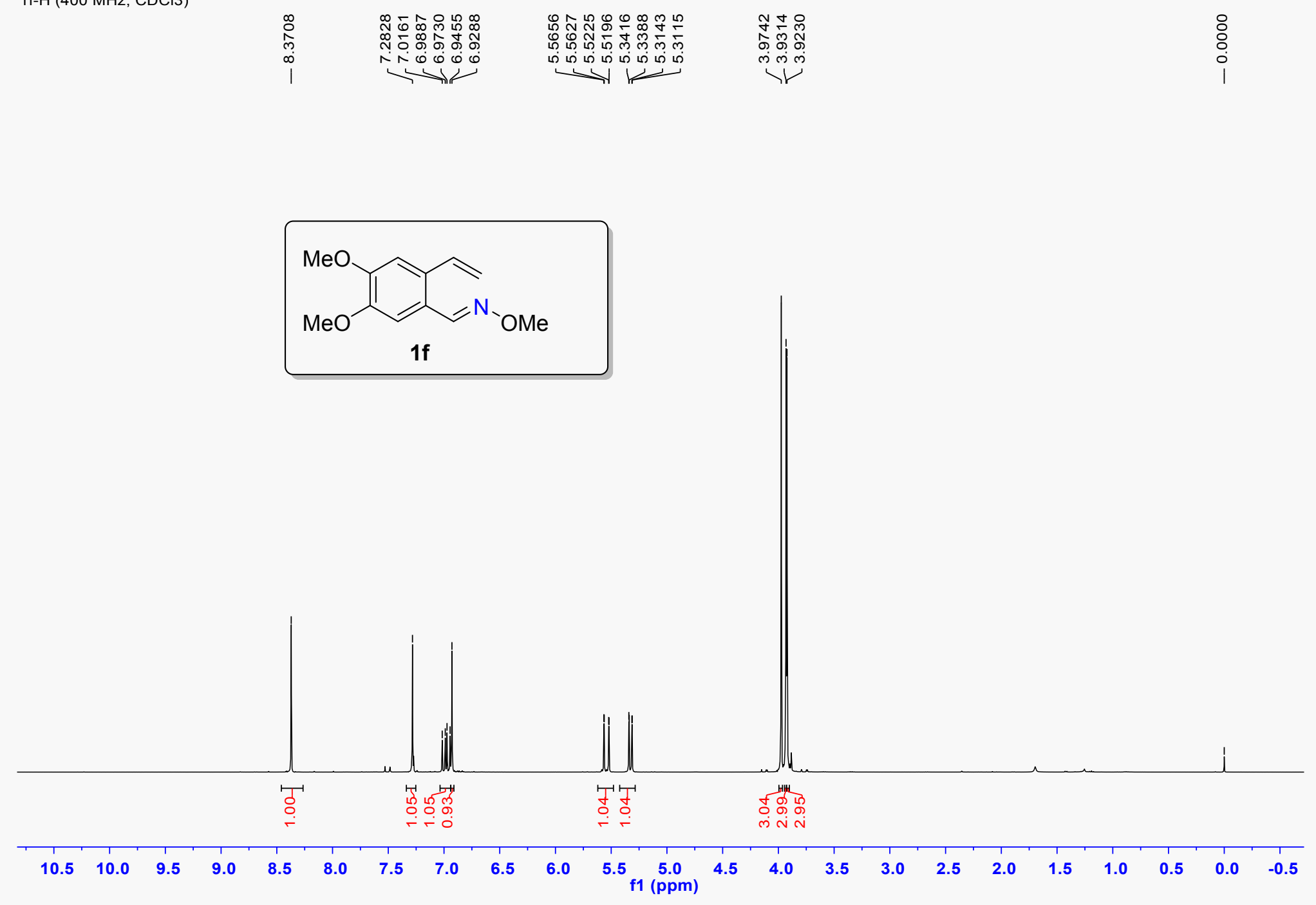
1f-C (100 MHz, CDCl3)

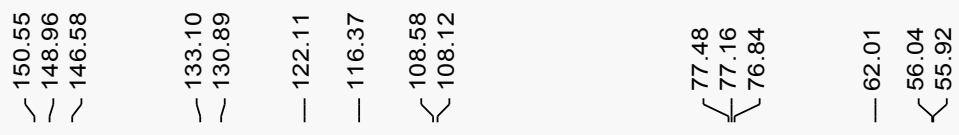
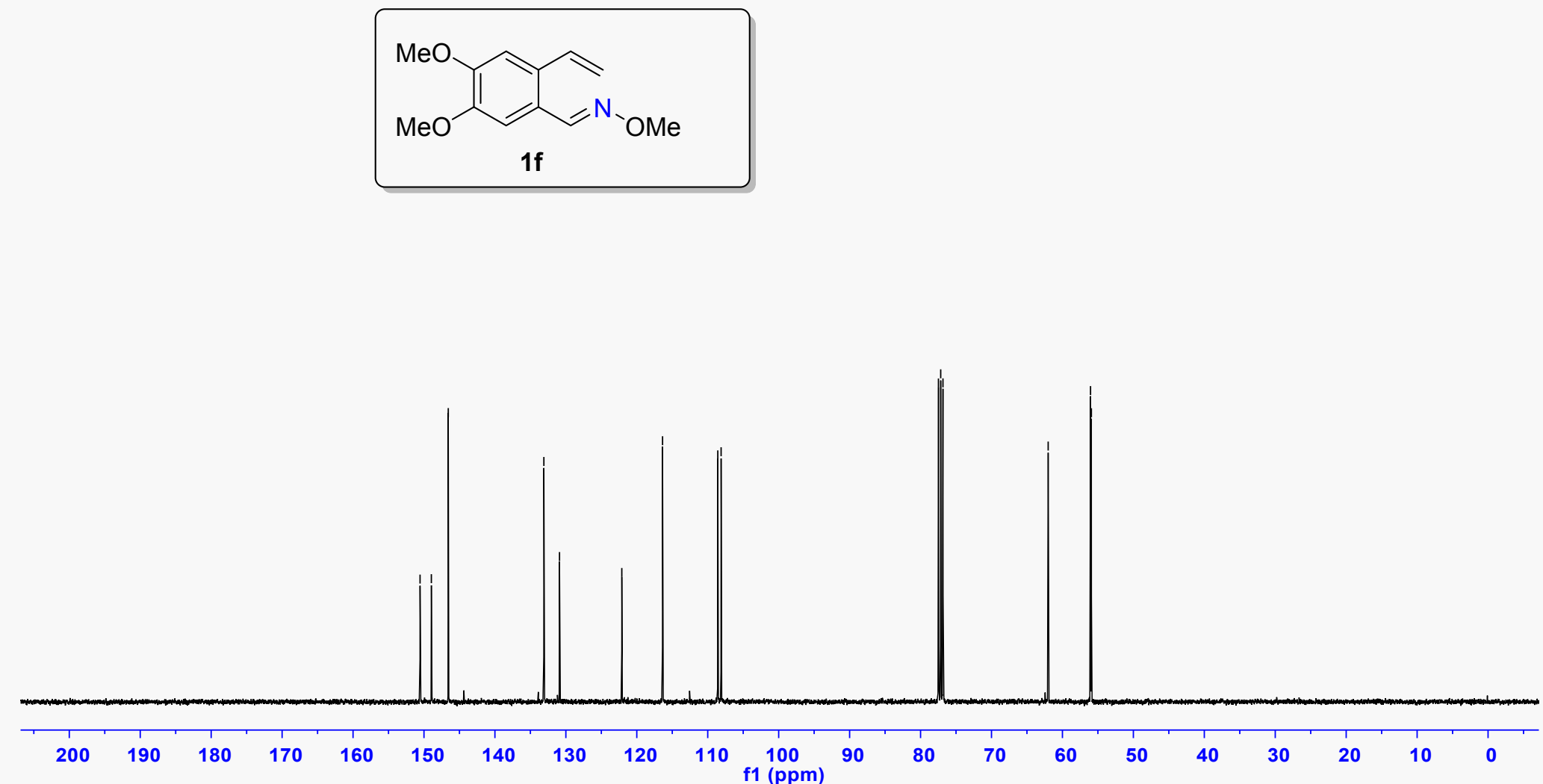
1g-H (400 MHz, CDCl3)

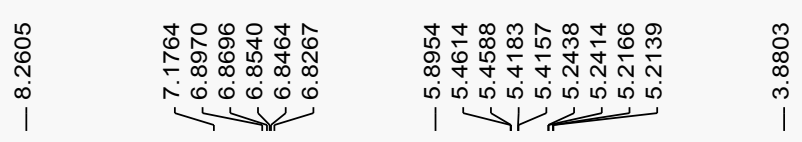

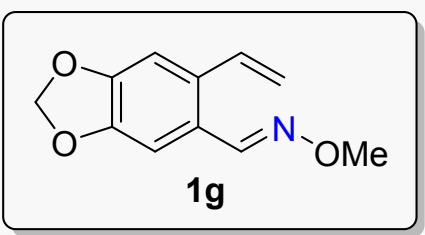

1g

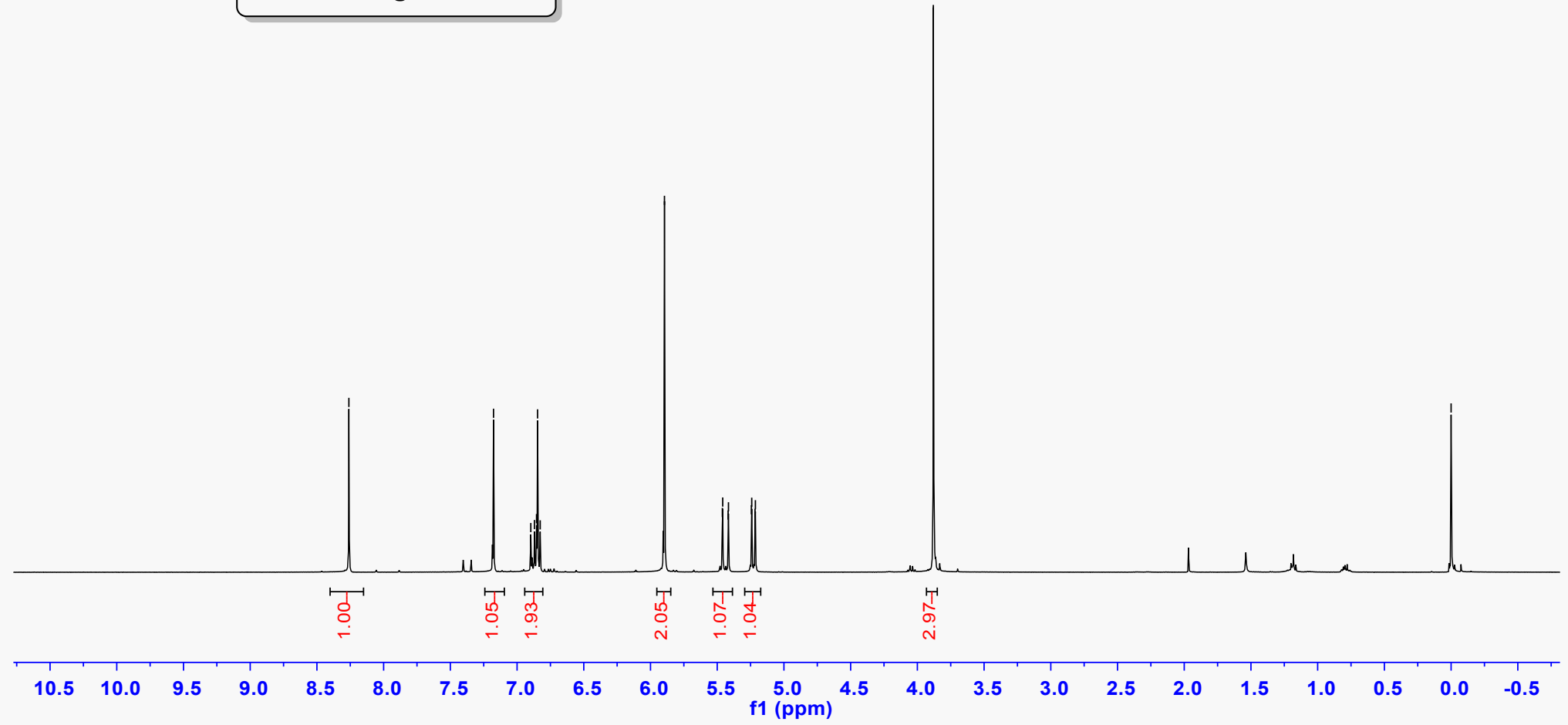



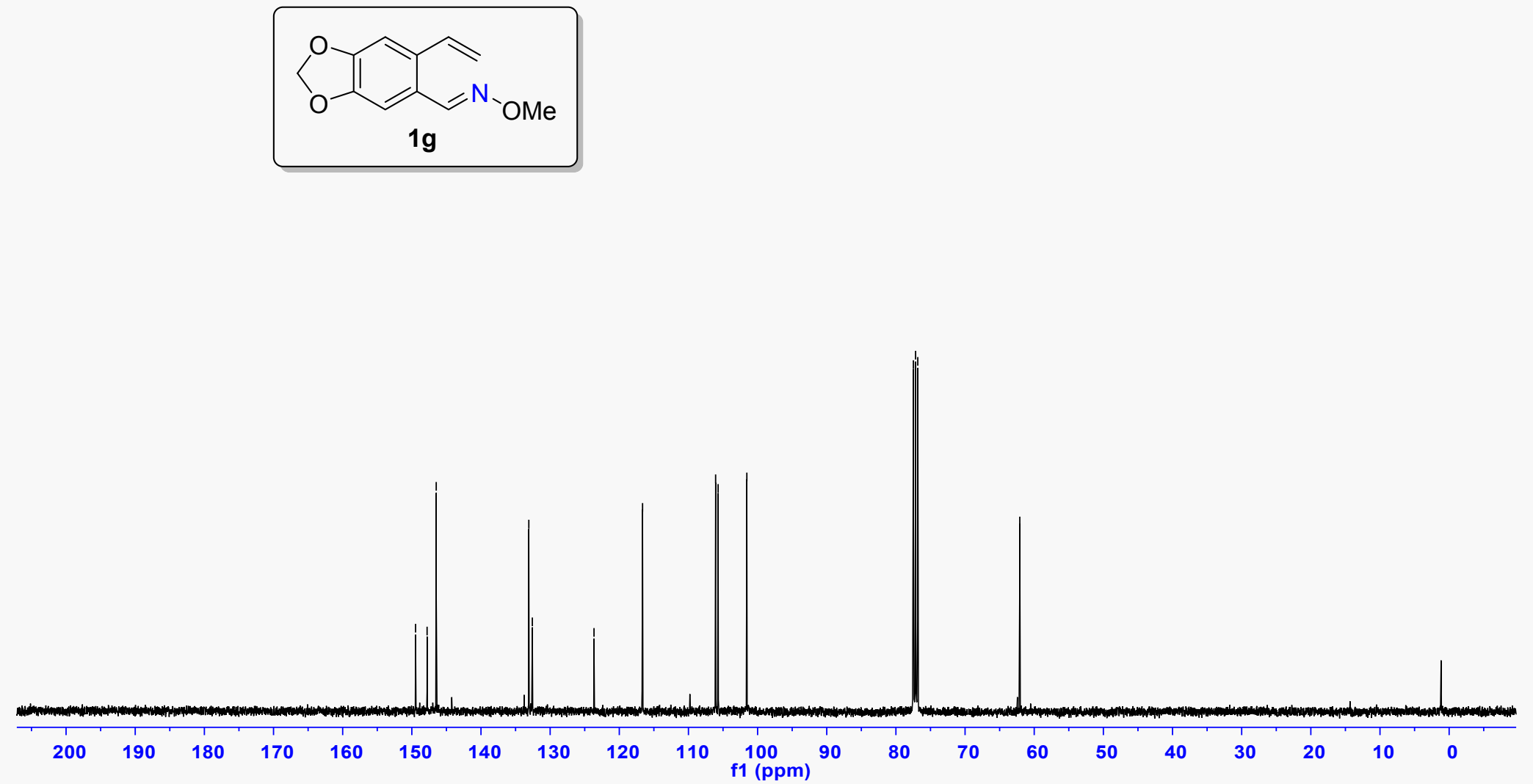
1m-H (400 MHz, CDCl3)
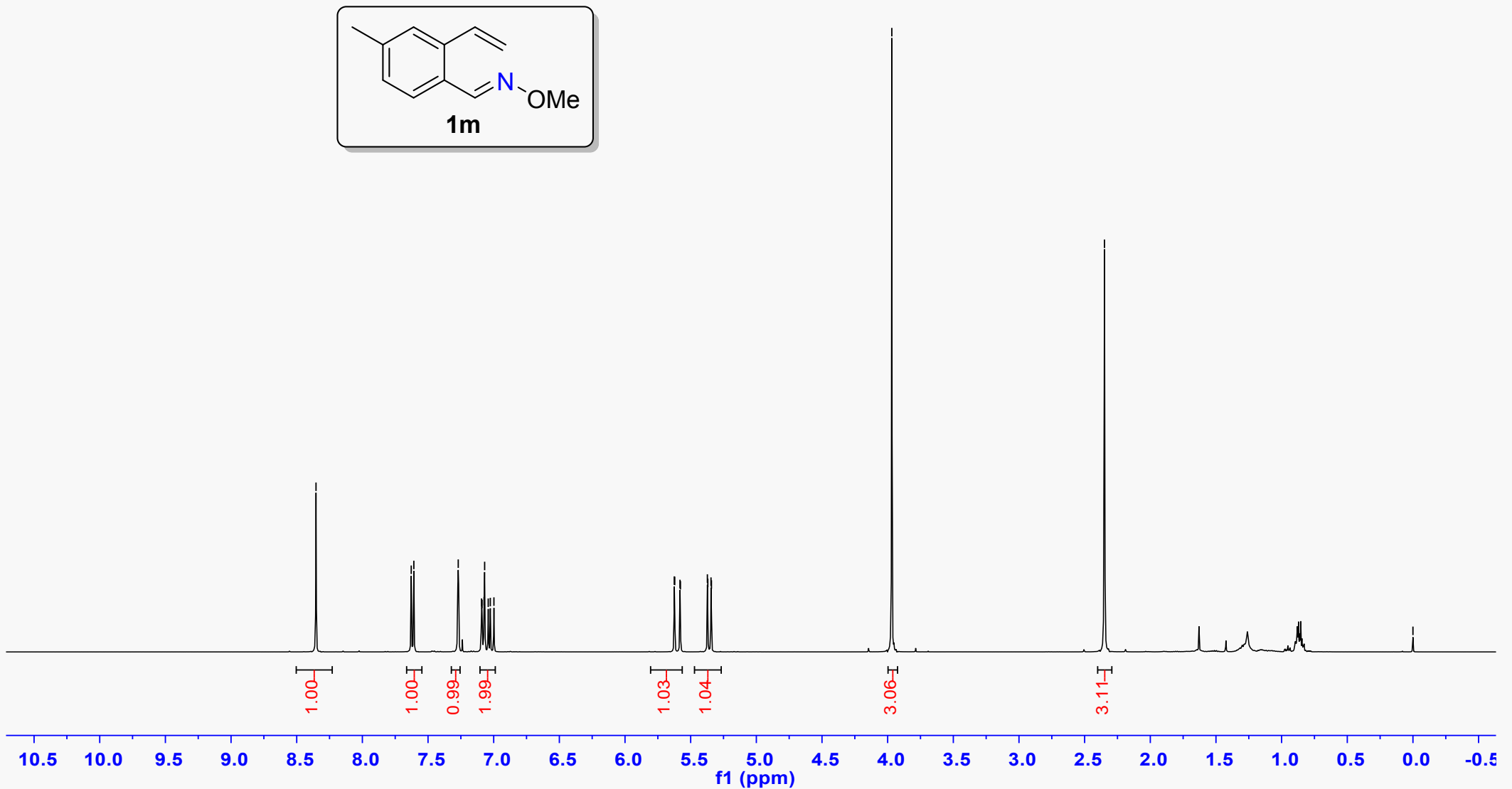
1m-C (100 MHz, CDCl3)
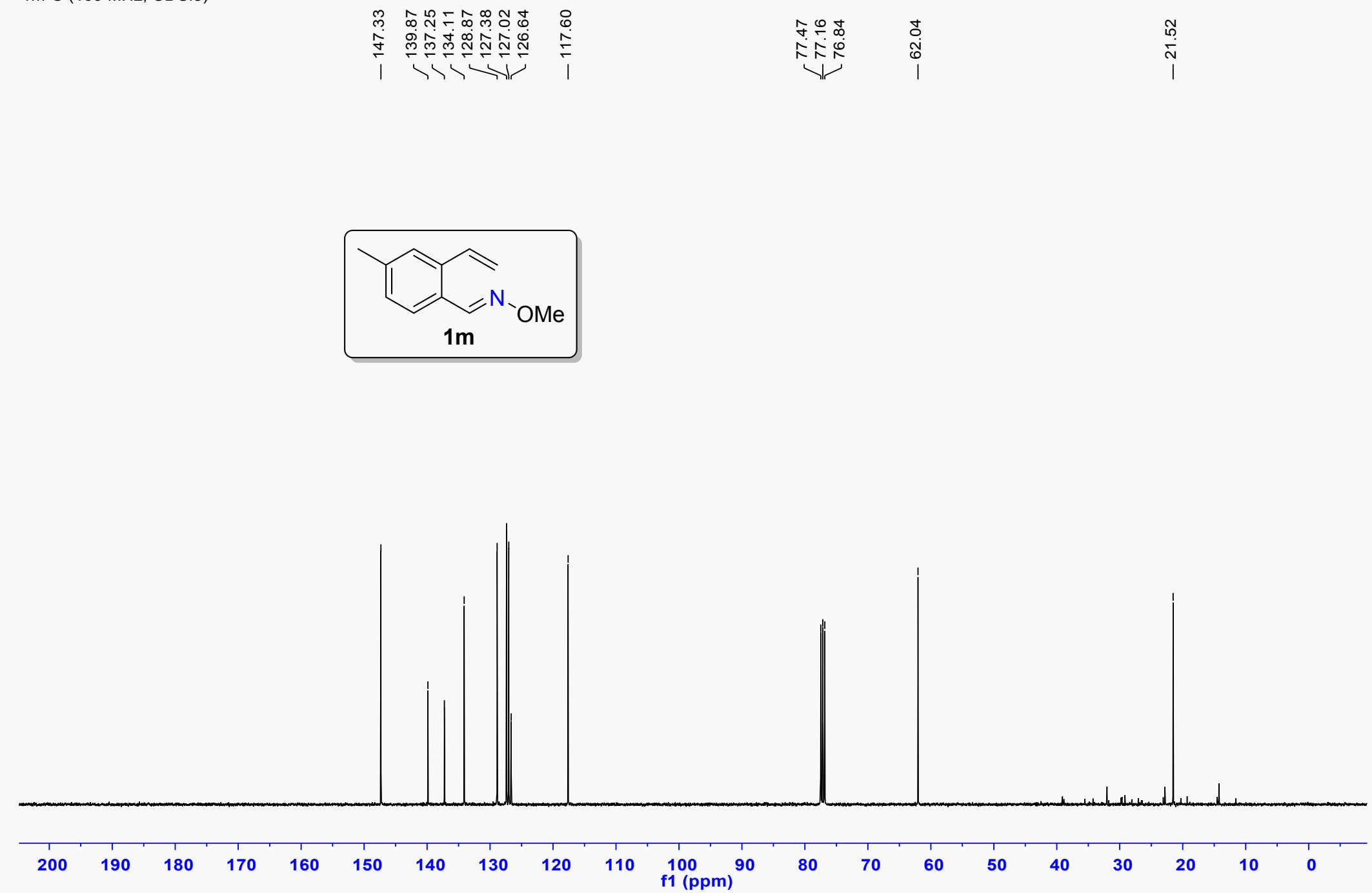

क人

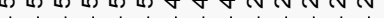
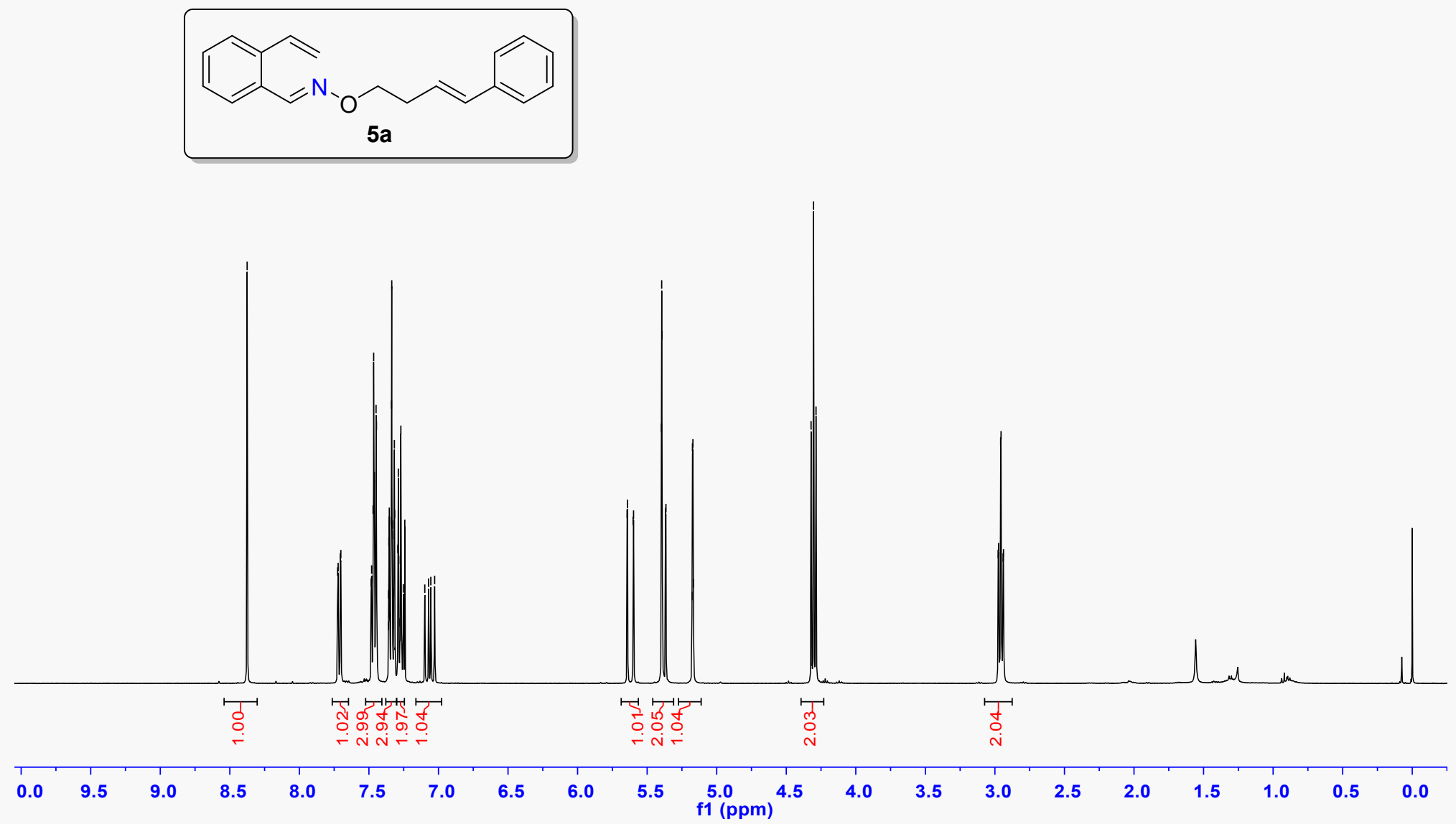

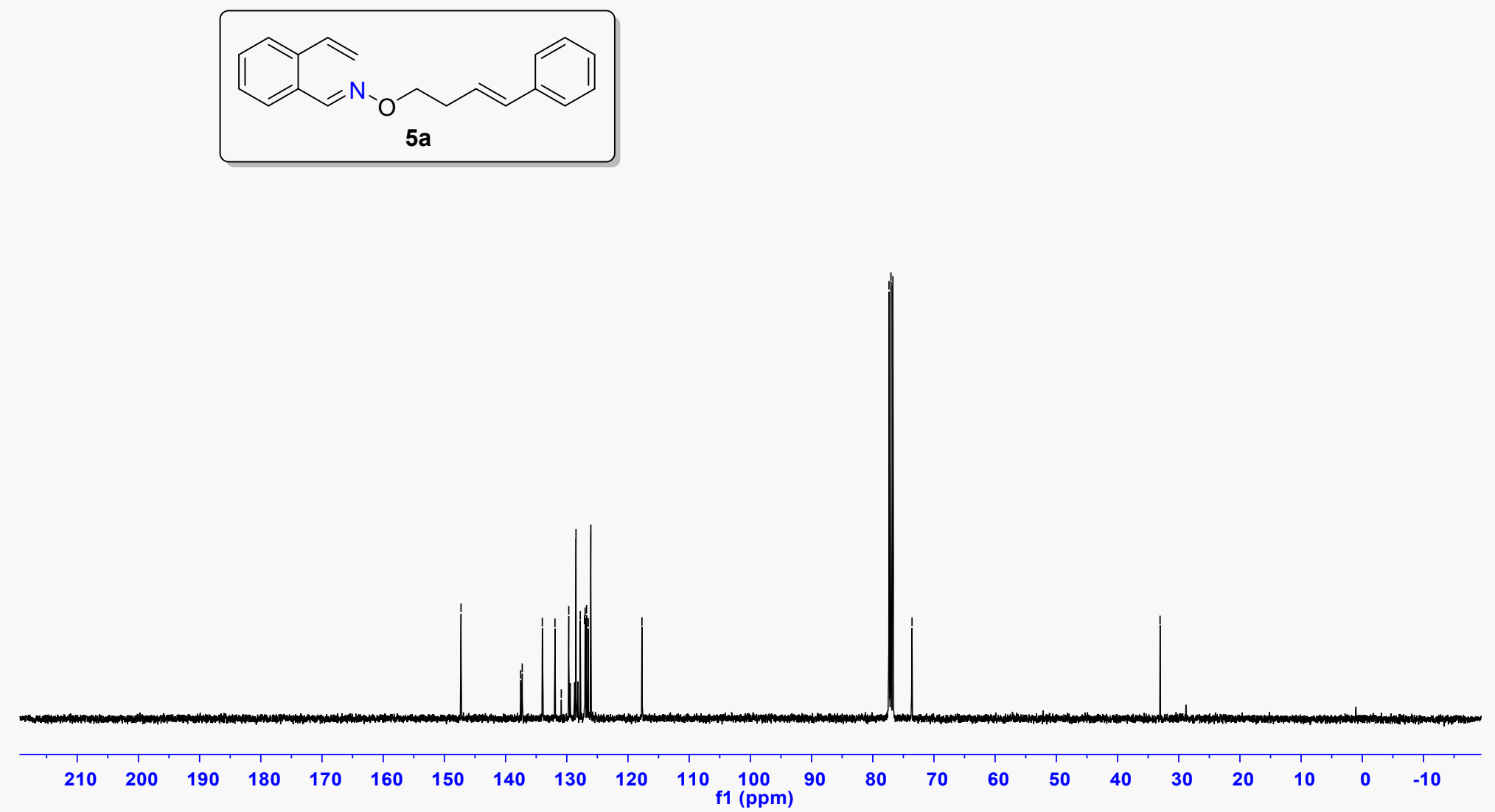
$5 \mathrm{~b}-\mathrm{H}(400 \mathrm{MHz}, \mathrm{CDCl} 3)$

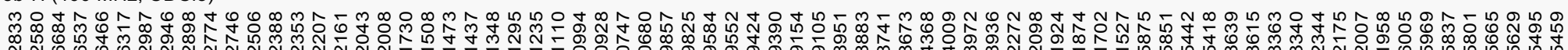

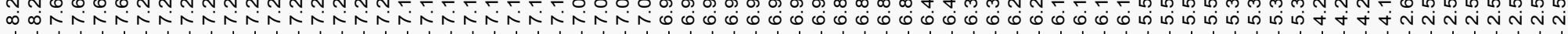
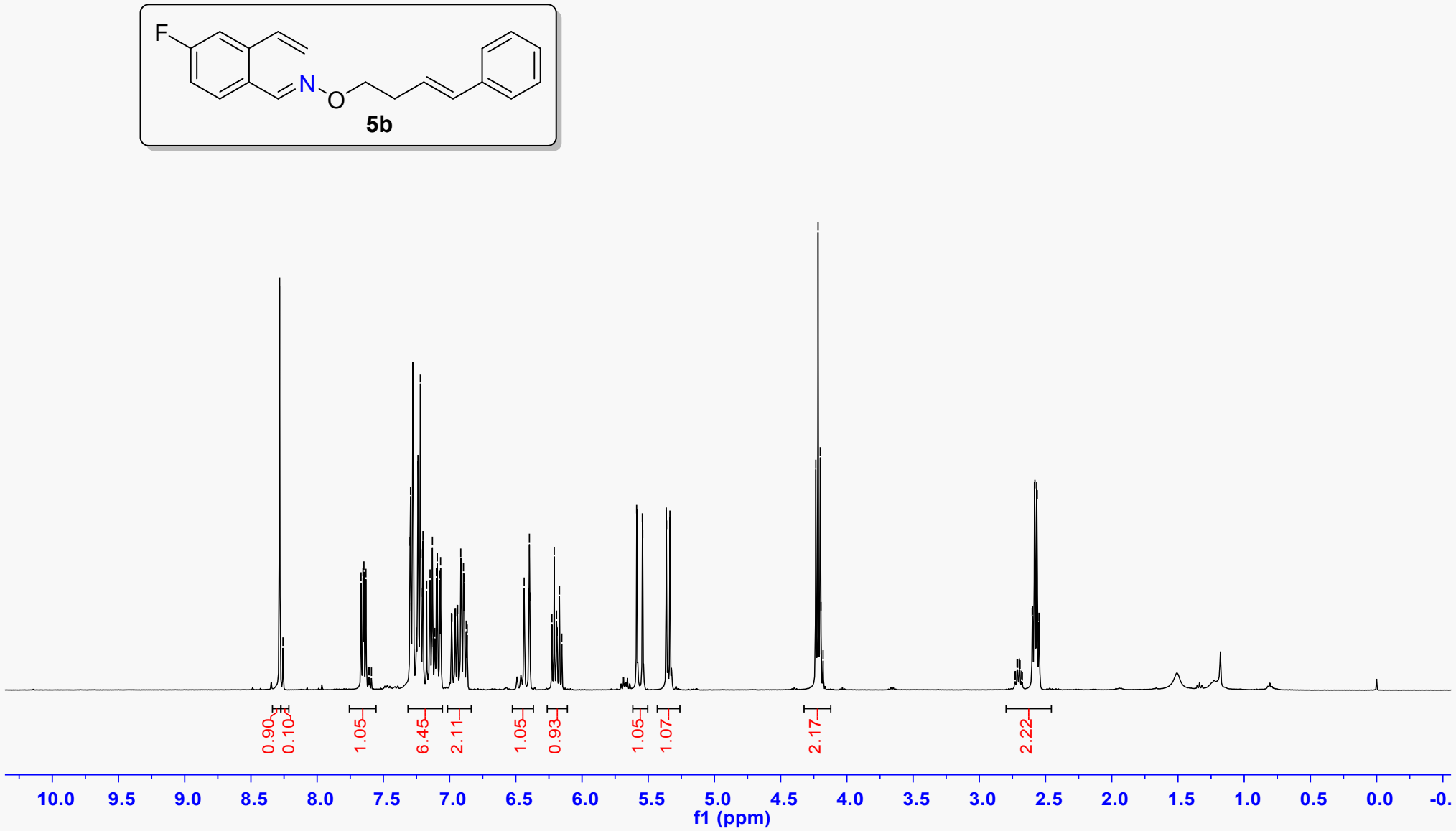

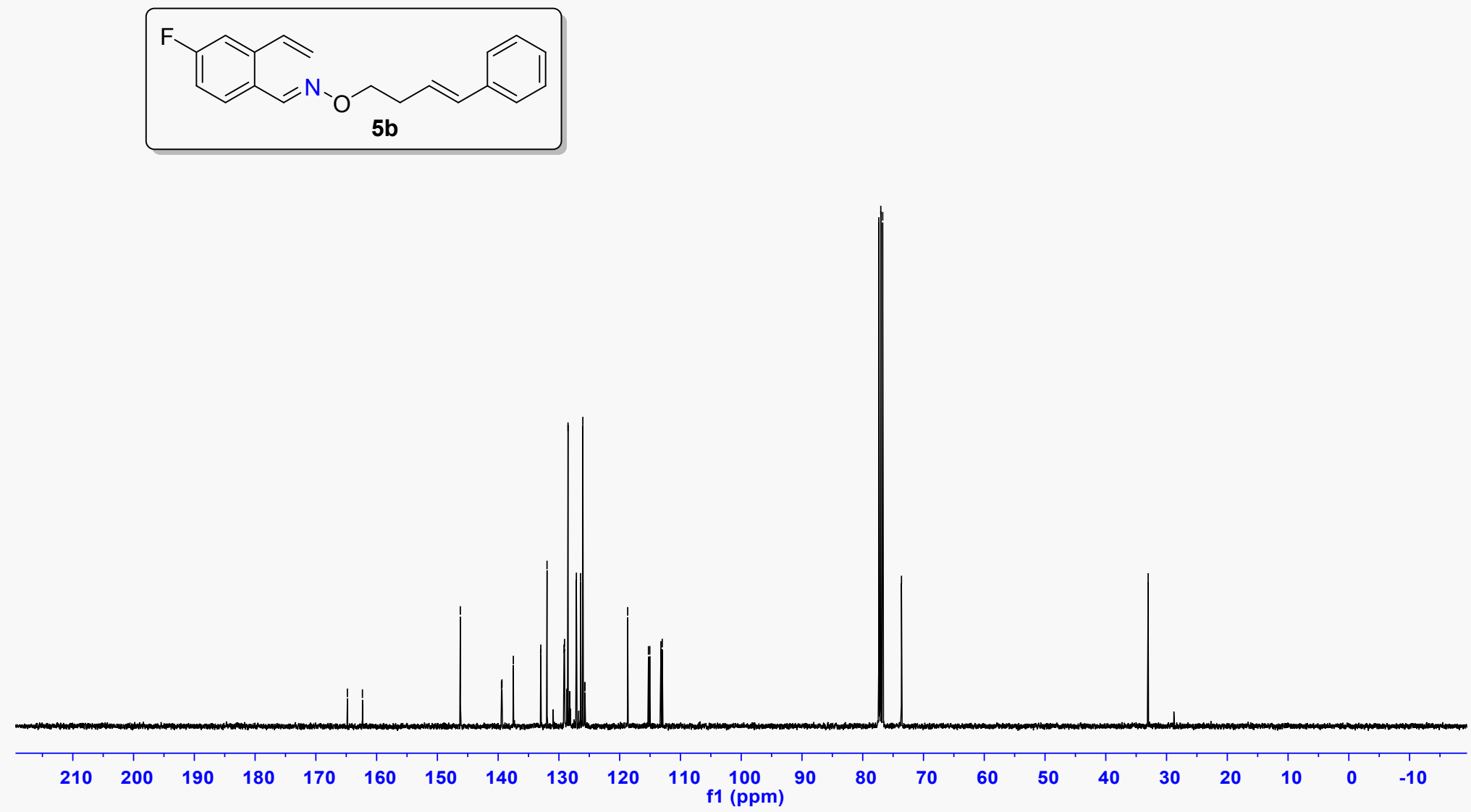

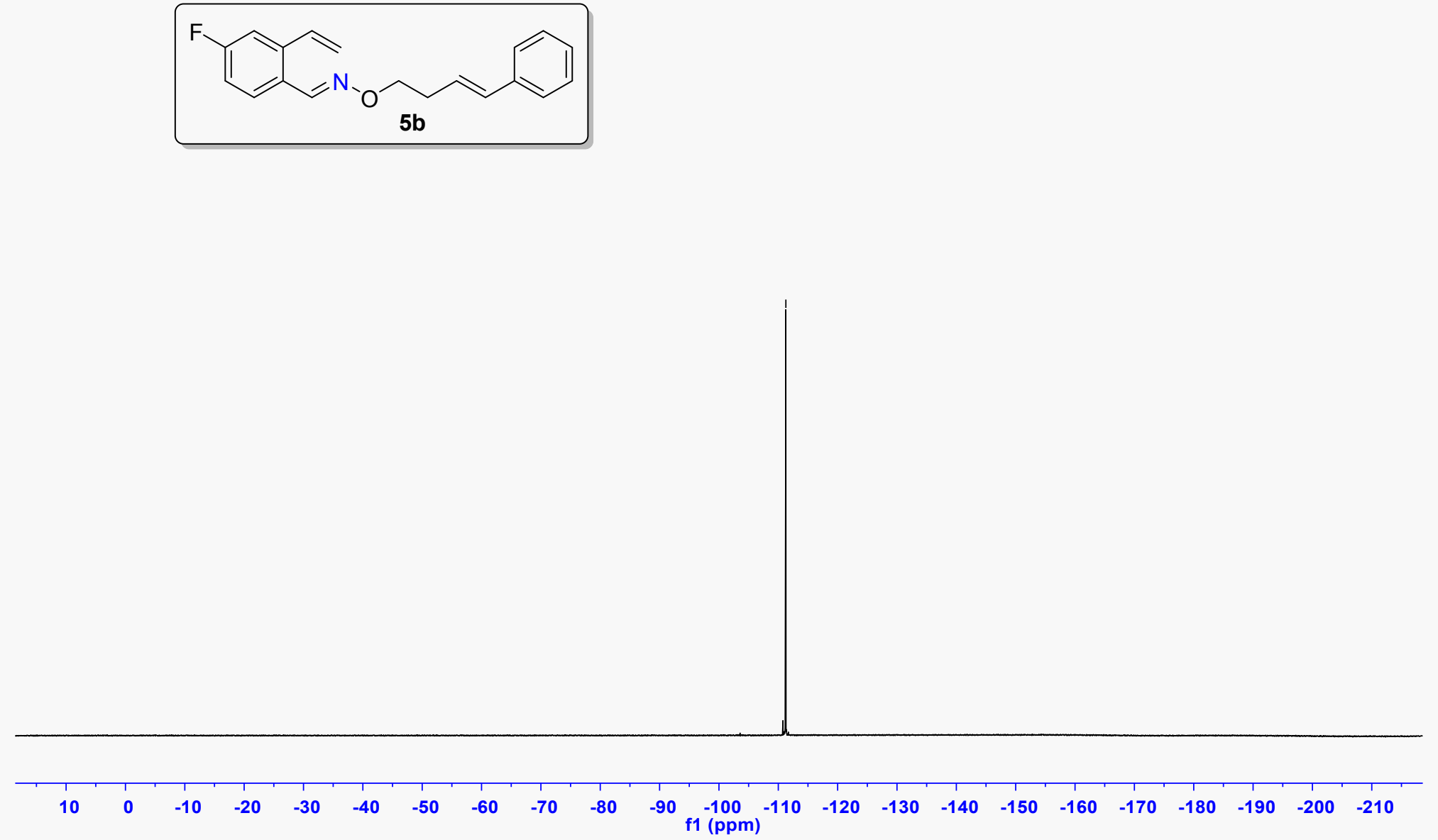
$5 \mathrm{C}-\mathrm{H}(400 \mathrm{MHz}, \mathrm{CDCl} 3)$

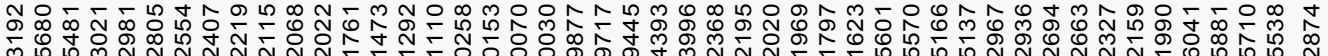

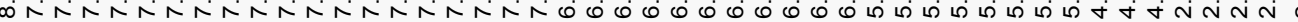
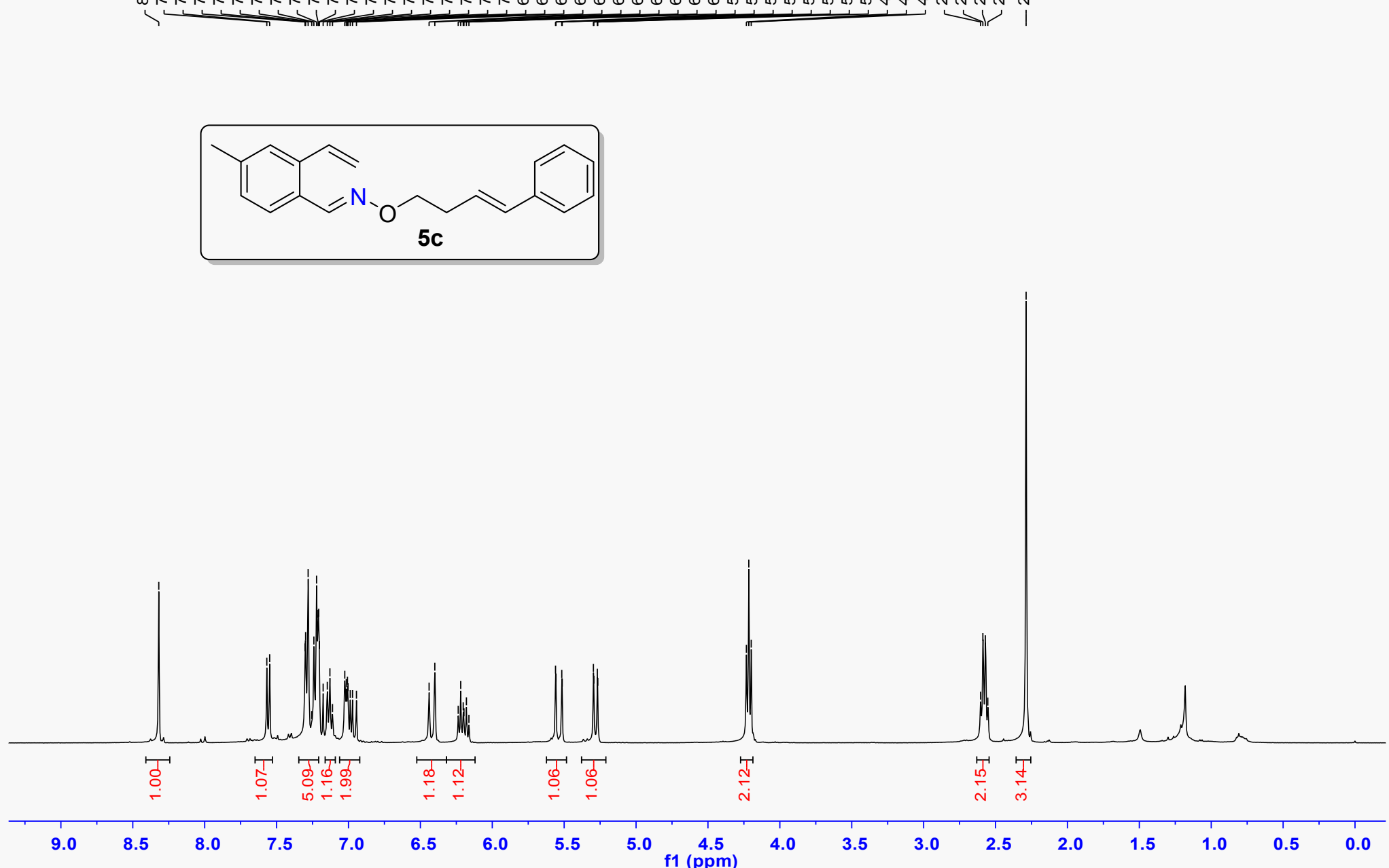
5c-C(100 MHz, CDCl3)
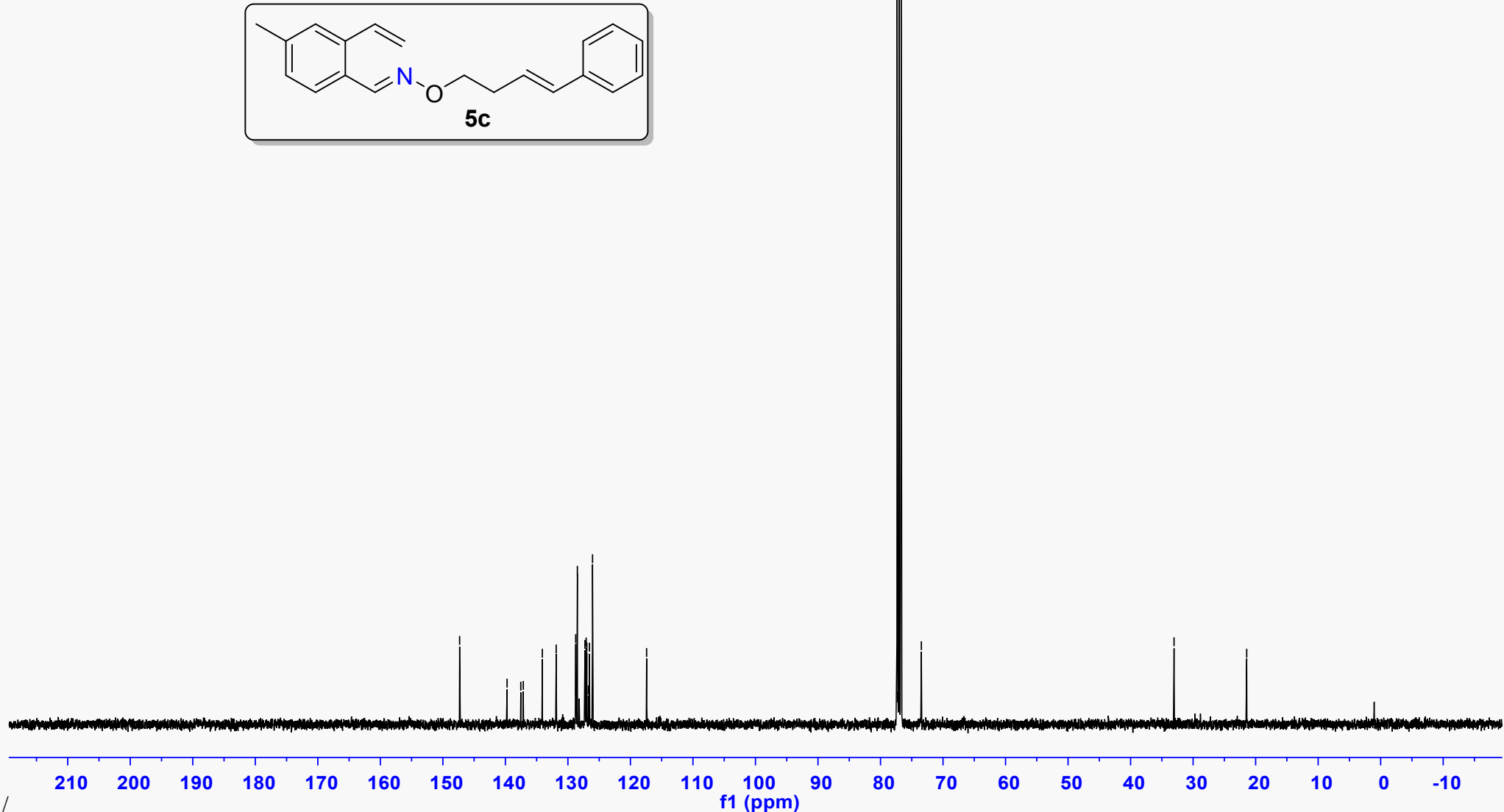
$5 \mathrm{~d}-\mathrm{H}(400 \mathrm{MHz}, \mathrm{CDCl} 3)$

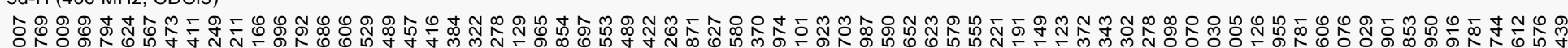

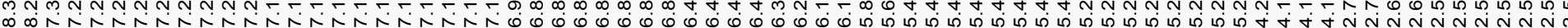
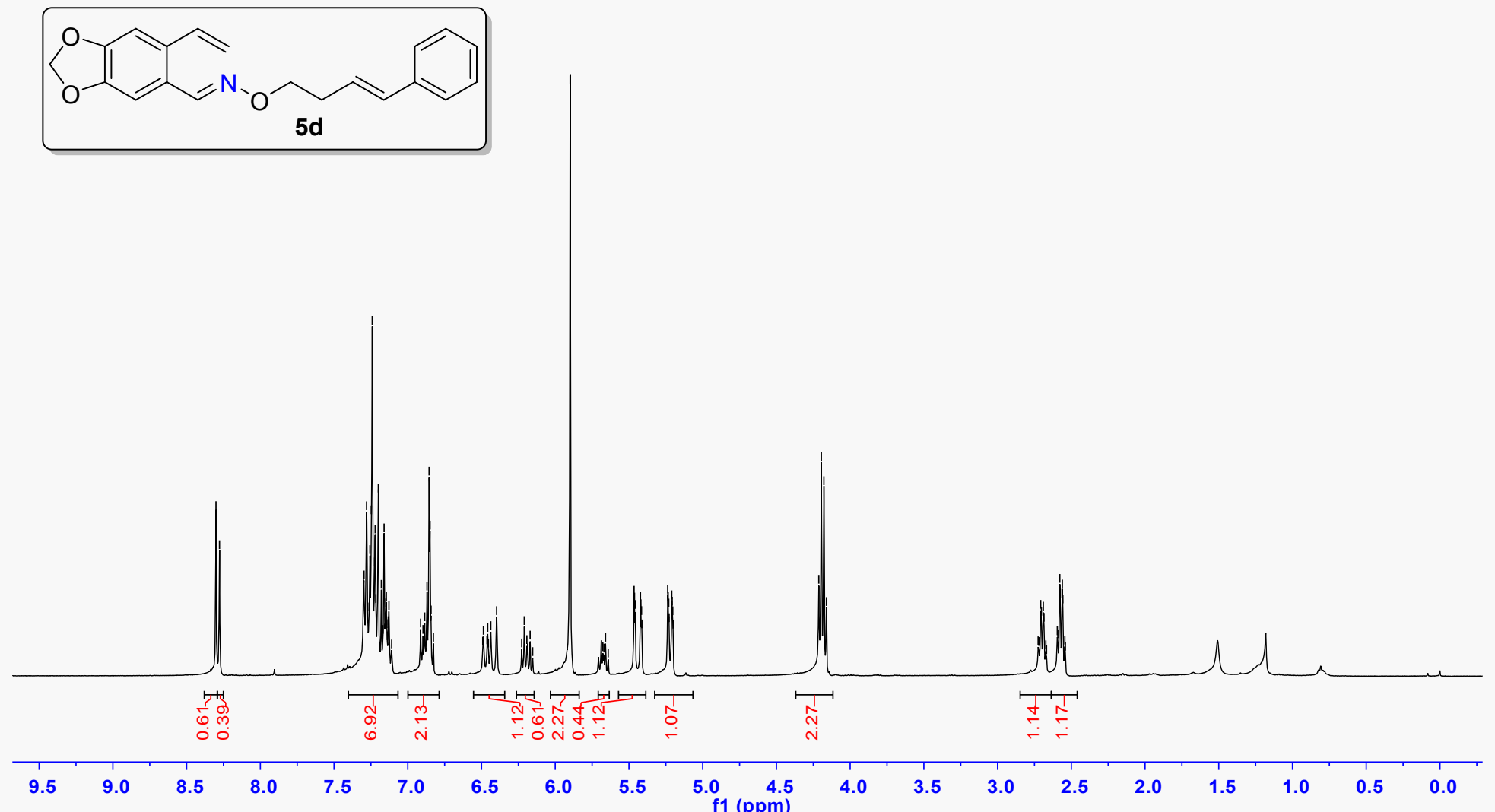

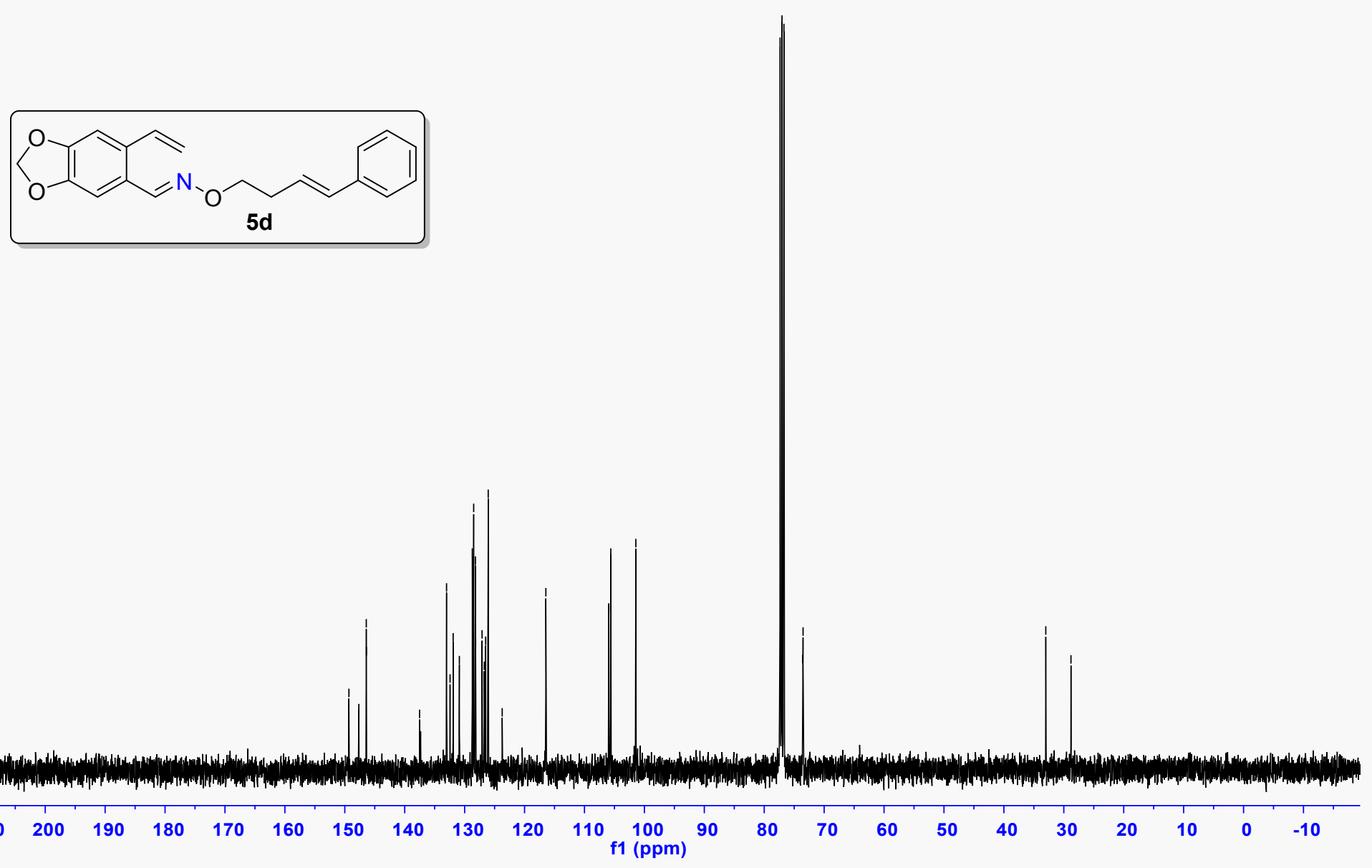

$\begin{array}{llllllllllll}210 & 200 & 190 & 180 & 170 & 160 & 150 & 140 & 130 & 120 & 110 \underset{\mathrm{f} 1}{(\mathrm{ppm})} & \begin{array}{l}100 \\ 90\end{array}\end{array}$ 
$5 \mathrm{e}-\mathrm{H}(400 \mathrm{MHz}, \mathrm{CDCl} 3)$

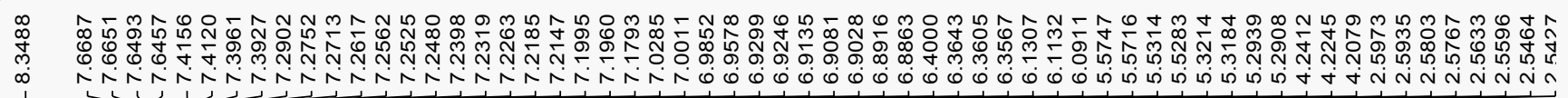
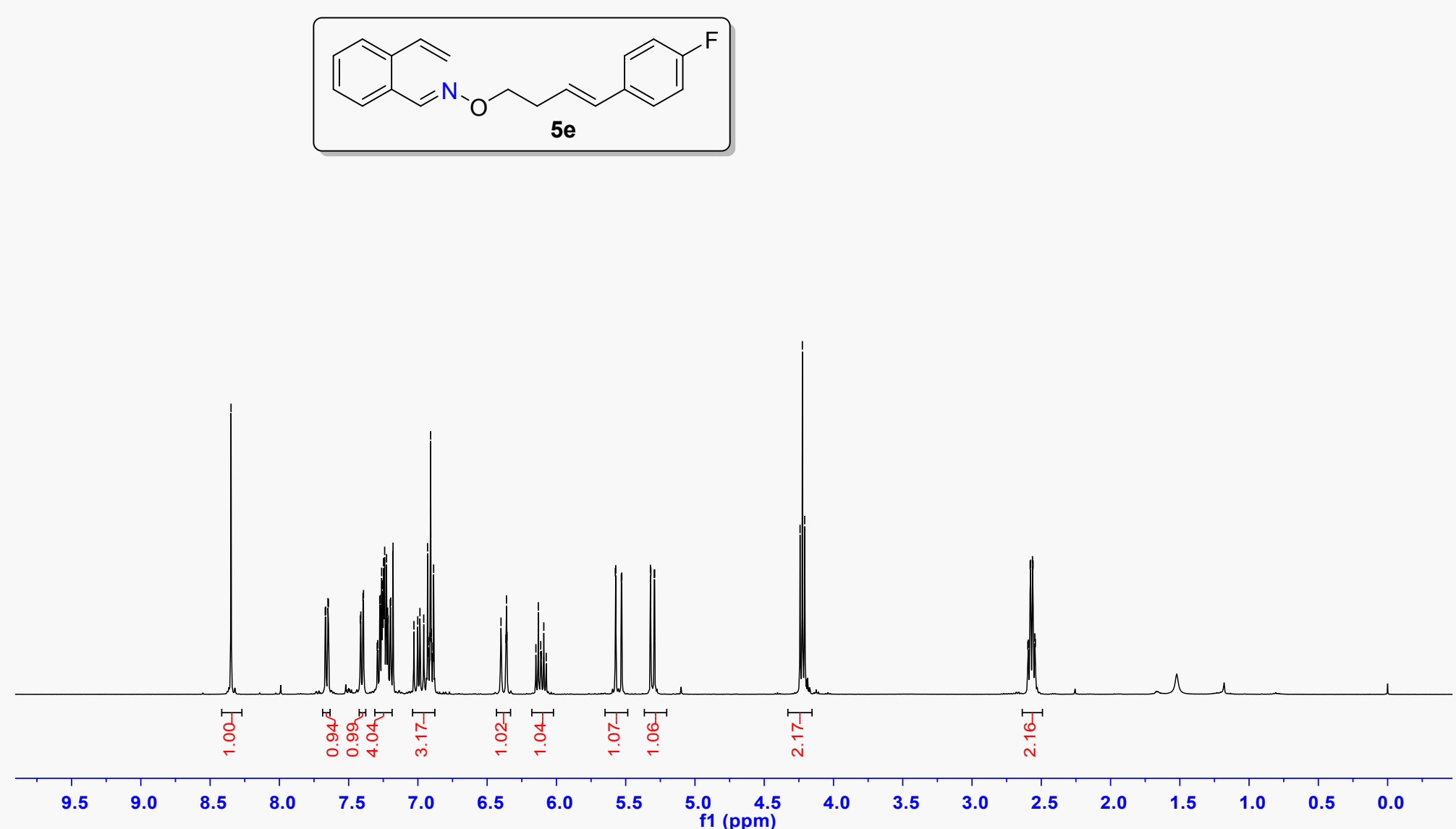
5e-C (100 MHz, CDCl3)

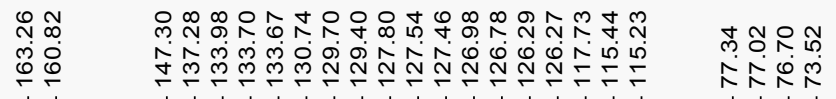

$\stackrel{\infty}{\stackrel{\infty}{\sim}}$

प

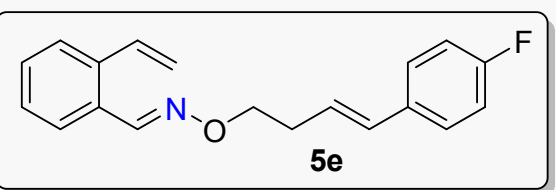

$5 e$

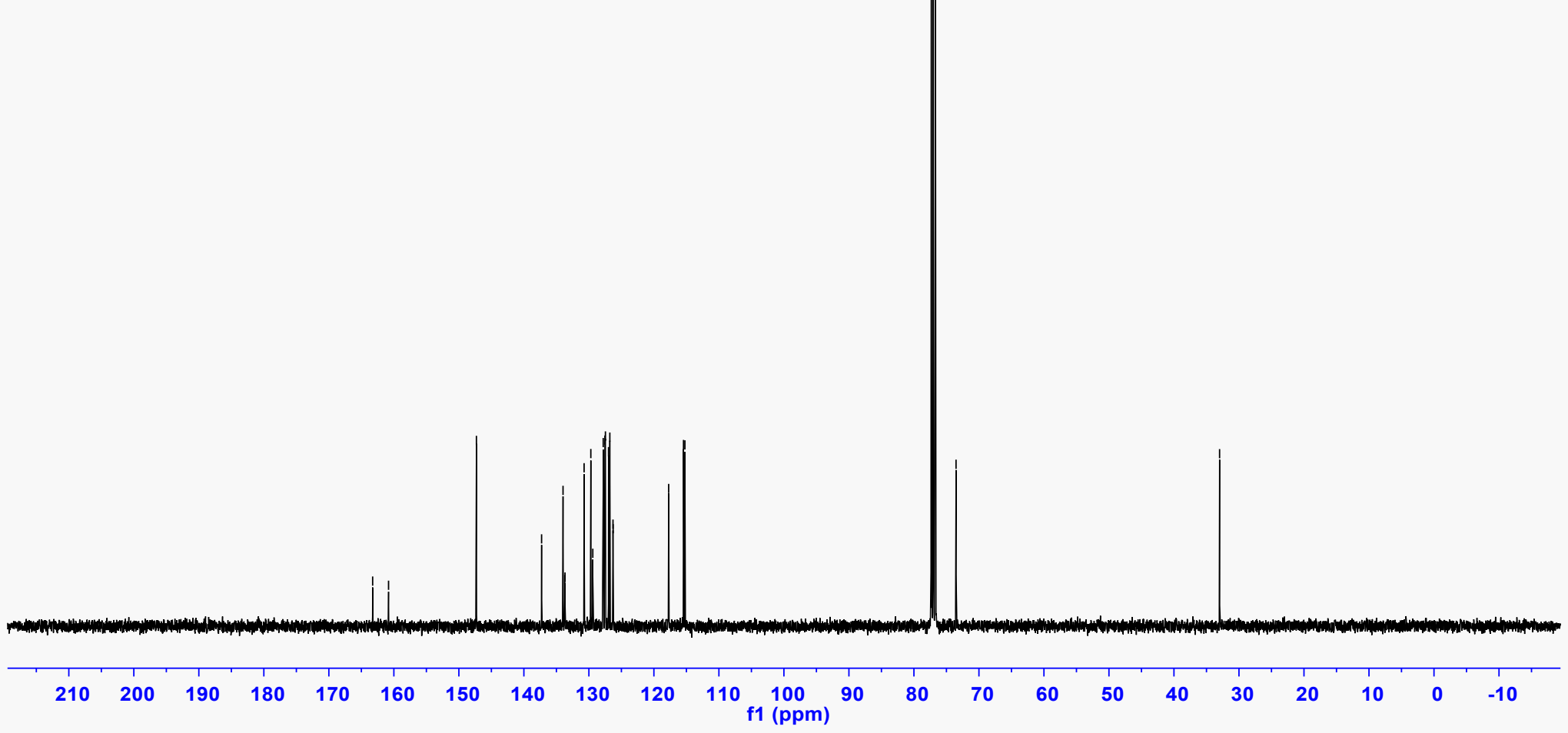


5e-F (376 MHz, CDCl3)
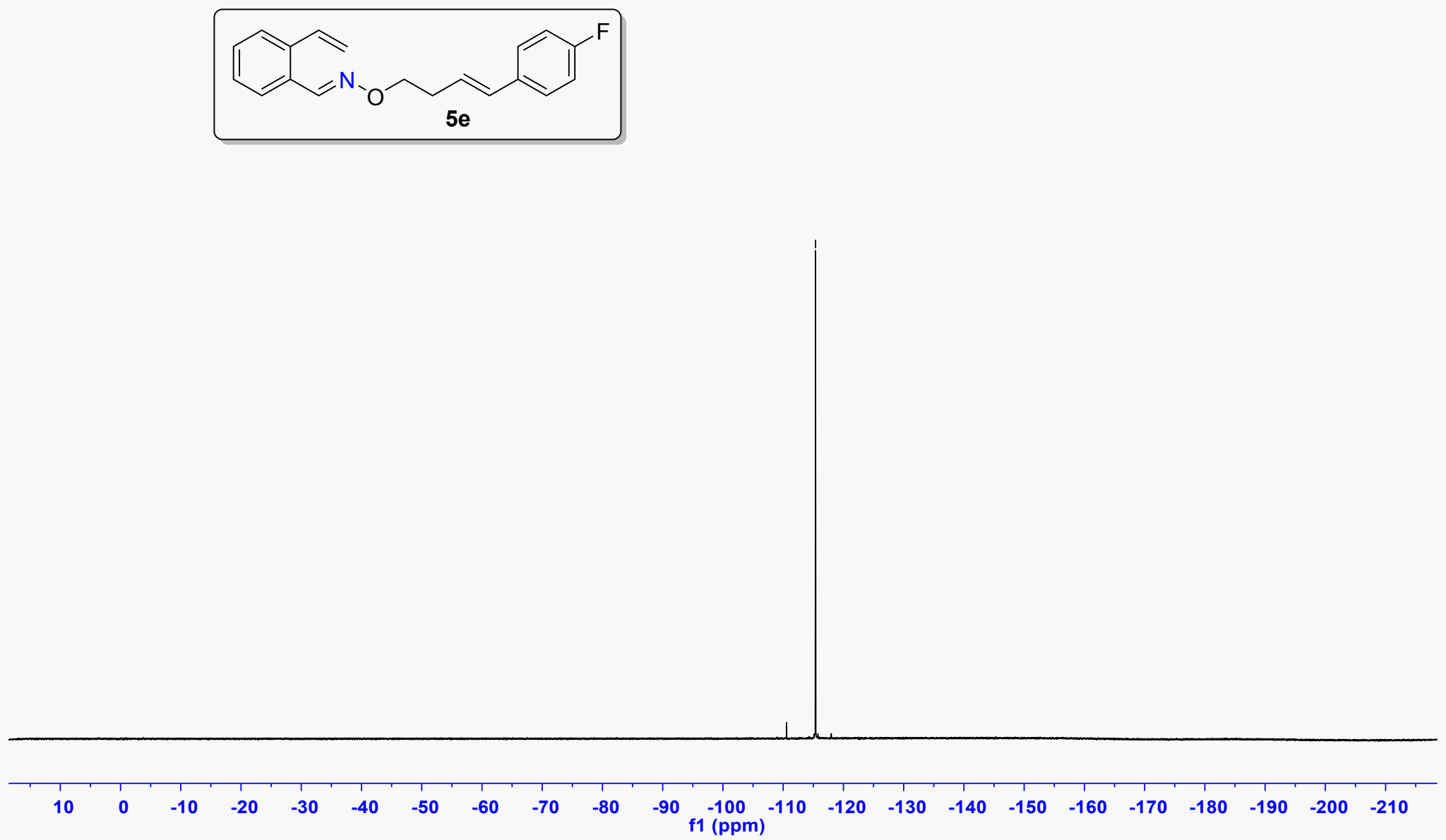
5f-H (400 MHz, CDCl3)

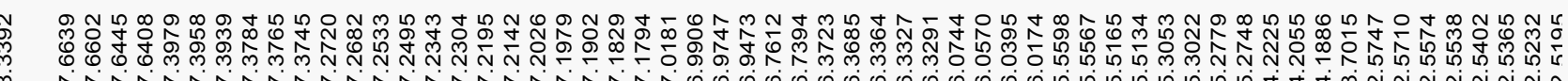

1
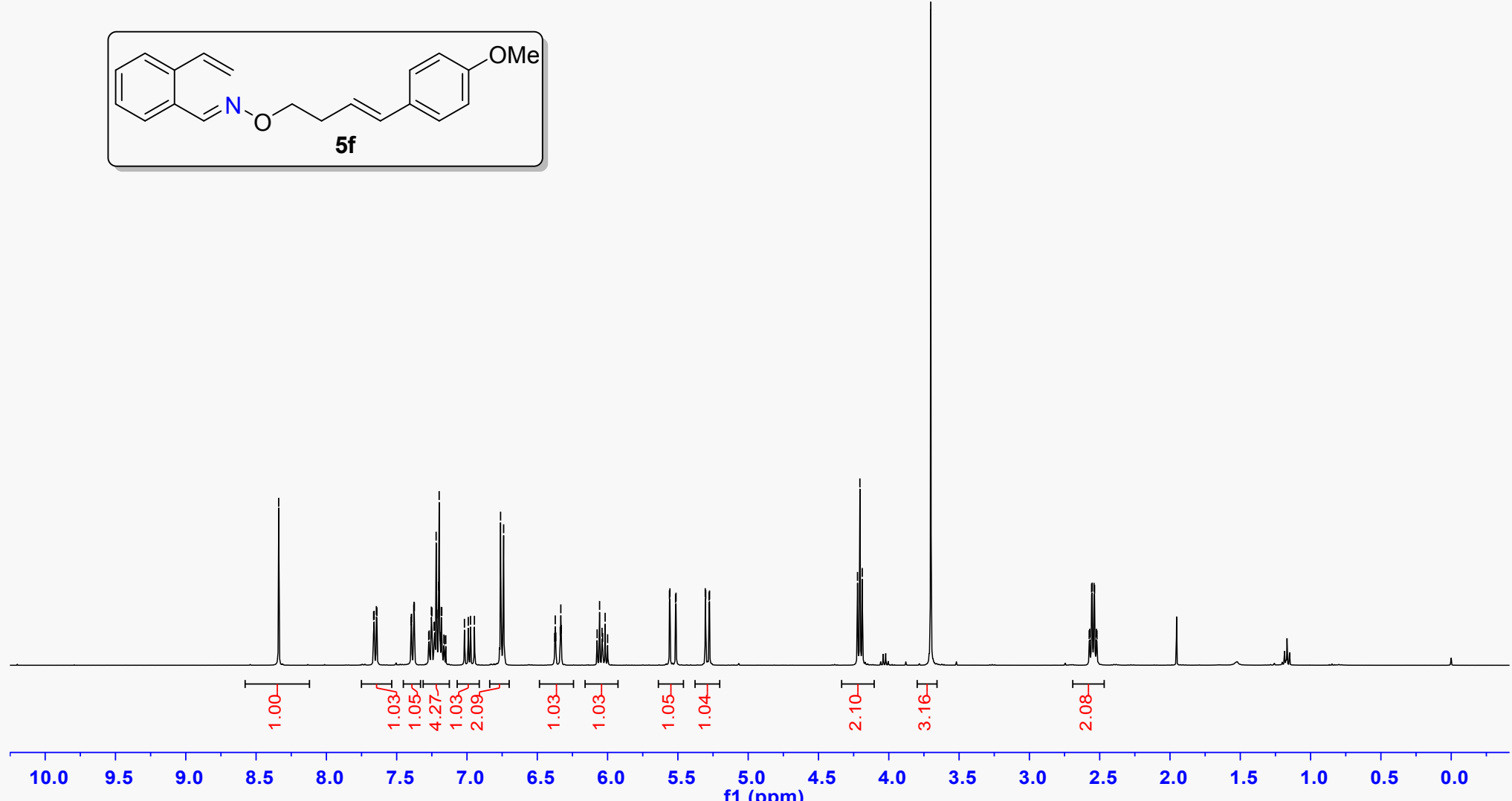
5f-C(100 MHz, CDCl3)

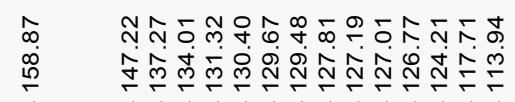

药定

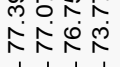

ָָ

$\stackrel{\substack{0 \\ m}}{1}$
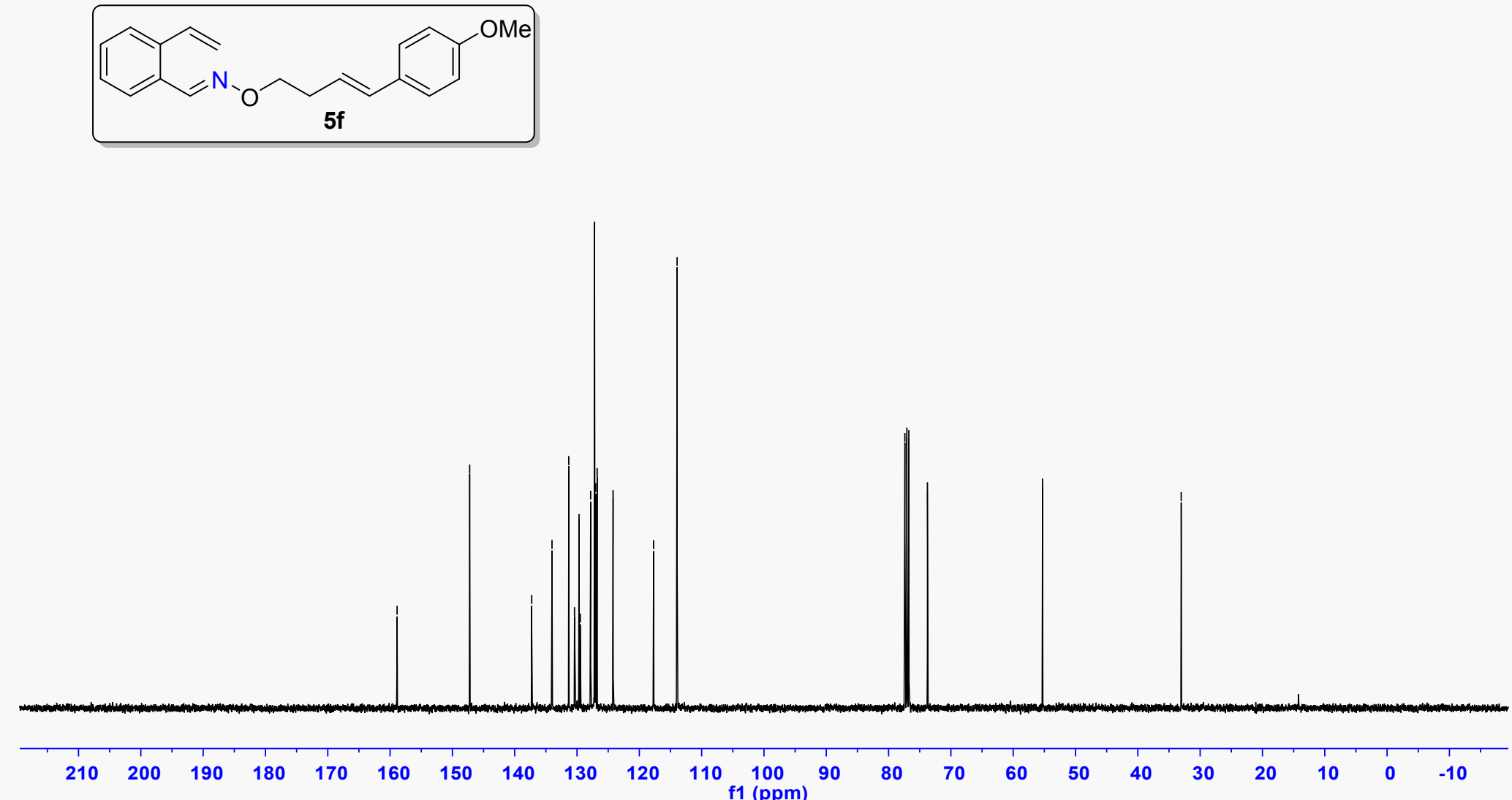
$\mathrm{S} 2-\mathrm{H}(400 \mathrm{MHz}, \mathrm{CDCl} 3)$

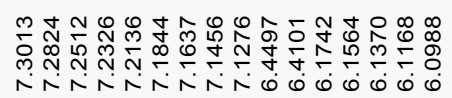

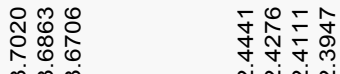

ले ले

$\underbrace{-4 \pi}$

$$
\overbrace{\mathbf{S 2}}^{\mathrm{OH}}
$$

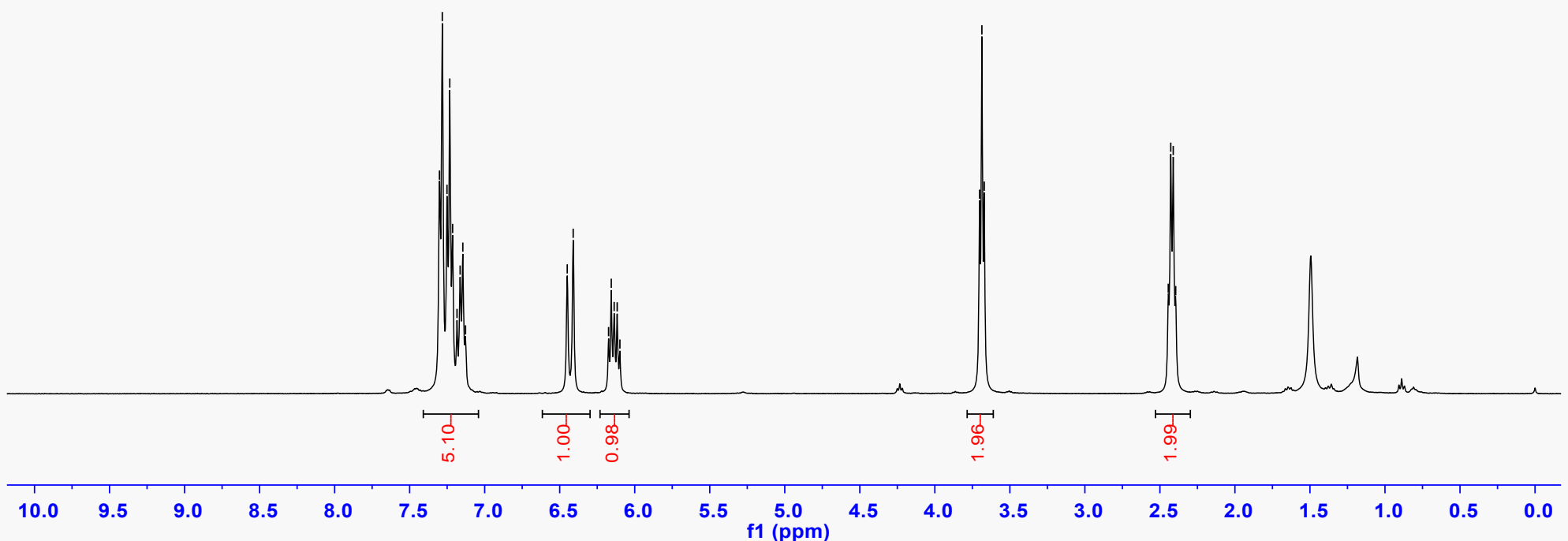


S2-C (100MHz,CDCl3)

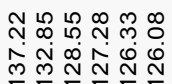

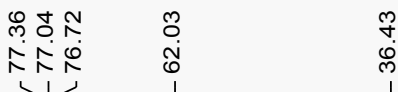

रे
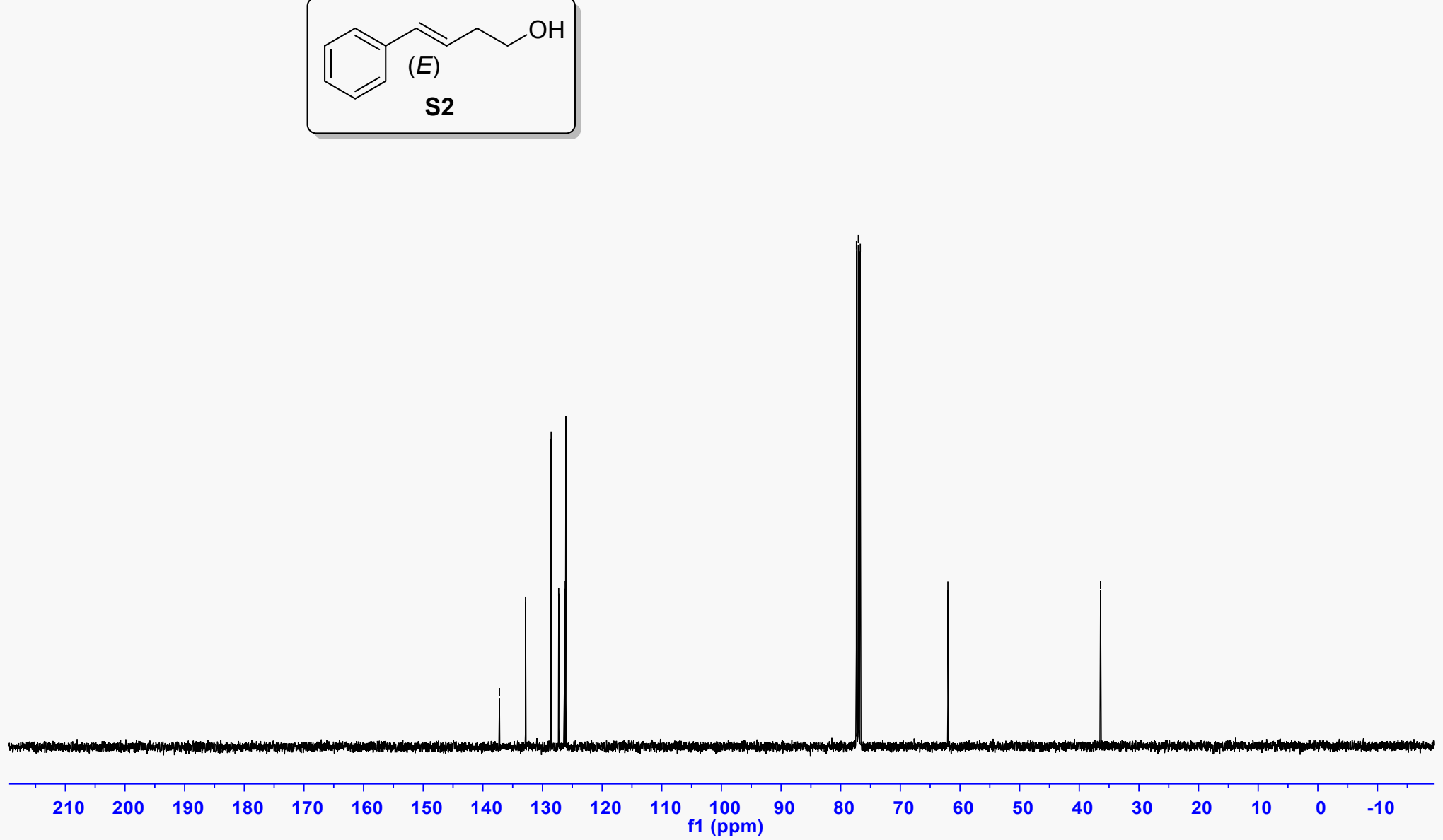

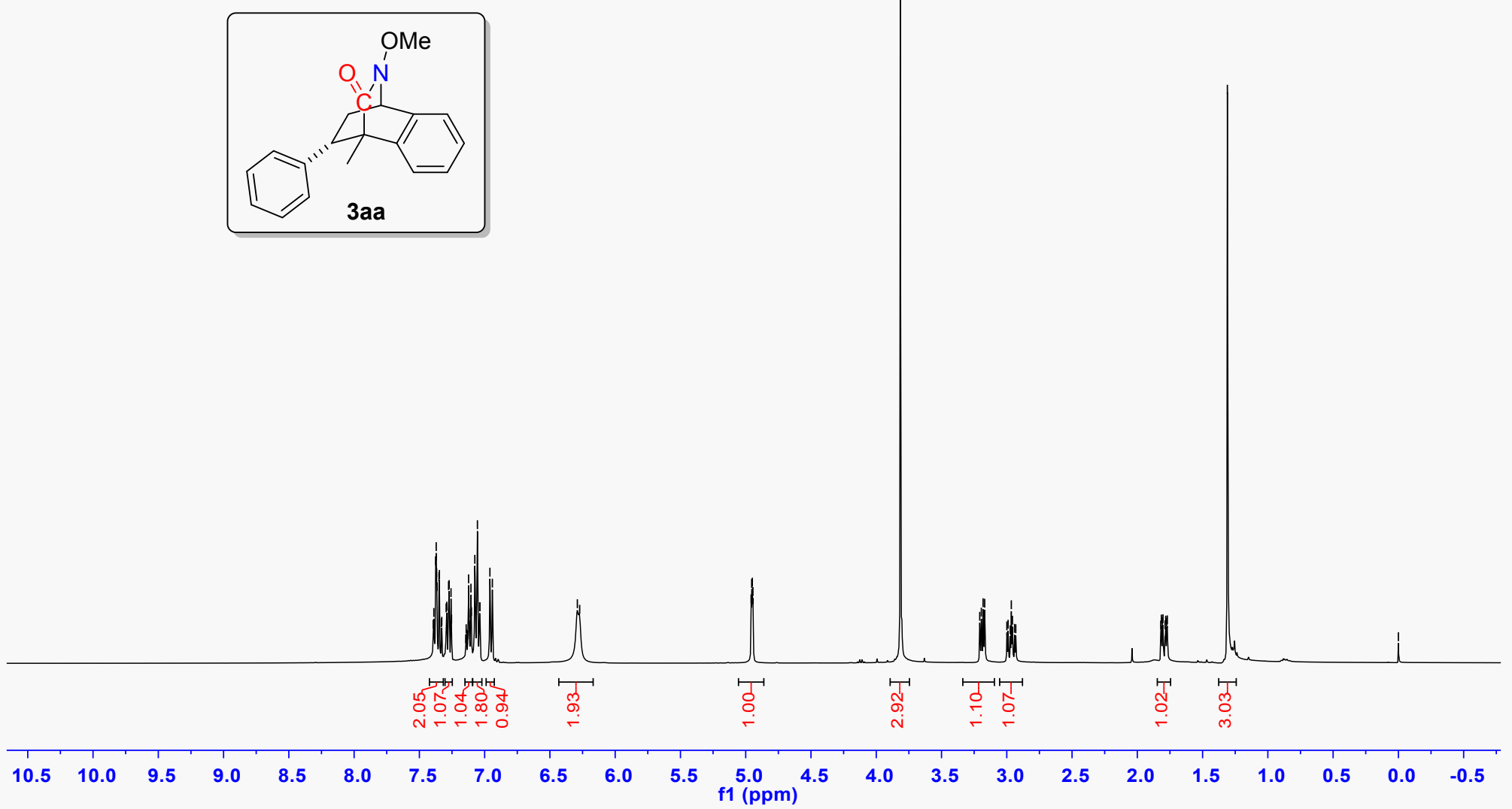
3аa-C (100 MHz, $\mathrm{CDCl} 3)$

$$
\text { I }
$$

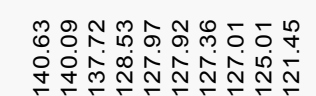

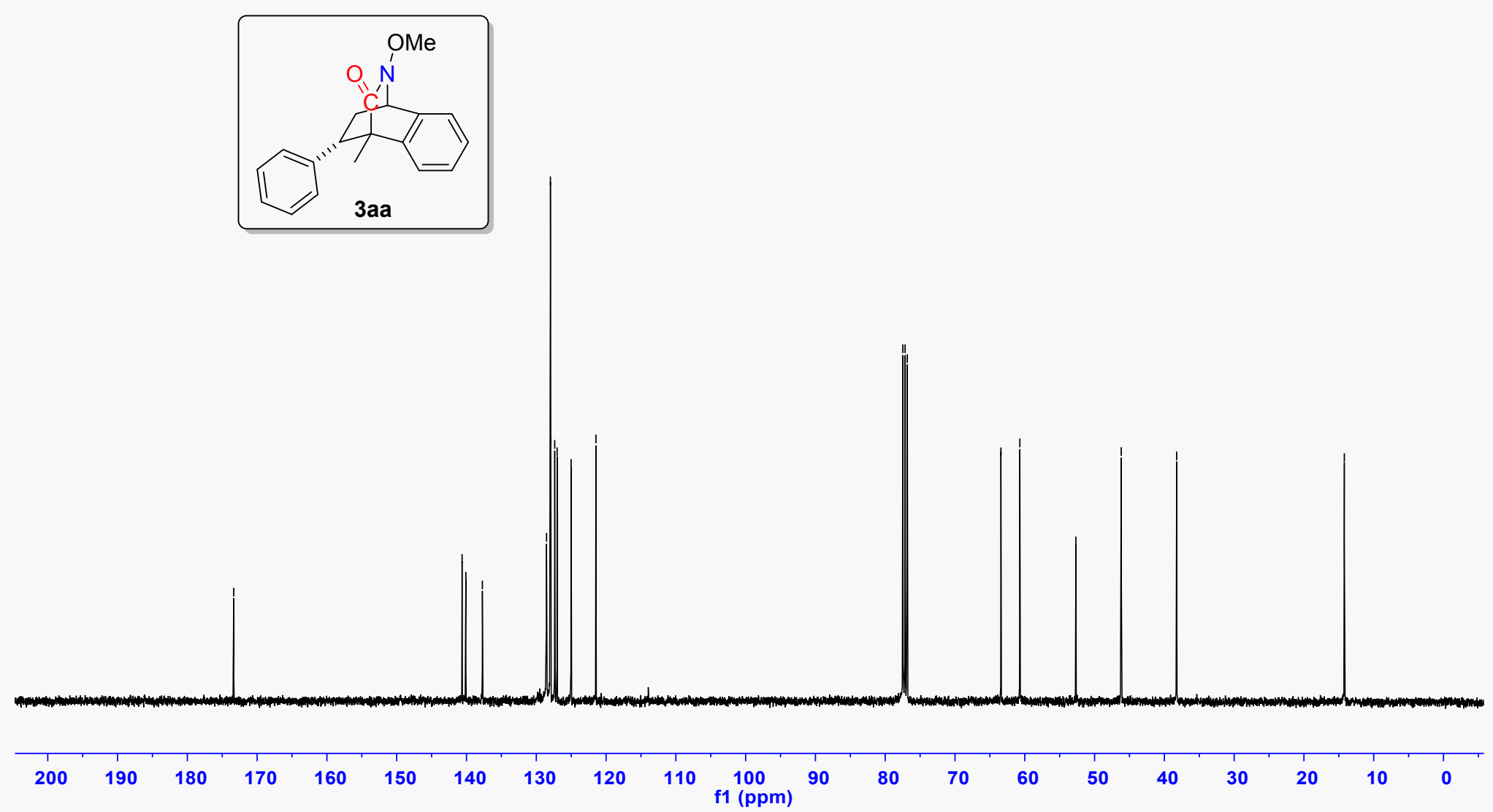




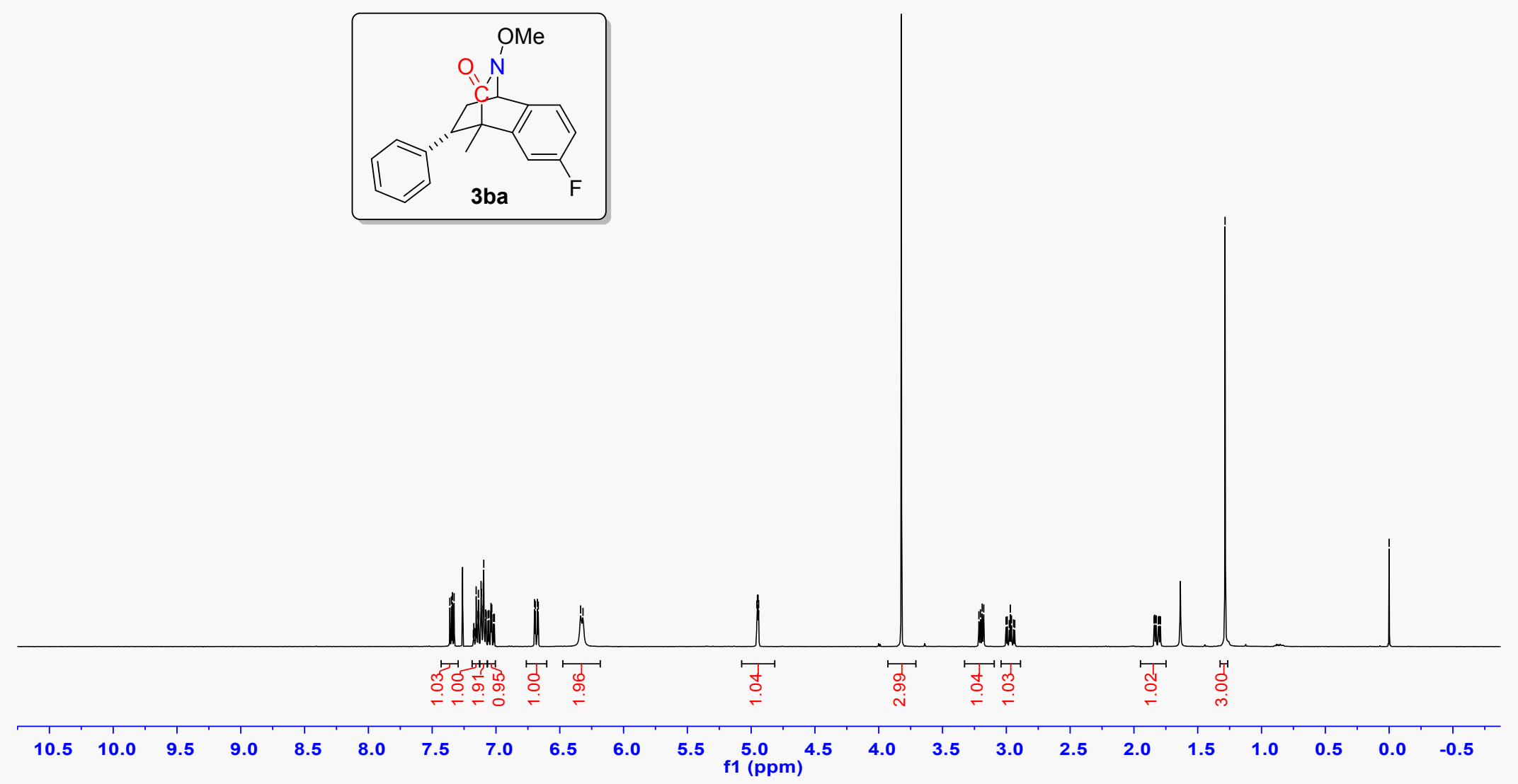


3ba-C (100 MHz, CDCl3)

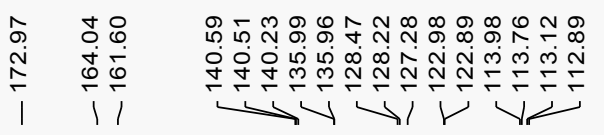

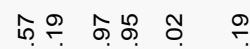

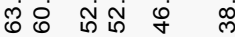
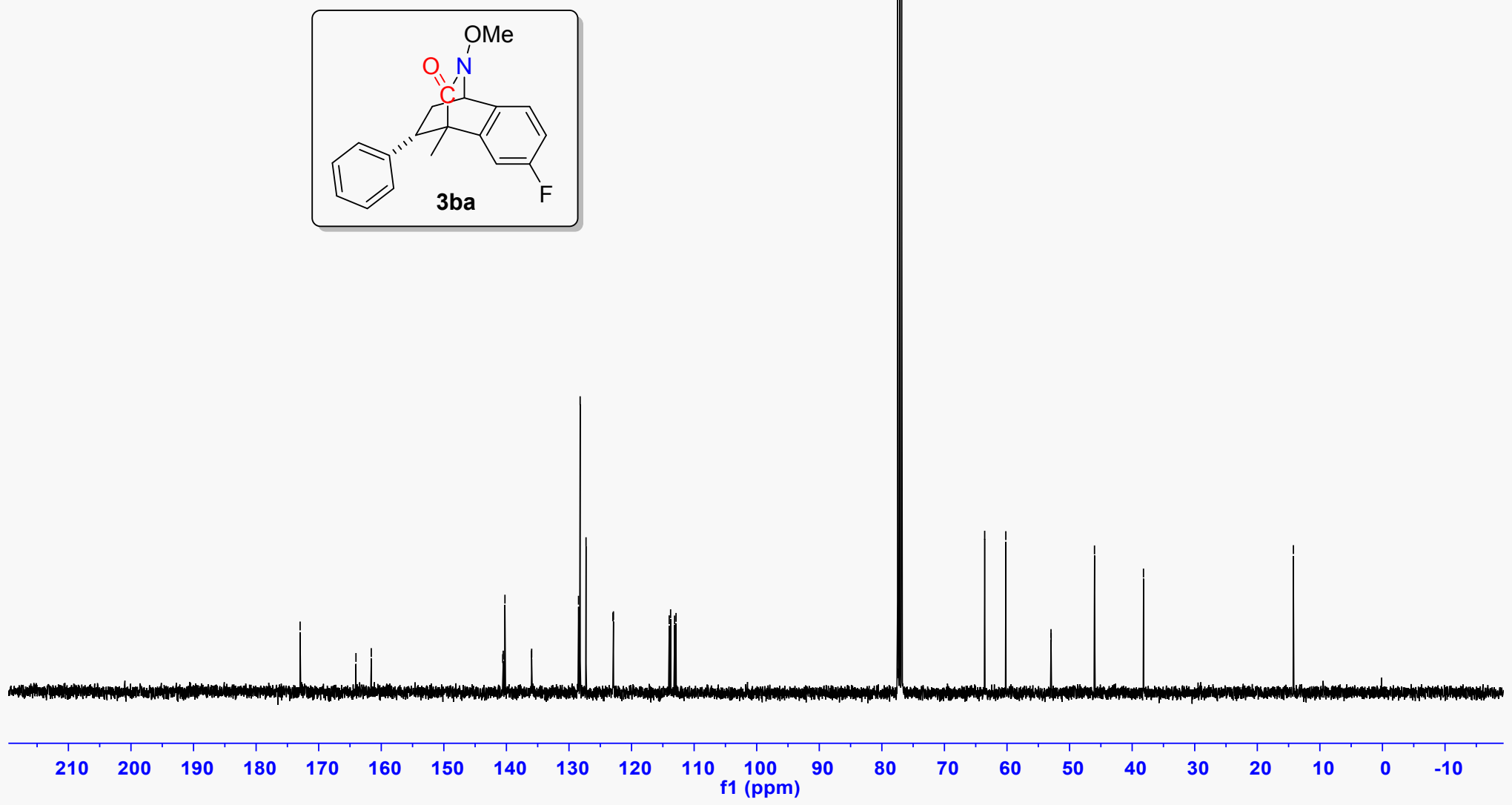
3ba-F (376 MHz, CDCl3)

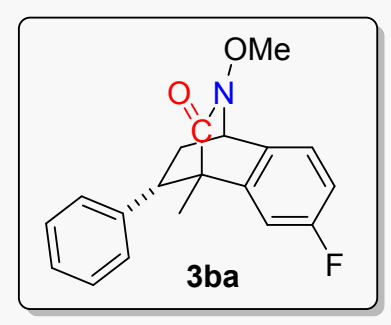


$3 \mathrm{ca}-\mathrm{H}(400 \mathrm{MHz}, \mathrm{CDCl} 3)$

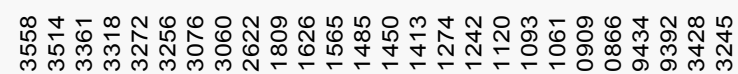

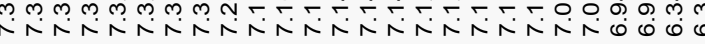

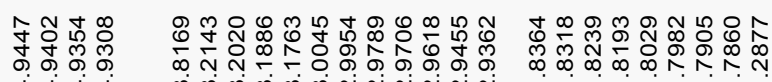

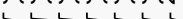

(45T5
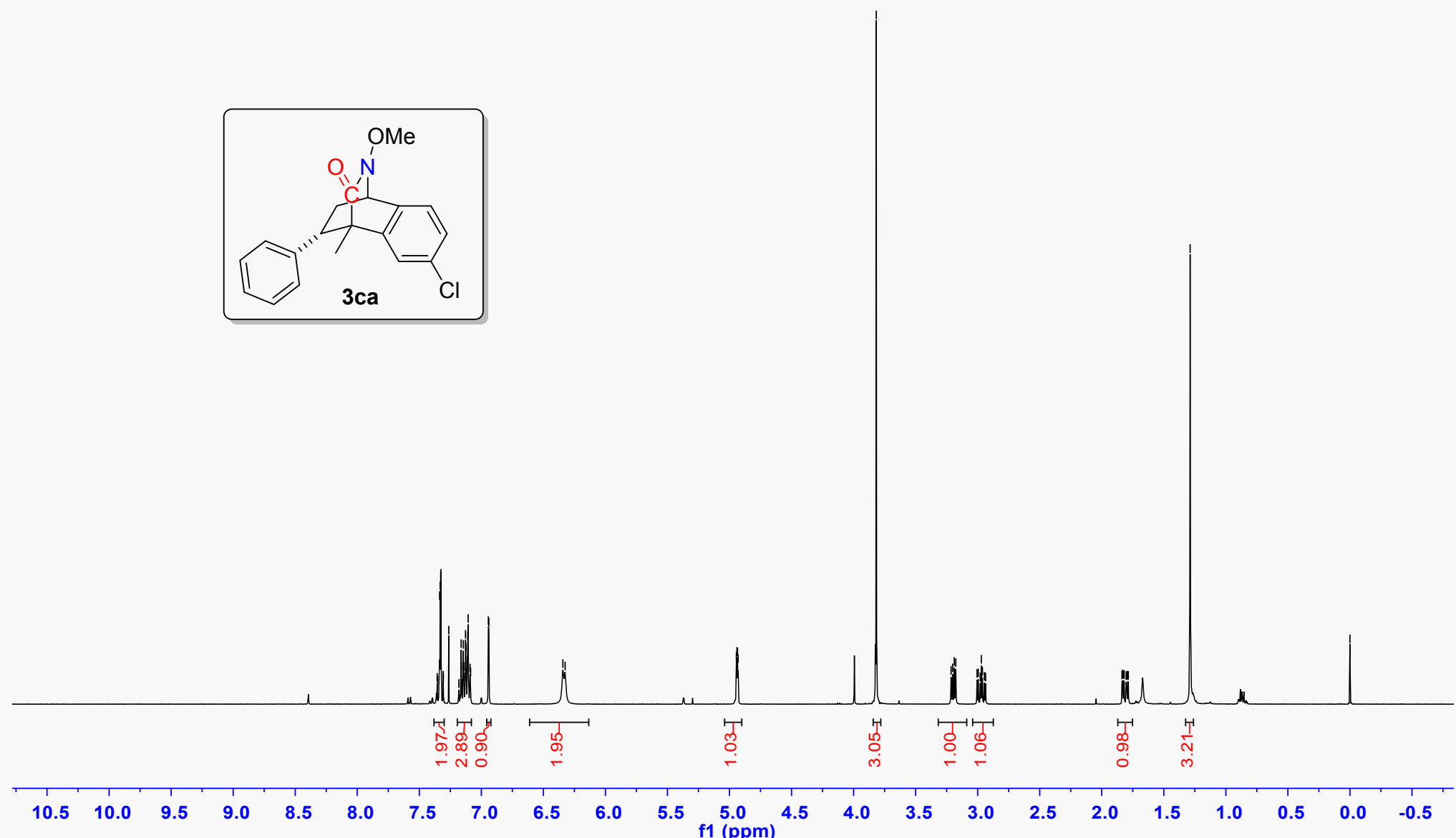

$\begin{array}{lll}10.5 & 10.0 & 9.5\end{array}$
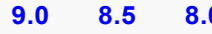

$7.5 \quad 7.0$

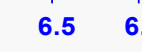

$5.5 \underset{f 1}{5.0}(\mathrm{ppm})$

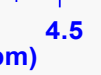



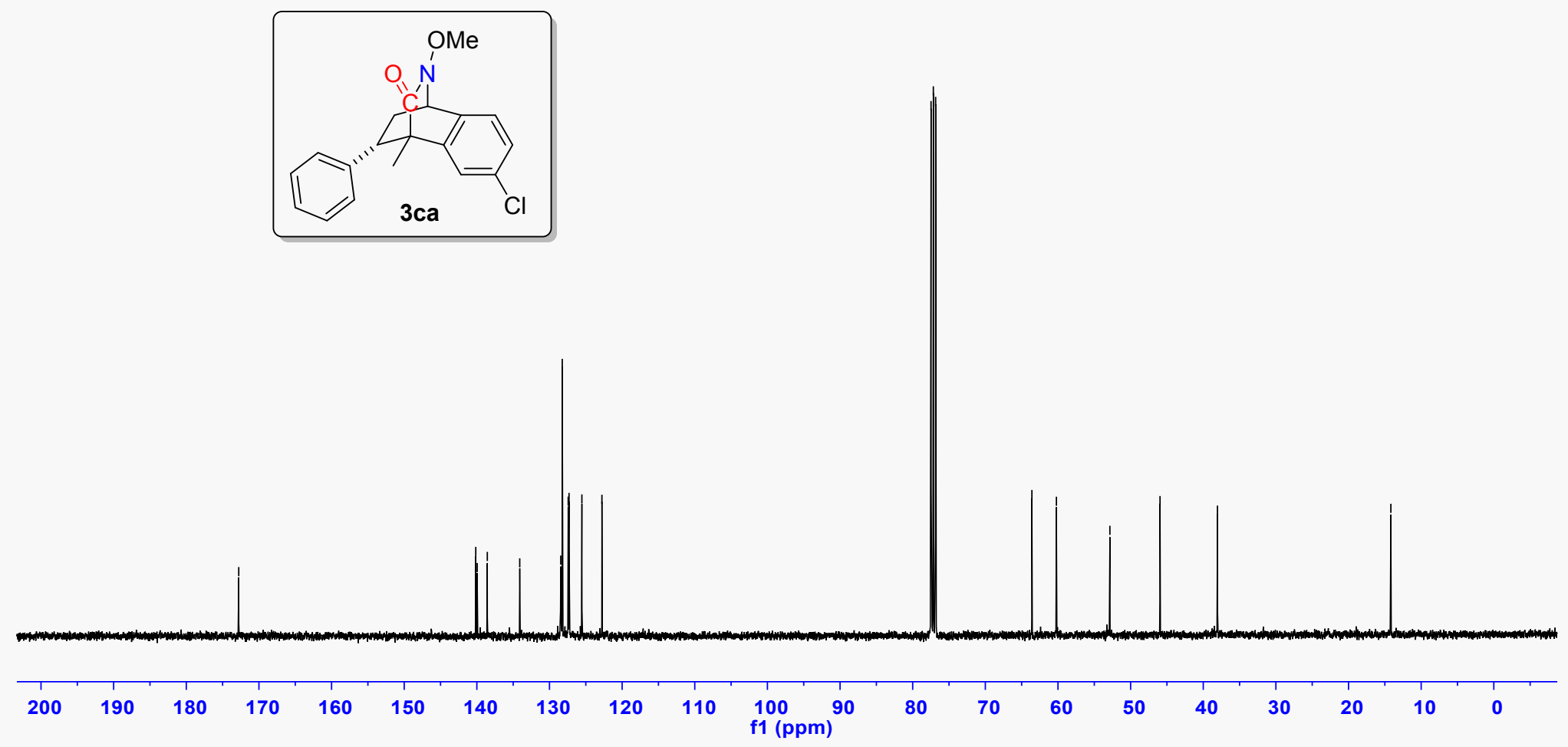


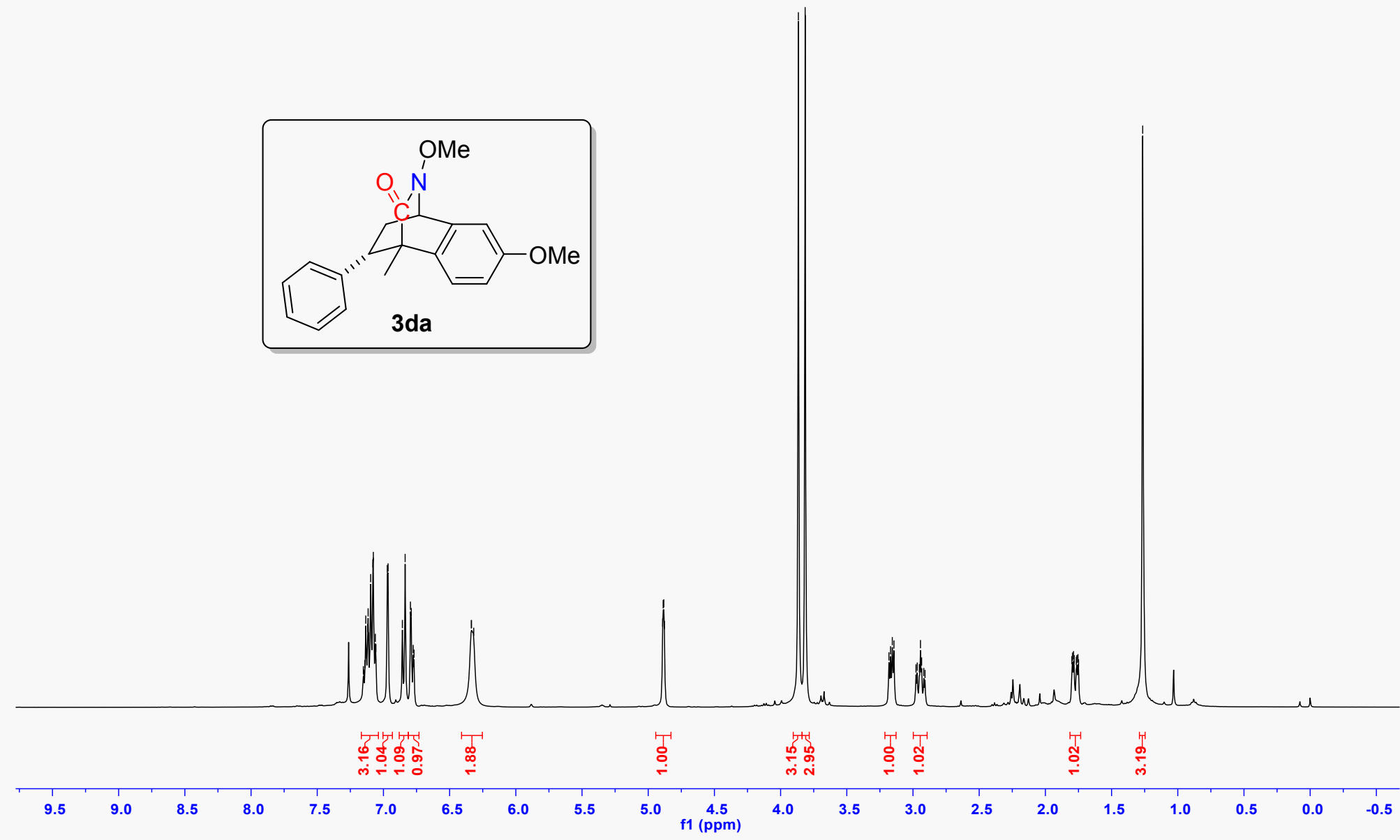



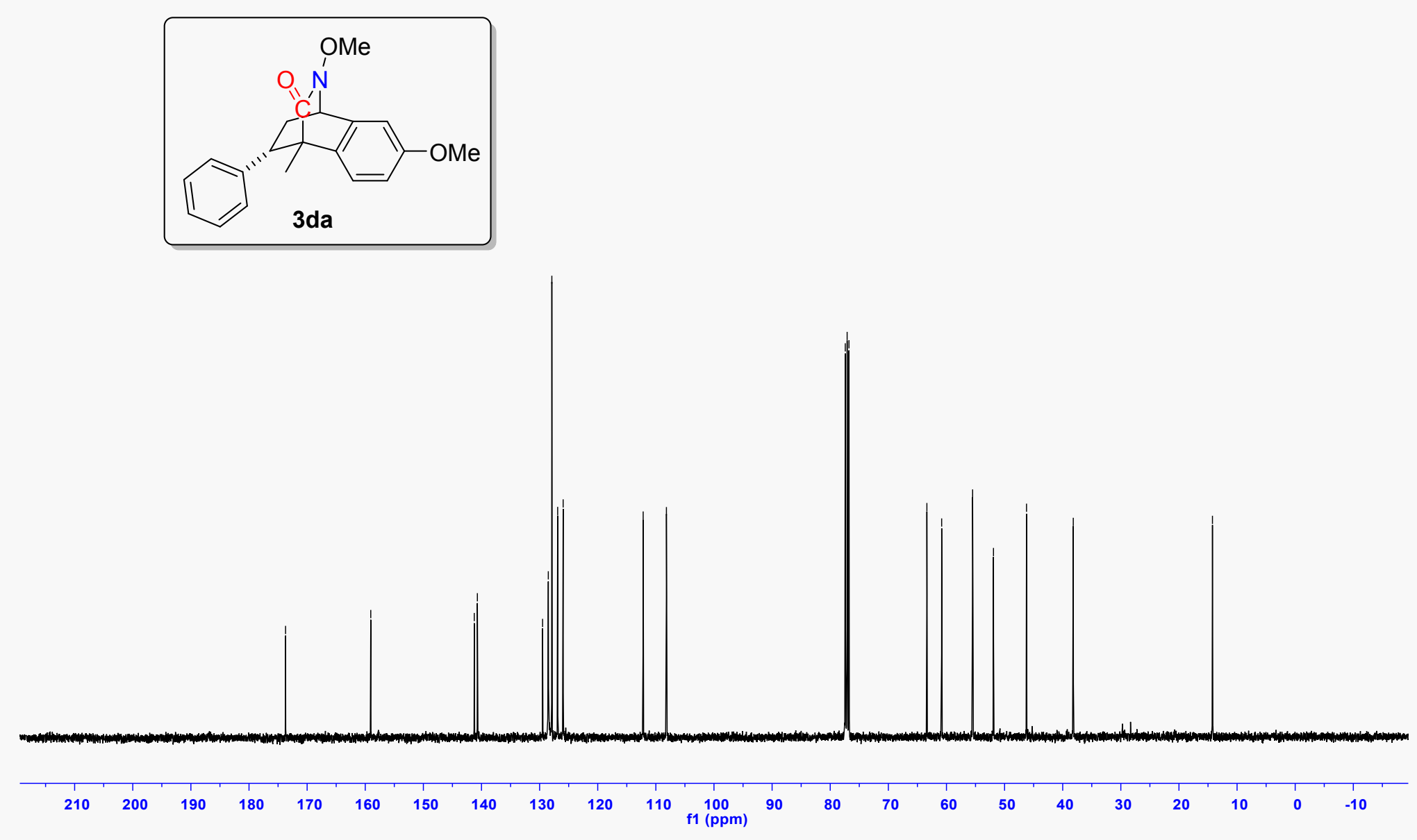
3ea-H (400 MHz, CDCl3)

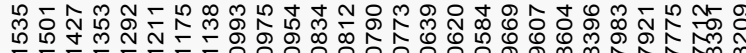

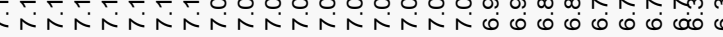

(1)

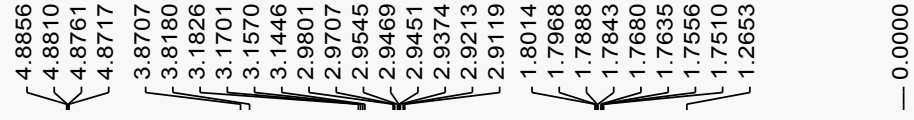

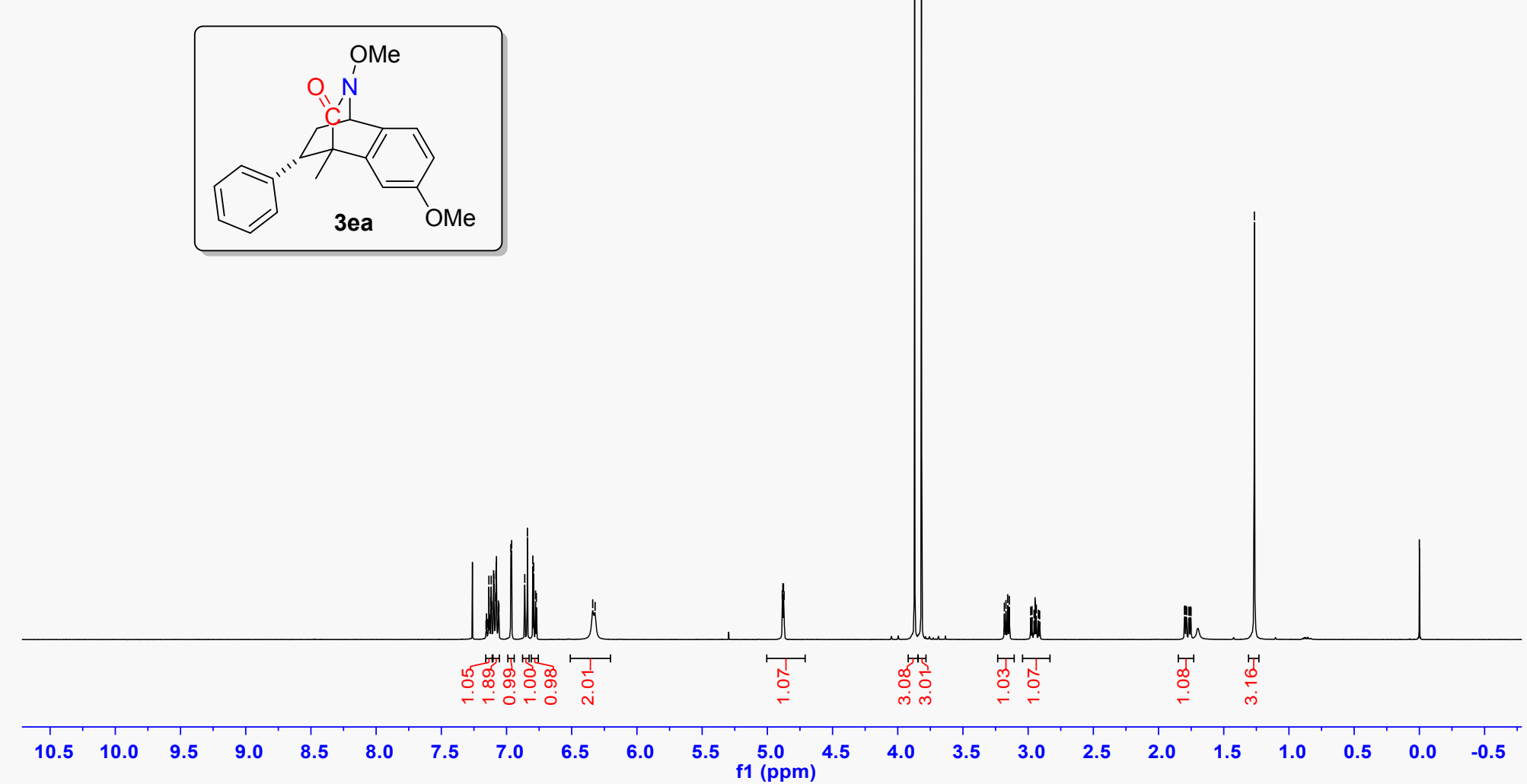


3ea-C(100 MHz, CDCl3)

\begin{tabular}{|c|c|c|c|c|c|c|}
\hline 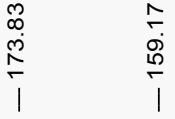 & 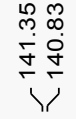 & 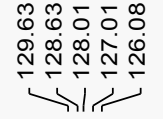 & 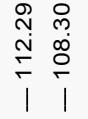 & 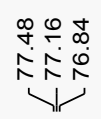 & 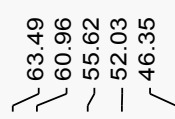 & $\begin{array}{c}\text { p } \\
\infty \\
\infty \\
m \\
\text { । }\end{array}$ \\
\hline
\end{tabular}
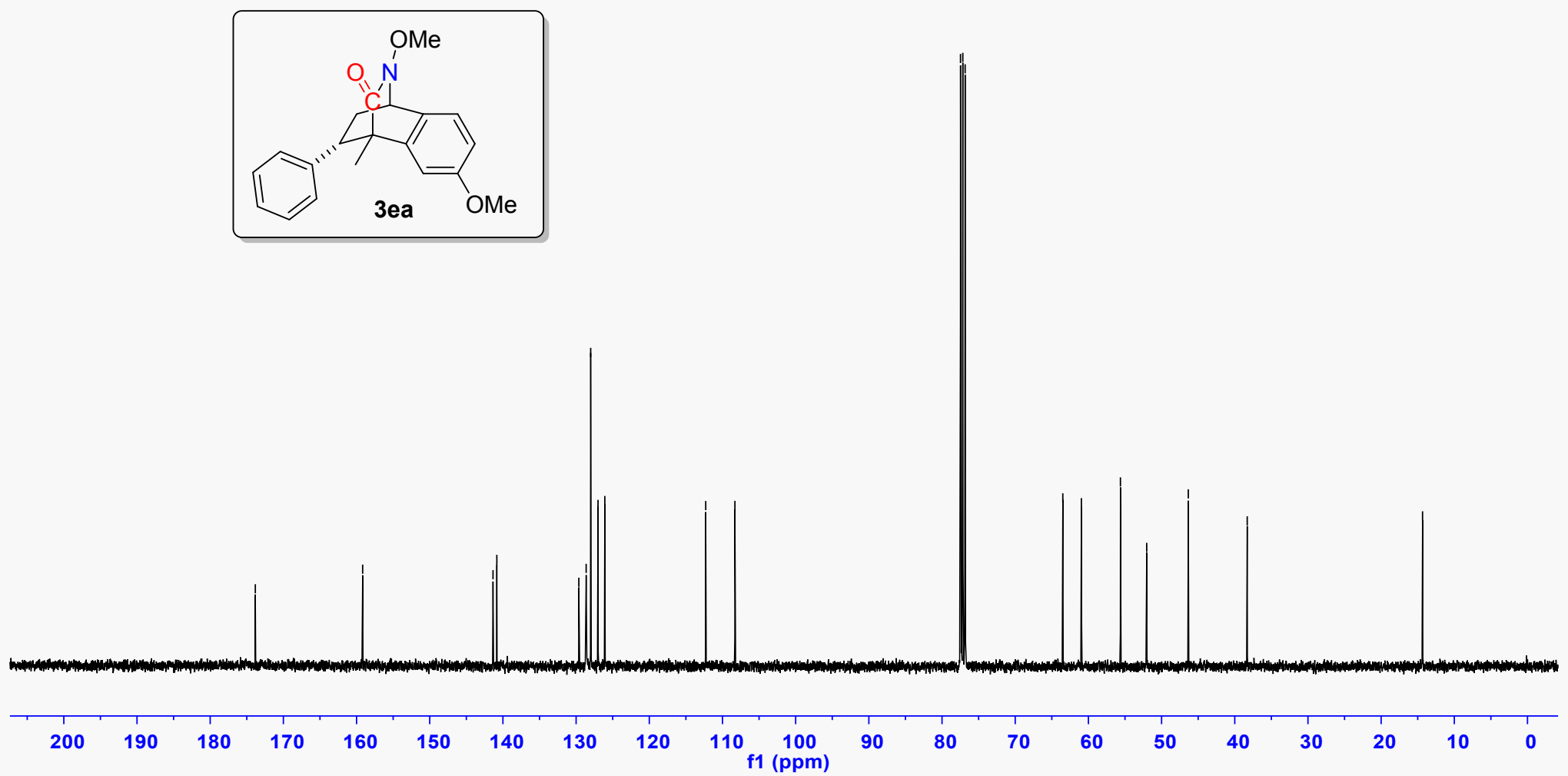


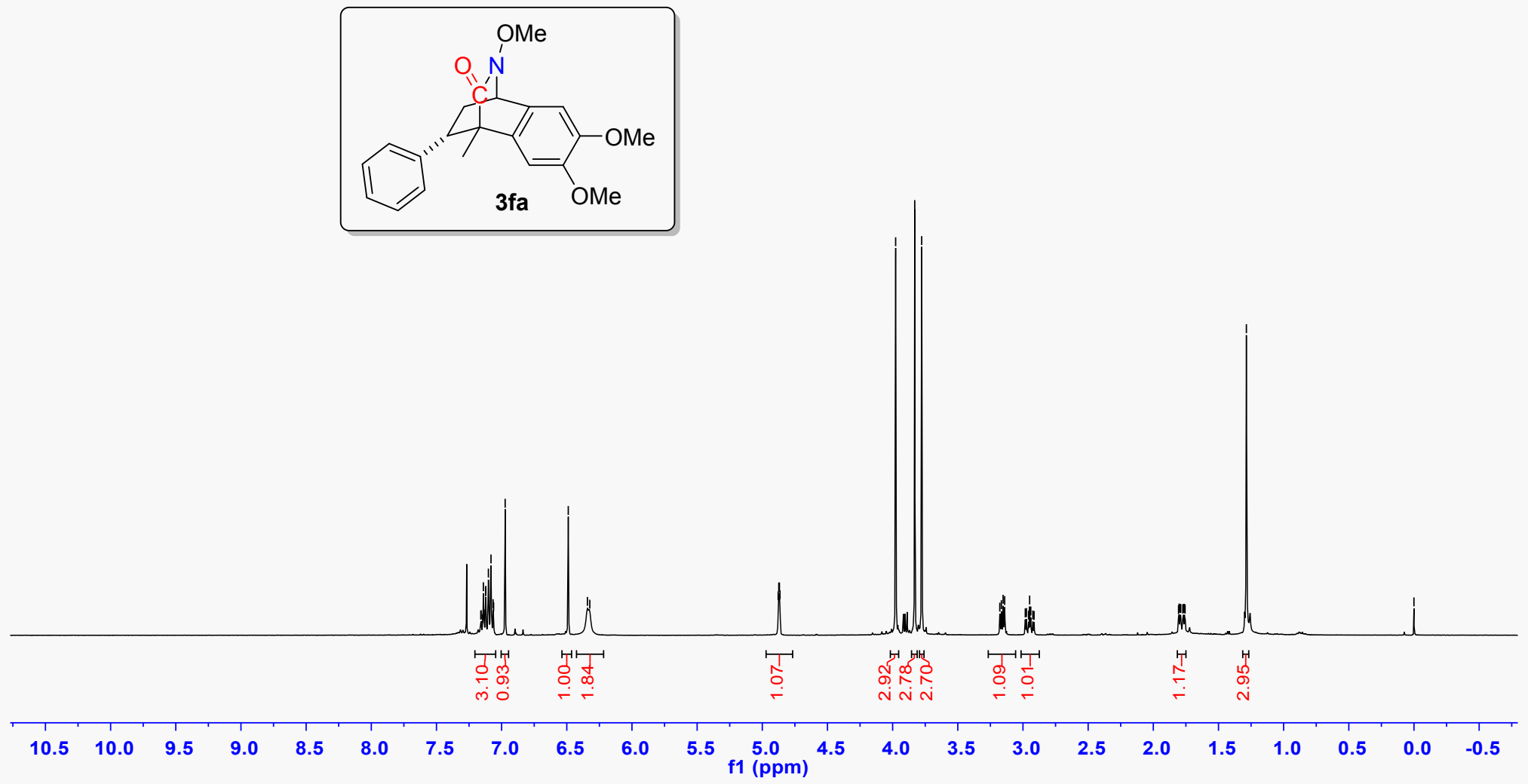


3fa-C (100 MHz, CDCl3)

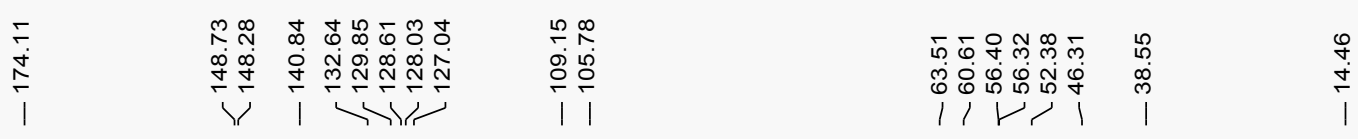

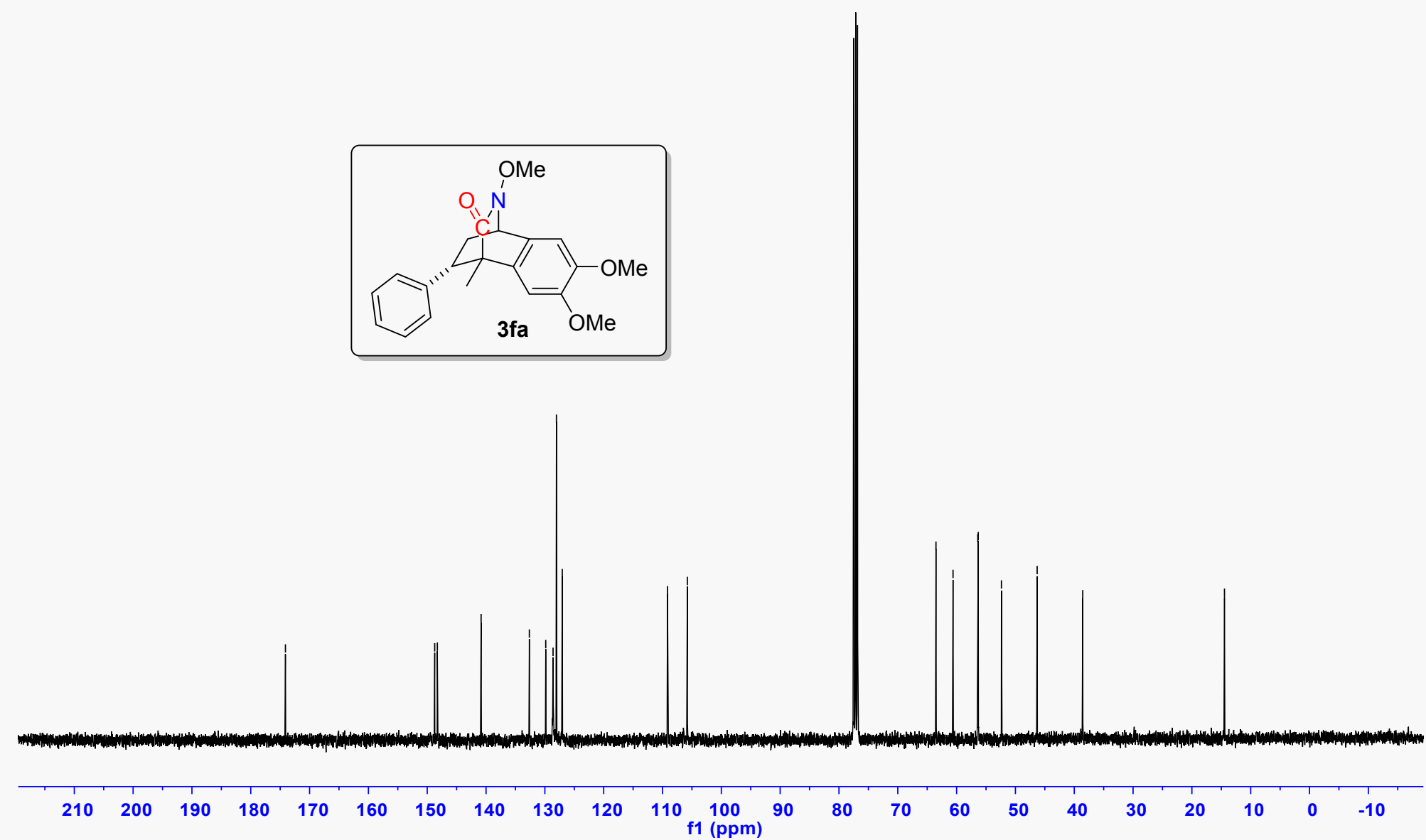


3ga-H (400 MHz, CDC13)

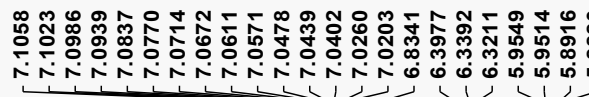

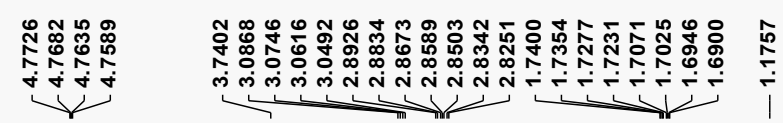

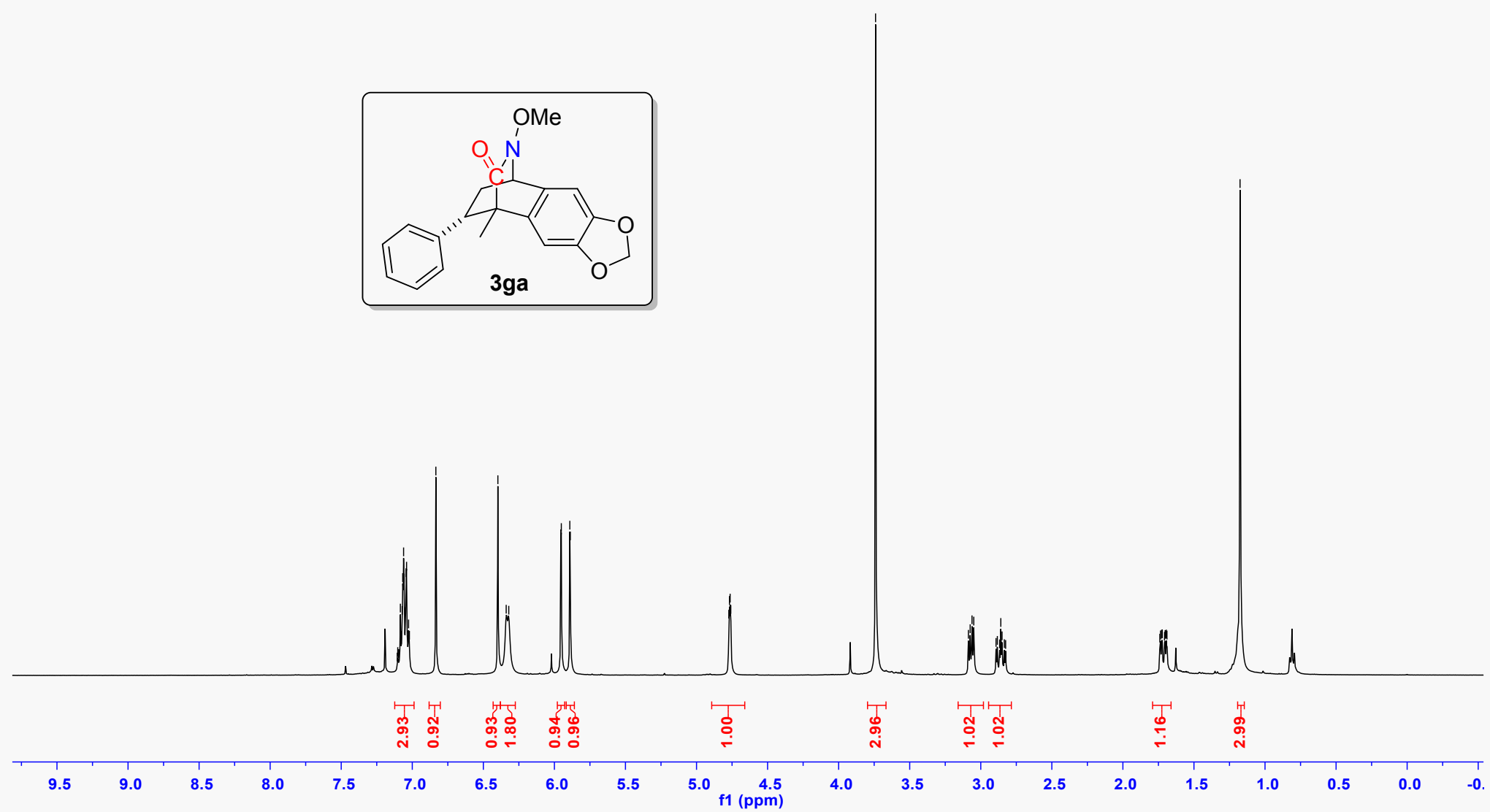




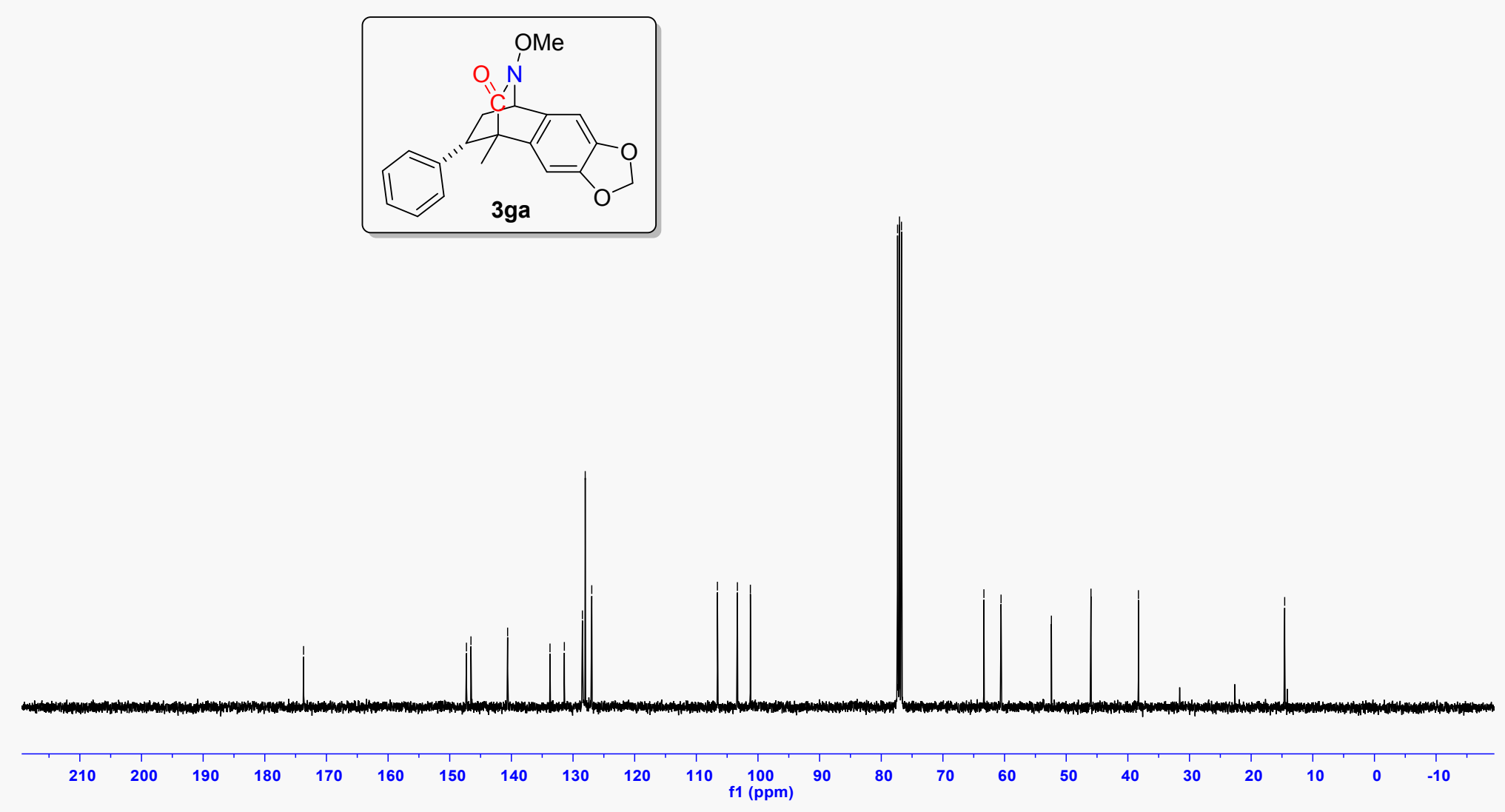




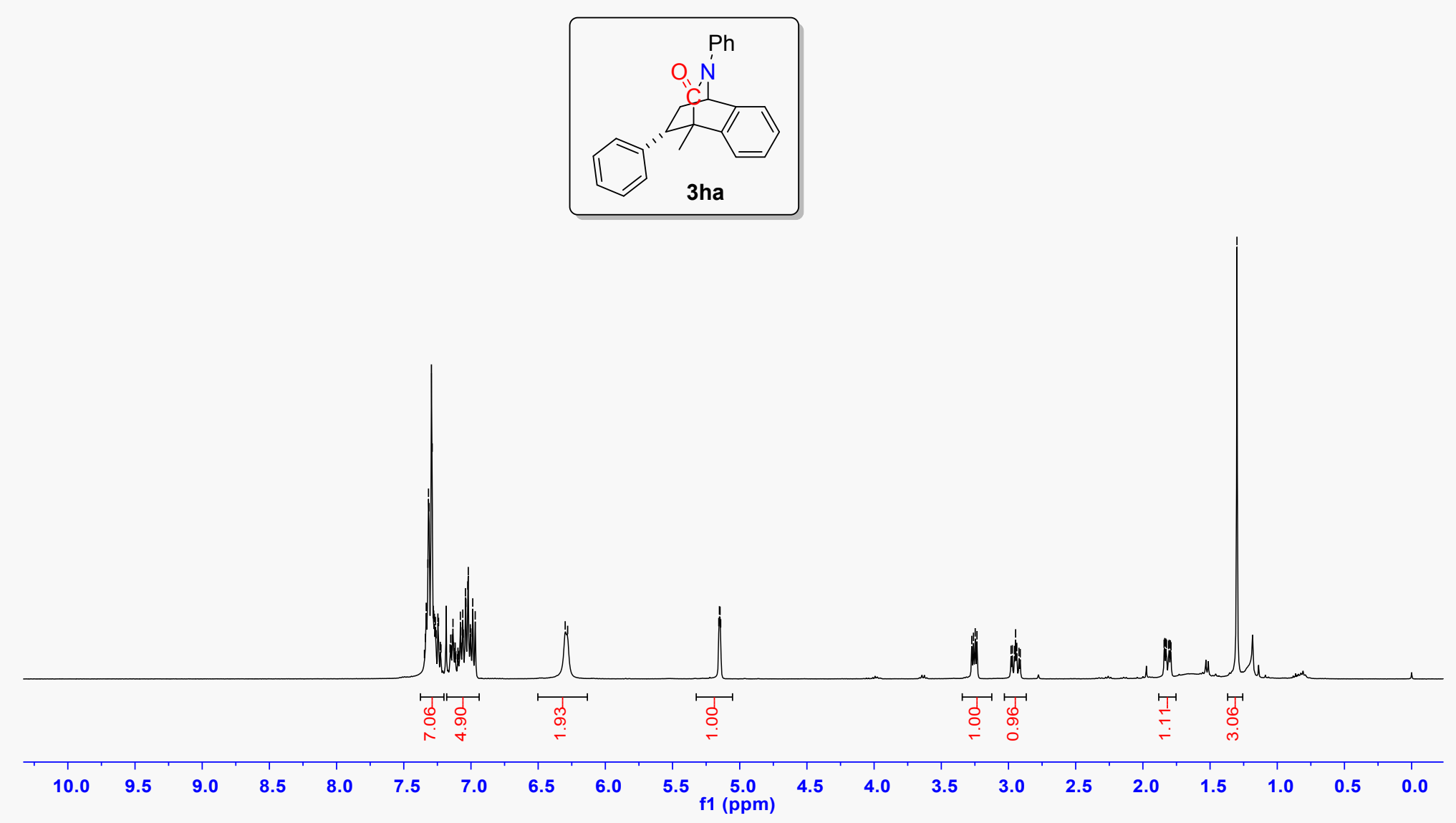




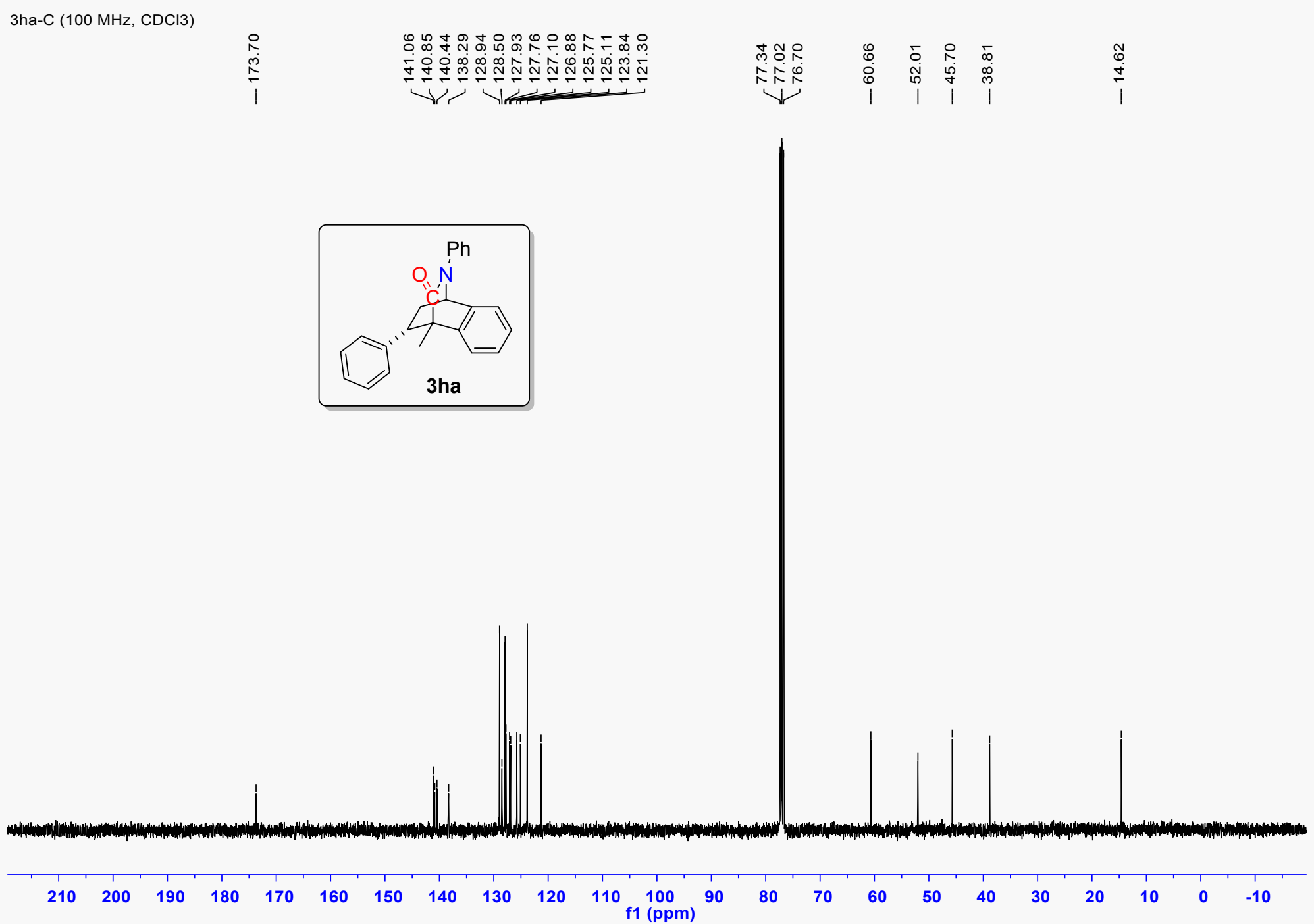


3ab-H (400 MHz, CDCl3)

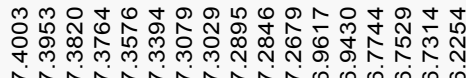

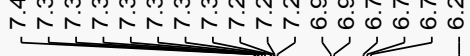

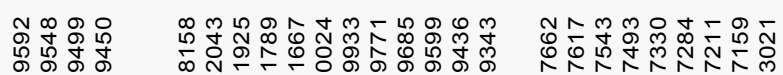

$\checkmark+4$

$\sqrt[n]{.292}$

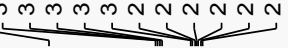

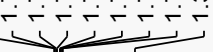

0
$\vdots$
$\vdots$
$\vdots$
1

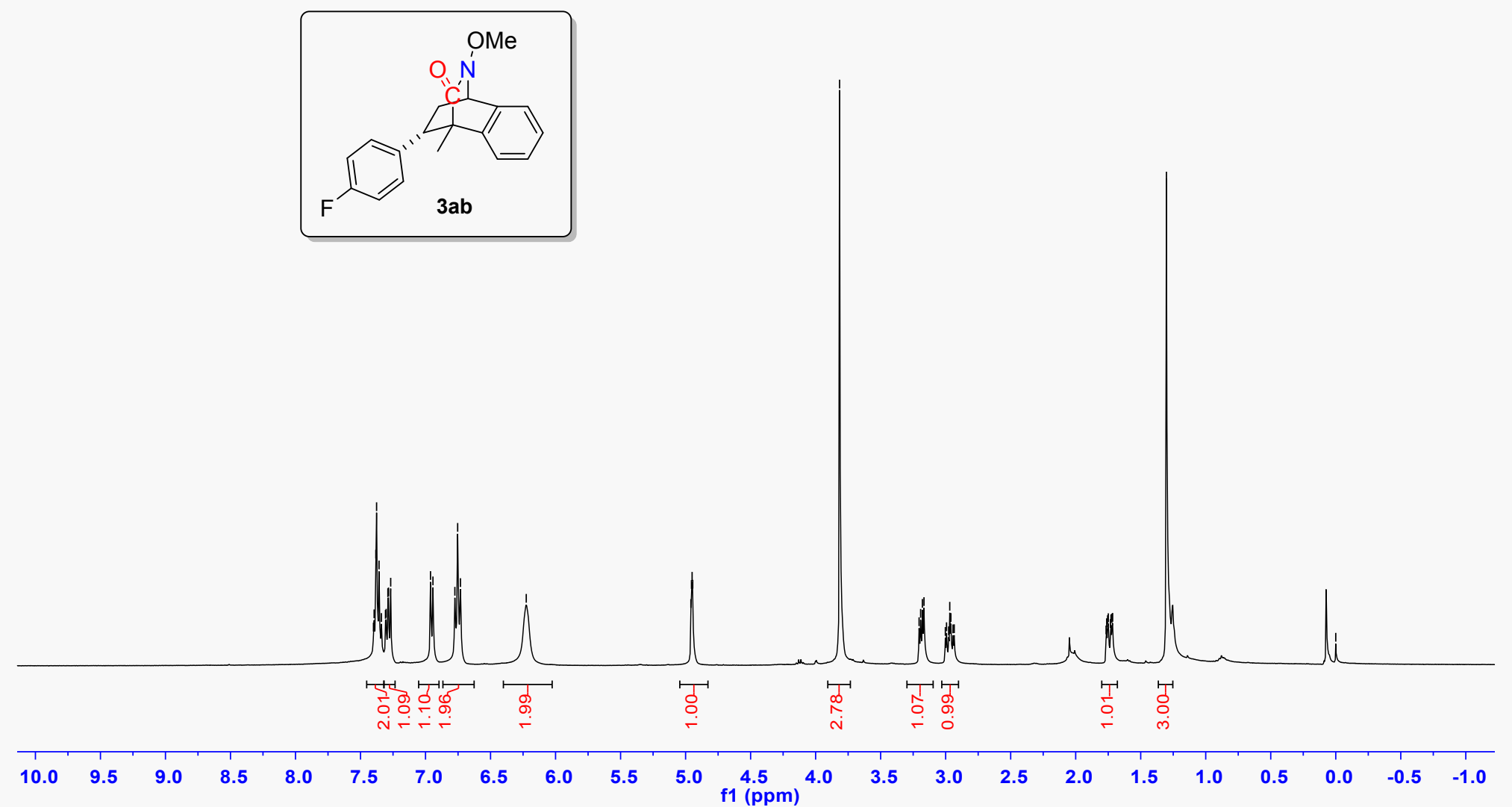


3ab-C (100 MHz, CDCl3)
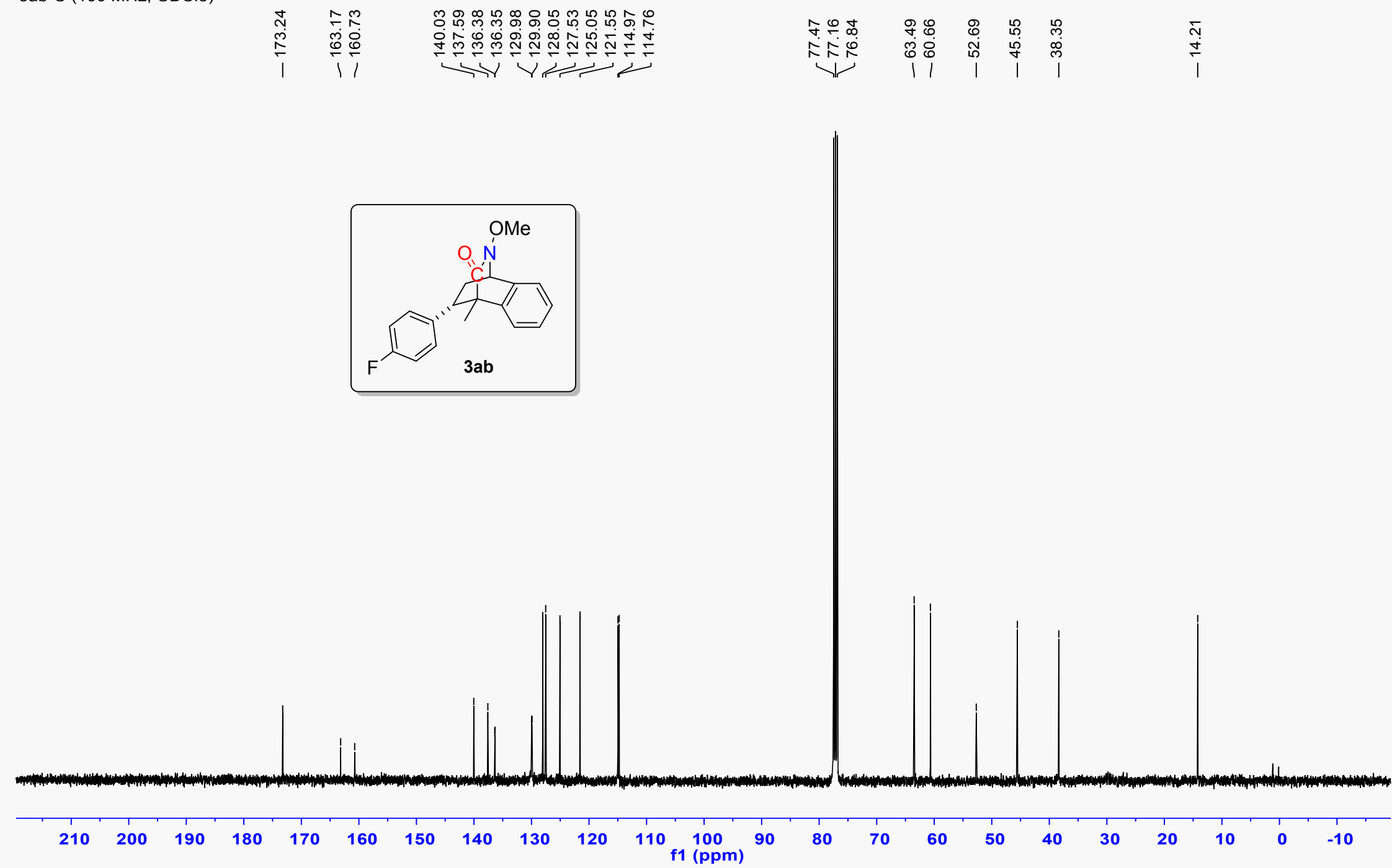
3ab-F (376 MHz, CDC13)
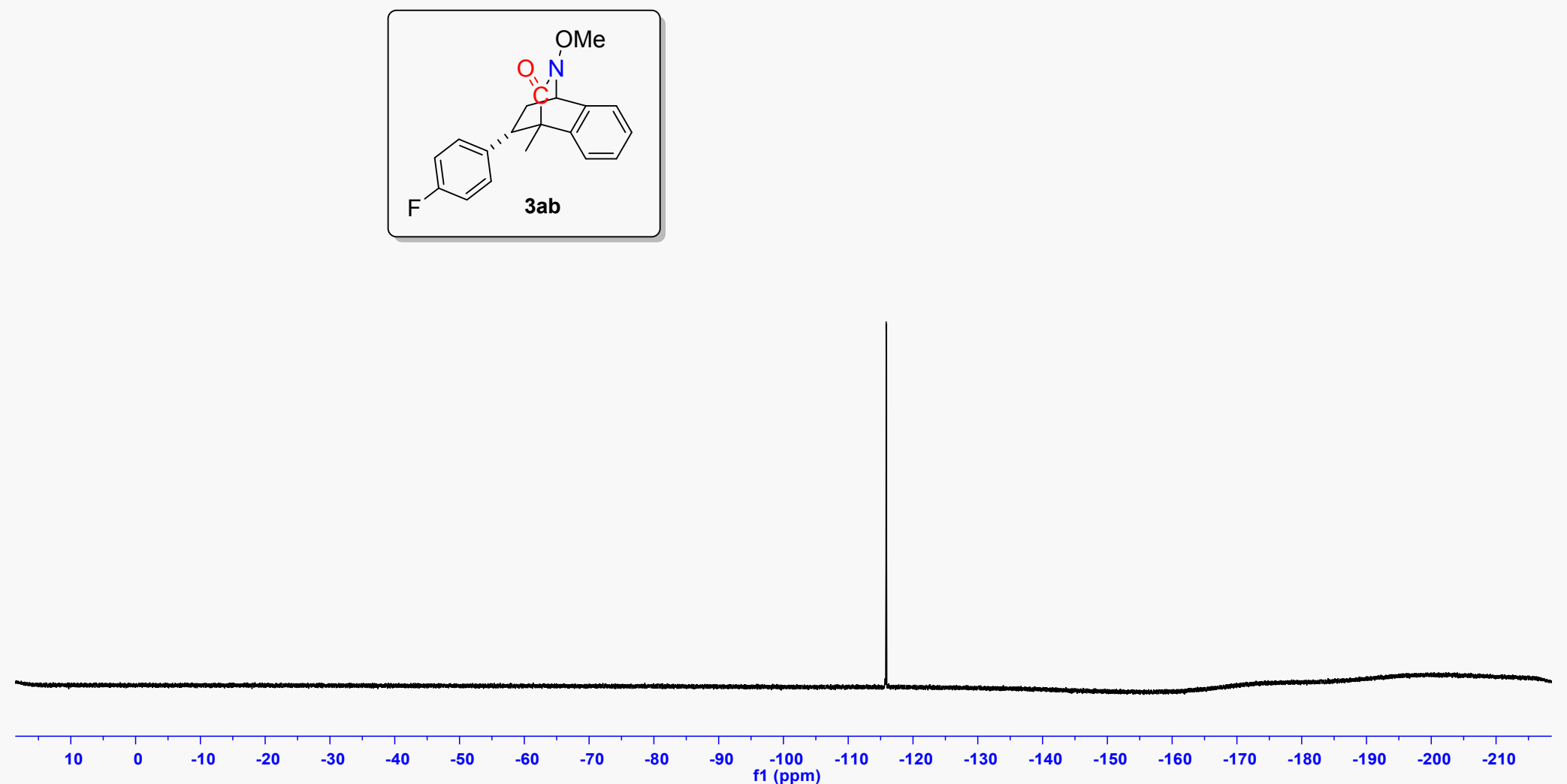


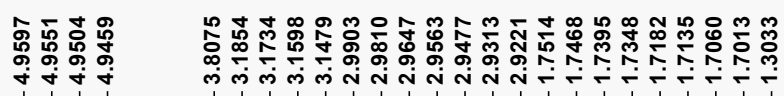
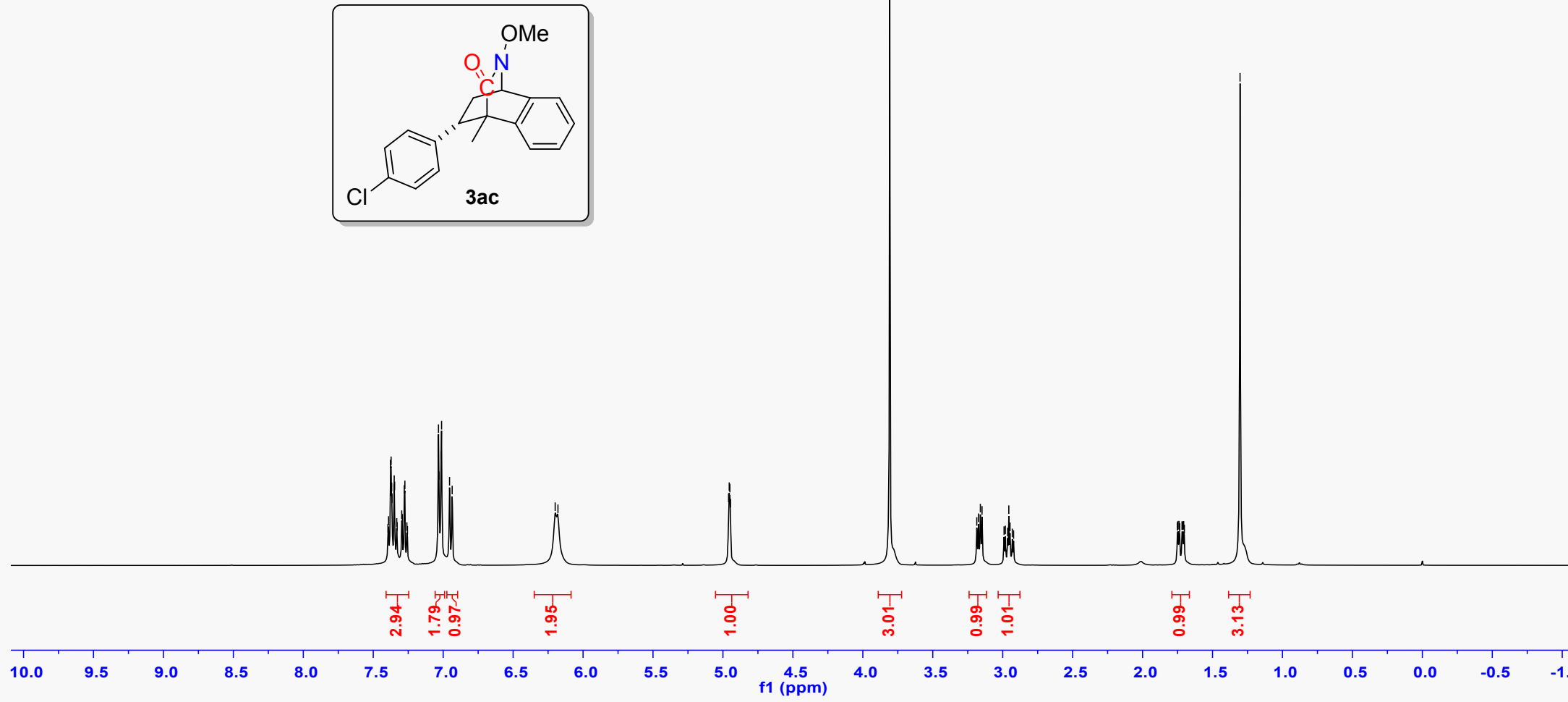
3ac-C $(100 \mathrm{MHz}, \quad \mathrm{CDC} 13)$

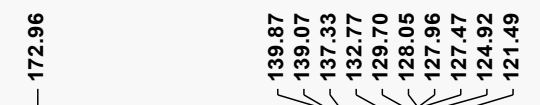

许
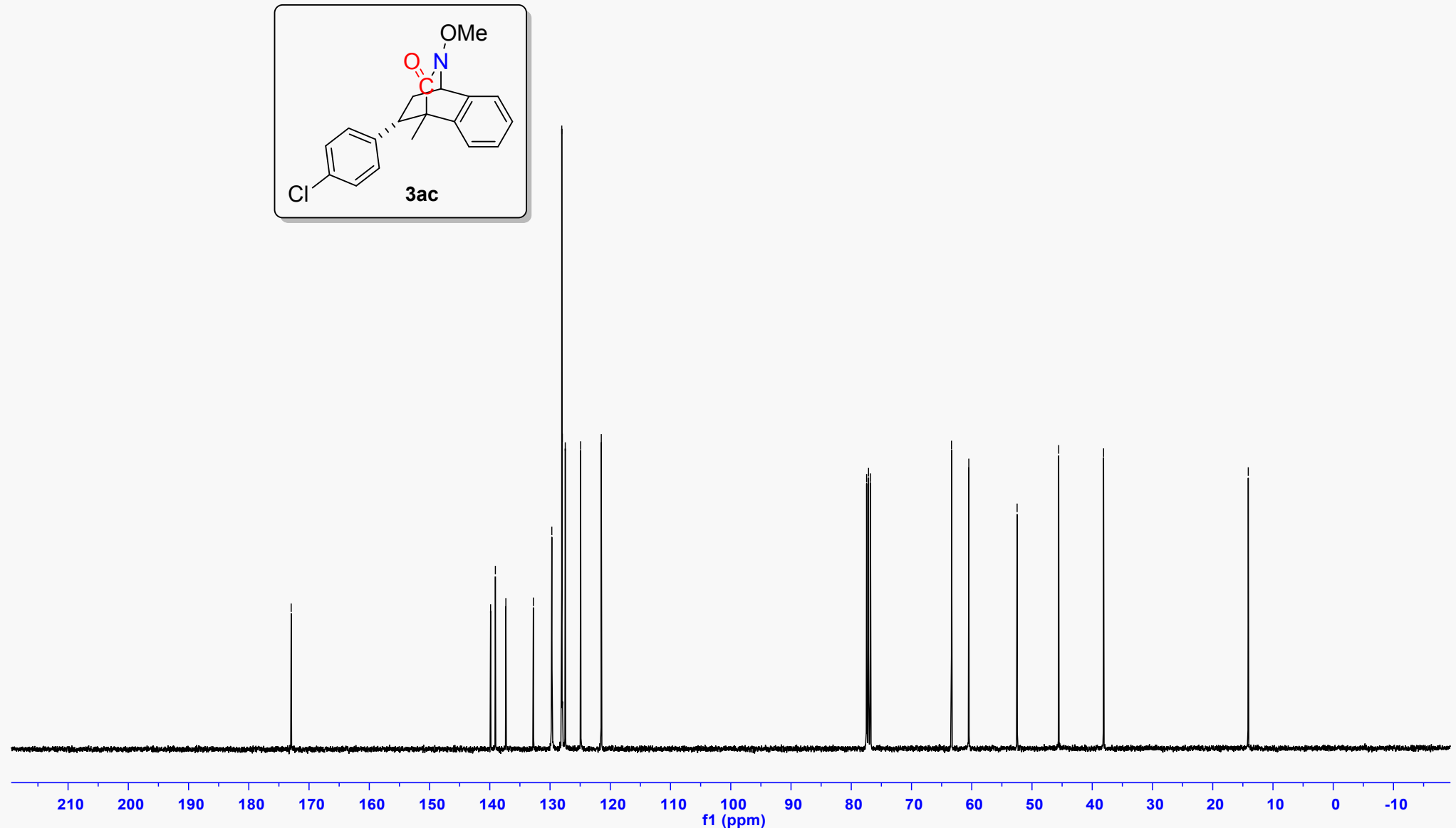

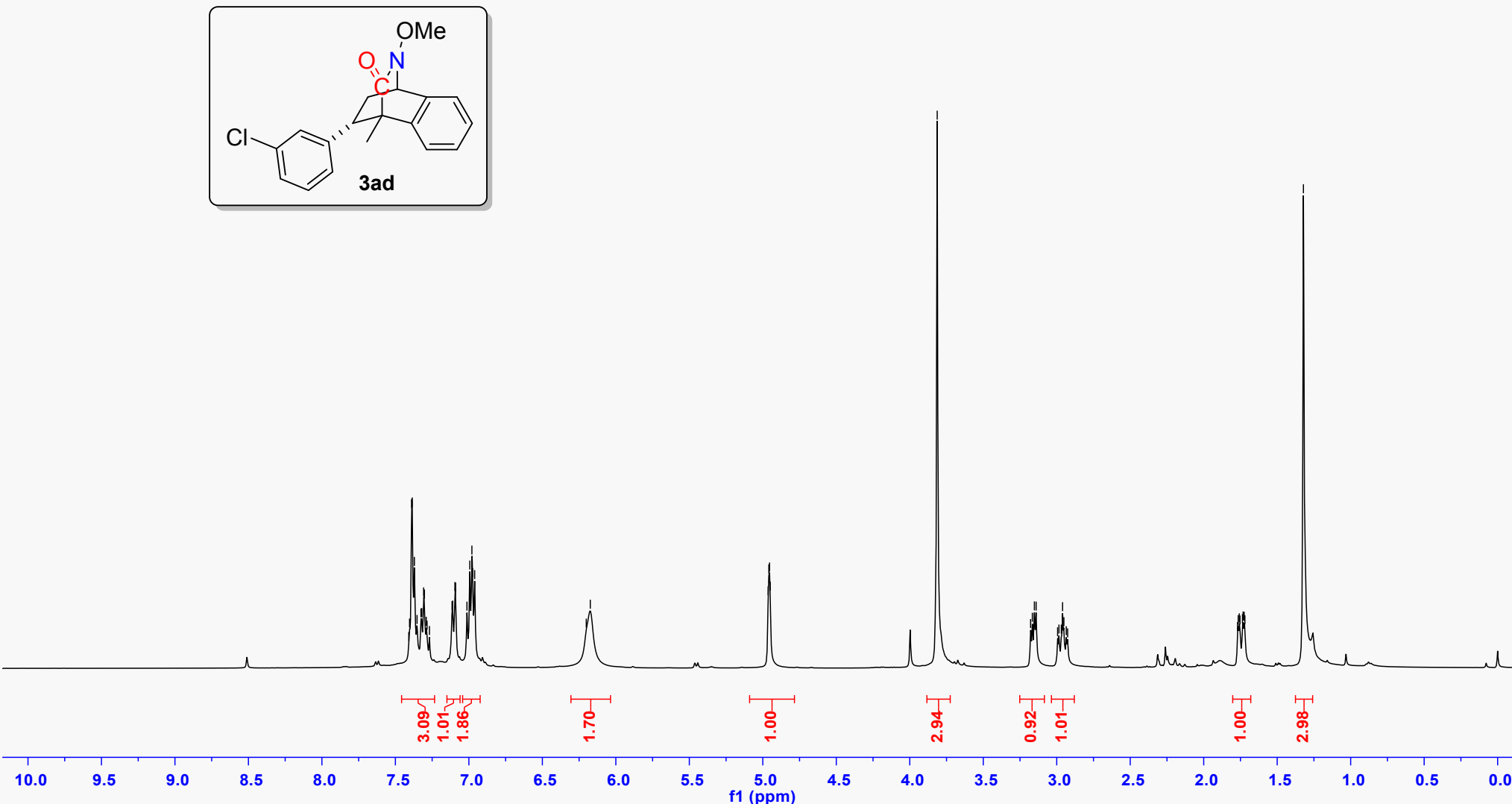
3ad-C (100 MHz, CDC13)

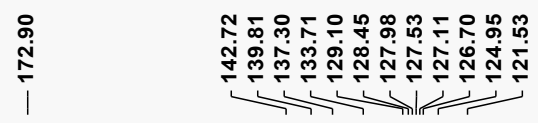

至

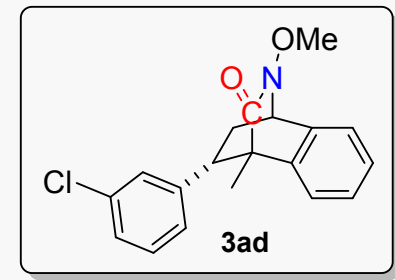

OM

ad

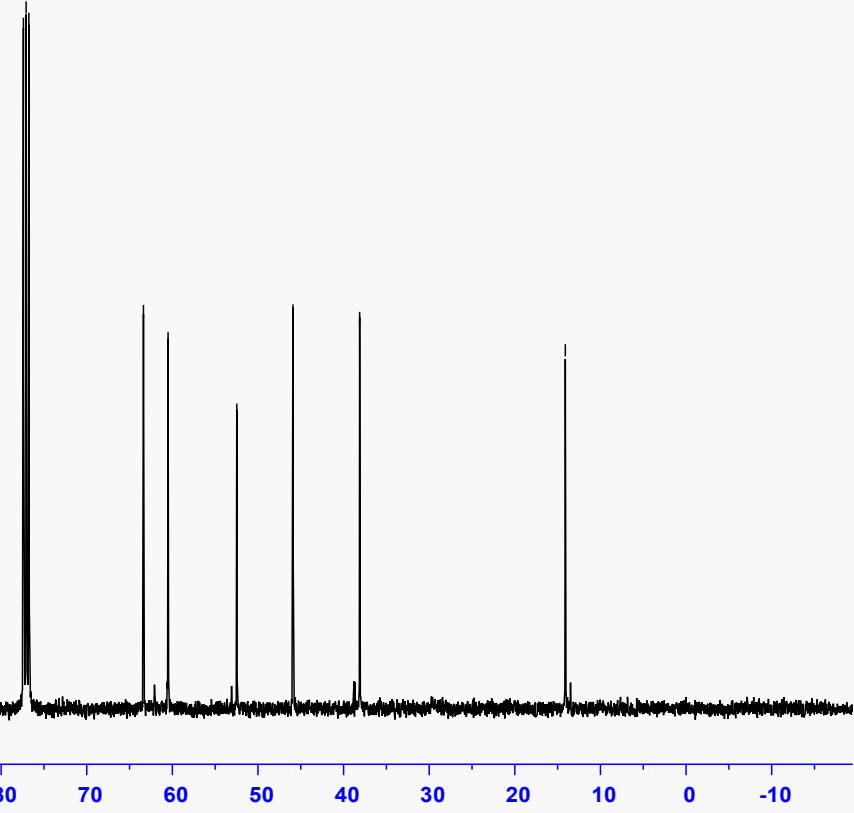




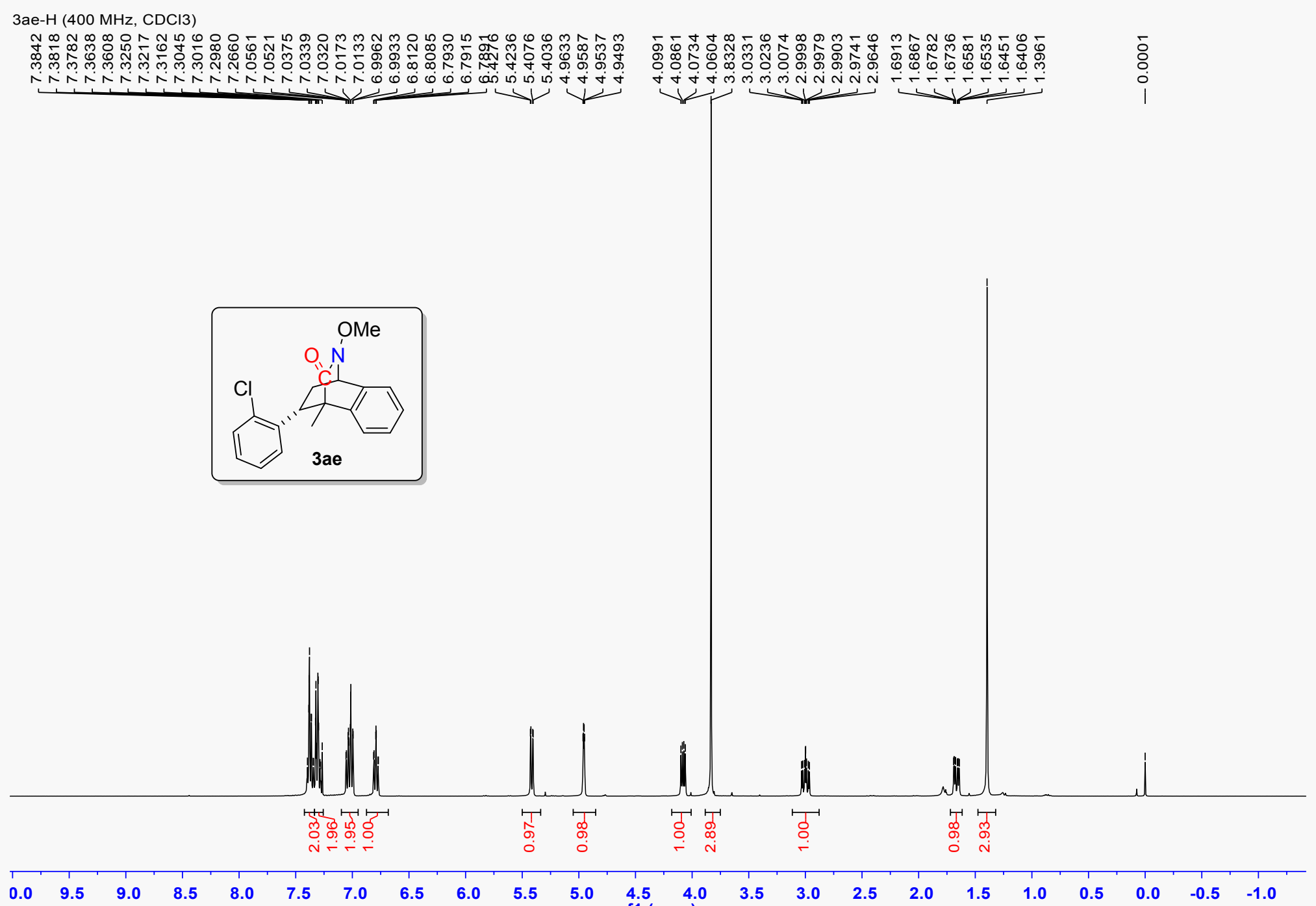

$\begin{array}{lllllllllllll}0.0 & 9.5 & 9.0 & 8.5 & 8.0 & 7.5 & 7.0 & 6.5 & 6.0 & 5.5 & 5.0 & \begin{array}{l}4.5 \\ \mathrm{f} 1(\mathrm{ppm})\end{array}\end{array}$ 
3ae-C (100 MHz, CDCl3)

\begin{tabular}{|c|c|c|c|c|c|c|}
\hline 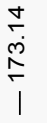 & 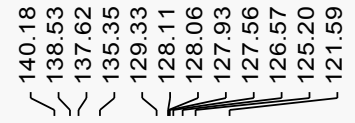 & 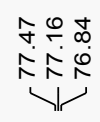 & 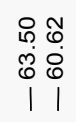 & 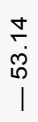 & 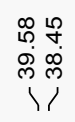 & 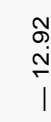 \\
\hline
\end{tabular}
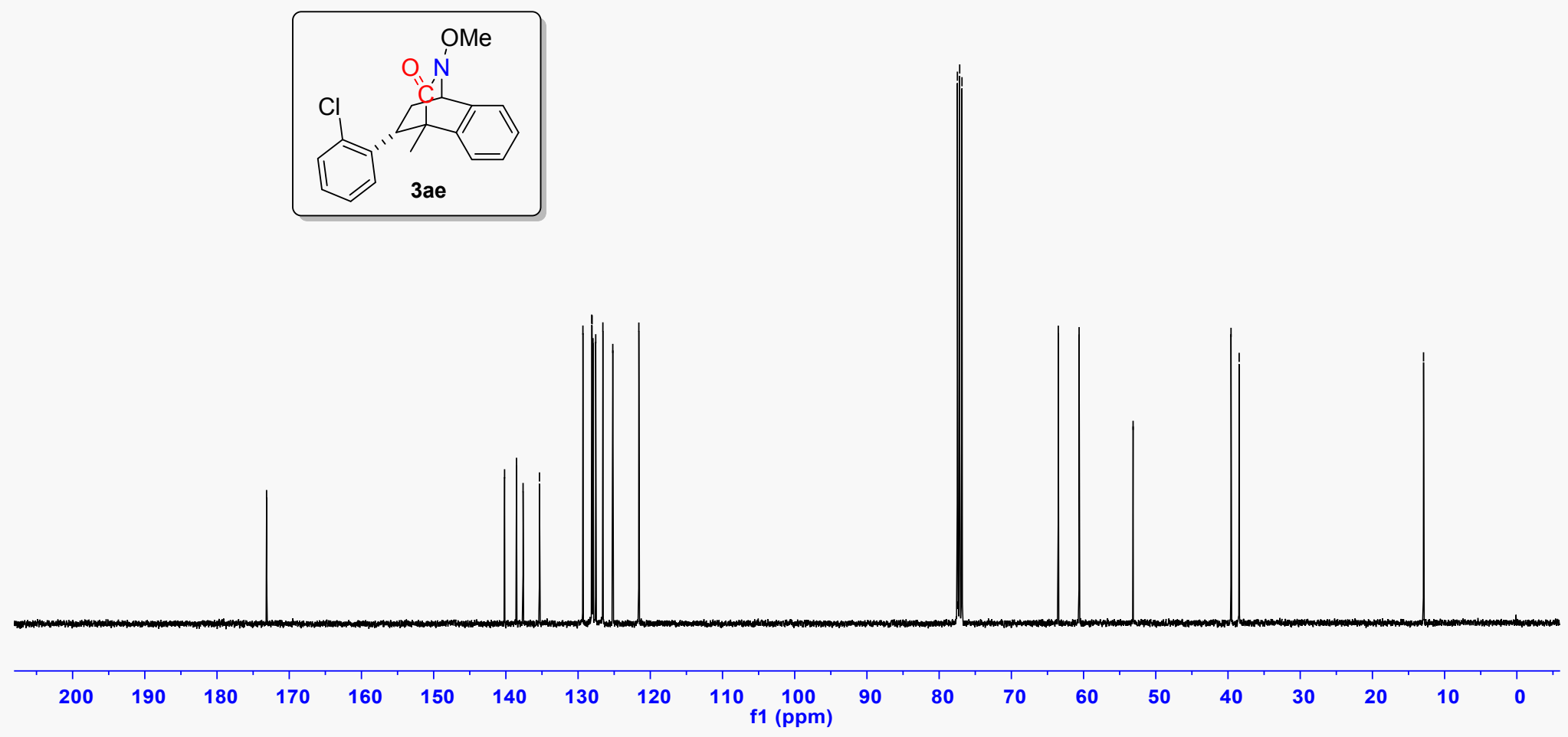


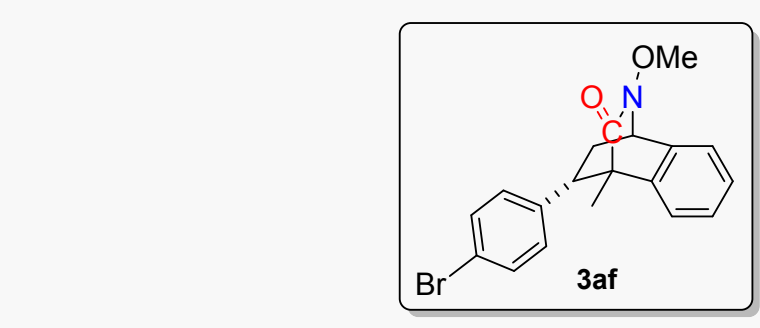

$\mathrm{OM}$
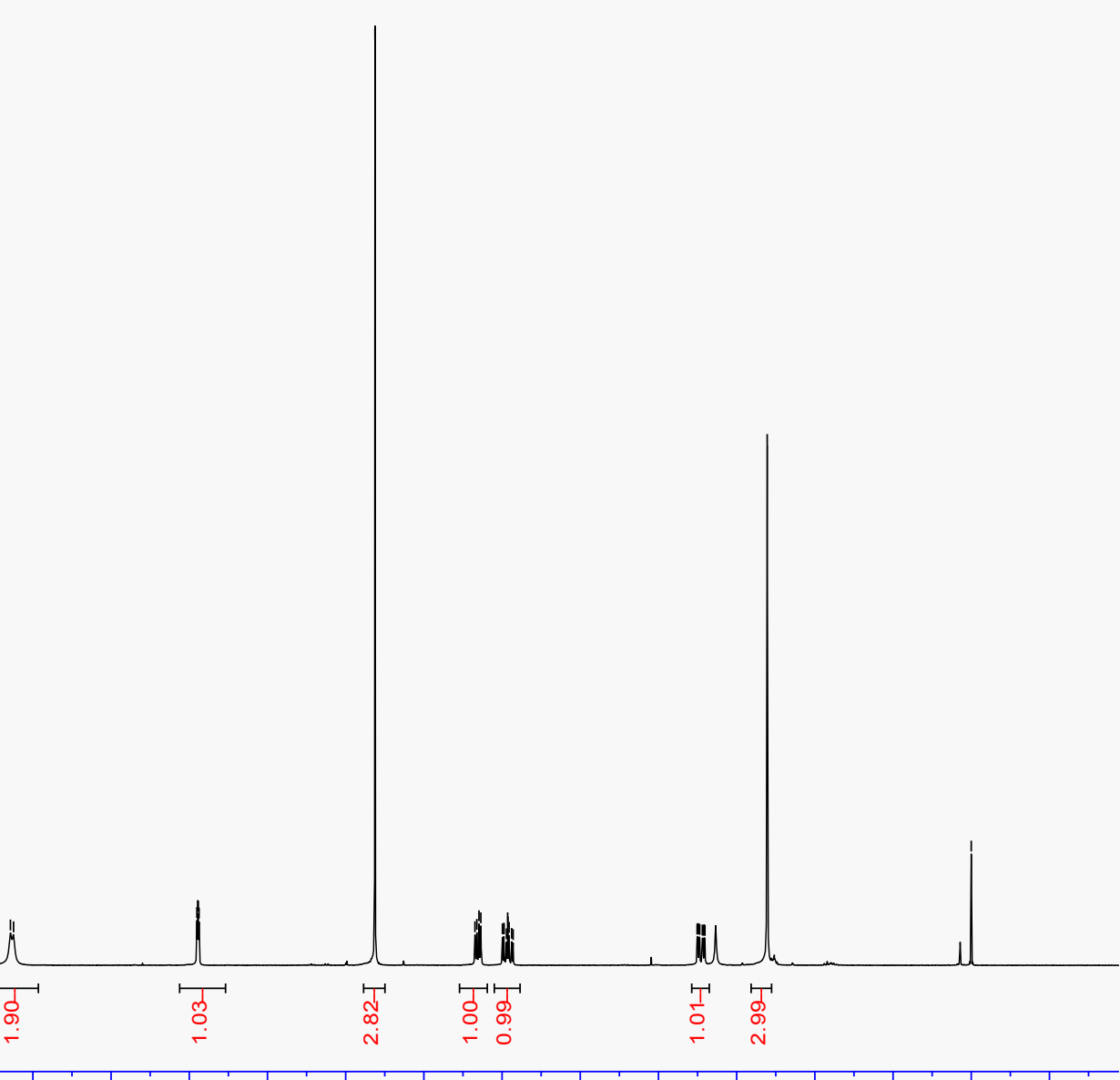

$\begin{array}{llllllllllll}10.5 & 10.0 & 9.5 & 9.0 & 8.5 & 8.0 & 7.5 & 7.0 & 6.5 & 6.0 & 5.5 & \begin{array}{c}5.0 \\ \mathrm{f} 1(\mathrm{ppm})\end{array} \\ & & & & & & & & & & \end{array}$ 
3af-C (100 MHz, $\mathrm{CDCl} 3)$
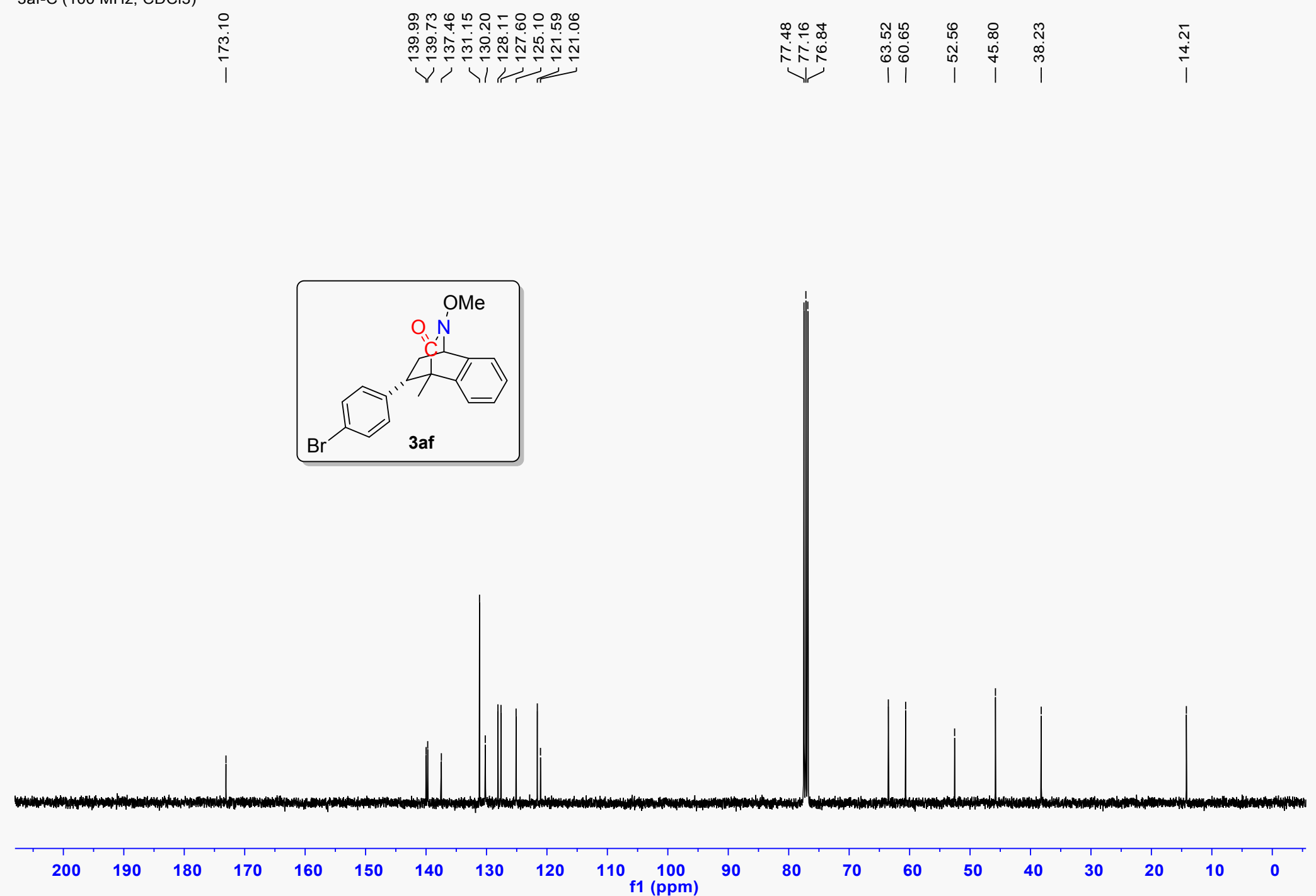
3ag- $\mathrm{H}(400 \mathrm{MHz}, \mathrm{CDCl} 3)$

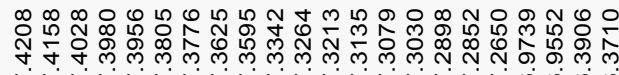

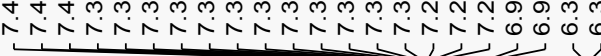
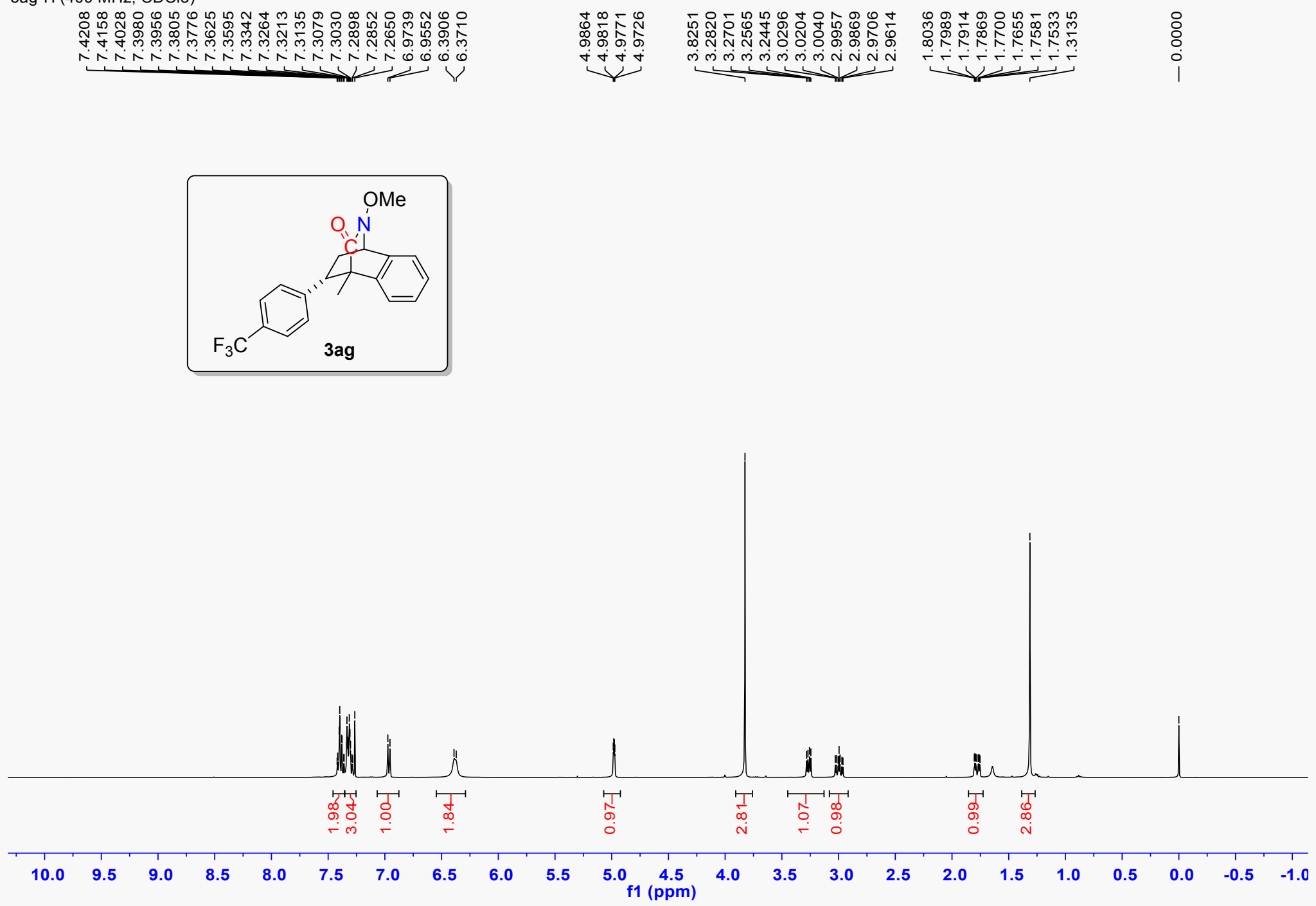


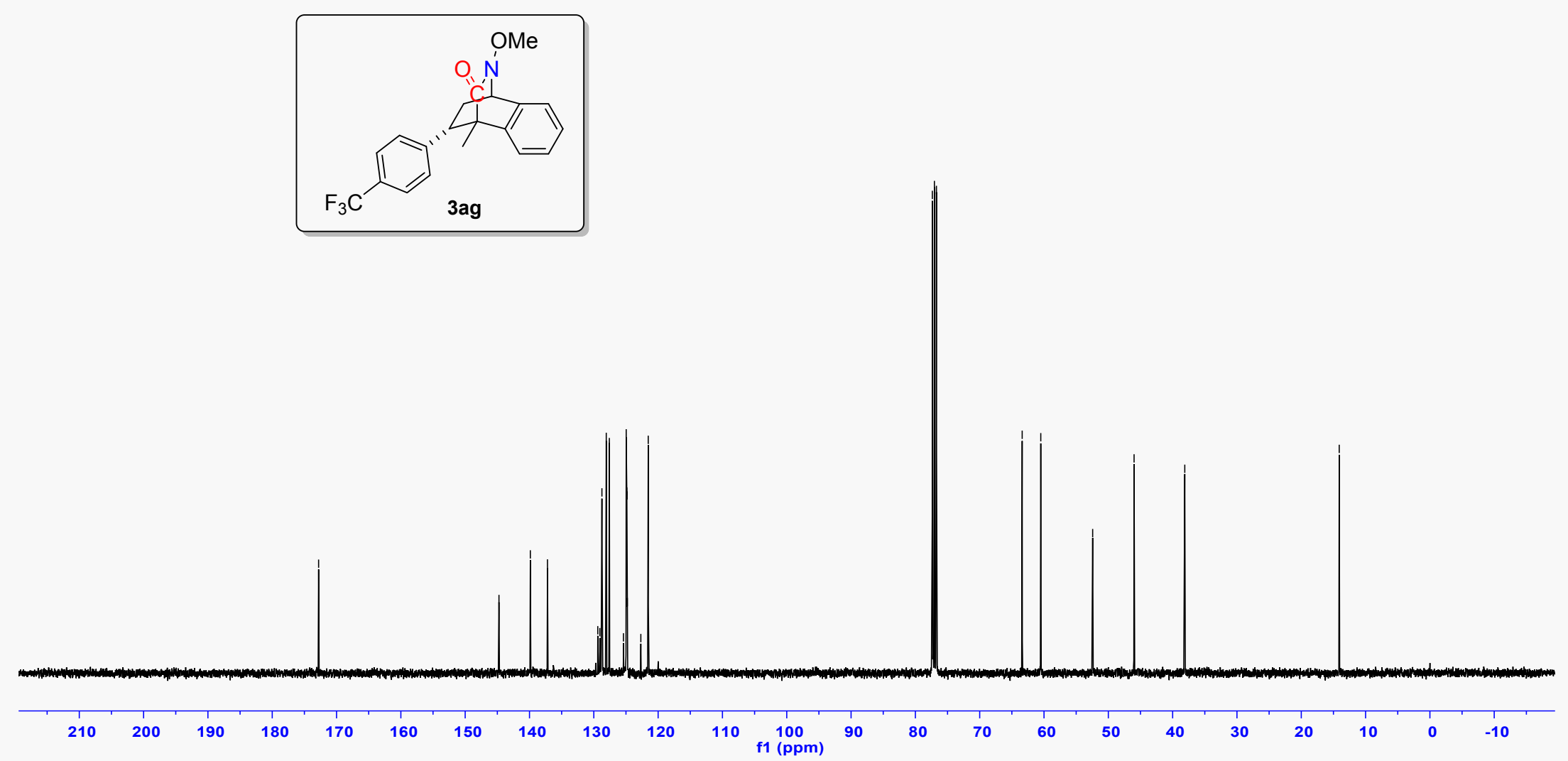




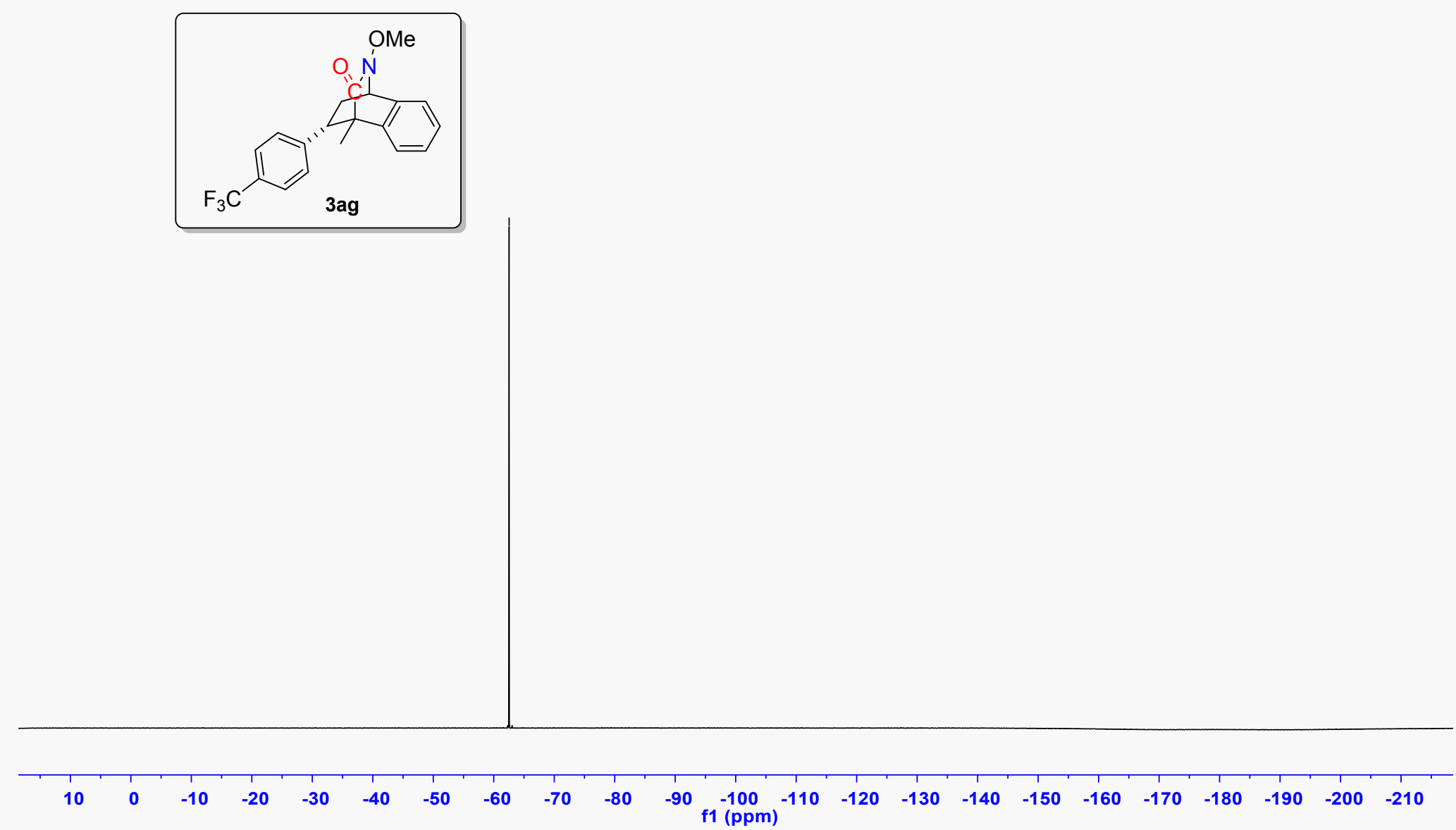




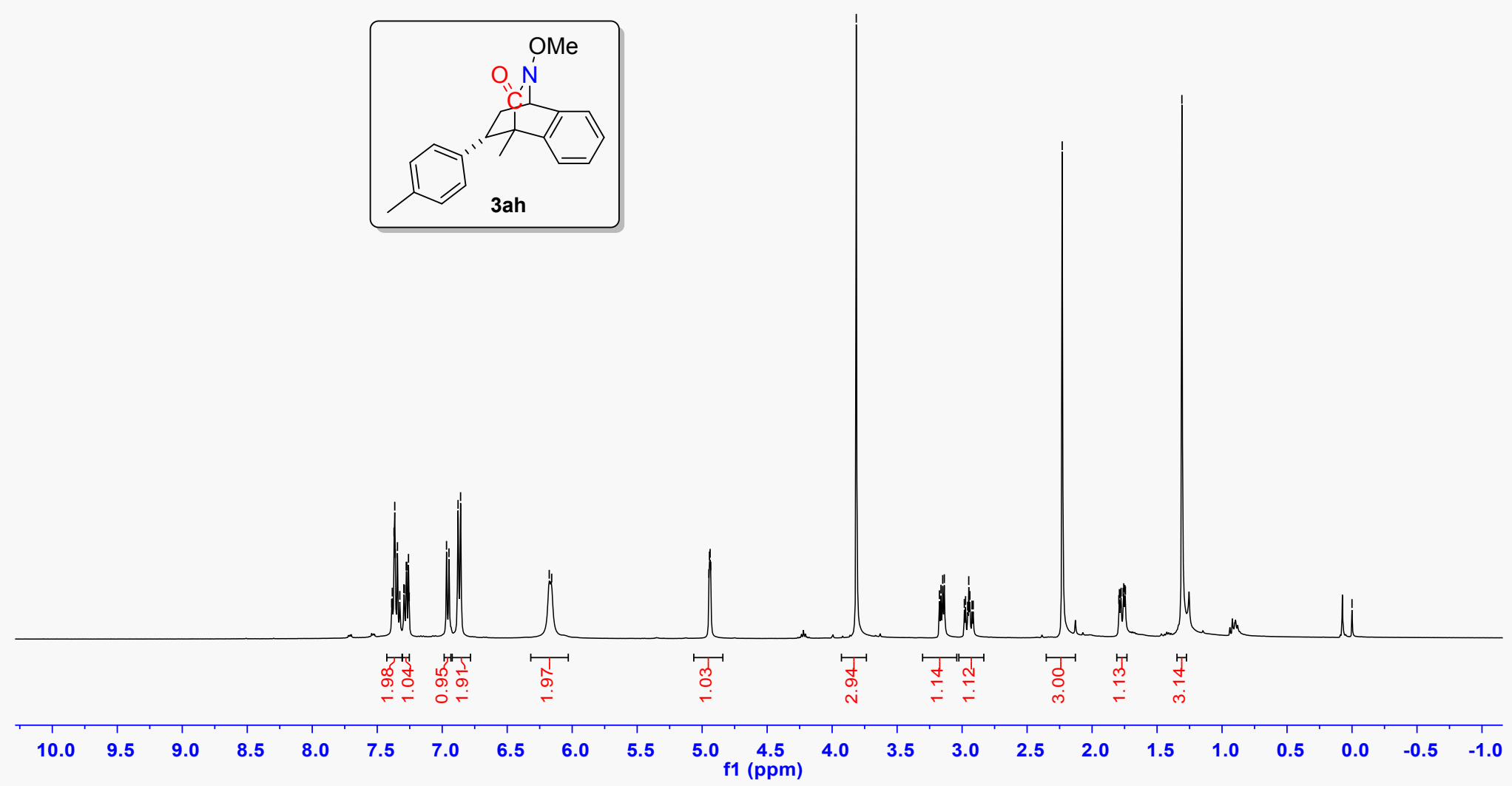


3ah-C (100 MHz, CDCl3)

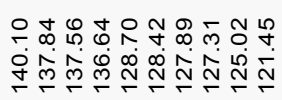

证
ริํㅡㅇ

采占

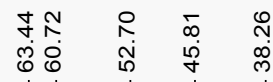

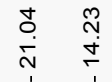
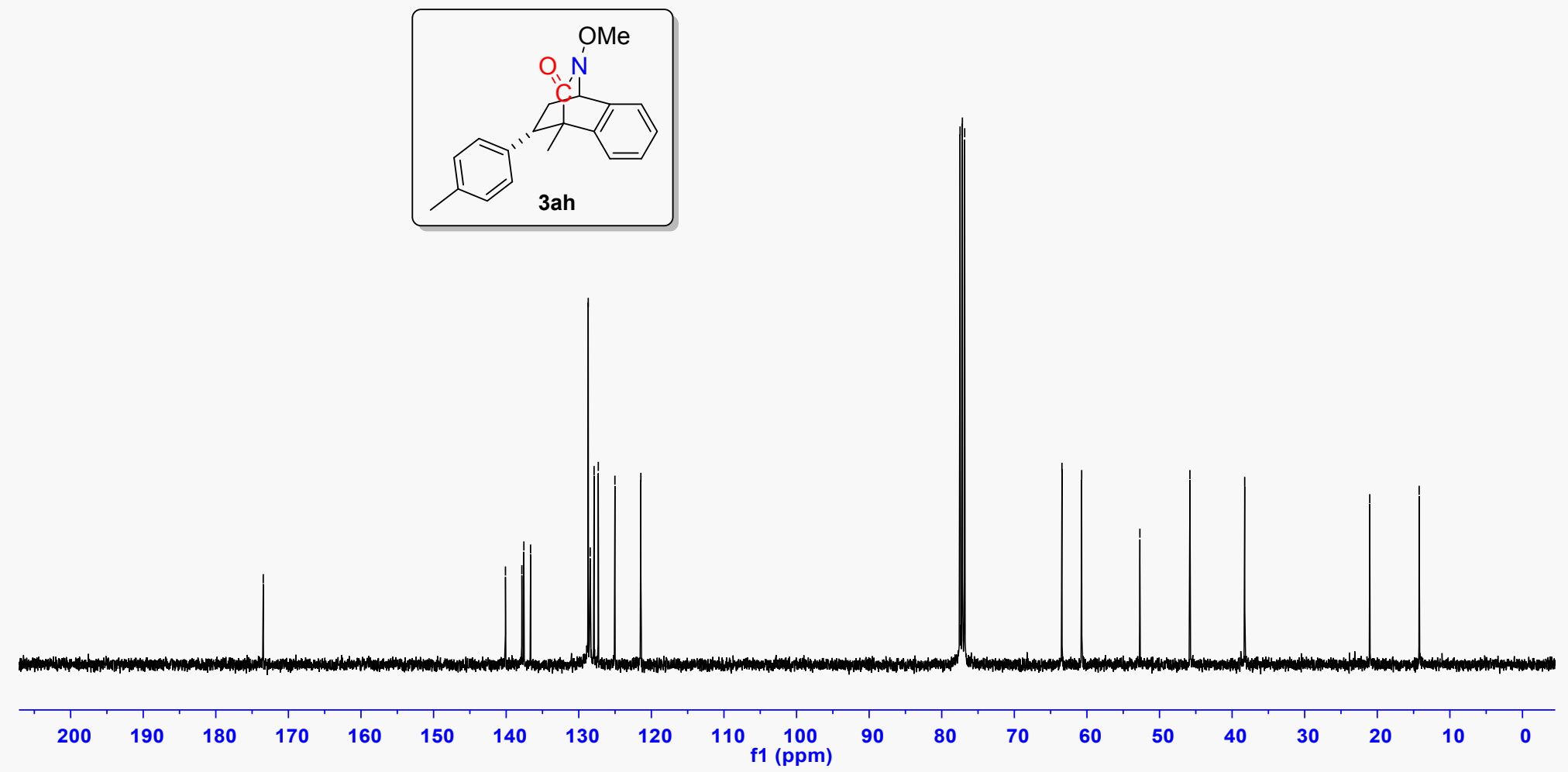

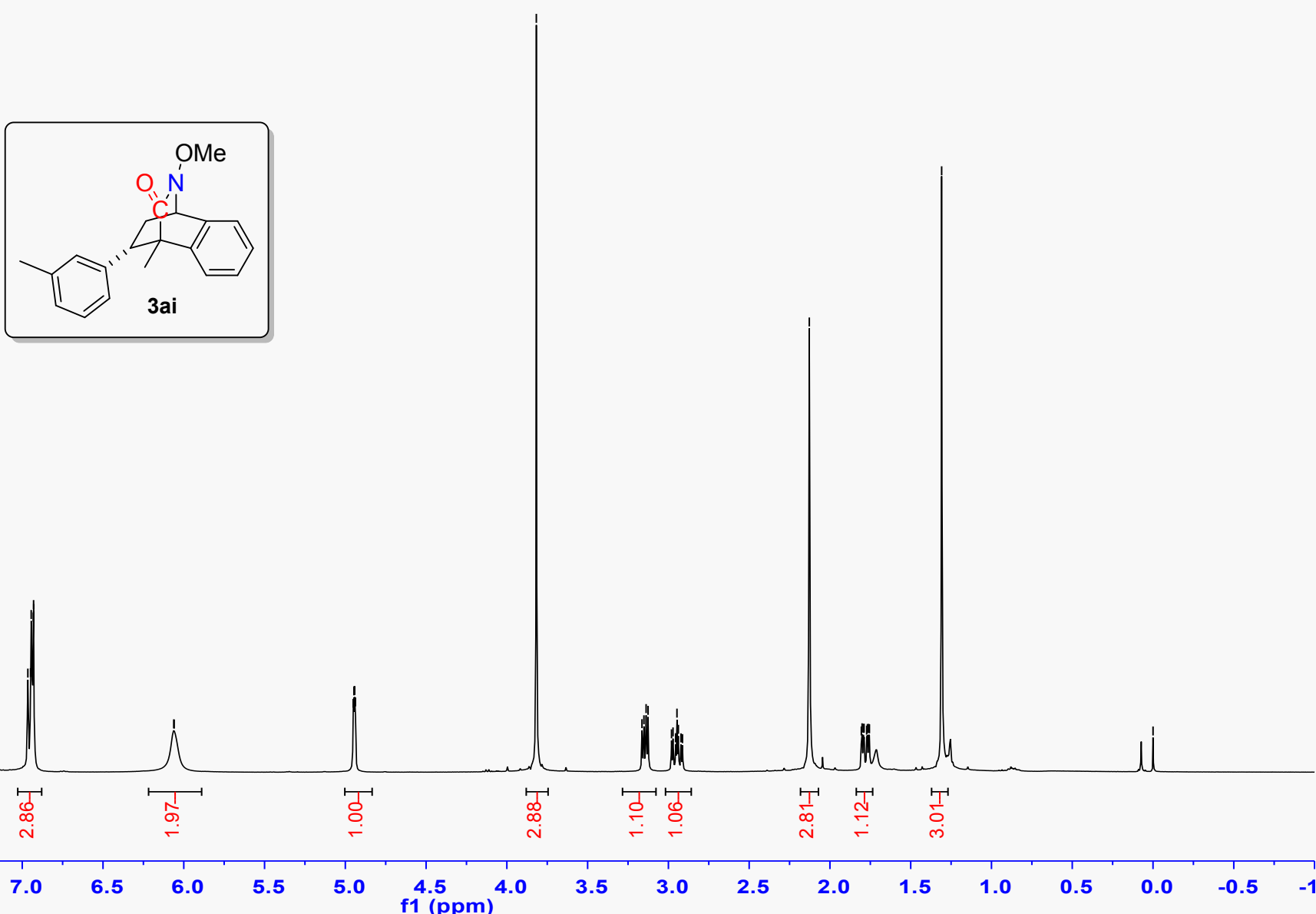
3ai-C (100 MHz, CDCl3)

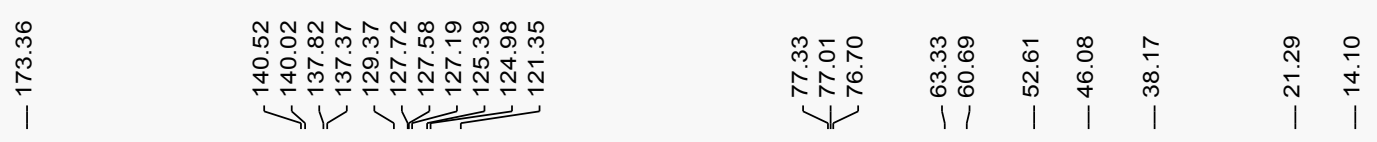

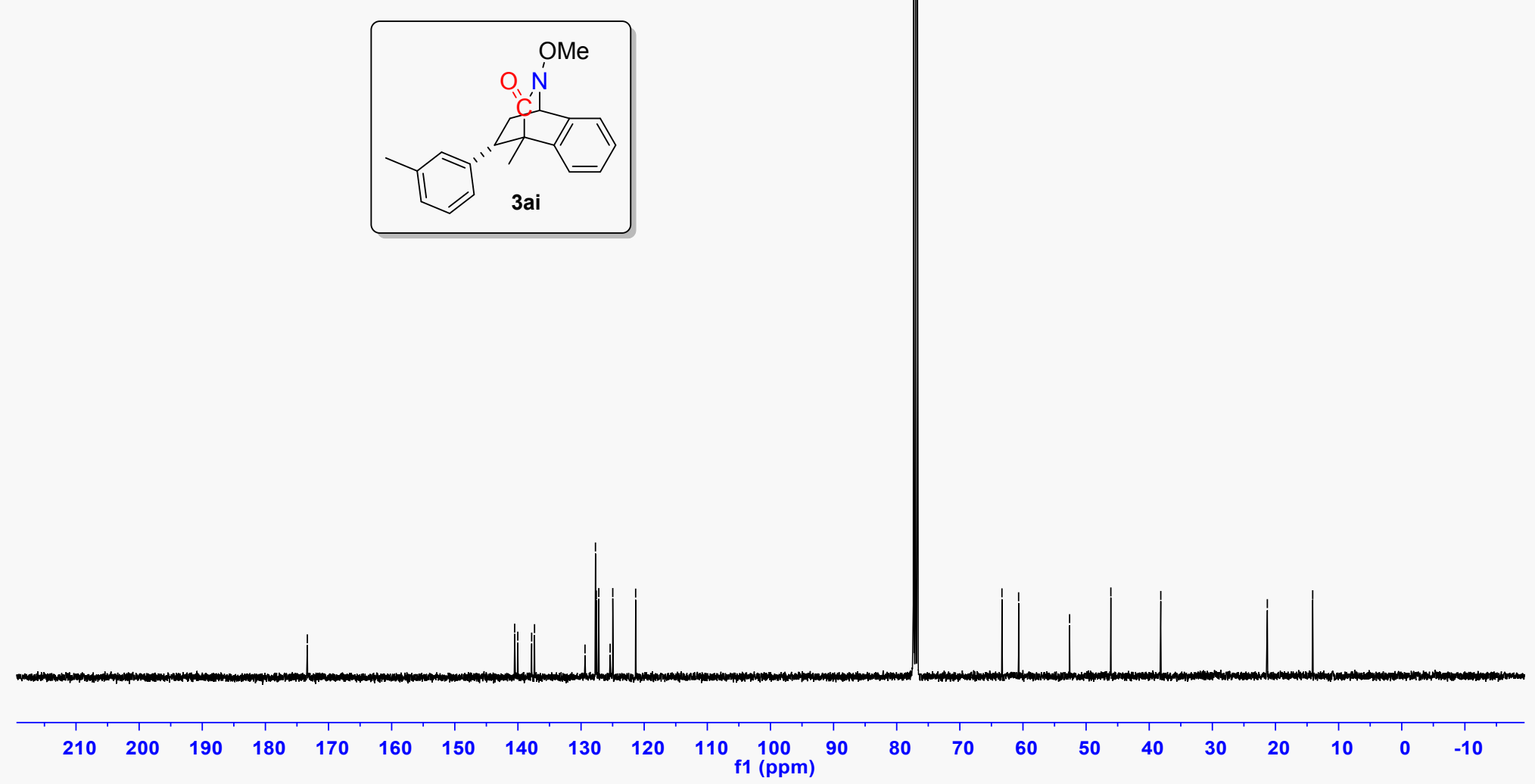

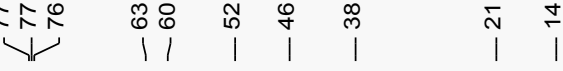


3ај-H (400 MHz, CDCl3)

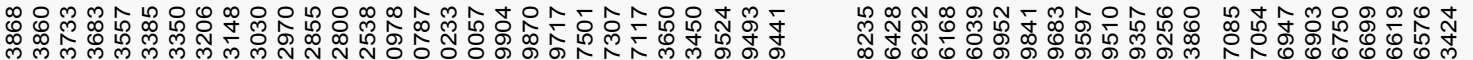

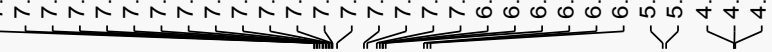

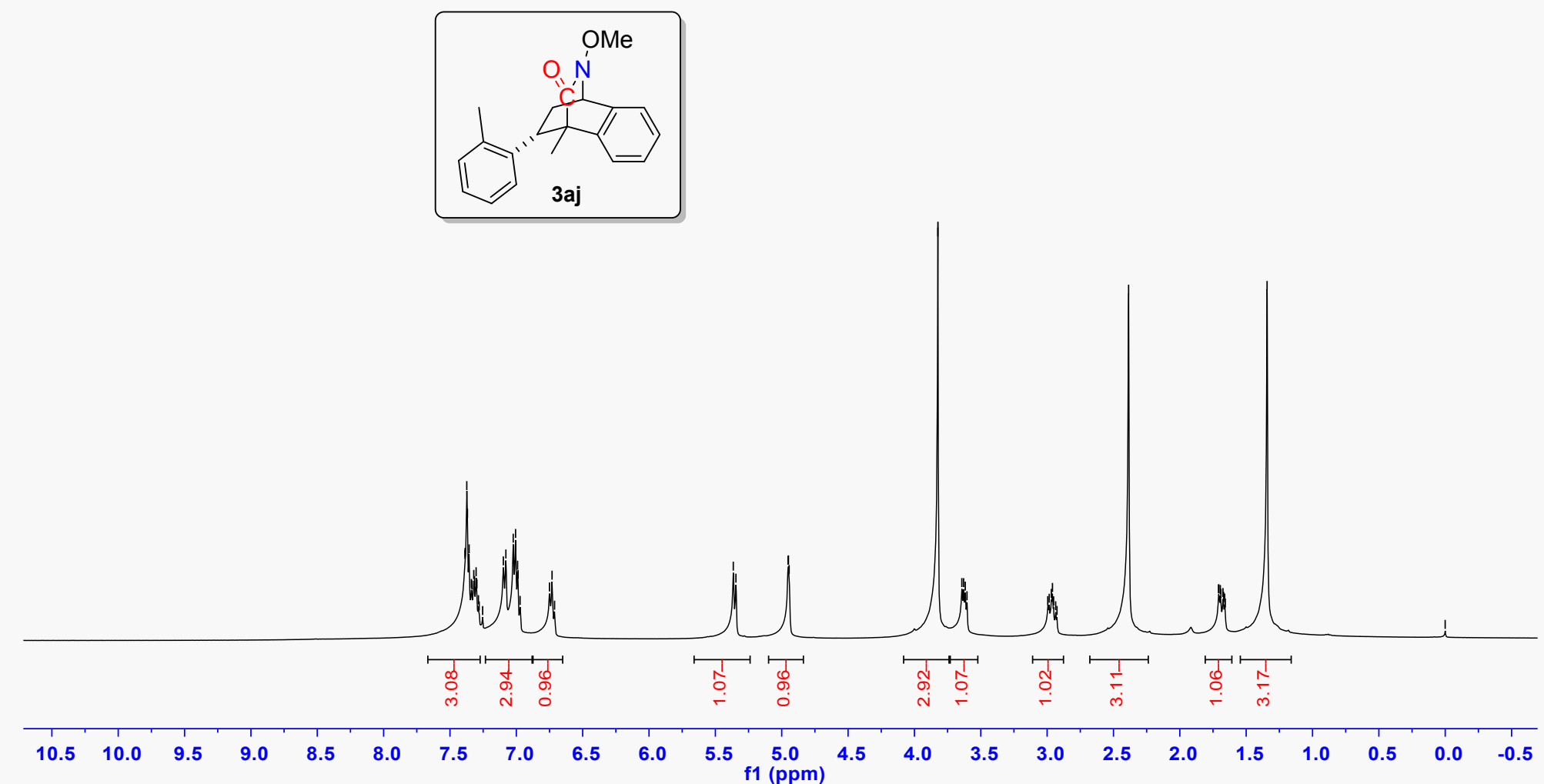


3aj-C (100 MHz, CDCl3)
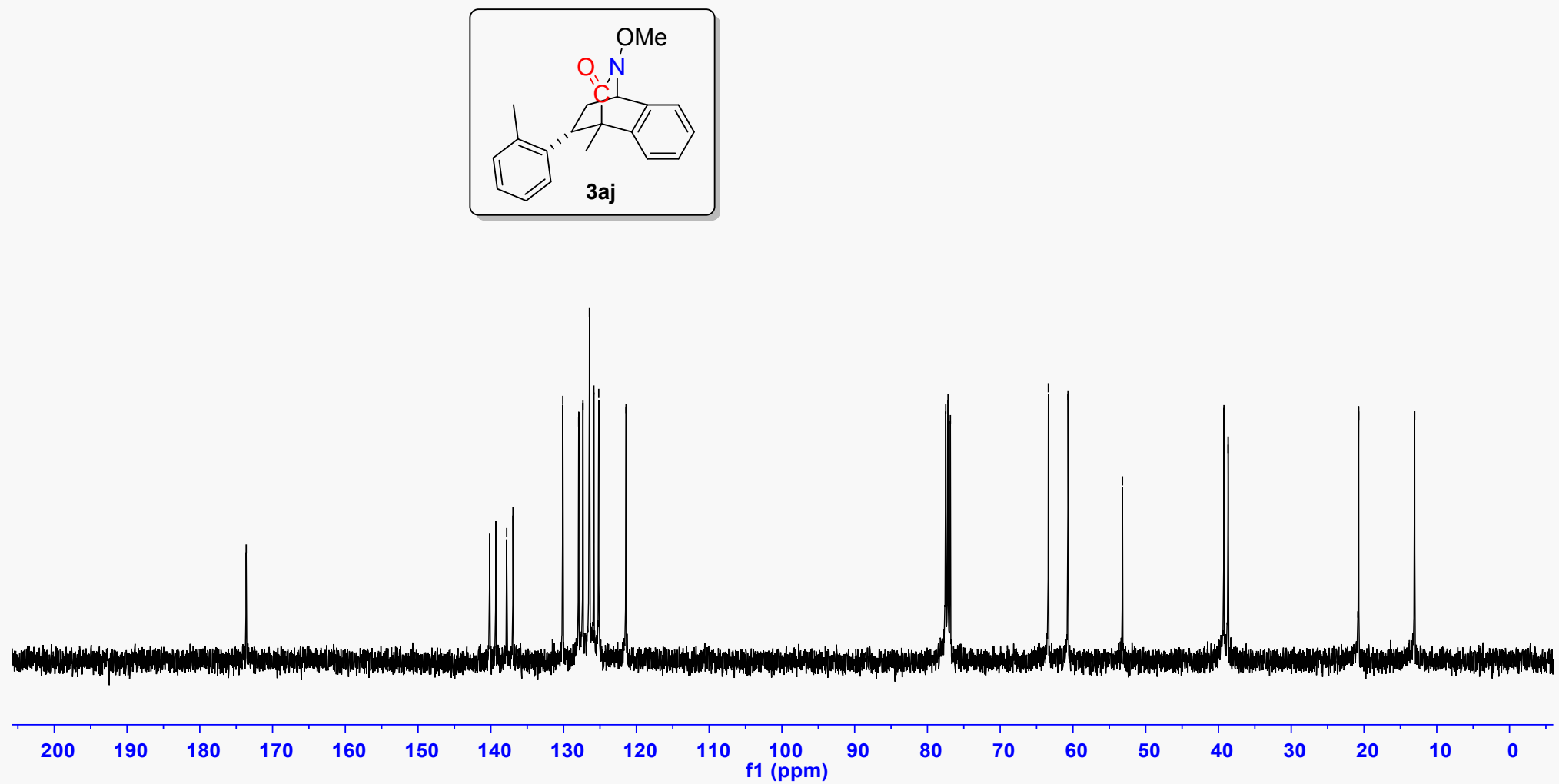
3ak-H (400 MHz, CDCl3)

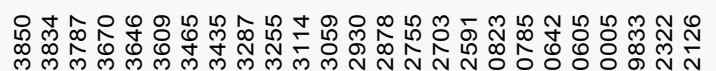

(

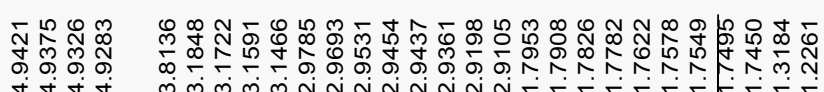

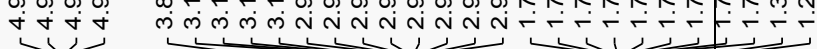

8
8
0
1

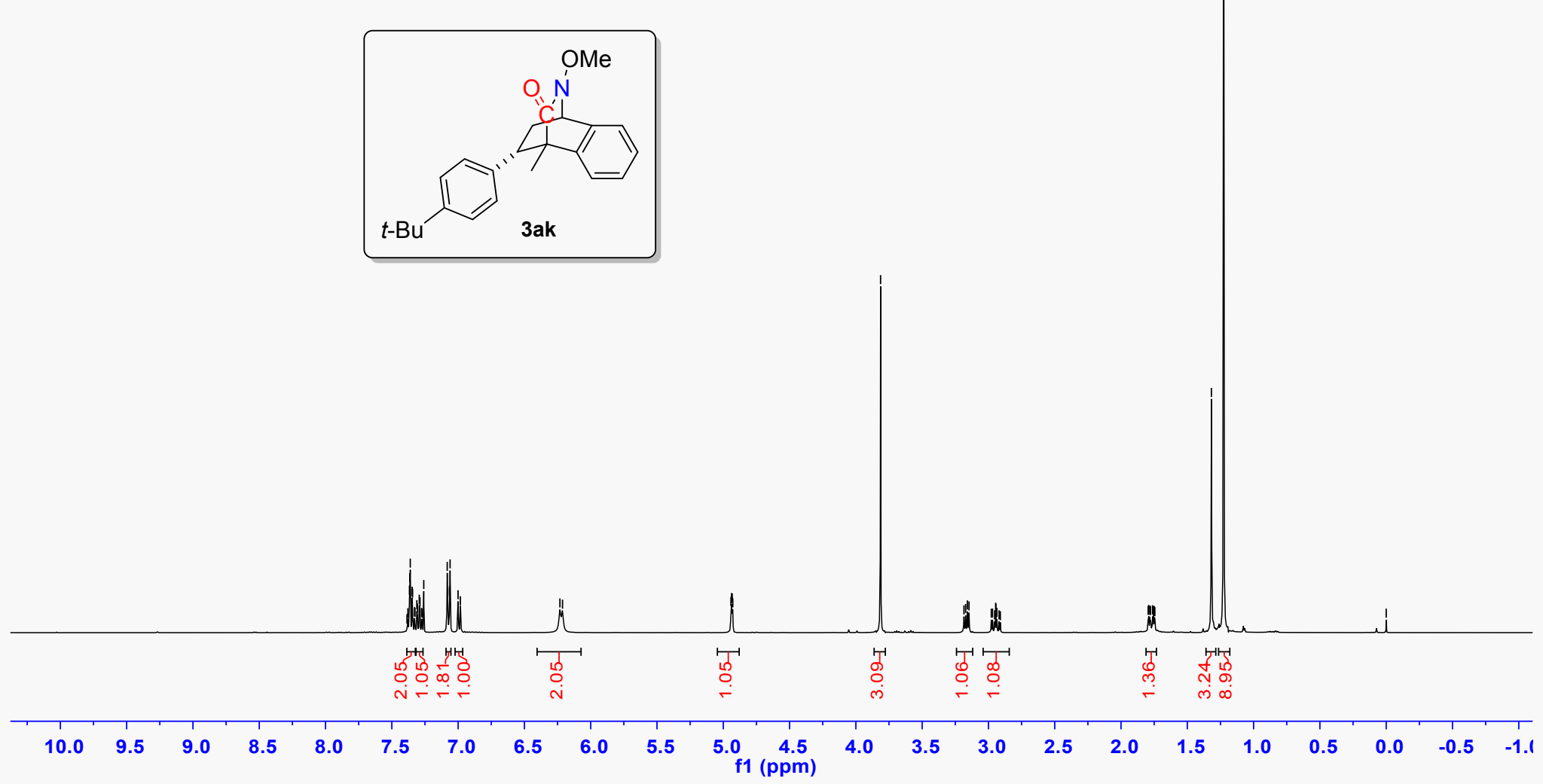


3ak-C (100 MHz, CDCl3)

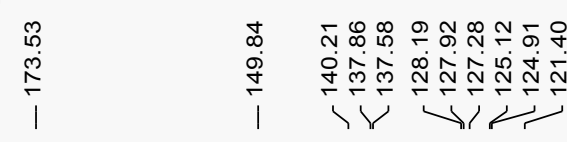

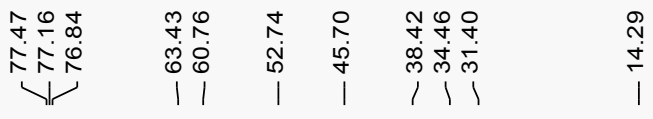

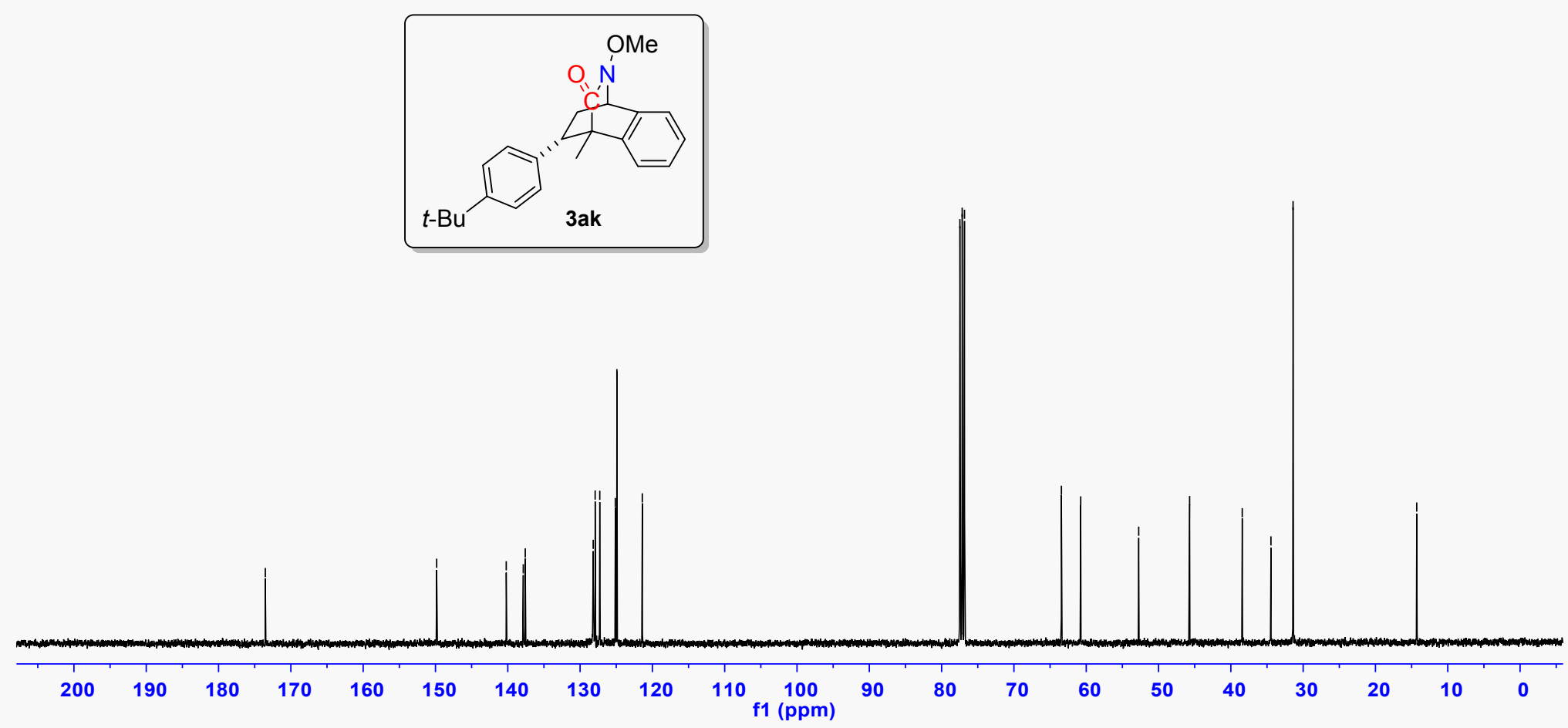


3al-H (400 MHz, CDCl3)

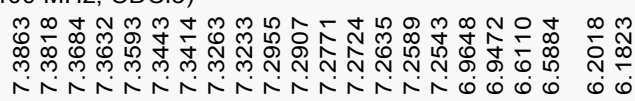

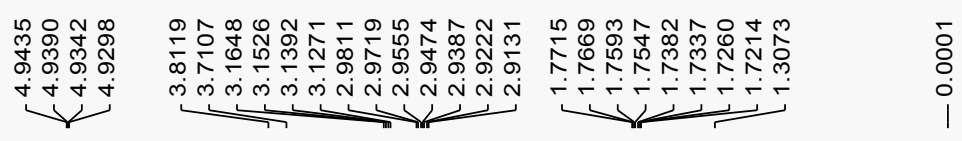

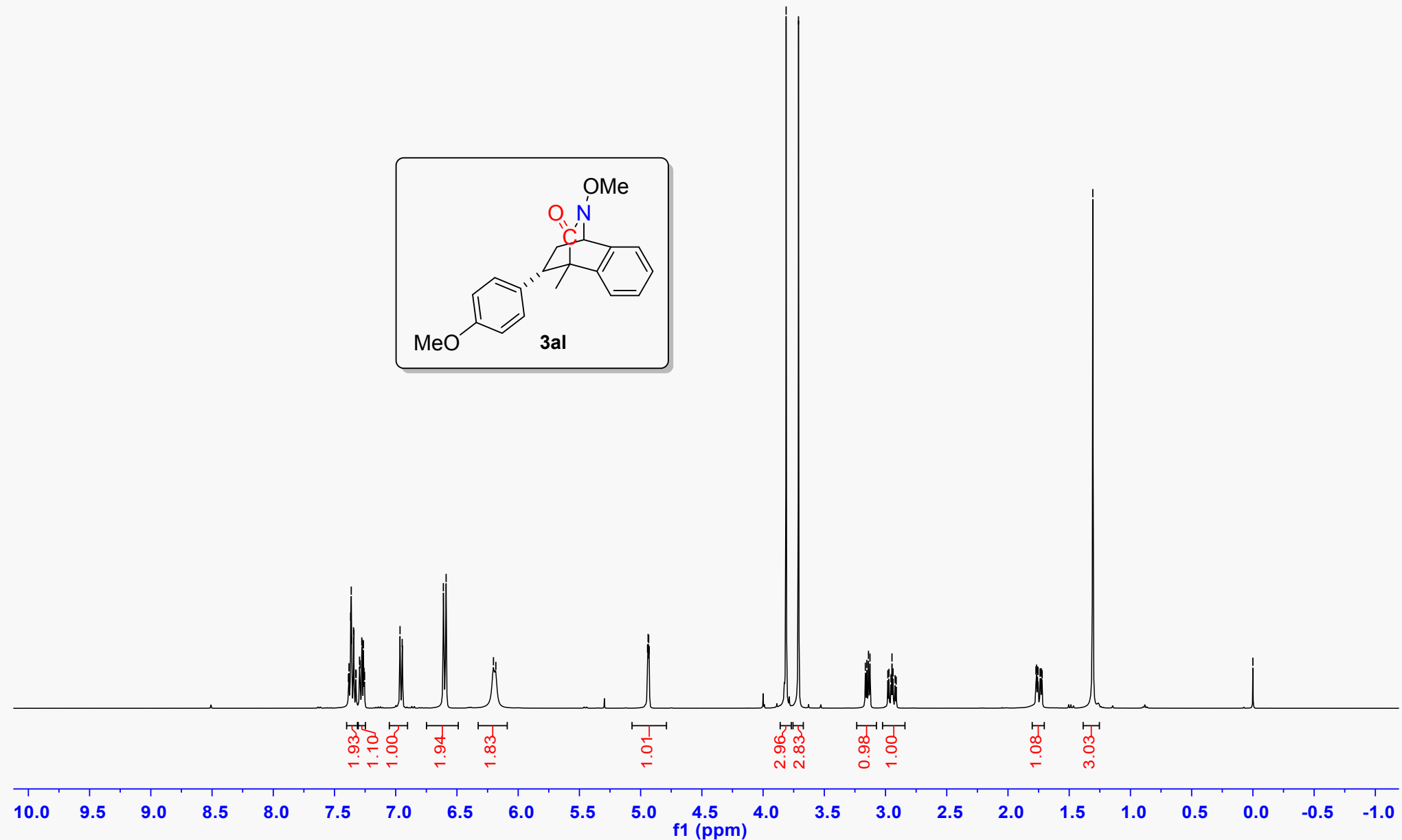


3al-C (100 MHz, CDCl3)
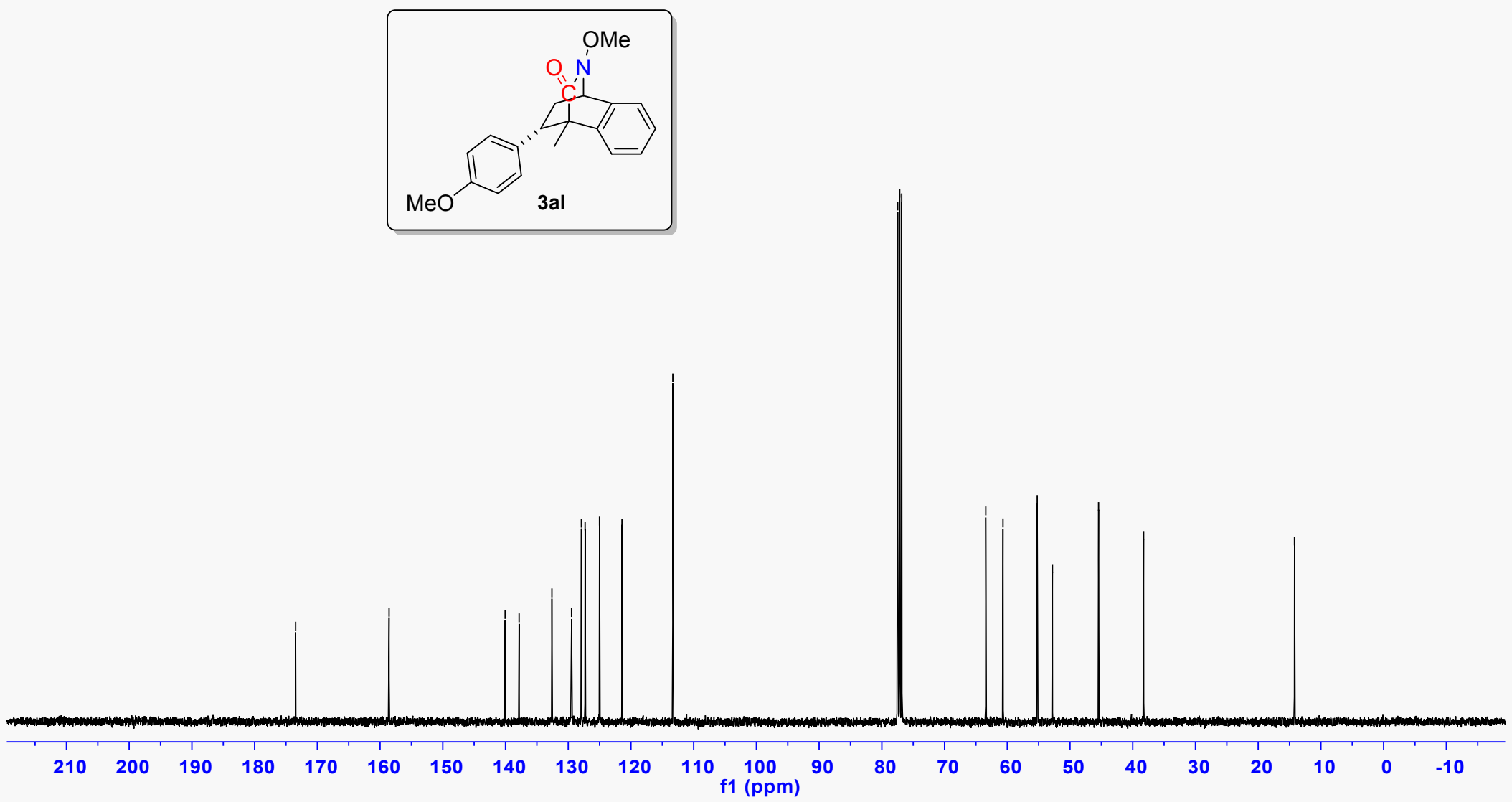
$3 \mathrm{am}-\mathrm{H}(400 \mathrm{MHz}, \mathrm{CDCl} 3)$

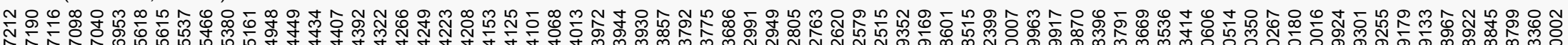

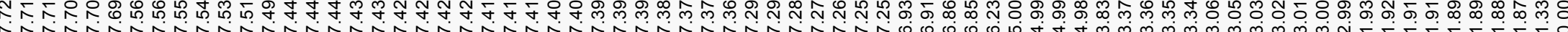

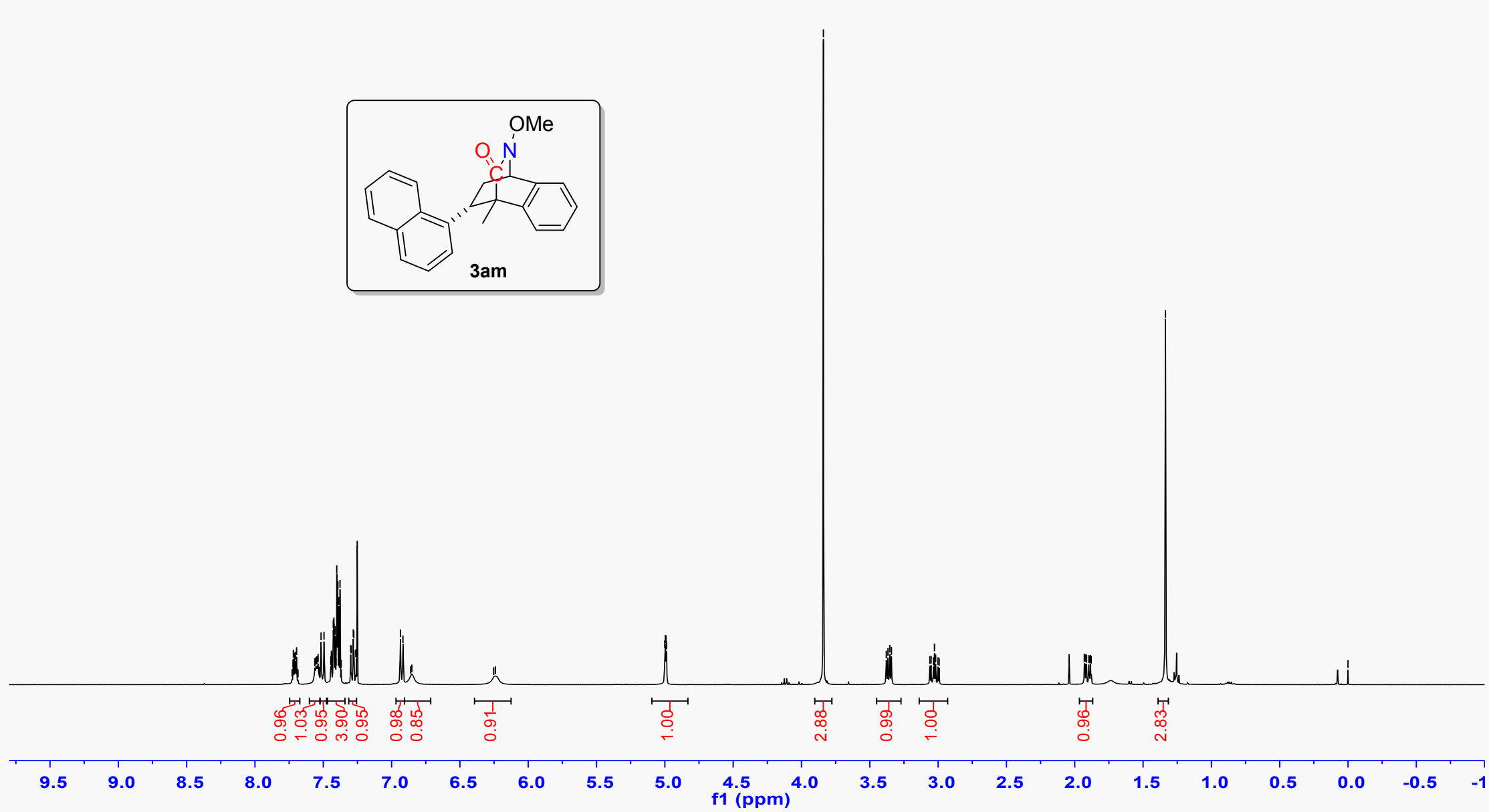




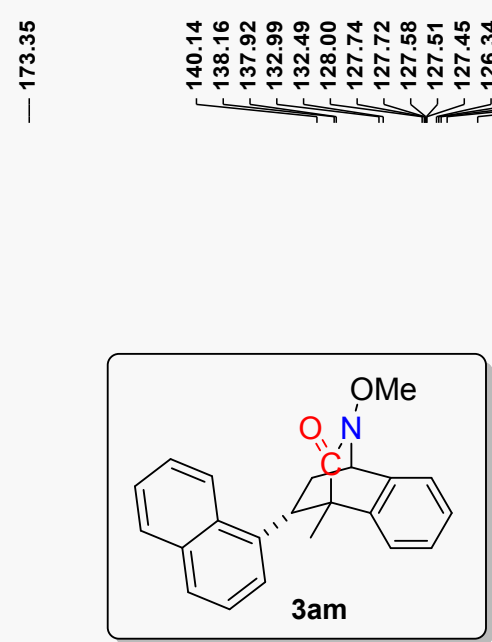

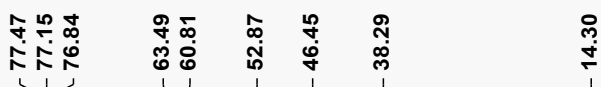

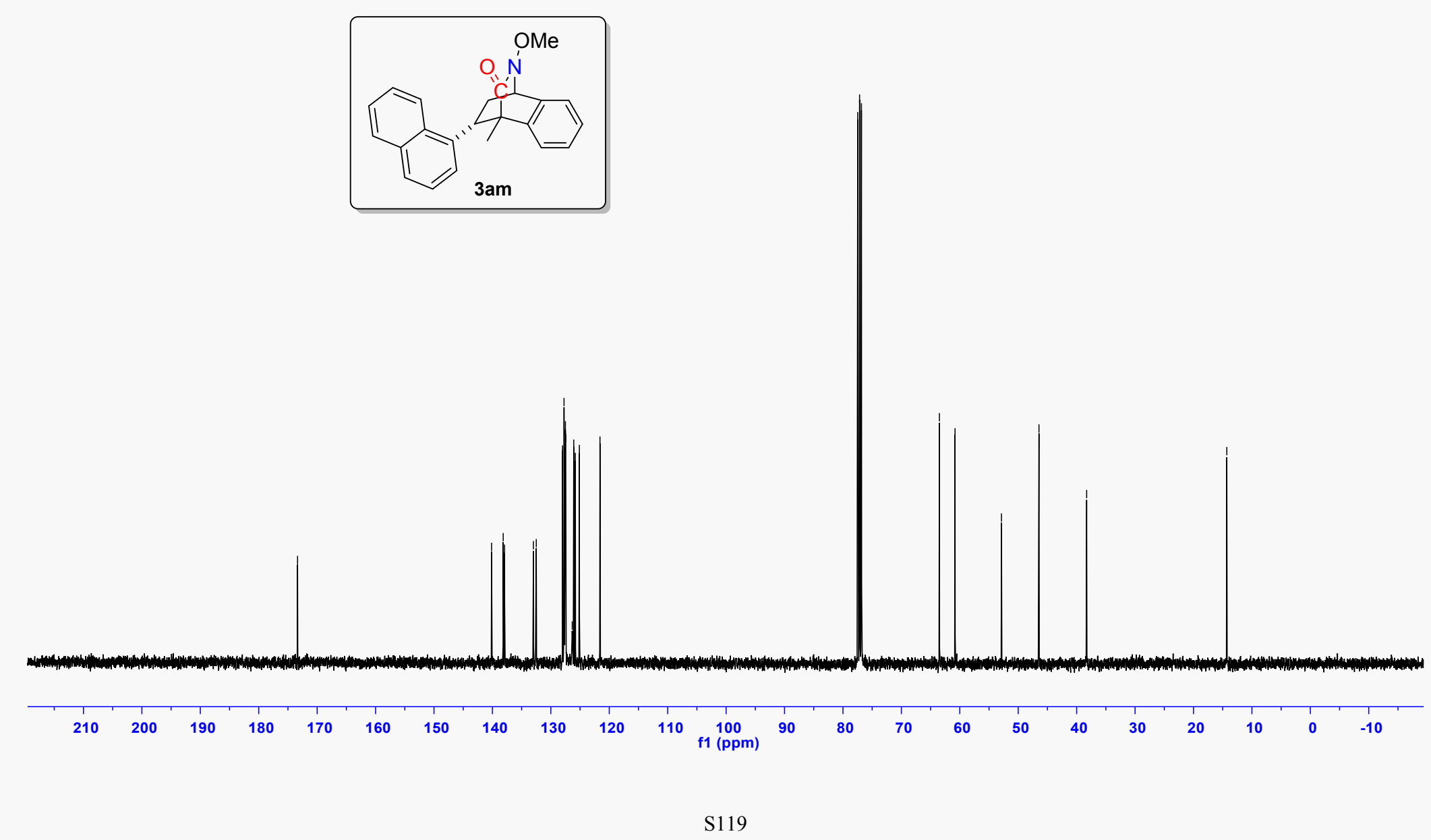




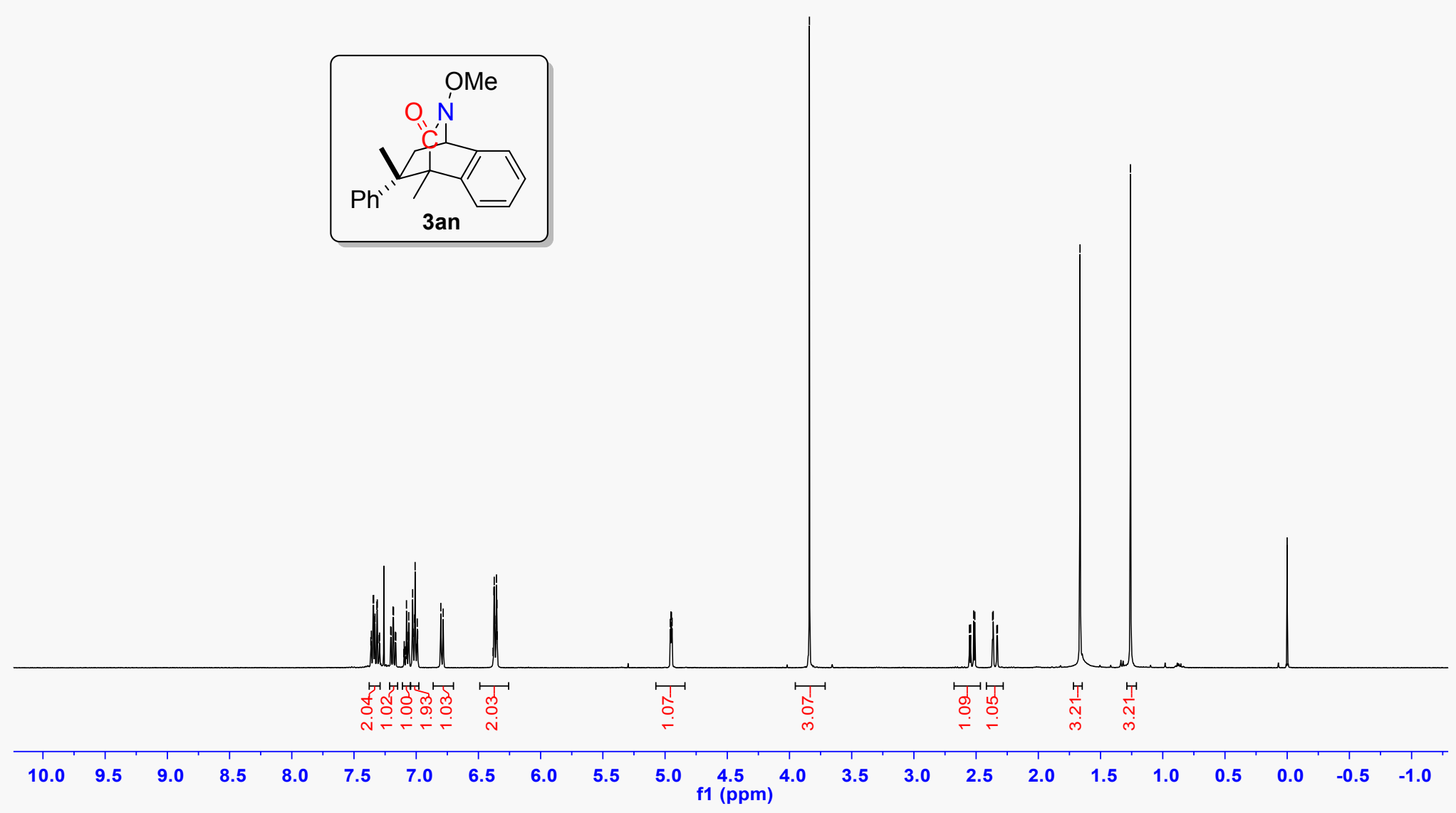


3an-C (100 MHz, CDCl3)

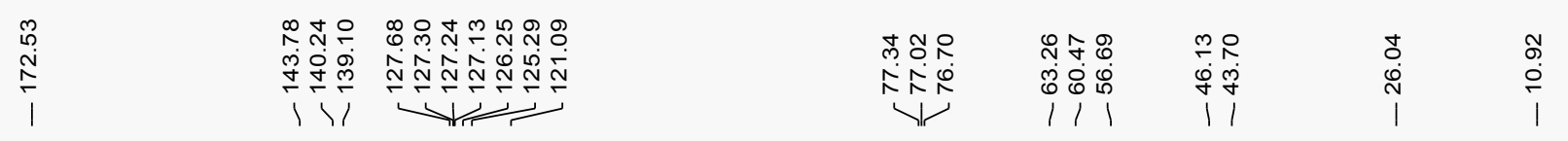
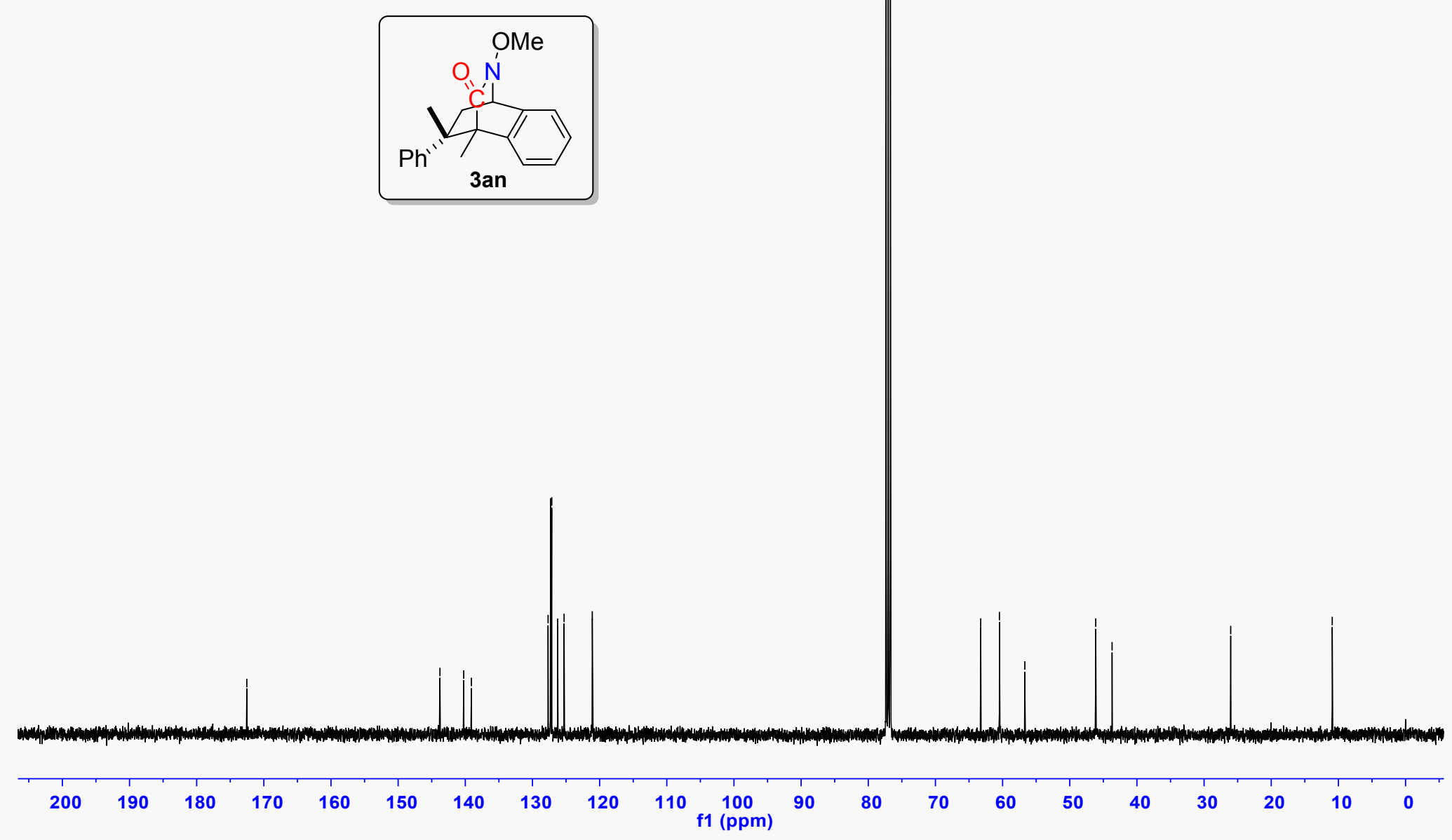


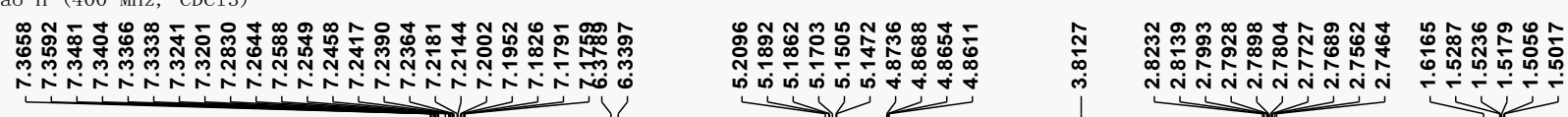
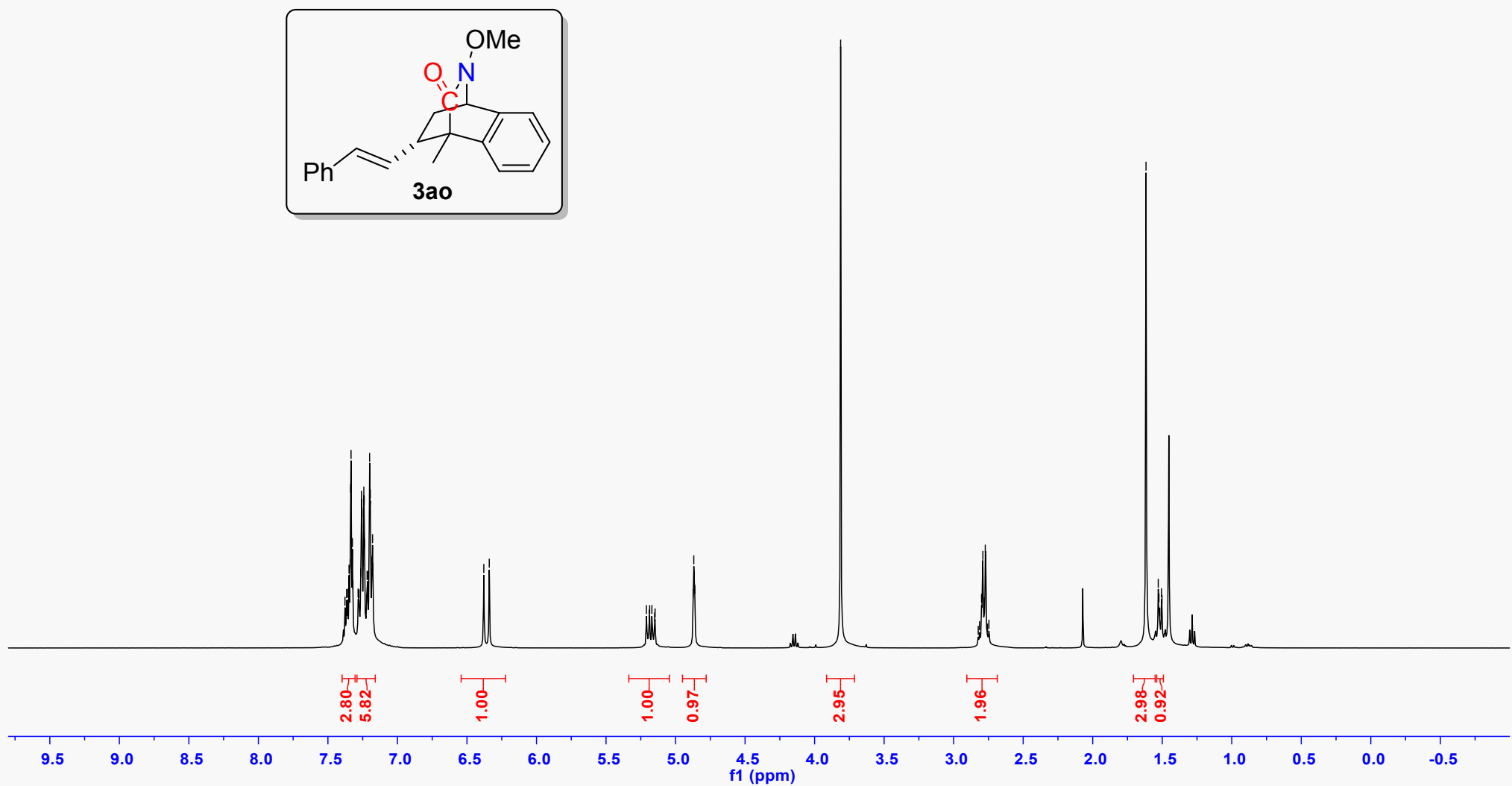
3ao-C (100 MHz, CDC13)

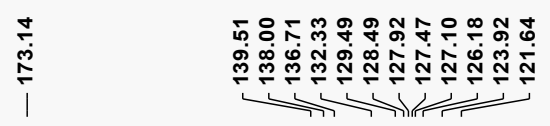

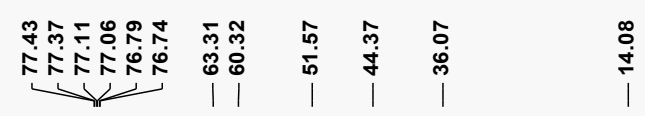
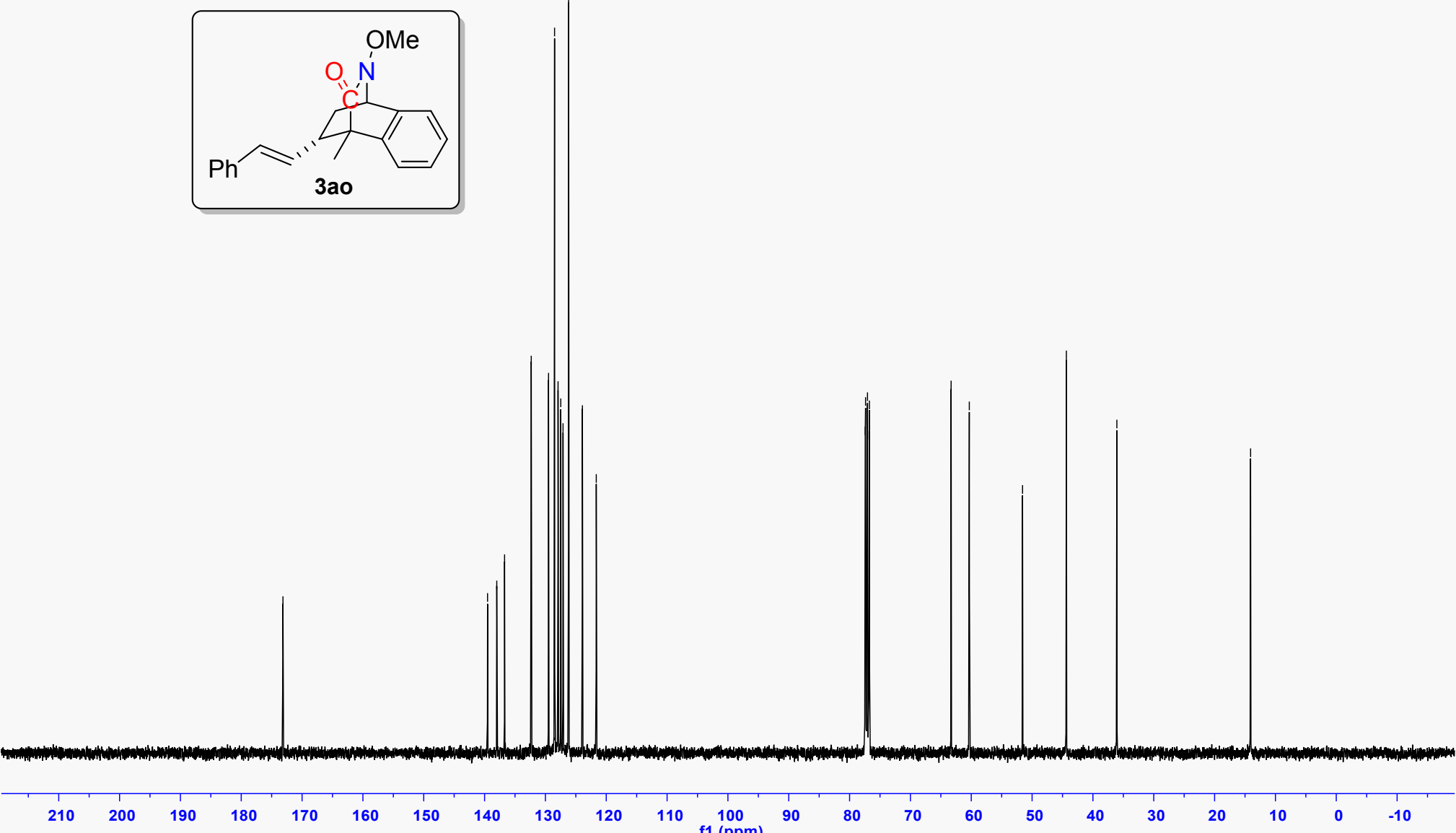


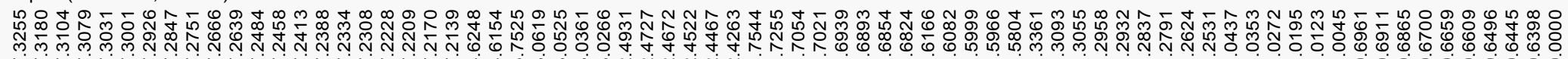

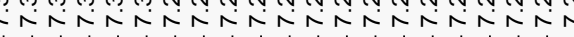

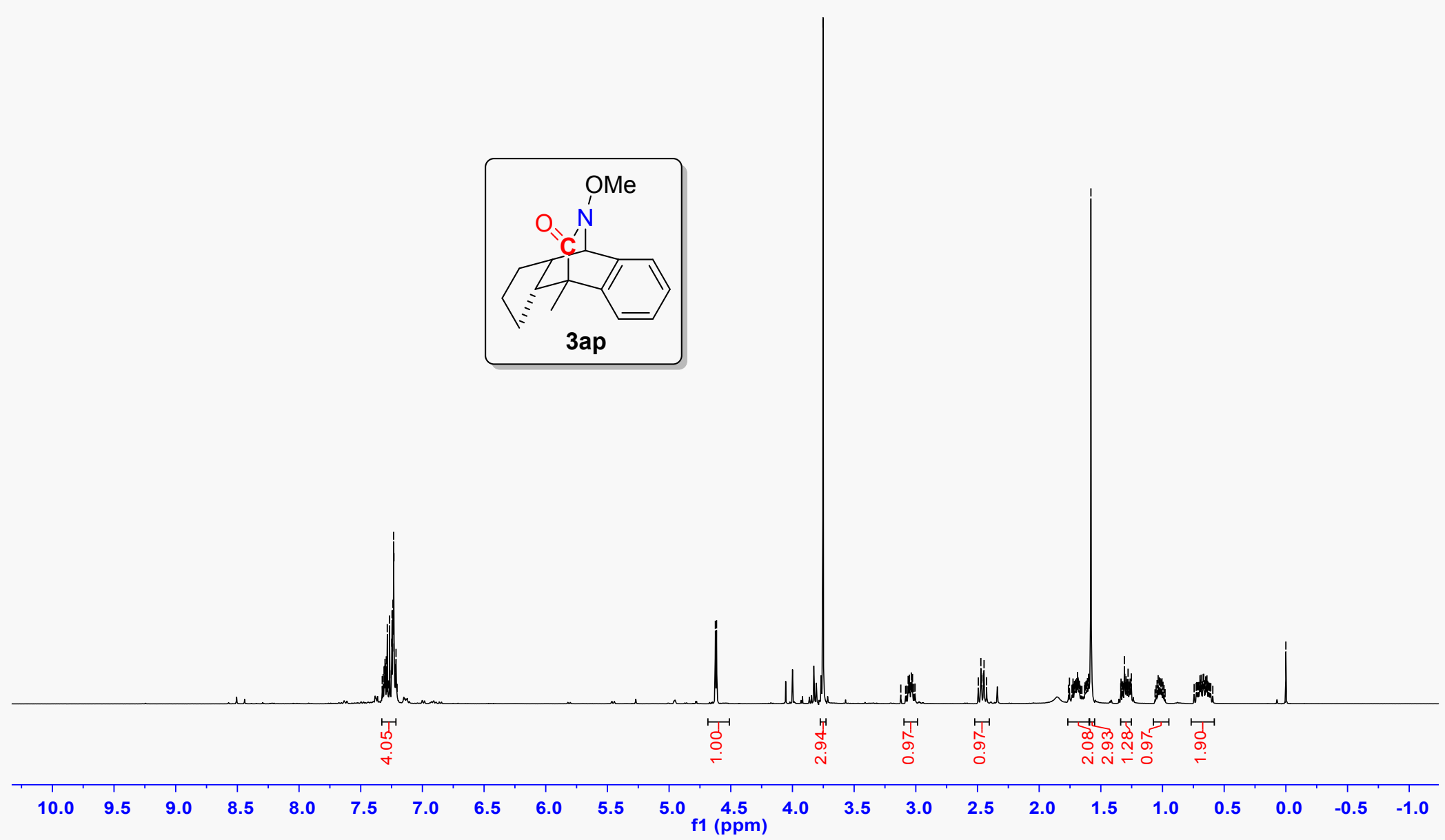


3ap-C (100 MHz, CDCl3)
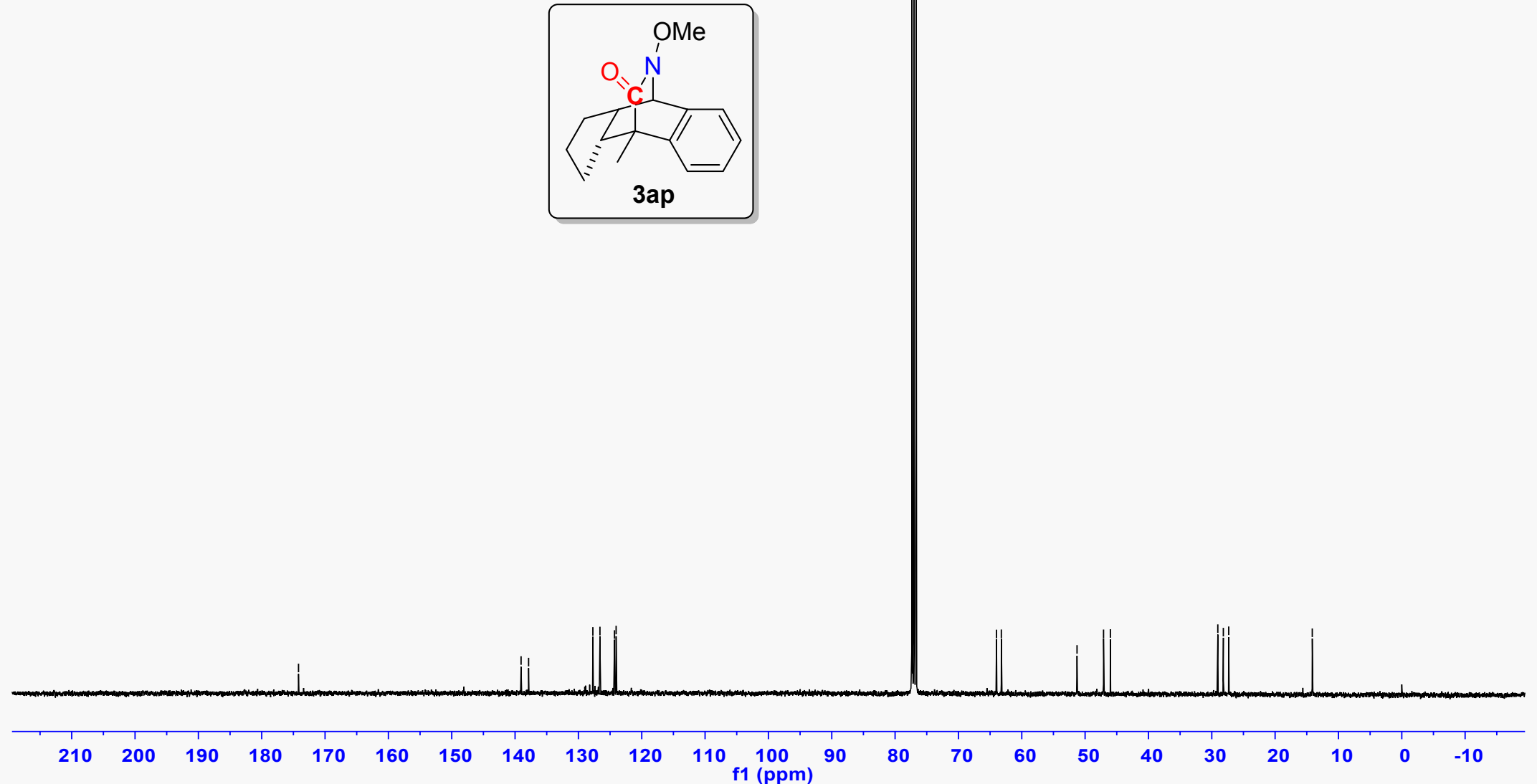


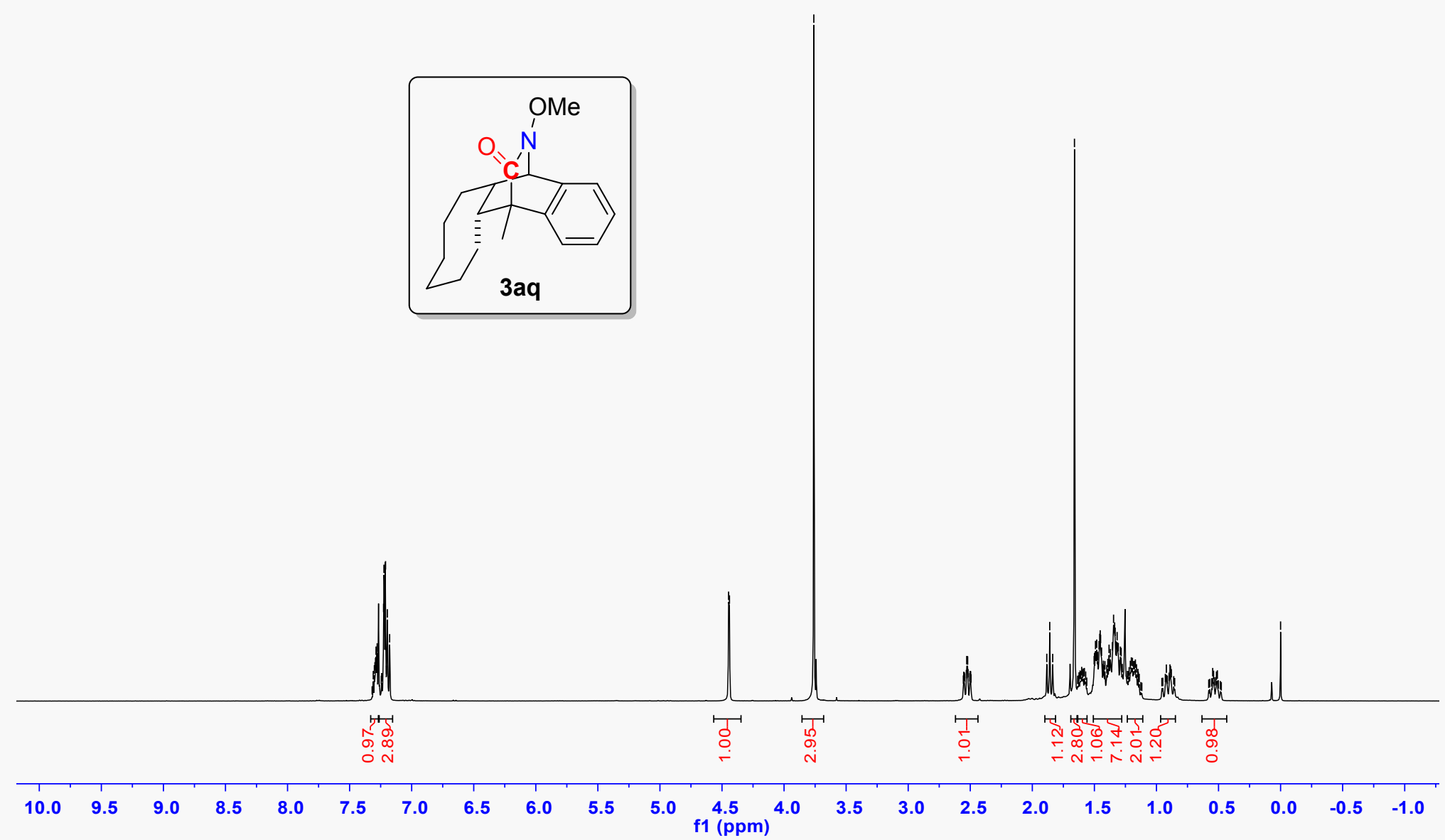


3aq-C (100 MHz, CDCl3)

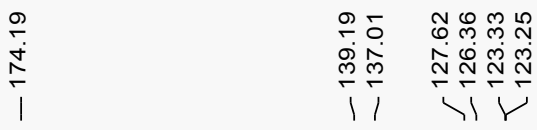

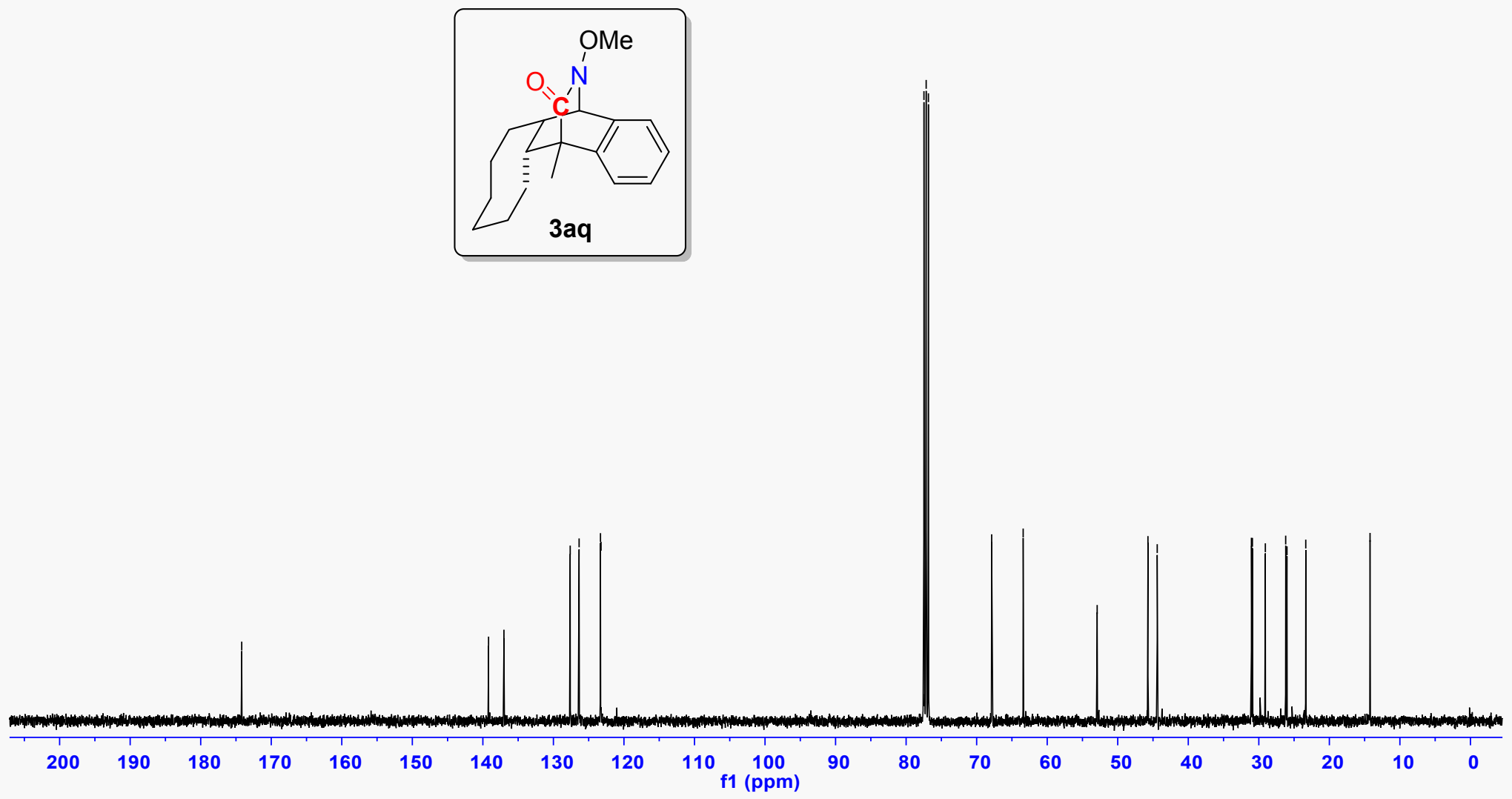

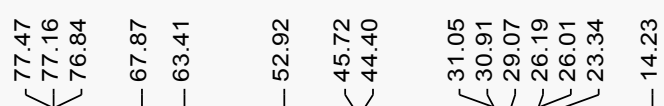


4a- $\mathrm{H}(400 \mathrm{MHz}, \mathrm{CDCl} 3)$

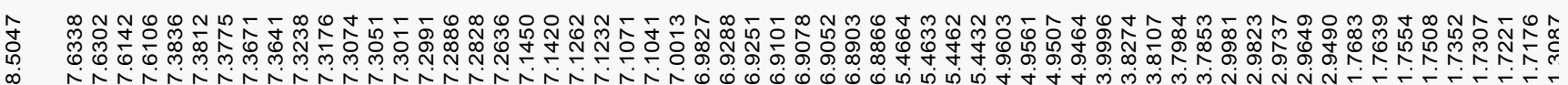

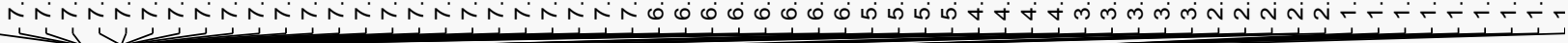

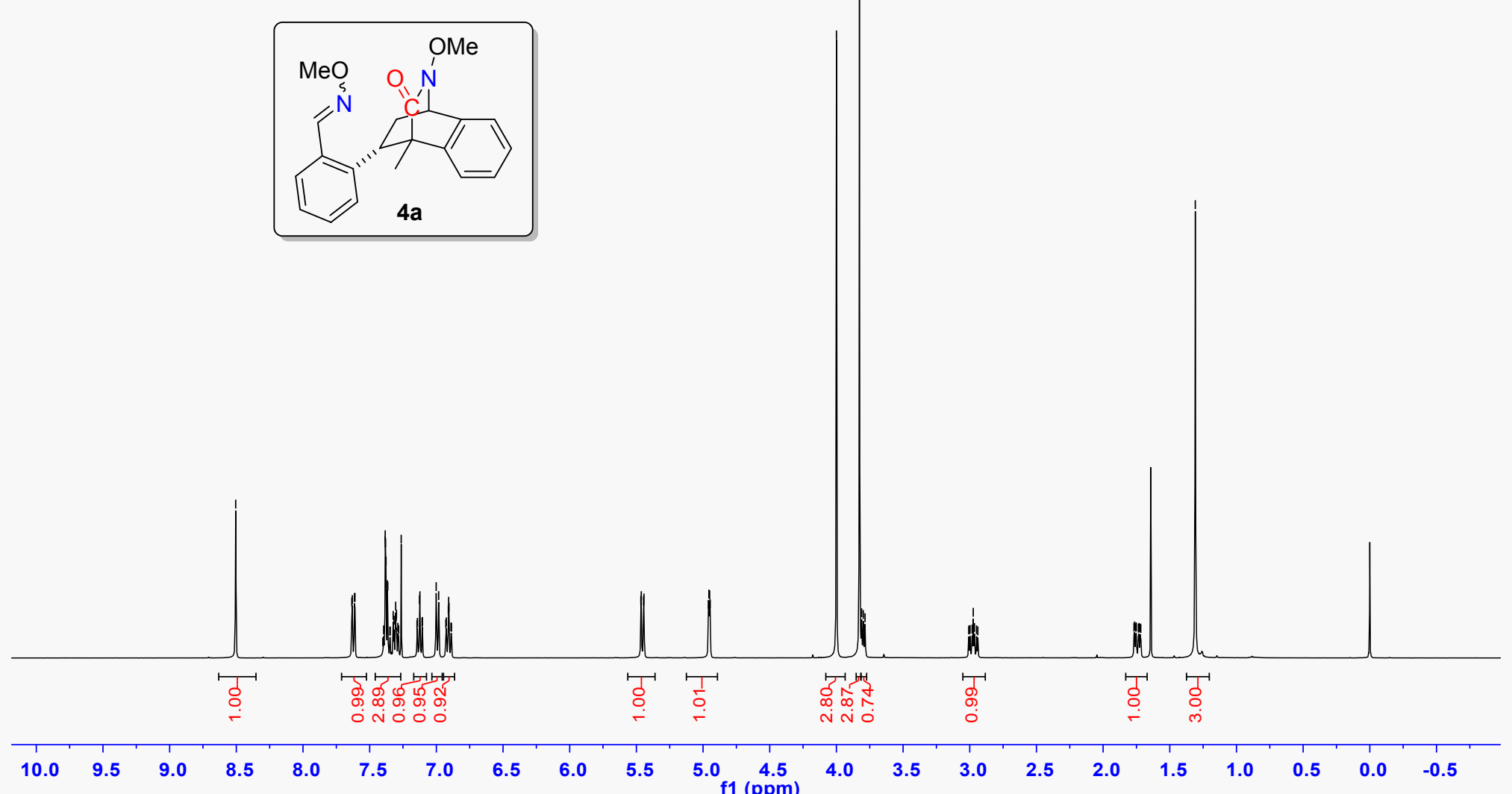


4a-C (100 MHz, CDCl3)

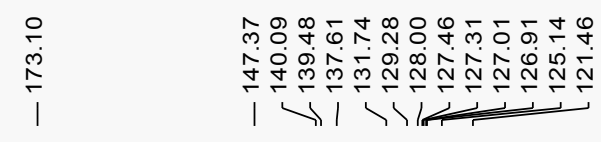

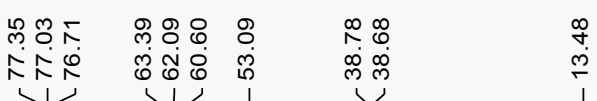
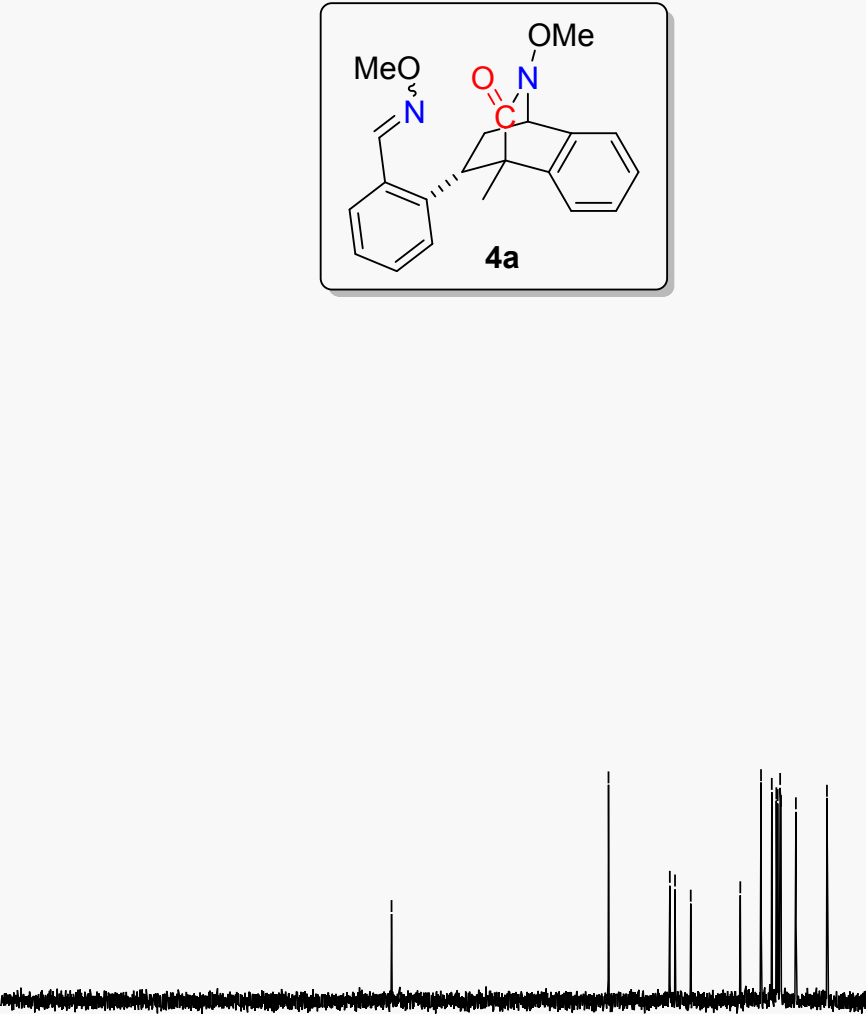

$\begin{array}{lllllllllllll}210 & 200 & 190 & 180 & 170 & 160 & 150 & 140 & 130 & 120 & 110 & 100 & 90\end{array}$ f1 $(\mathrm{ppm})$

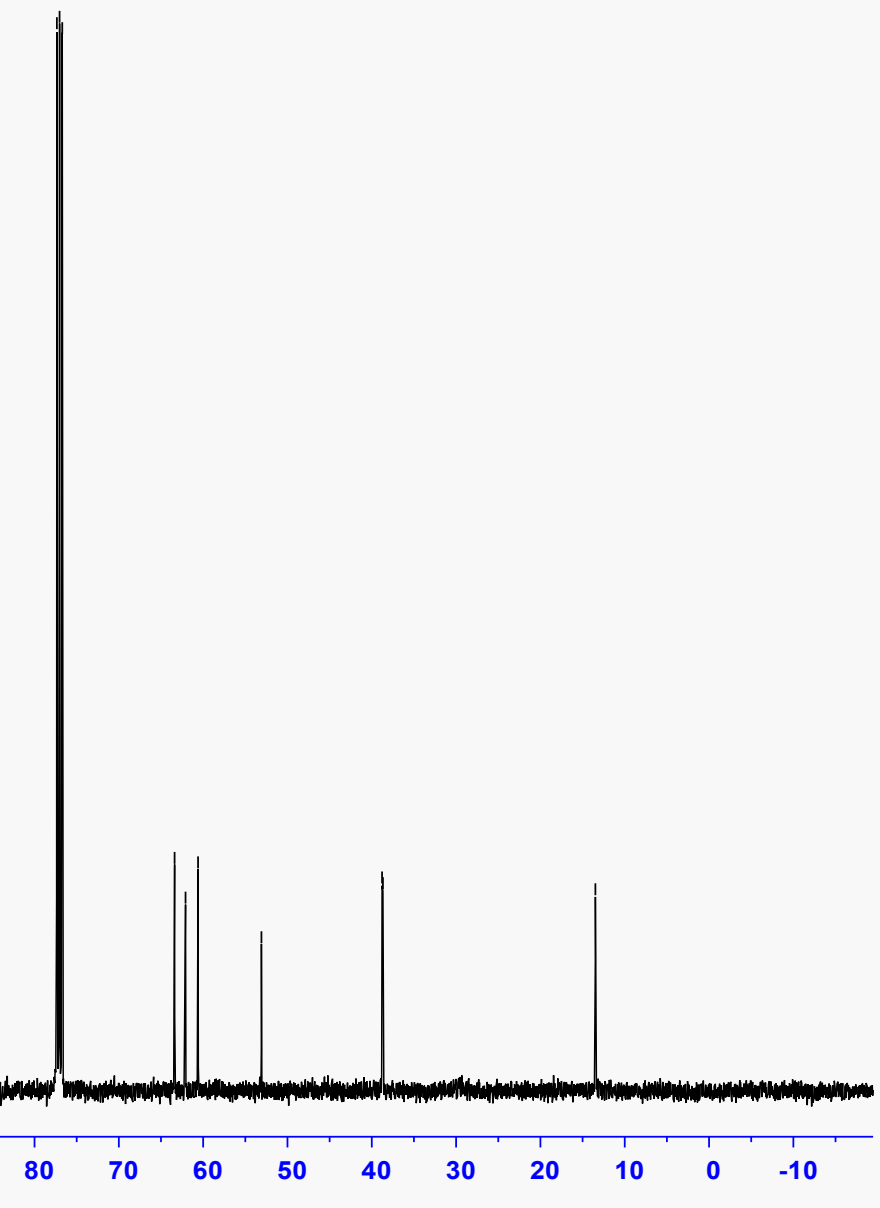




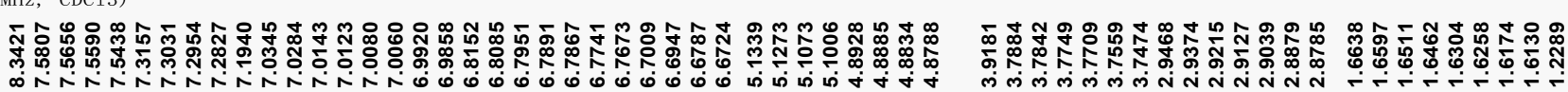

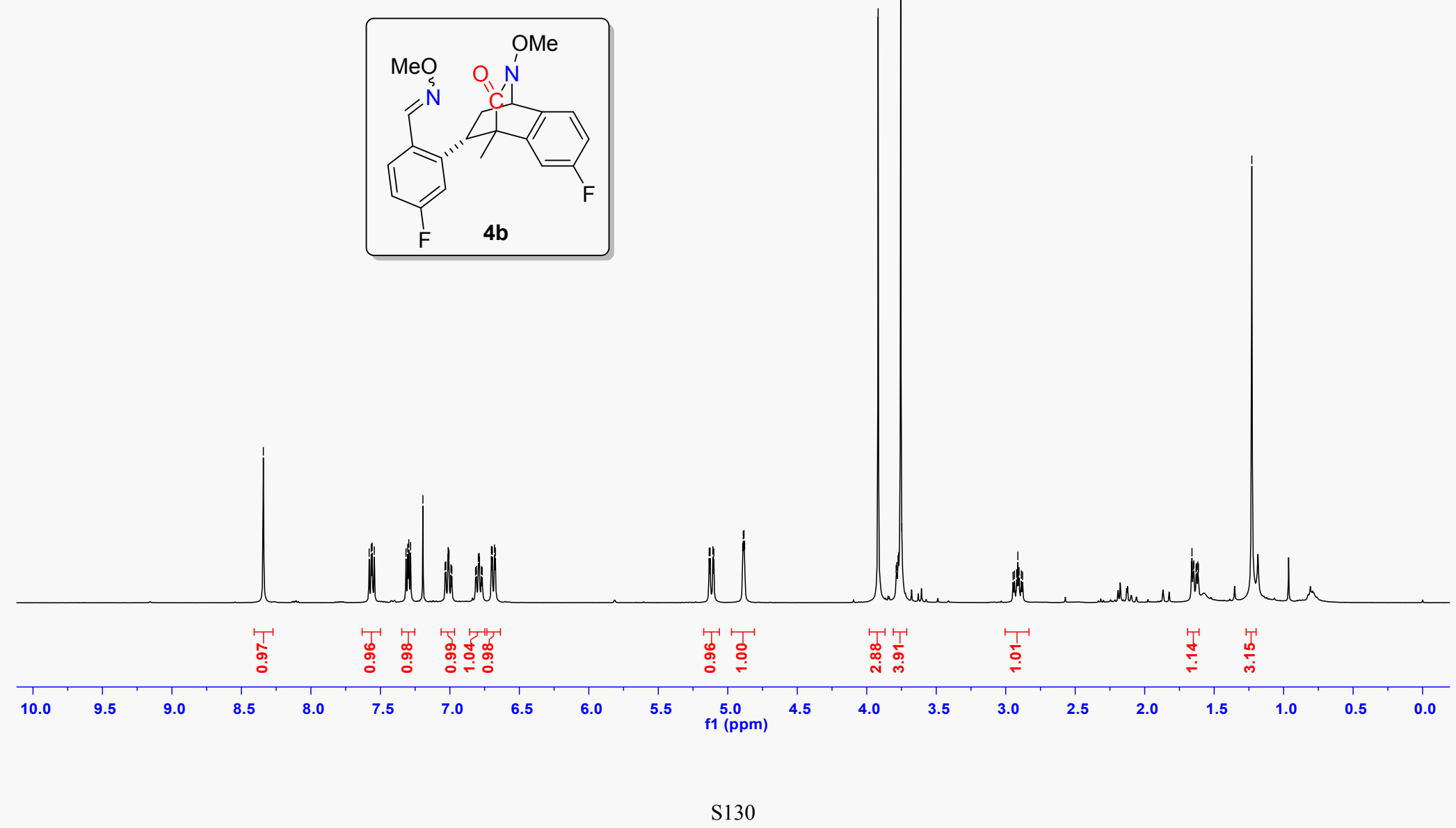


4b-C (100 MHz, CDC13)
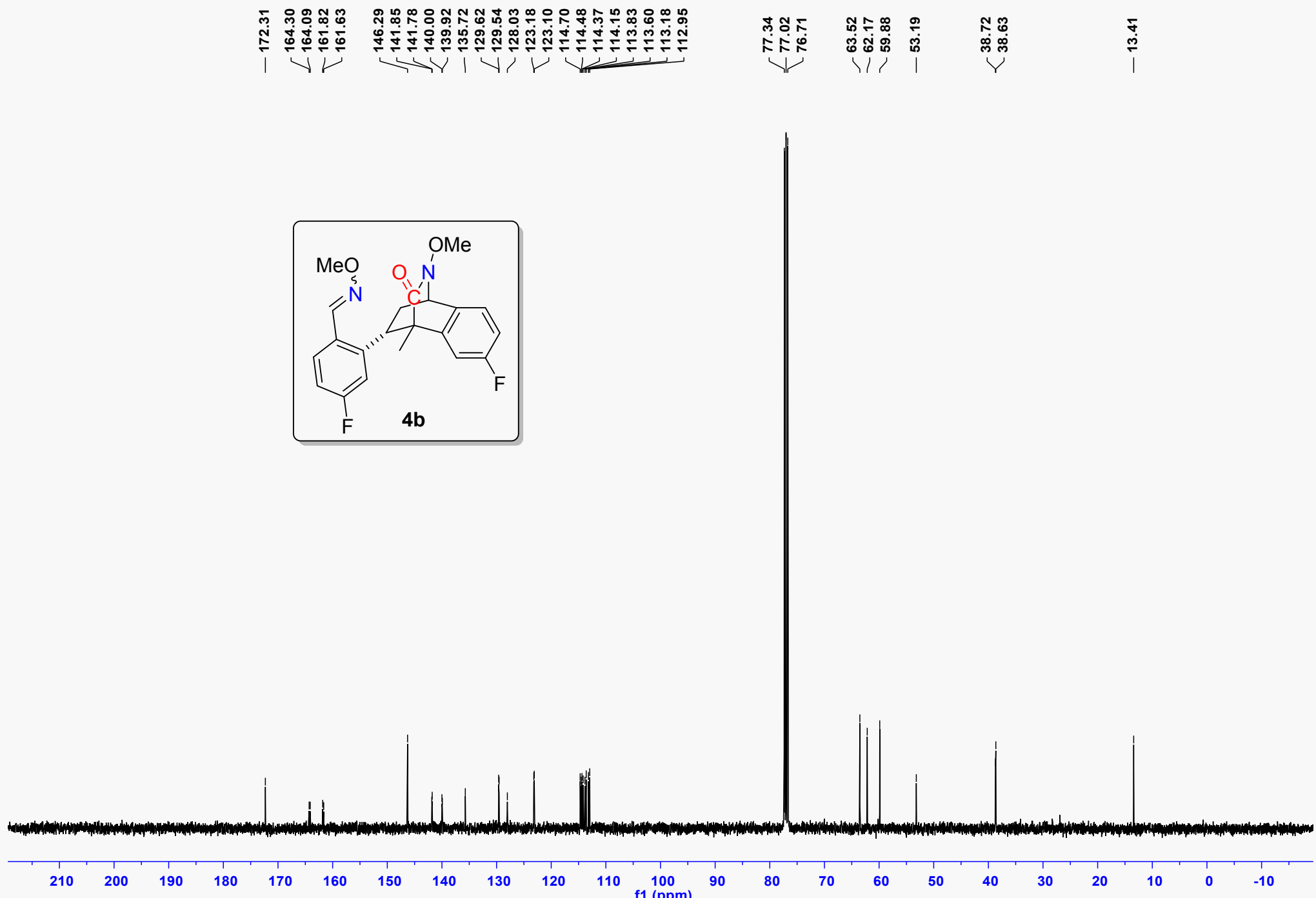
4b-F $(376 \mathrm{MHz}, \quad \mathrm{CDC} 13)$
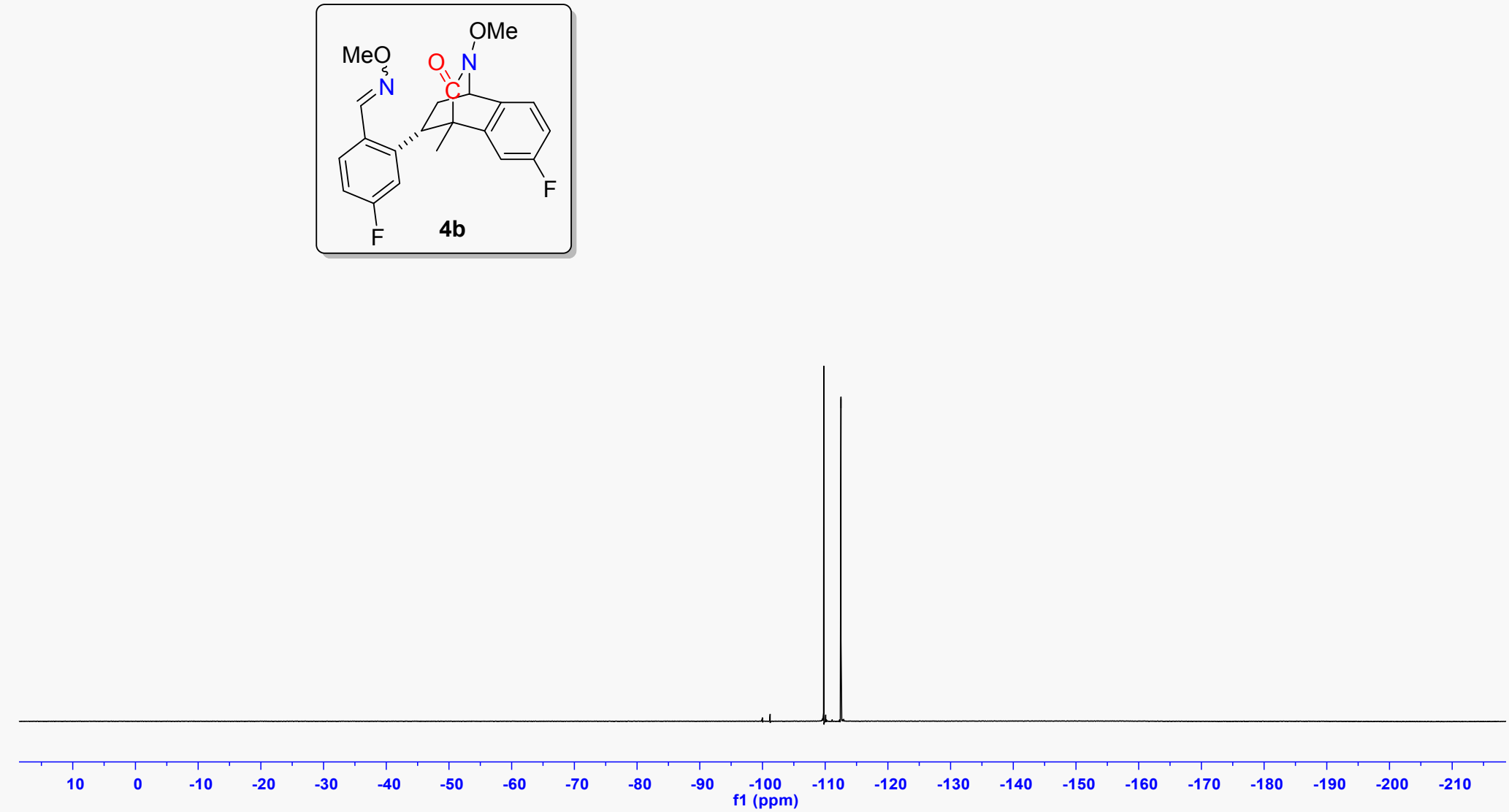


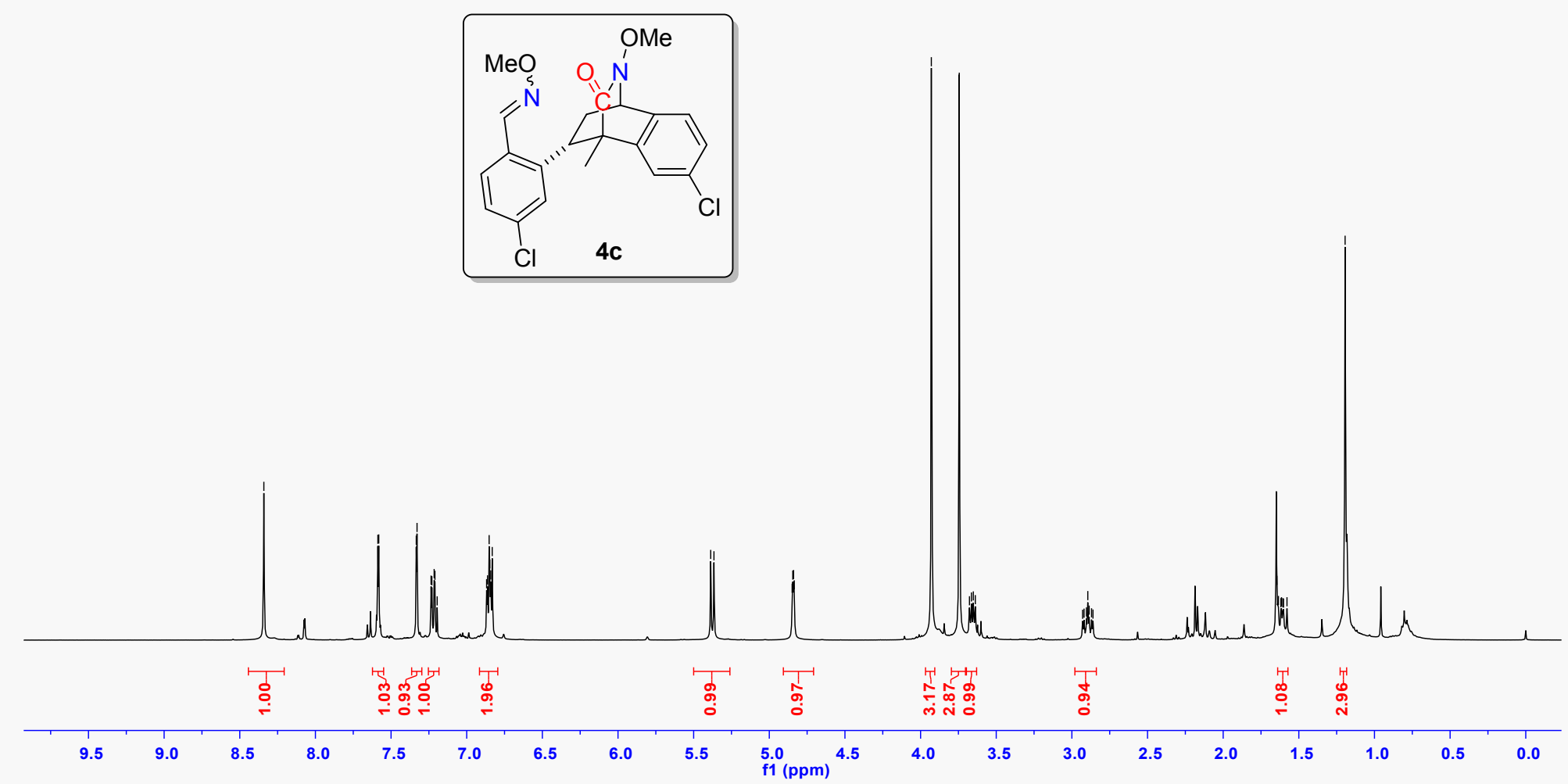


$4 \mathrm{c}-\mathrm{C}(100 \mathrm{MHz}, \mathrm{CDC13})$

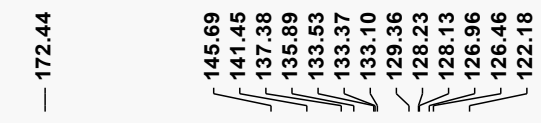

年

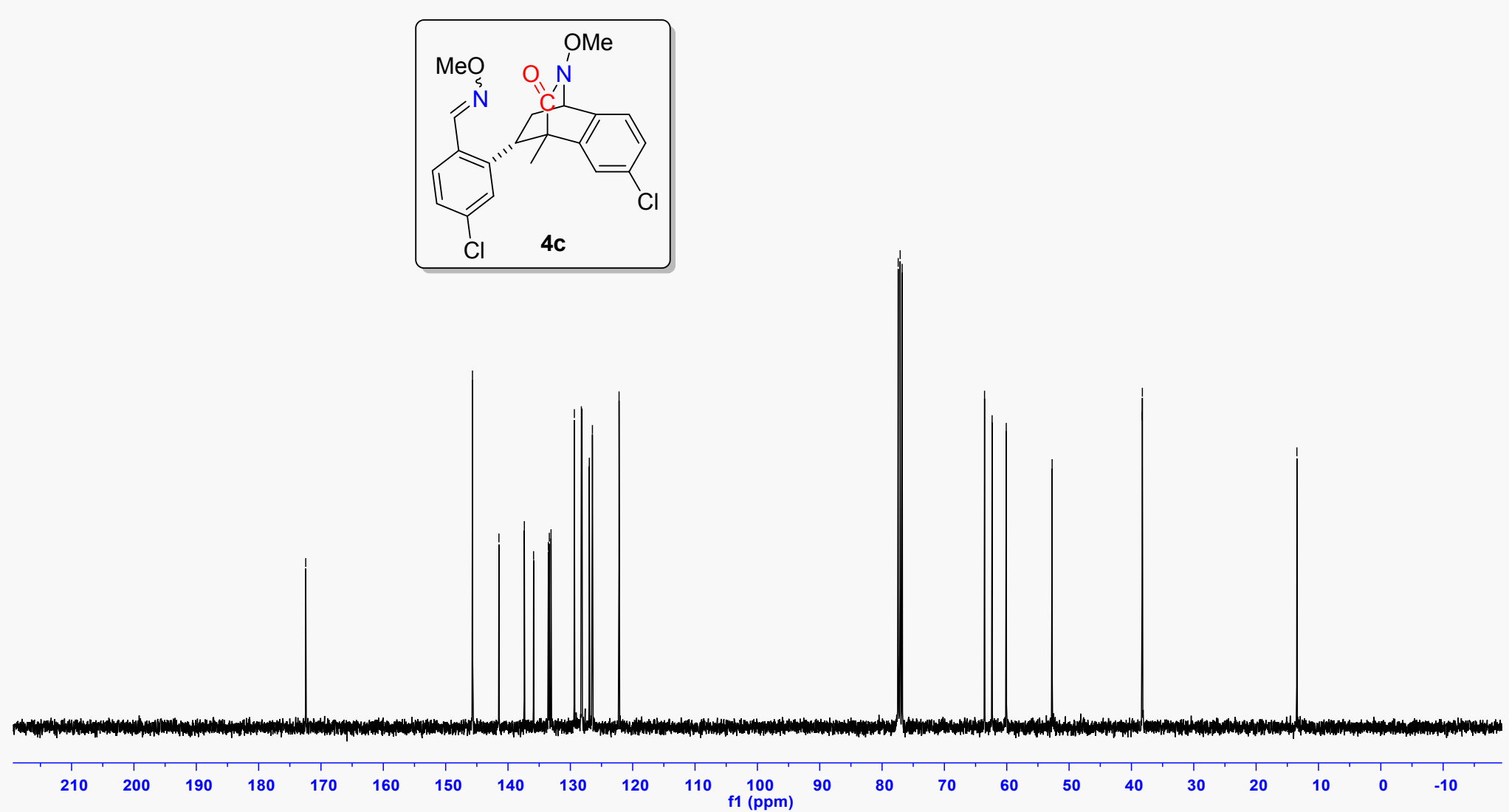


$4 \mathrm{~d}-\mathrm{H}(400 \mathrm{MHz}, \mathrm{CDC} 13)$

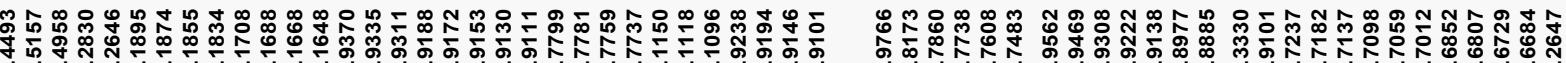

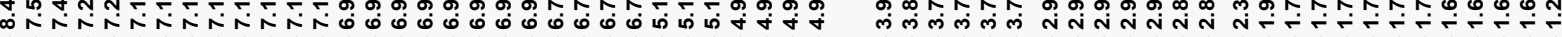

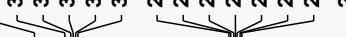

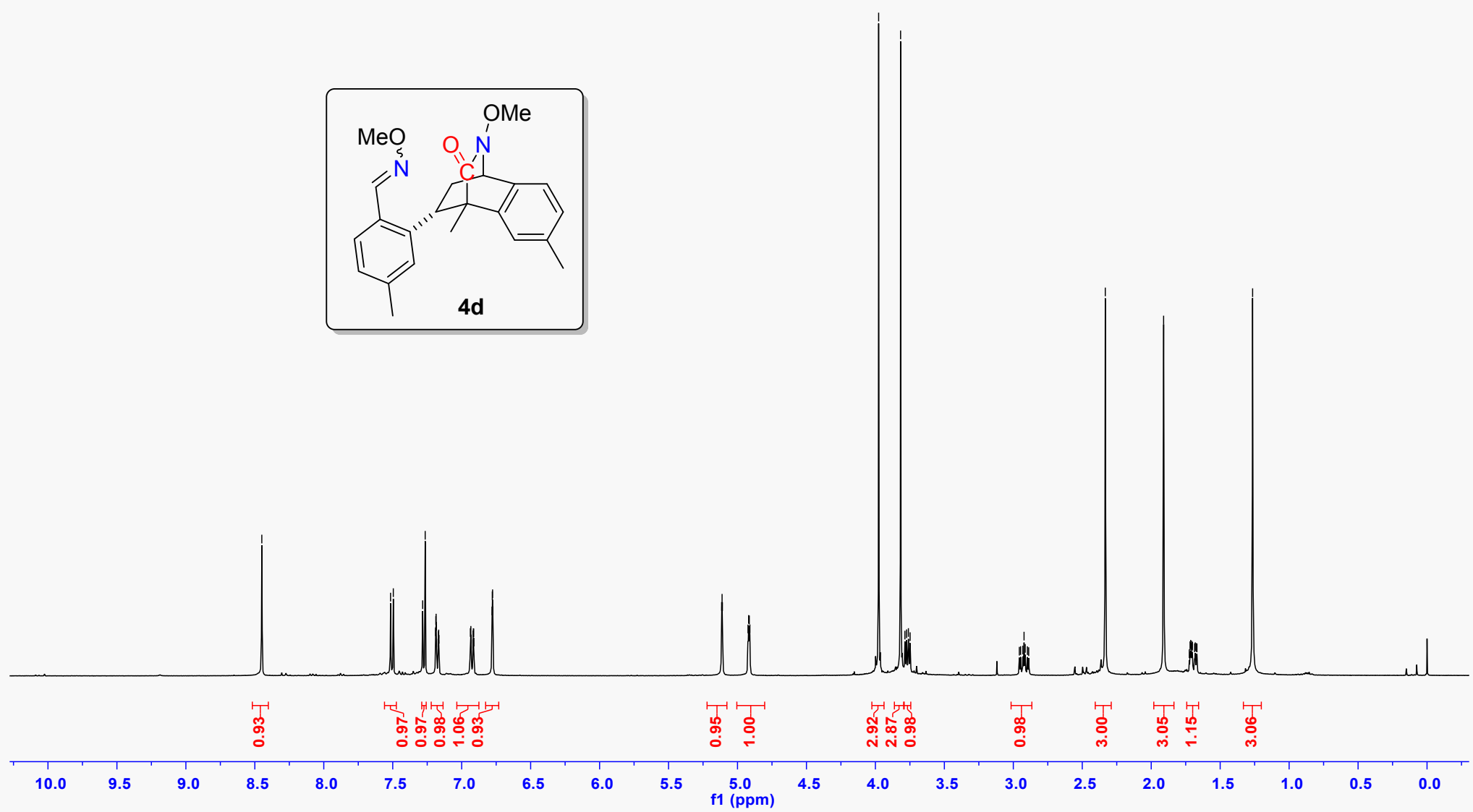


4d-C (100 MHz, CDC13)

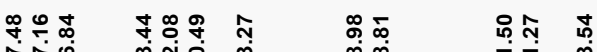

l

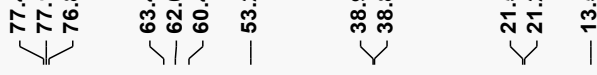
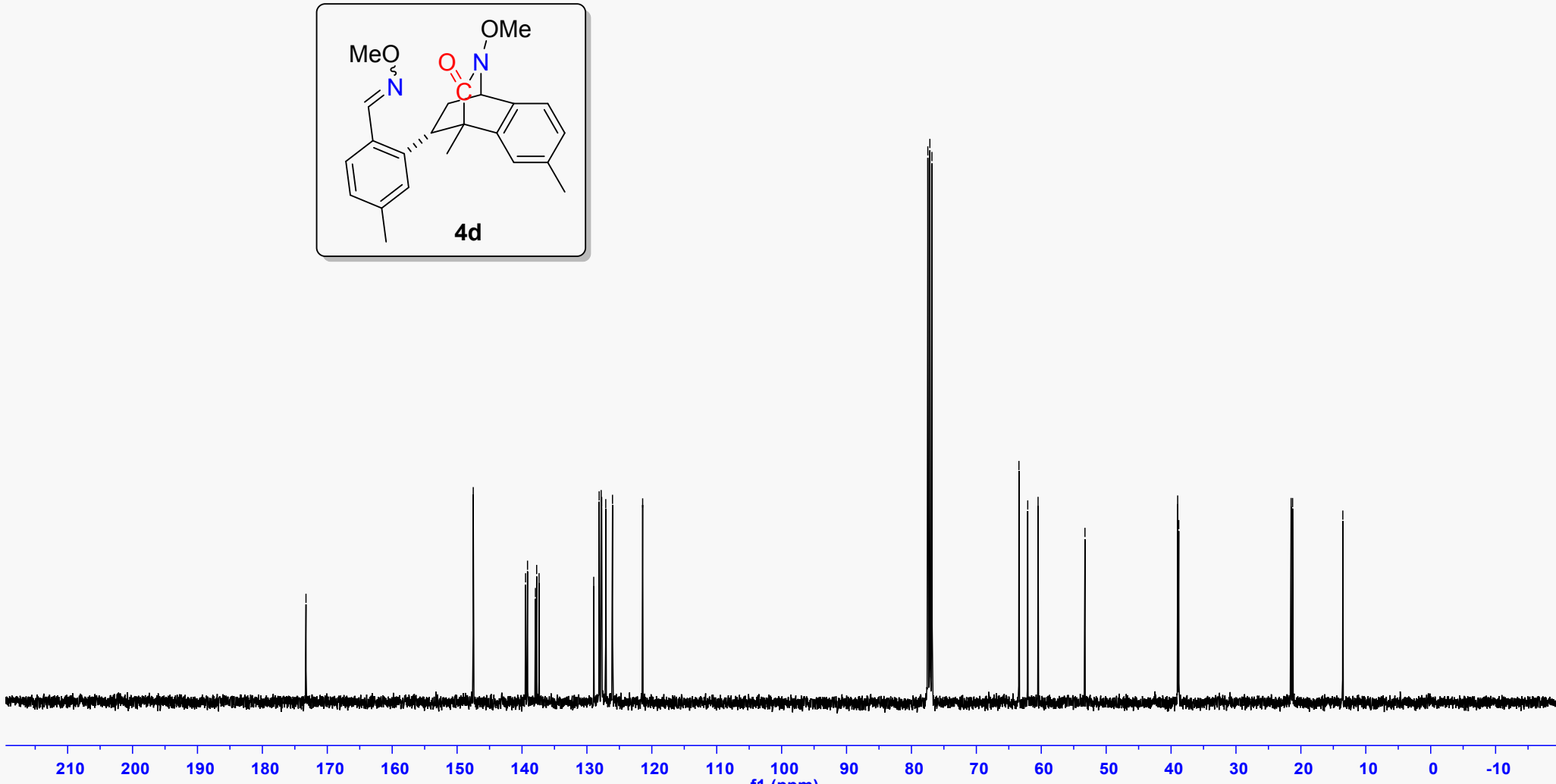
4e-H (400 MHz, CDC13)

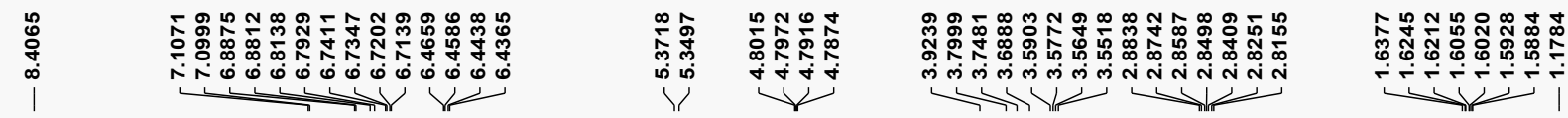

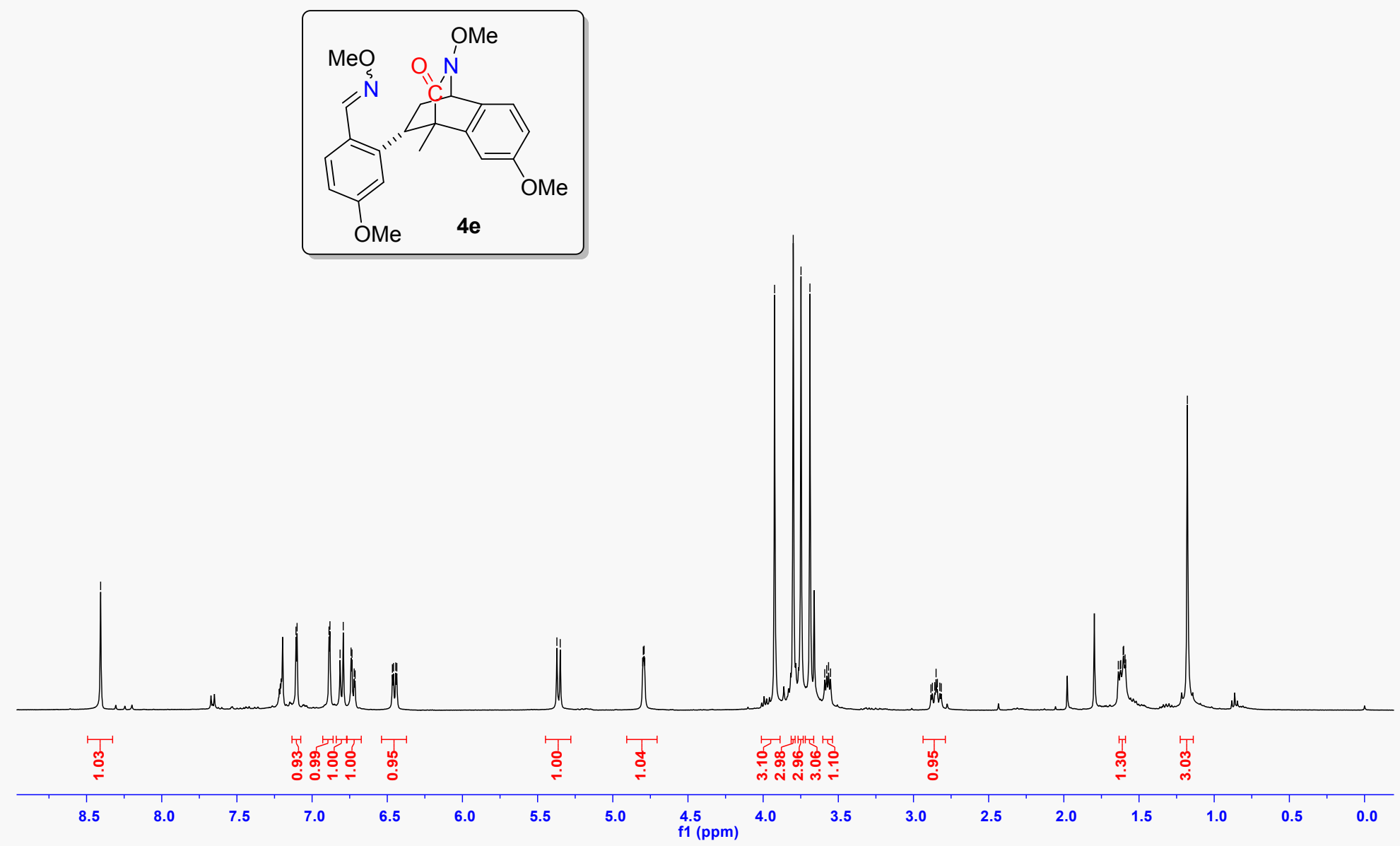



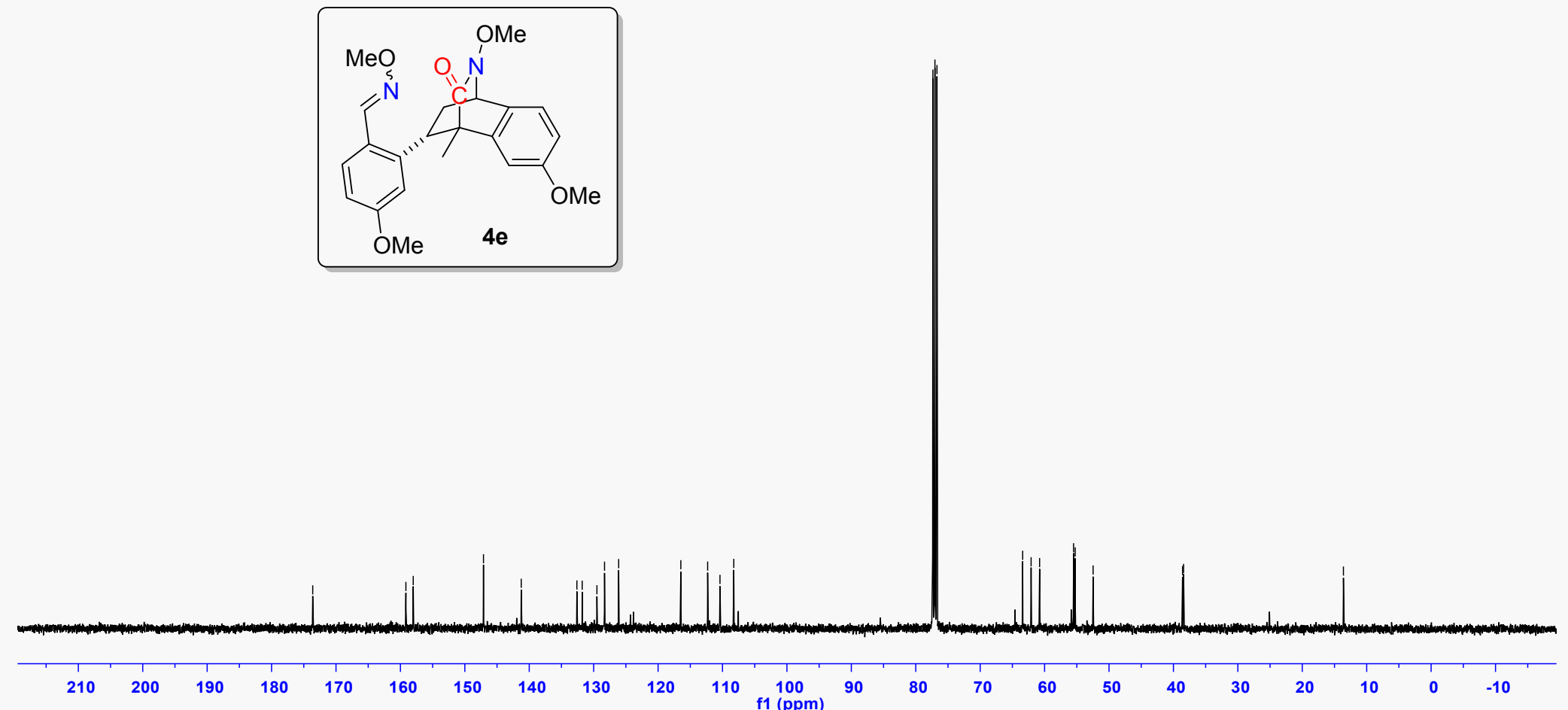
4f-H $(400 \mathrm{MHz}, \quad \mathrm{CDC13})$

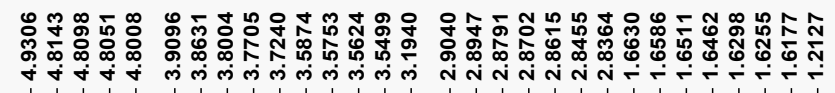

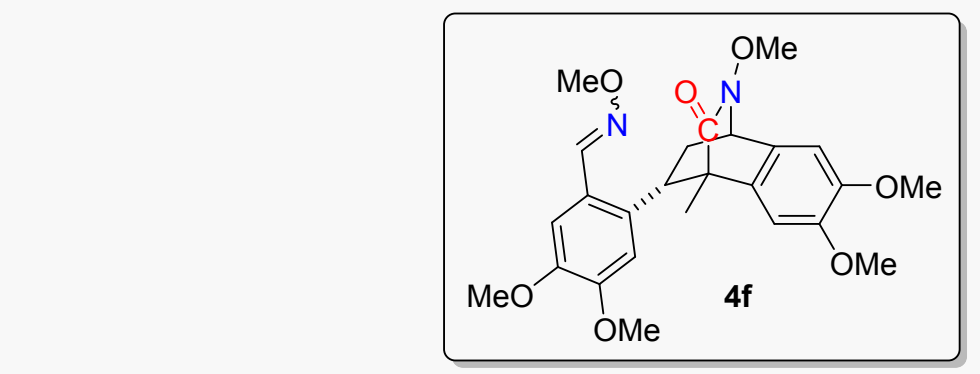

+ं广
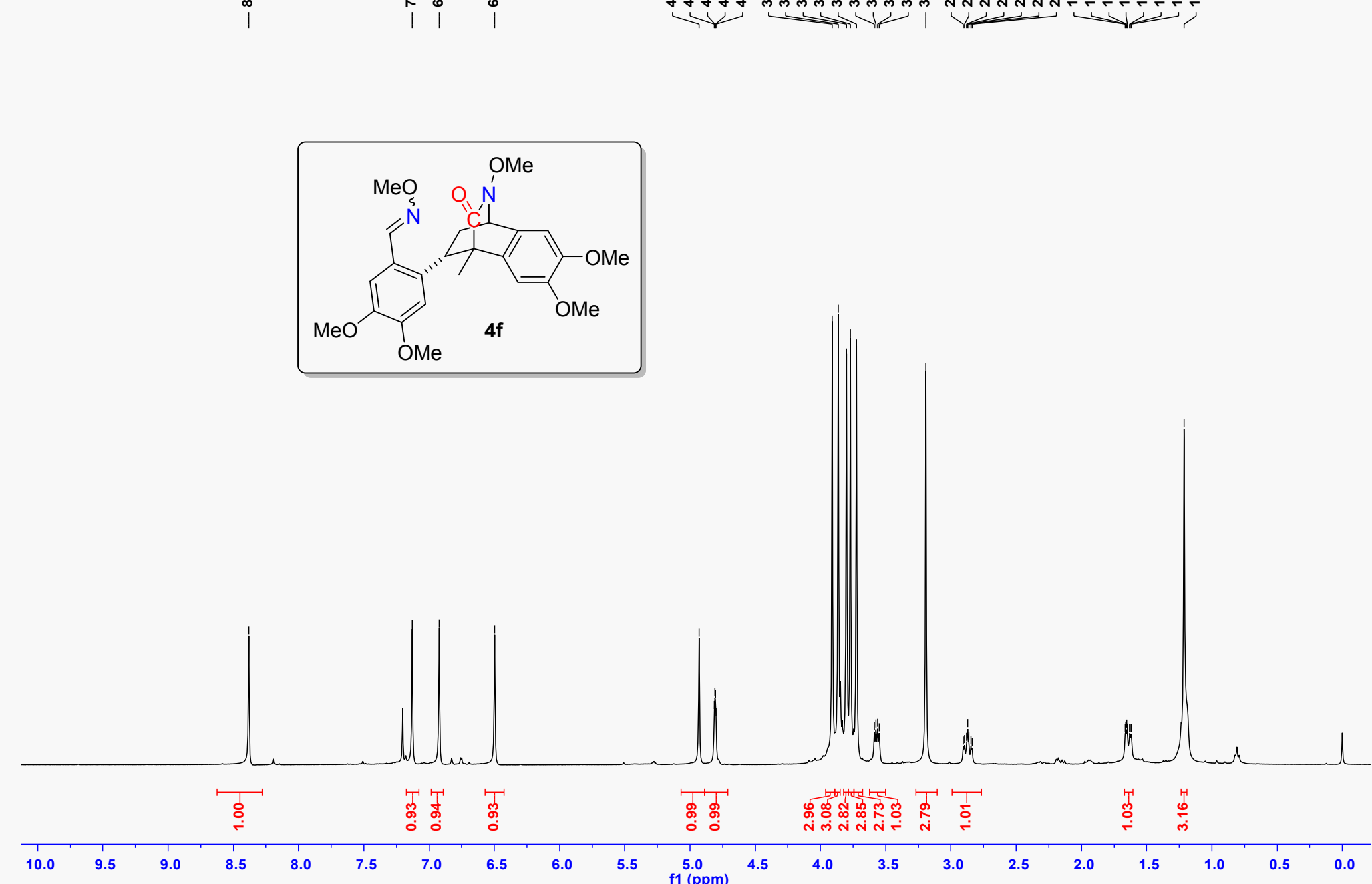


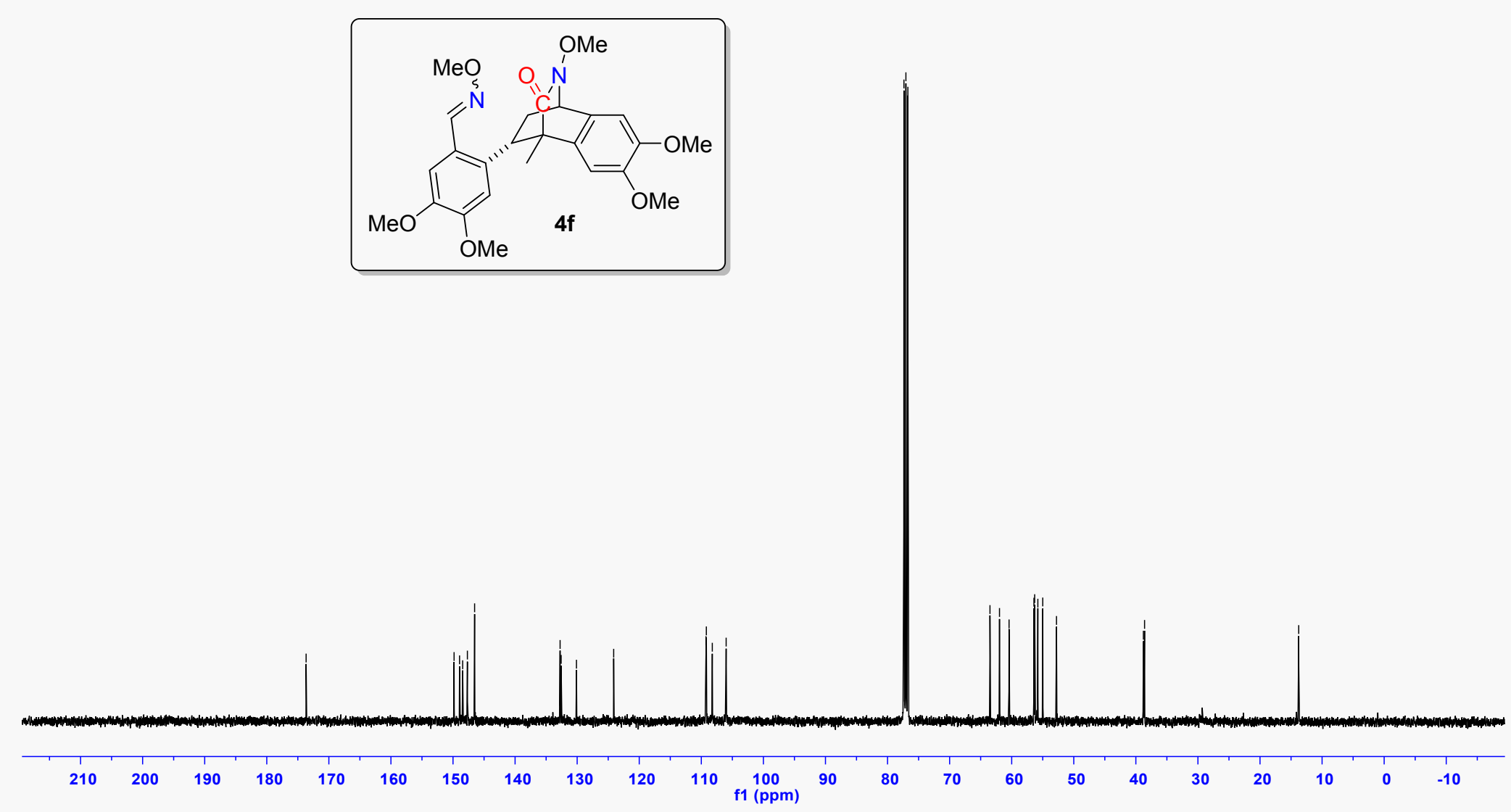


$4 \mathrm{~g}-\mathrm{H}(400 \mathrm{MHz}, \mathrm{CDCl} 3)$

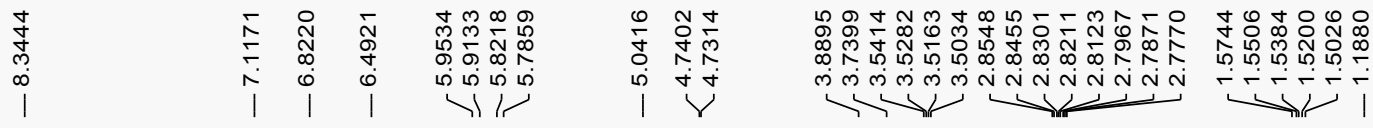
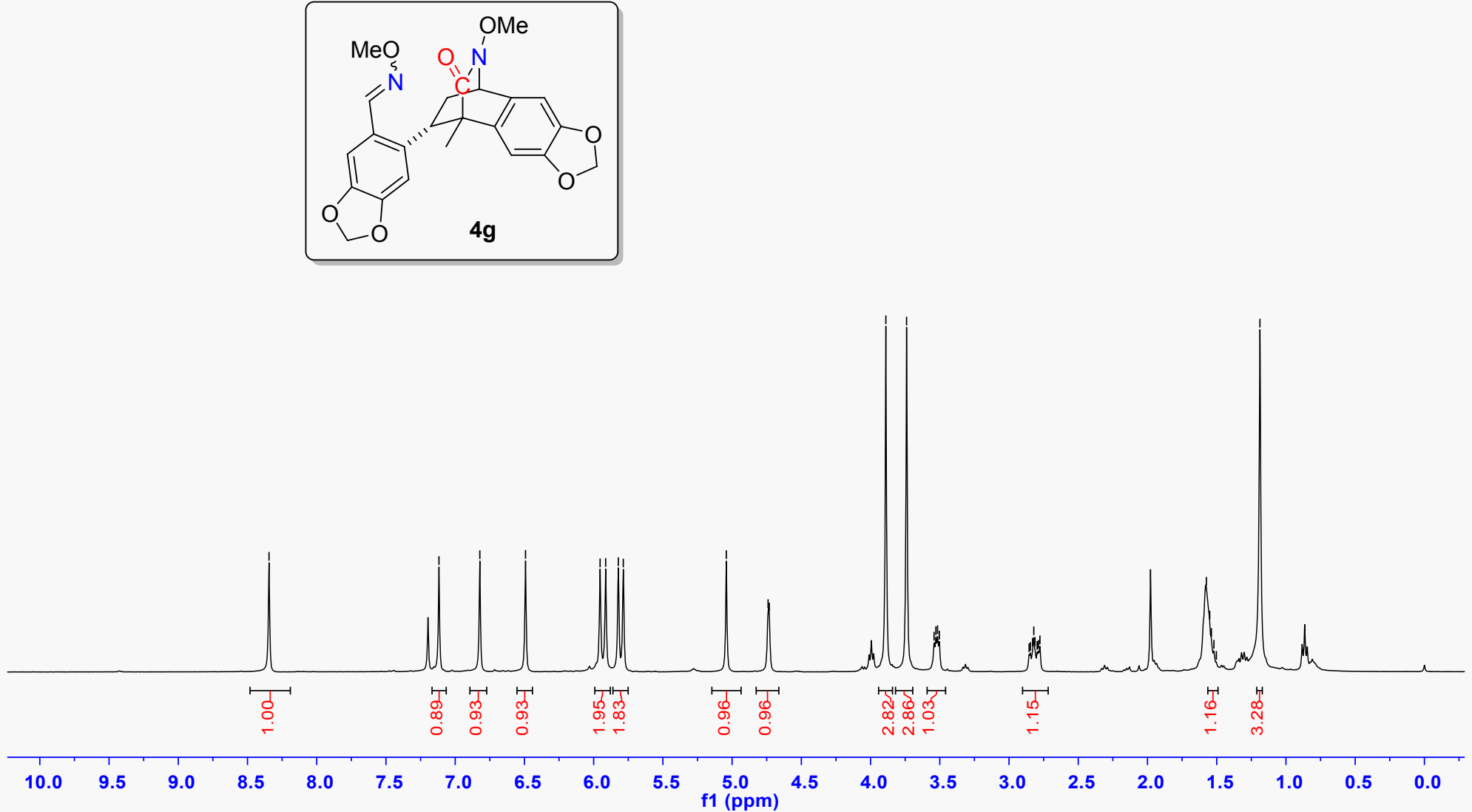
$4 \mathrm{~g}-\mathrm{C}(100 \mathrm{MHz}, \mathrm{CDCl} 3)$

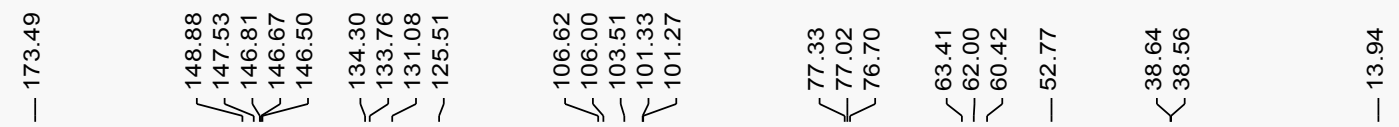

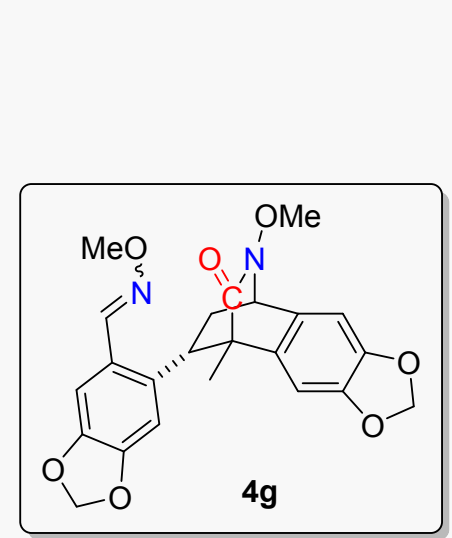

000 \%
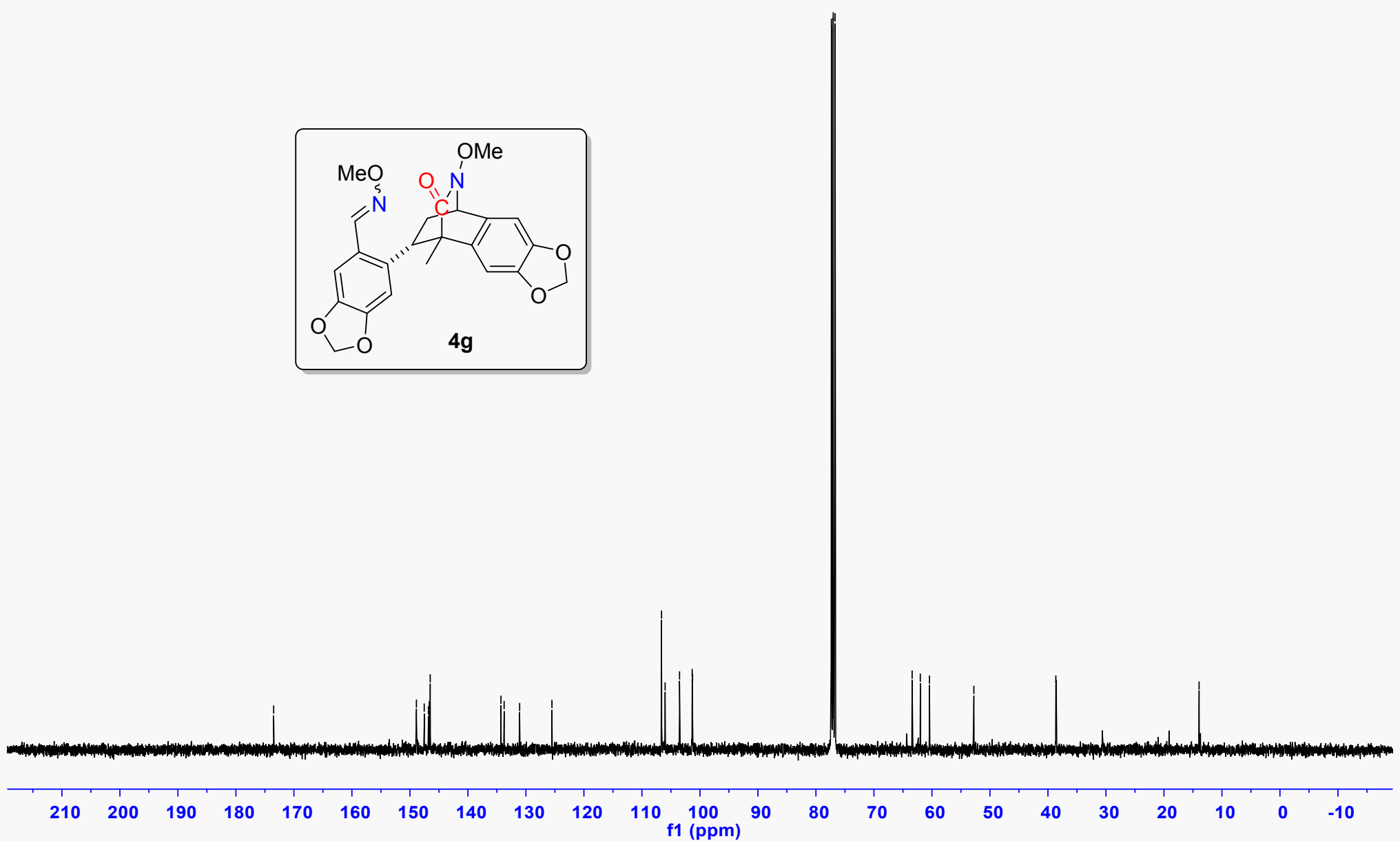

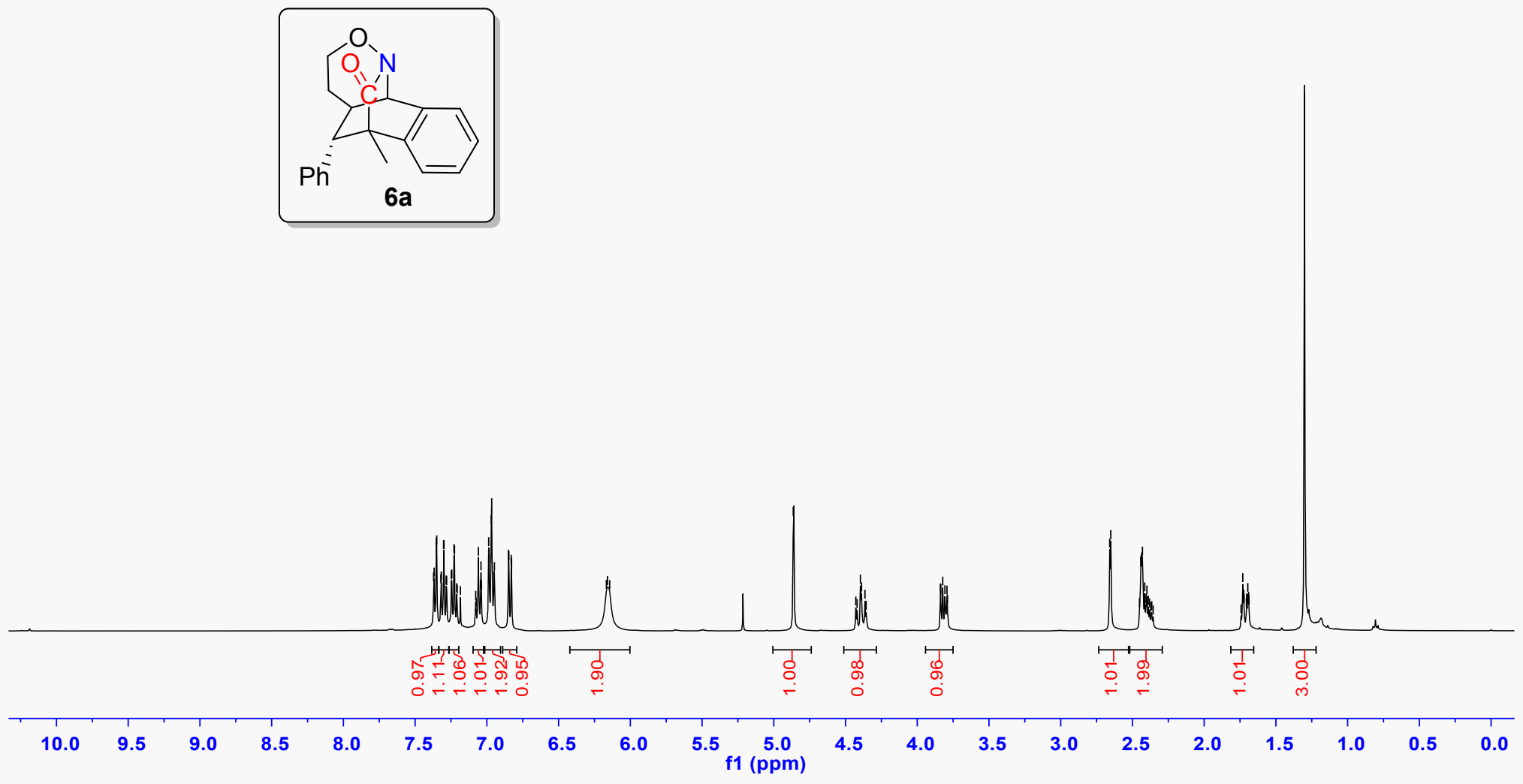
6a-C (100 MHz, CDCI3)

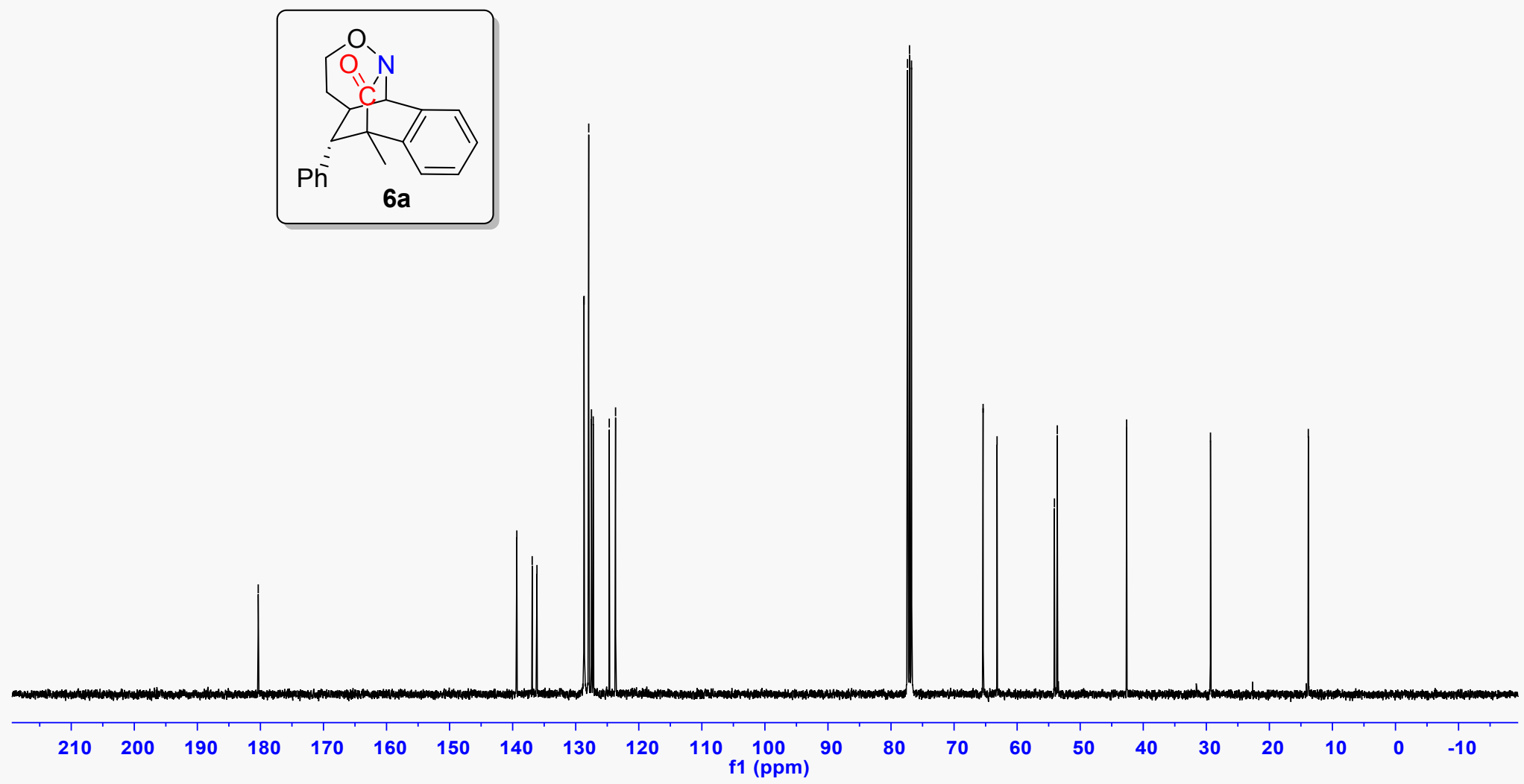

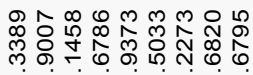

。ํำ

\प

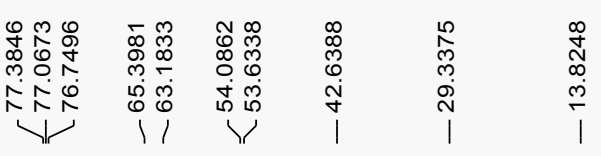



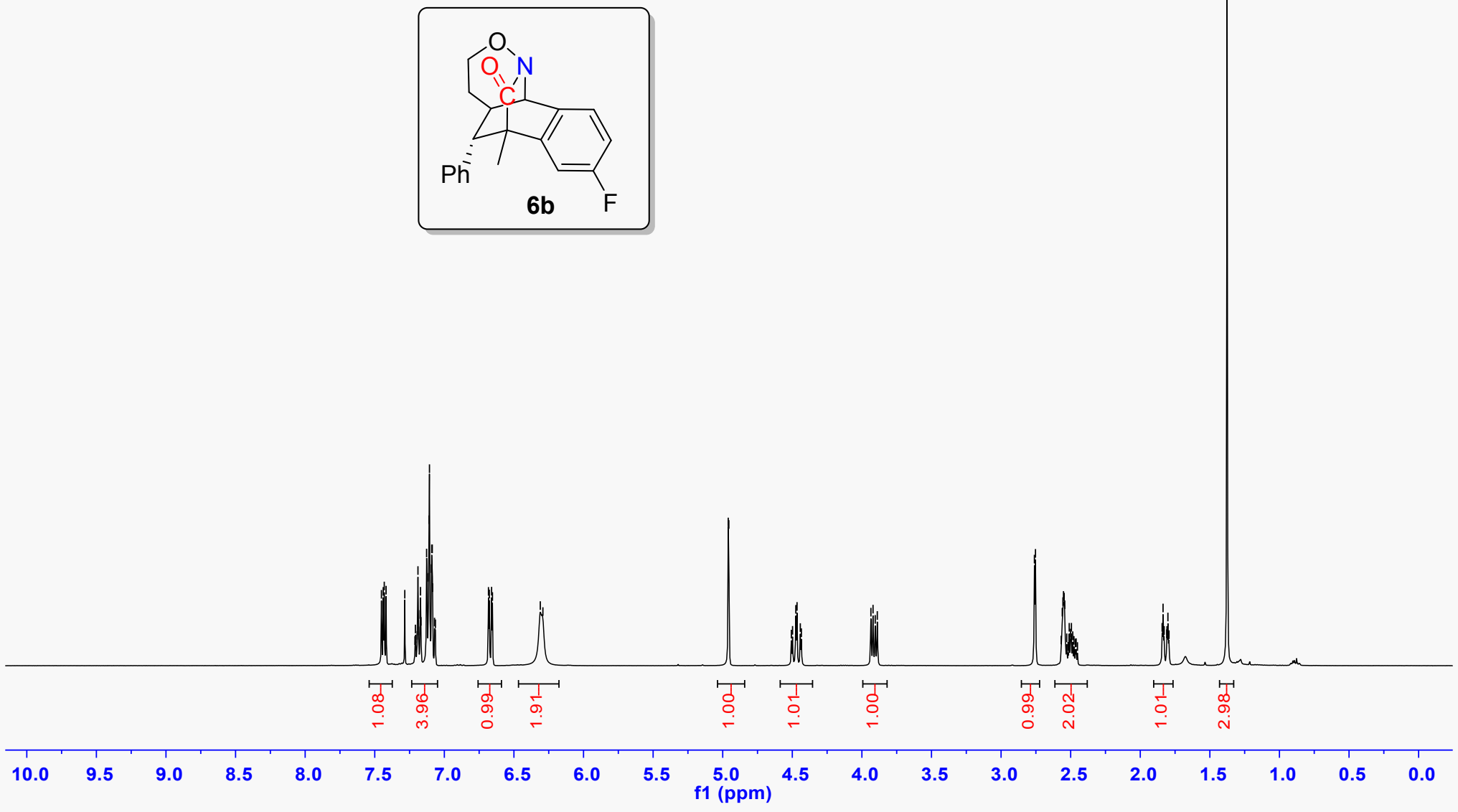
6b-C (100 MHz, CDCI3)

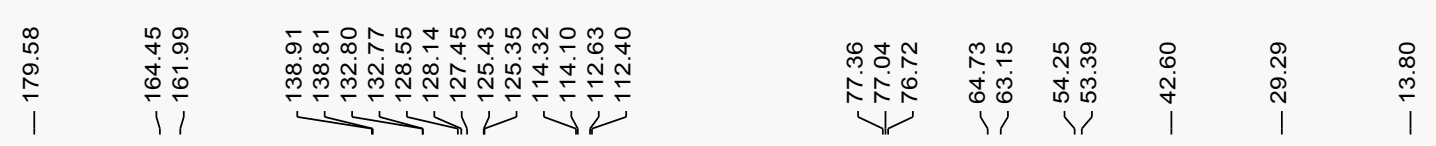

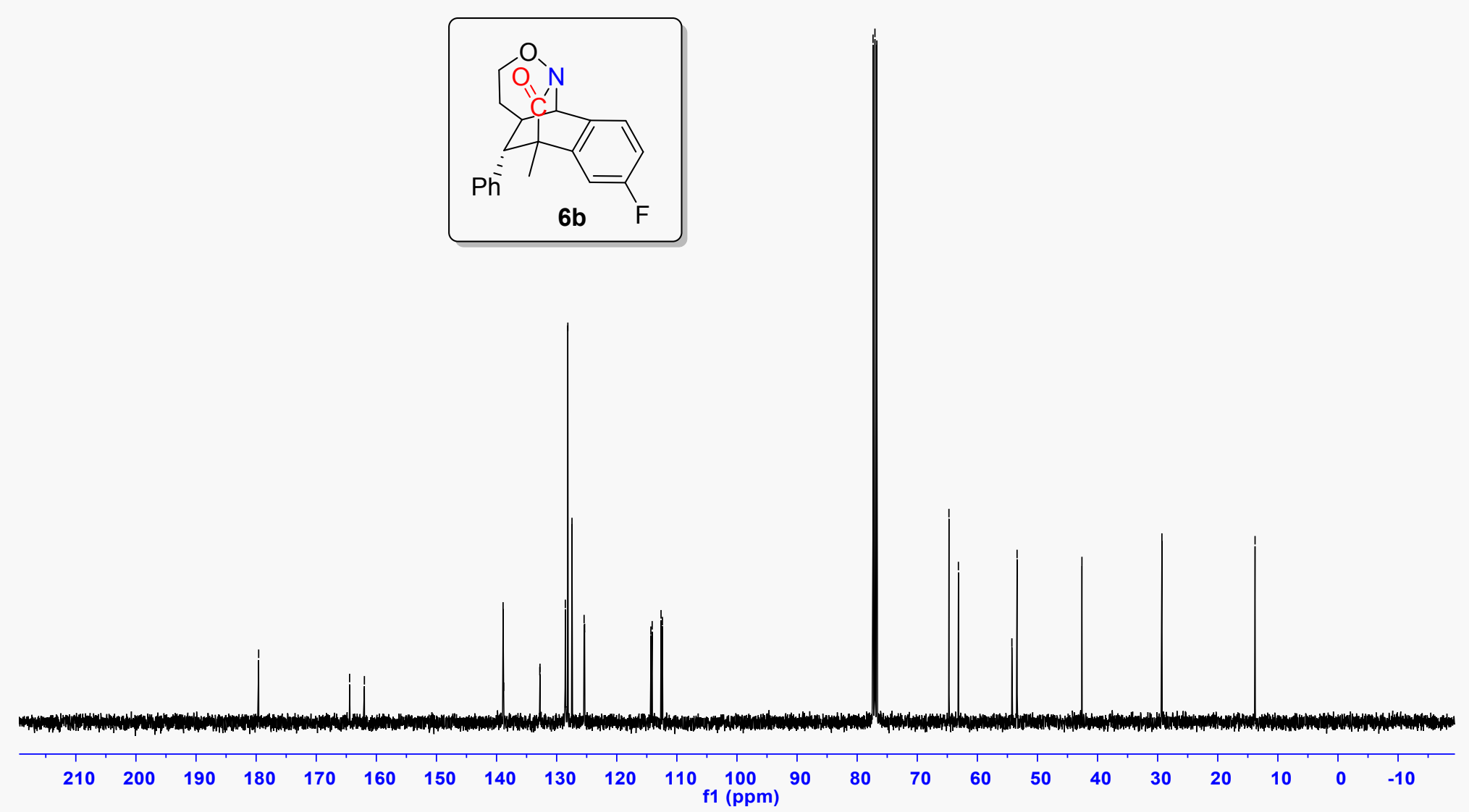


6b-F (376 MHz, CDCI3)

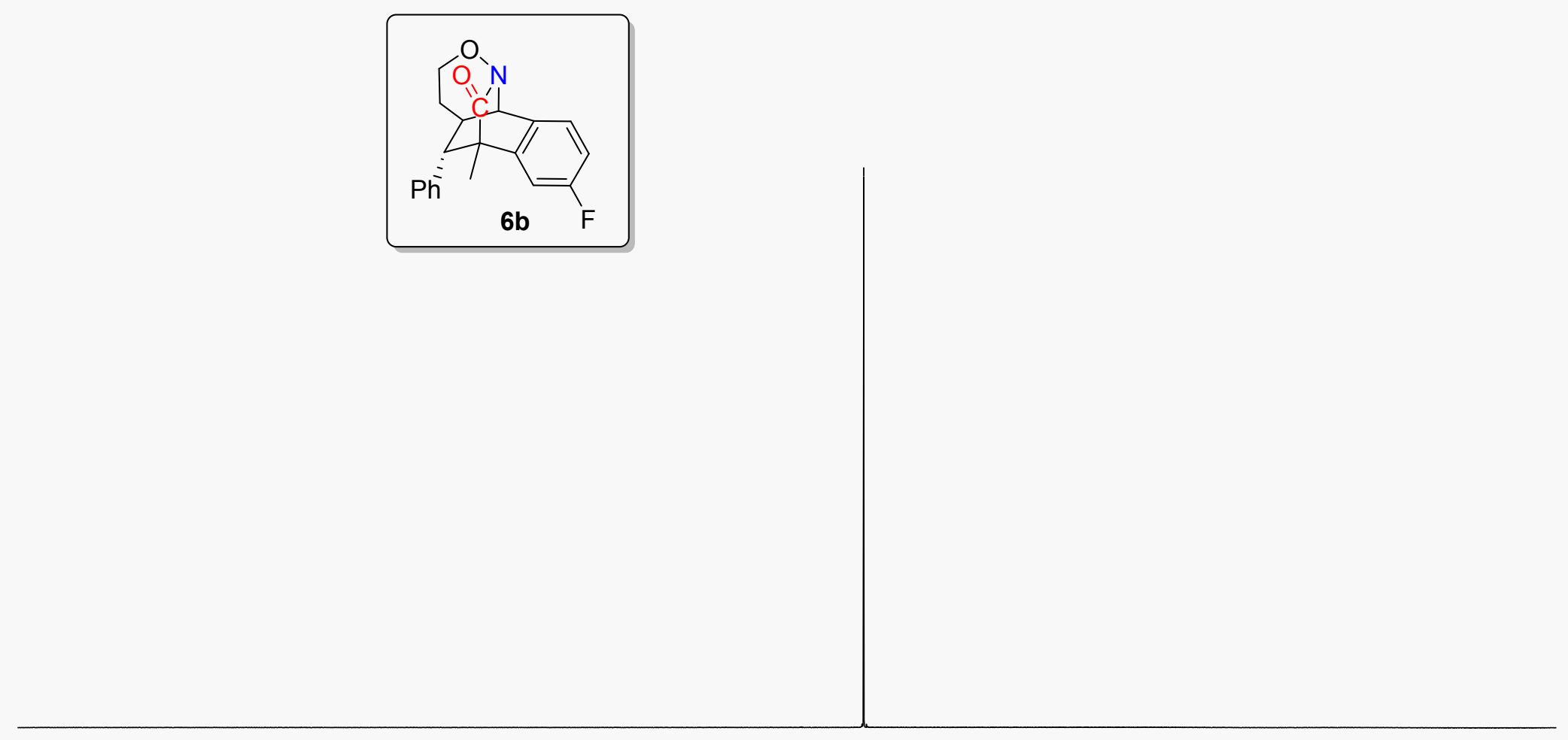


$6 \mathrm{c}-\mathrm{H}(400 \mathrm{MHz}, \mathrm{CDCl} 3)$

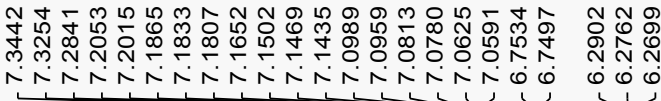

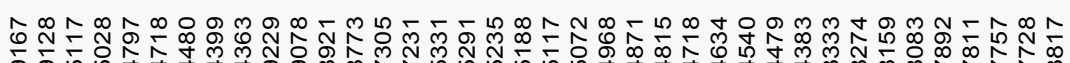

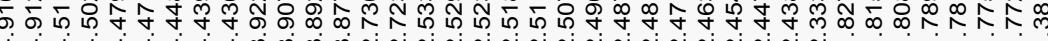

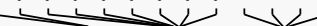
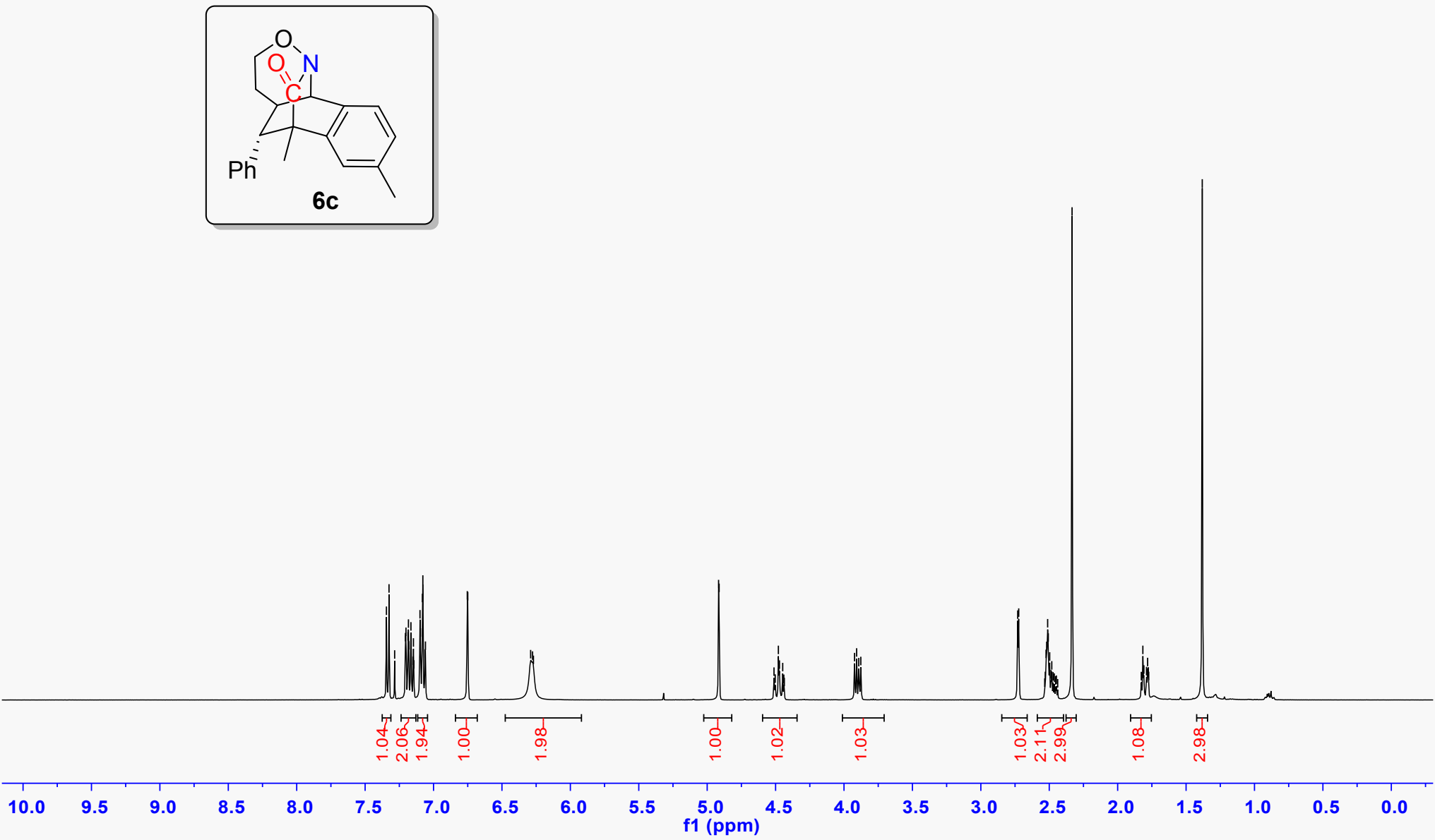
6c-C (100 MHz, CDCl3)

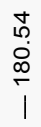

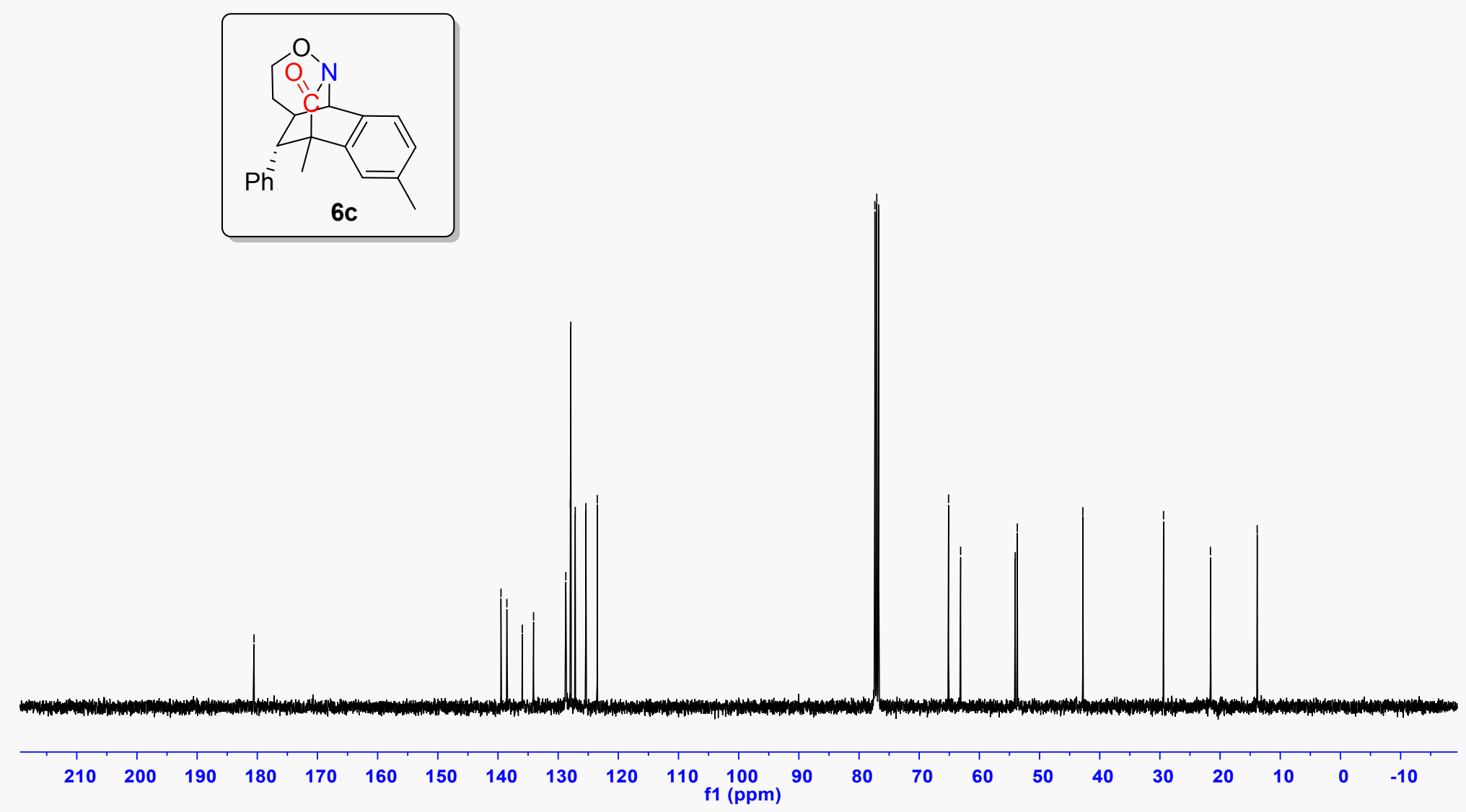

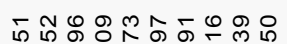

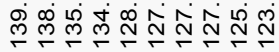

$\underbrace{2}$ 
$6 \mathrm{~d}-\mathrm{H}(400 \mathrm{MHz}, \mathrm{CDCl} 3)$

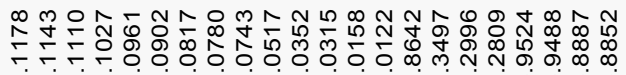

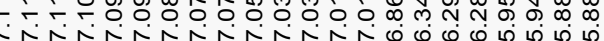

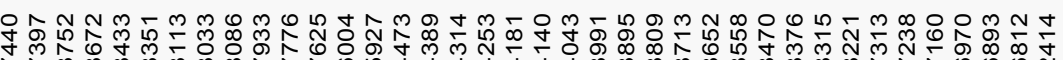

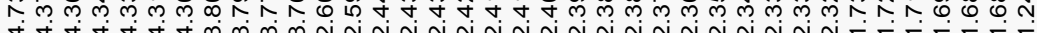

等
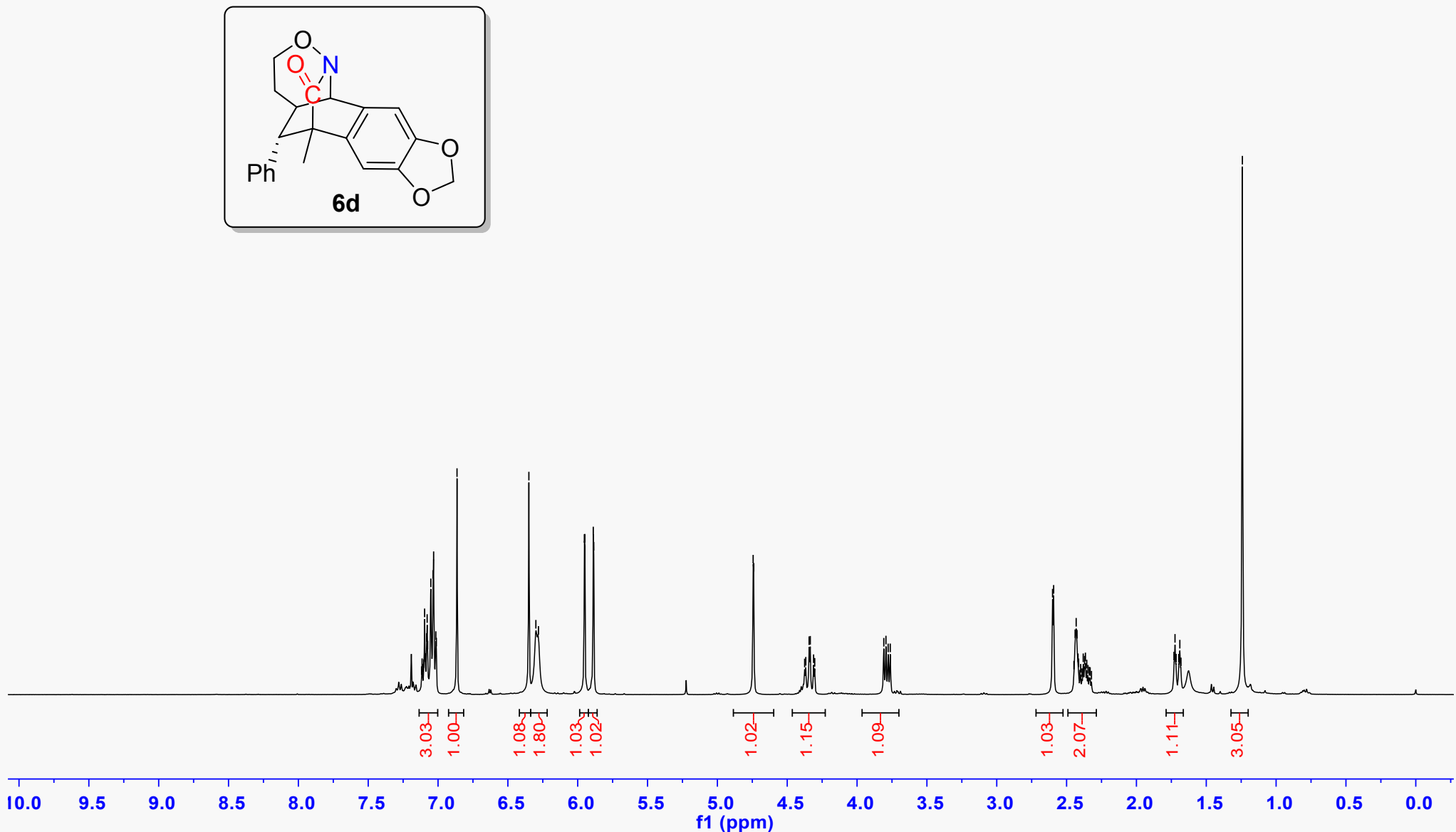
6d-C (100 MHz, CDCl3)

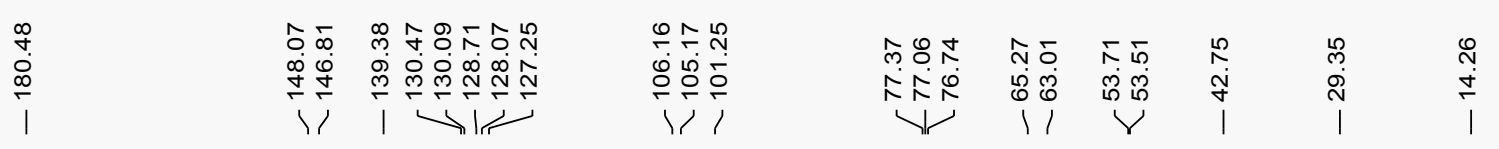
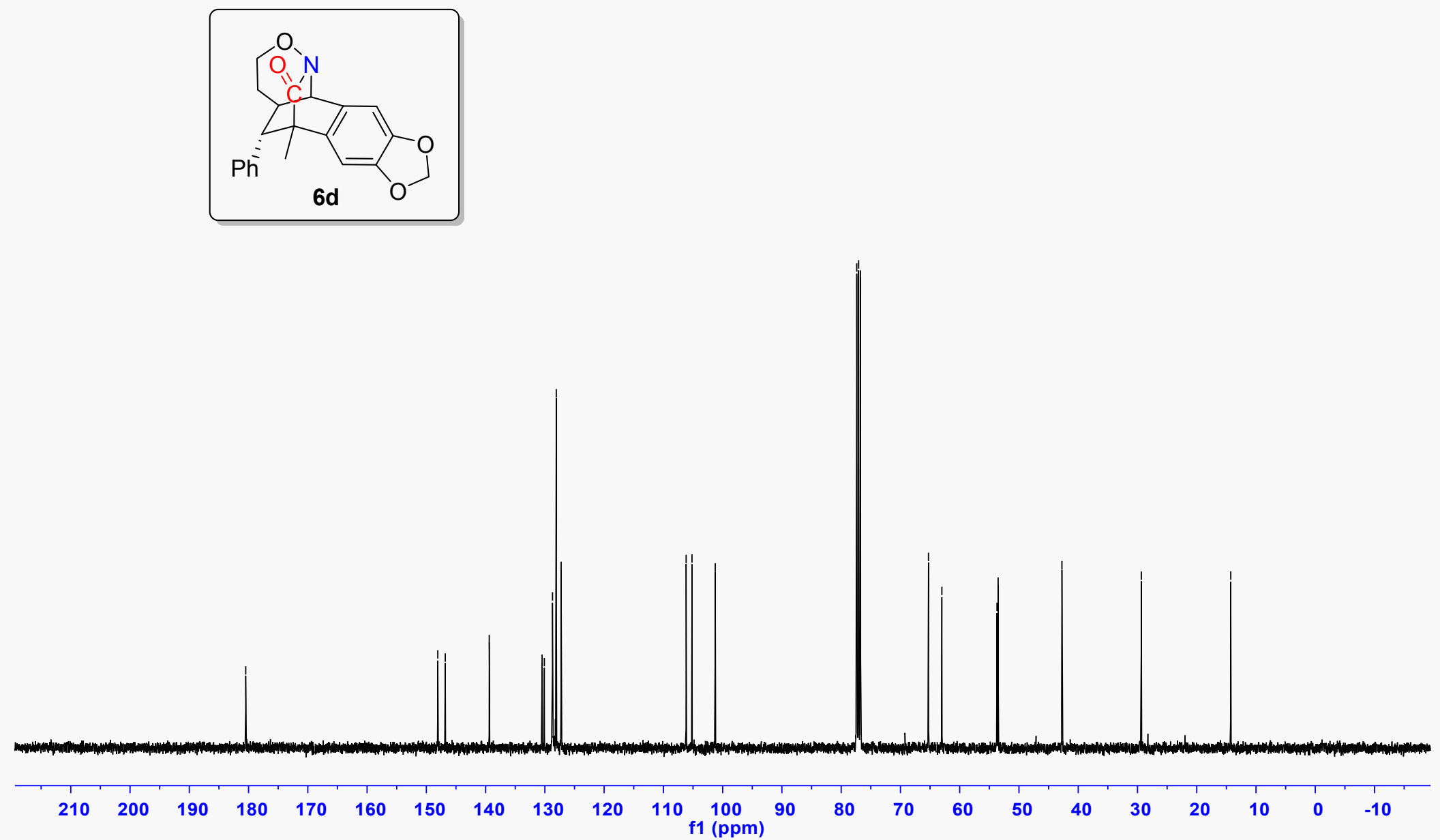


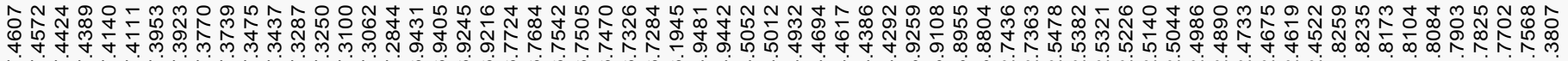
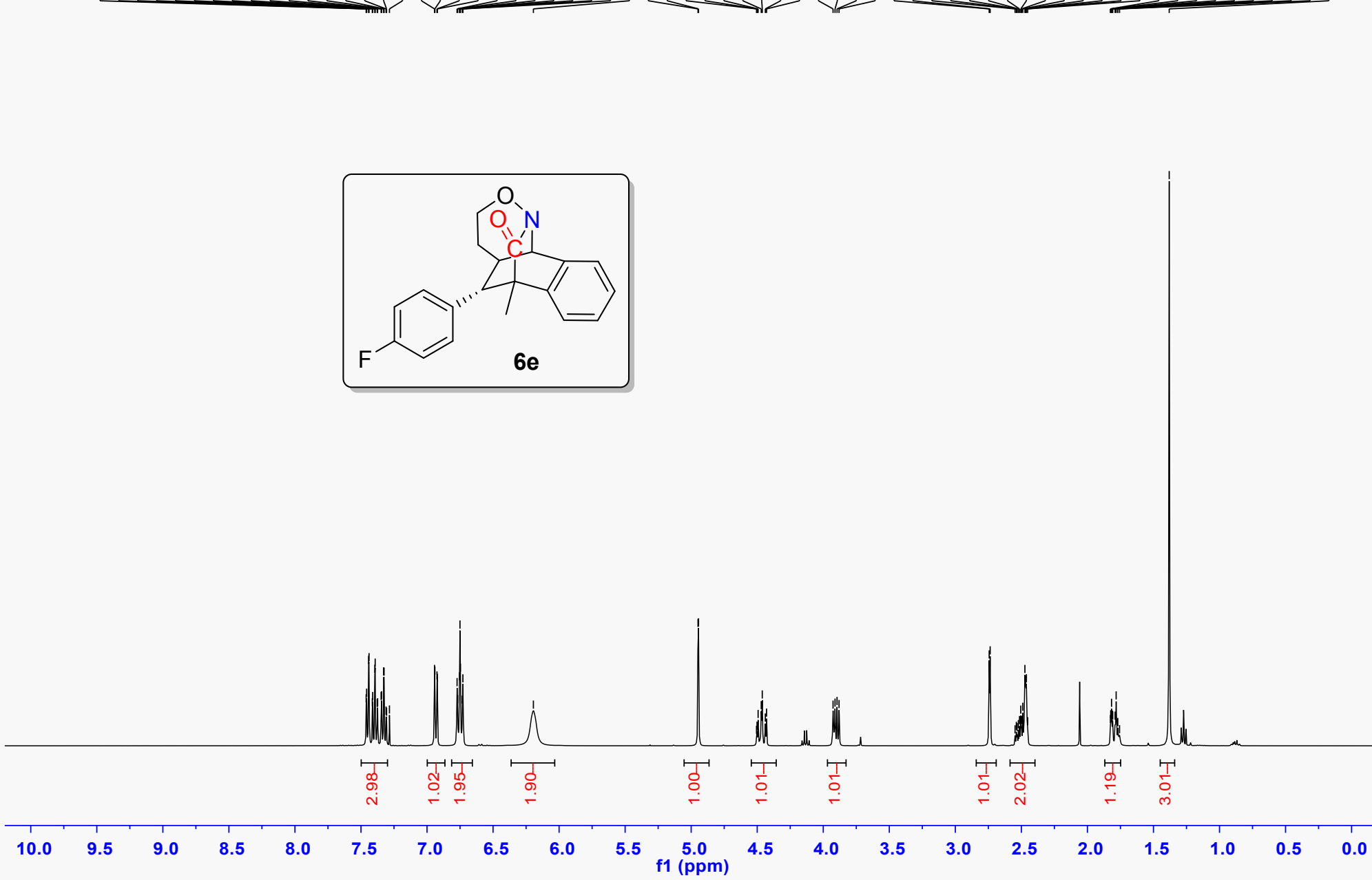
6e-C (100 MHz, CDCl3)

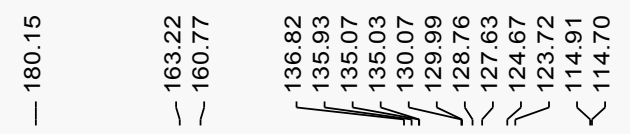

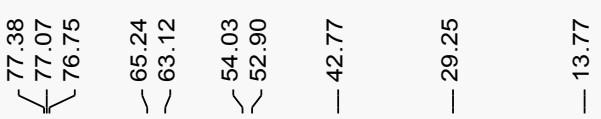
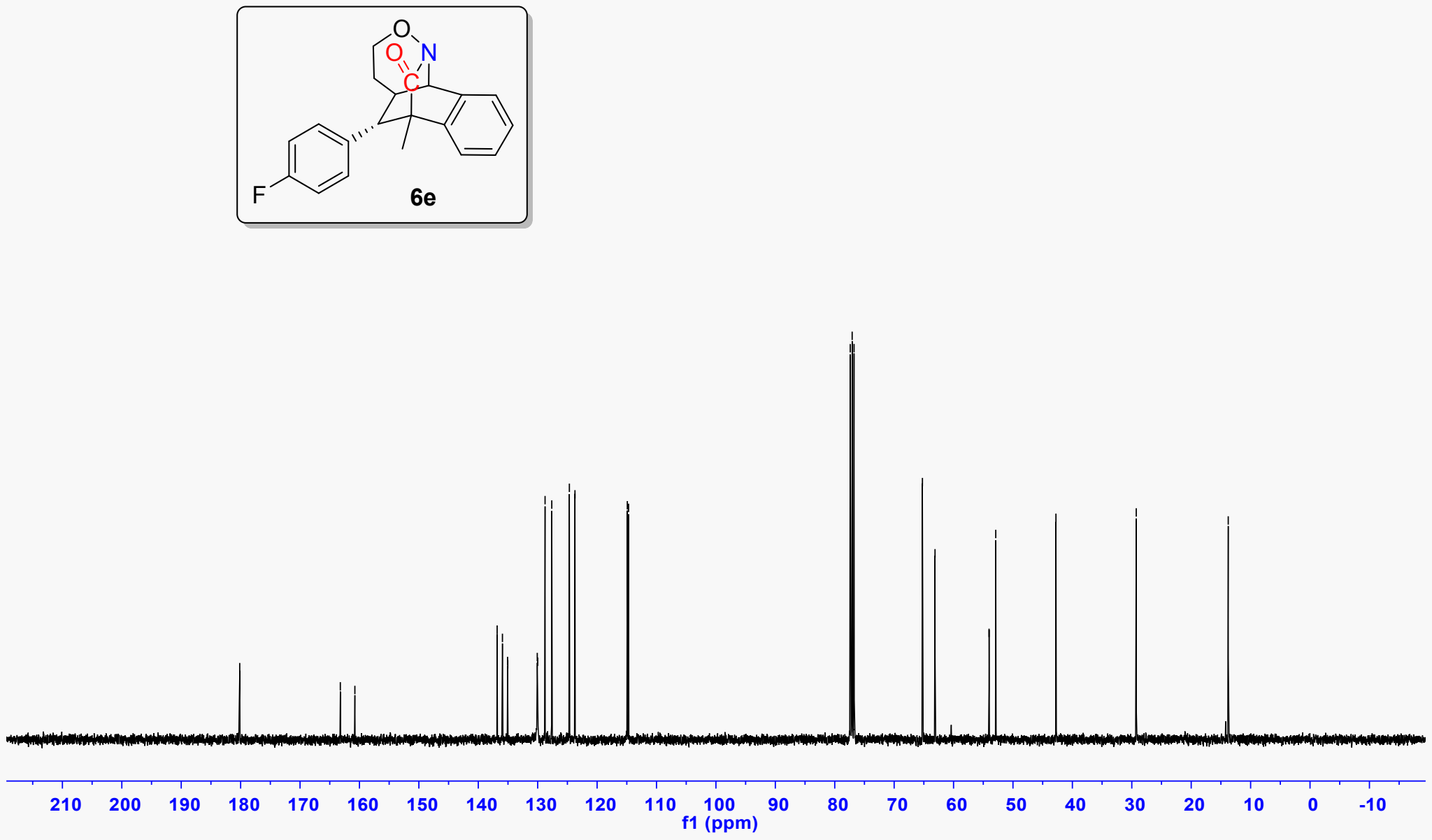
6e-F(376 MHz, CDCl3)

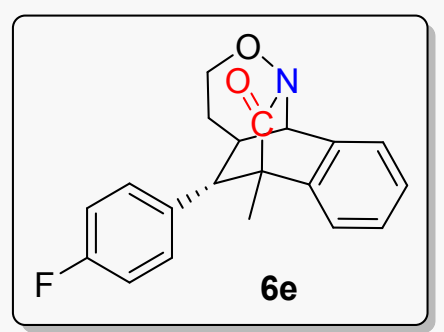

$10 \quad 0 \quad-10$

$\begin{array}{llllllllllll}-100 & -110 & -120 & -130 & -140 & -150 & -160 & -170 & -180 & -190 & -200 & -210\end{array}$ 
6f-H (400 MHz, CDCl3)

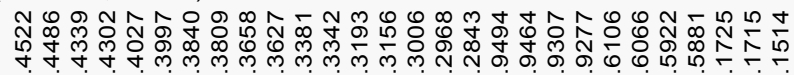

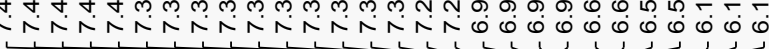

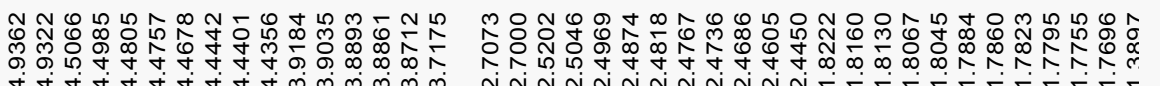

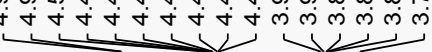

.

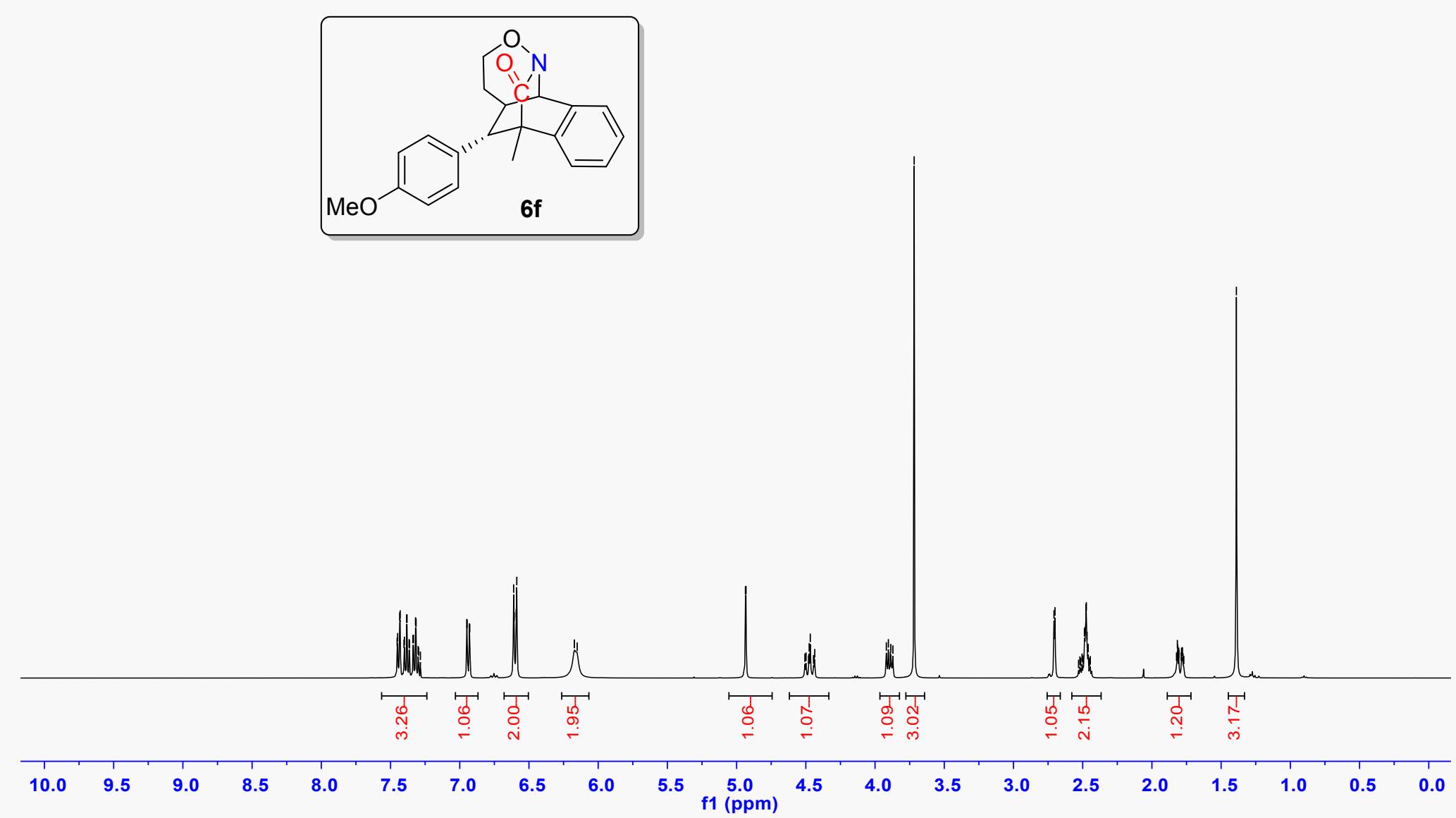


6f-C (100 MHz, CDCl3)

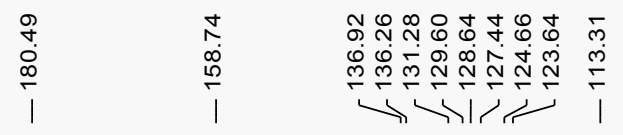

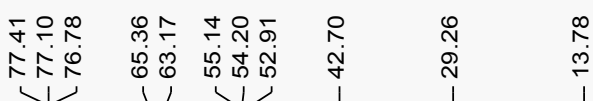
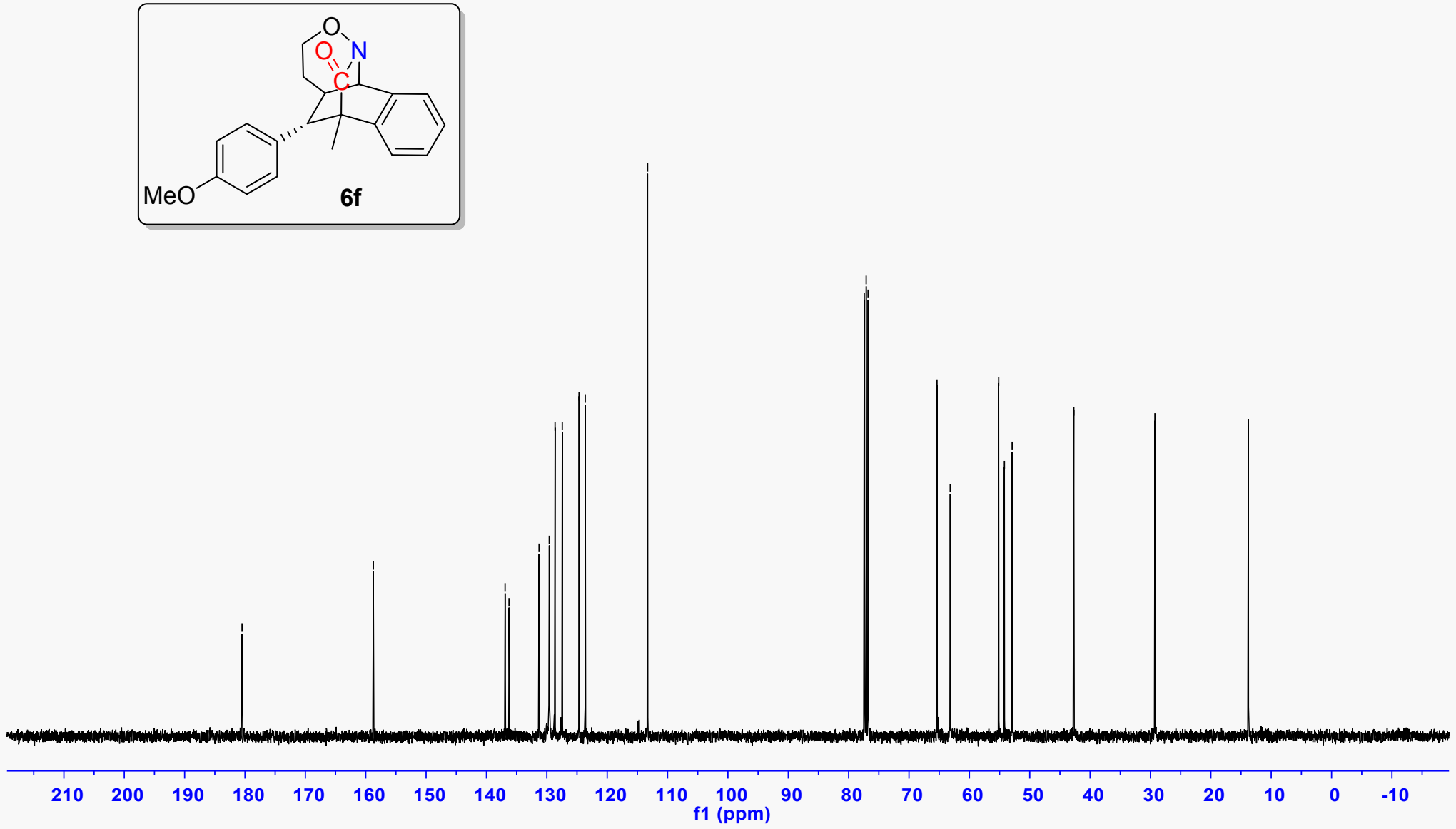